\title{
COMBINE7.1 - A Portable ENDF/B-VII.0 Based Neutron Spectrum and Cross-Section Generation Program
}

W. Y. Yoon

R. A. Grimesey

D. W. Nigg

R. L. Curtis

August 2009

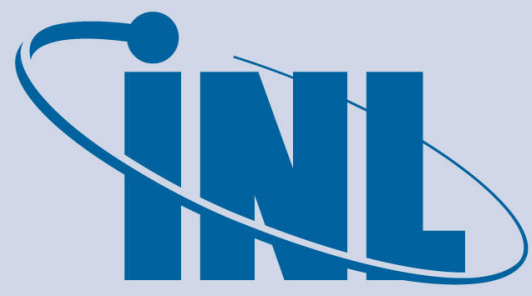

Idaho National Laboratory

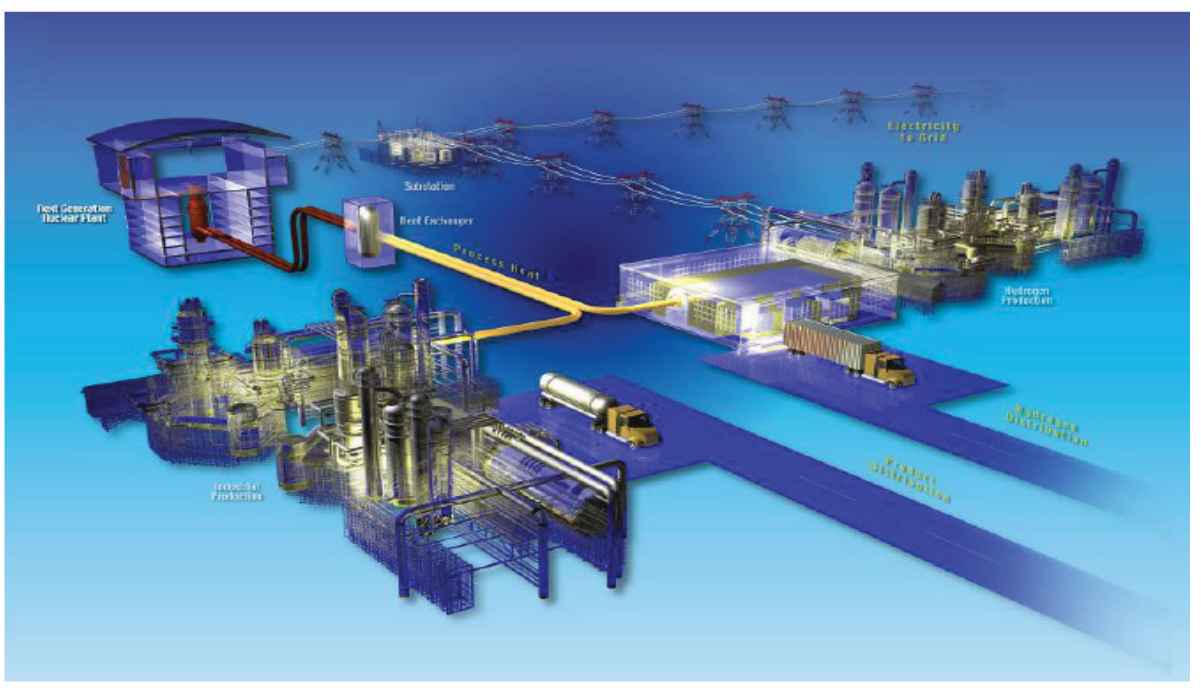

The INL is a U.S. Department of Energy National Laboratory operated by Battelle Energy Alliance 
INL/EXT-08-14729

Rev. 1

\title{
COMBINE7.1 - A Portable ENDF/B-VII.0 Based Neutron Spectrum and Cross-Section Generation Program
}

\author{
W. Y. Yoon \\ R. A. Grimesey \\ D. W. Nigg \\ R. L. Curtis
}

August 2009

\begin{abstract}
Idaho National Laboratory Idaho Falls, Idaho 83415
\end{abstract}

http://www.inl.gov

Prepared for the

U.S. Department of Energy

Office of Nuclear Energy

Under DOE Idaho Operations Office

Contract DE-AC07-05ID14517 


\section{DISCLAIMER}

This information was prepared as an account of work sponsored by an agency of the U.S. Government. Neither the U.S. Government nor any agency thereof, nor any of their employees, makes any warranty, expressed or implied, or assumes any legal liability or responsibility for the accuracy, completeness, or usefulness, of any information, apparatus, product, or process disclosed, or represents that its use would not infringe privately owned rights. References herein to any specific commercial product, process, or service by trade name, trade mark, manufacturer, or otherwise, does not necessarily constitute or imply its endorsement, recommendation, or favoring by the U.S. Government or any agency thereof. The views and opinions of authors expressed herein do not necessarily state or reflect those of the U.S. Government or any agency thereof. 

Nuclear Energy

COMBINE7.1-A Portable ENDF/B-VII.0 Based Neutron Spectrum and Cross-Section Generation Program

INL/EXT-08-14729

$\operatorname{Rev} 1$

August 2009

Approved by:

woo your

Woo Yoon

Author

$\frac{\text { NCaa }}{\text { Diane V. Croon }}$

VHTR TDO Project Manager
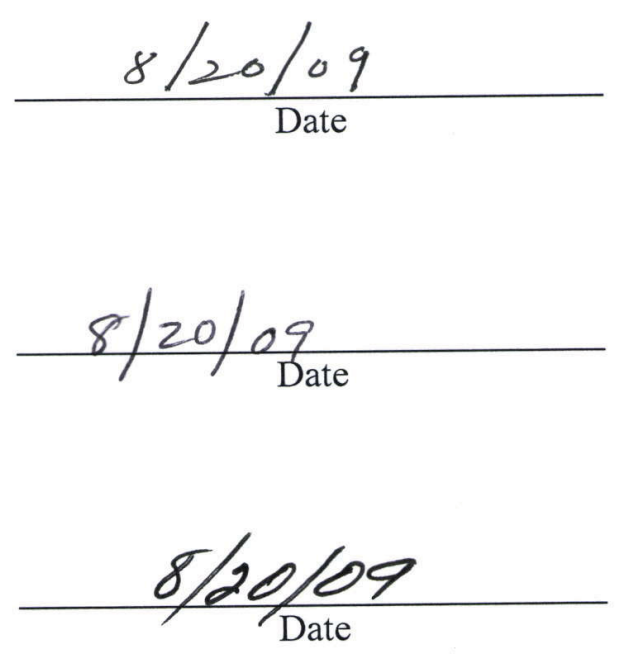

VHTR TDO Deputy Technical Director 



\begin{abstract}
COMBINE7.1 is a FORTRAN 90 computer code that generates multigroup neutron constants for use in the deterministic diffusion and transport theory neutronics analysis. The cross-section database used by COMBINE7.1 is derived from the Evaluated Nuclear Data Files (ENDF/B-VII.0). The neutron energy range covered is from $20 \mathrm{MeV}$ to $1.0 \mathrm{E}-5 \mathrm{eV}$. The Los Alamos National Laboratory NJOY code is used as the processing code to generate a 167 finegroup cross-section library in MATXS format for Bondarenko self-shielding treatment. Resolved resonance parameters are extracted from ENDF/B-VII.0 File 2 for a separate library to be used in an alternate Nordheim self-shielding treatment in the resolved resonance energy range. The equations solved for energy dependent neutron spectrum in the 167 fine-group structure are the B-3 or B-1 approximations to the transport equation. The fine group cross sections needed for the spectrum calculation are first prepared by Bondarenko selfshielding interpolation in terms of background cross section and temperature. The geometric lump effect, when present, is accounted for by augmenting the background cross section. Nordheim self-shielded fine group cross sections for a material having resolved resonance parameters overwrite correspondingly the existing self-shielded fine group cross sections when this option is used. The fine group cross sections in the thermal energy range are replaced by those selfshielded with the Amouyal/Benoist/Horowitz method in the three region geometry when this option is requested. COMBINE7.1 coalesces fine group cross sections into broad group macroscopic and microscopic constants. The coalescing is performed by utilizing fine-group fluxes and/or currents obtained by spectrum calculation as the weighting functions. The multigroup constant may be output in any of several standard formats including ANISN $14^{* *}$ free format, CCCC ISOTXS format, and AMPX working library format. ANISN-PC, a onedimensional, discrete-ordinate transport code, is incoprated into COMBINE7.1. As an option, the 167 fine-group constants generated by COMBINE portion in the program can be used to cacluate regionwise spectra in the ANISN portion, all internally to reflect the one-dimensional transport correction. Results for the criticality validation calculations are included as a part of verification and validation.
\end{abstract}




\section{ACKNOWLEDGMENTS}

A number of individuals contributed to the successful development of the COMBINE7.1 code, which was derived from COMBINE5 and 6 codes . Gilbert Singer provided an initial standard FORTRAN version of the CVI free-format input processing routines used in COMBINE/PC. Jerry Judd, J. Russell Johnson, Blair Briggs, and Bruce Schnitzler contributed to the programming of several routines. Dr. D. Parsons, formerly with Idaho National Laboratory Laboratory (INL), is greatly appreciated for the use of ANISN/PC. Several members of the INL/University/Industry PC software review committee provided useful comments and tested the software prior to its release. Dr. Hans Gougar extensively tested the COMBINE7.1 beta versions and his inputs were invaluable. The Next Generation Nuclear Program at INL deserves a special mention for helping realize the current code version. 


\section{CONTENTS}

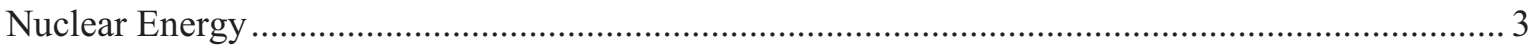

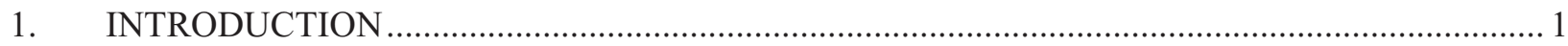

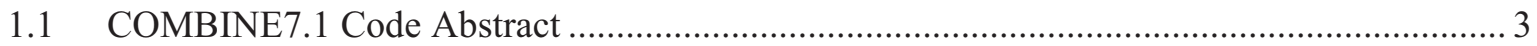

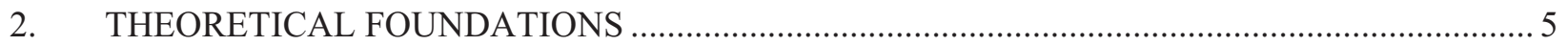

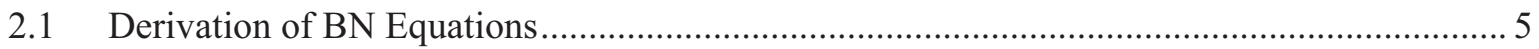

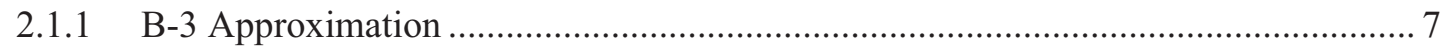

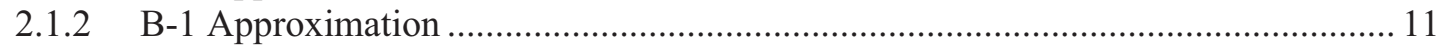

2.1.3 Convergence on Gauss-Seidel Iterative Scheme....................................................... 12

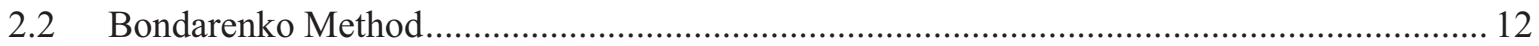

2.2.1 Fine-Group Cross Section Generation in Bondarenko Format ................................ 15

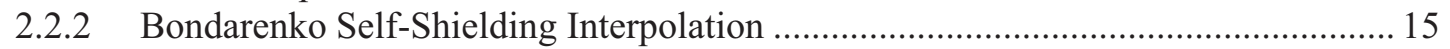

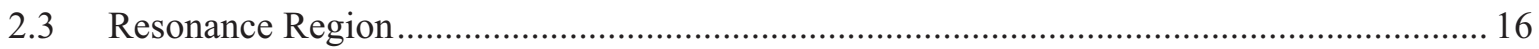

2.3.1 Resolved Resonances_-Nordheim Numerical Method............................................ 16

2.3.2 Doppler Broadening of Resolved Resonance Parameters ........................................ 19

2.3.3 Determination of $E_{1} / E_{2}$ and the Number of Meshes for a Resonance........................ 20

2.3.4 Absorber Narrow Resonance- Moderator Asymptotic Flux Approximation............ 21

2.3.5 Self-Shielding Elastic Scattering Transfer Matrix .................................................... 21

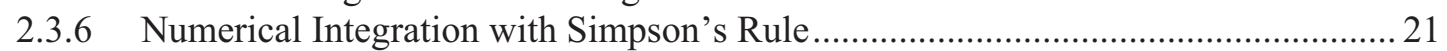

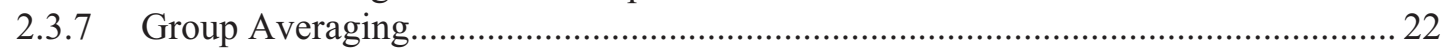

2.3.8 Dancoff-Ginsburg Correction Factor Calculation.............................................. 22

2.4 Computation of Thermal Disadvantage Factors................................................................... 25

2.4.1 Slab Geometry Disadvantage Weights............................................................... 27

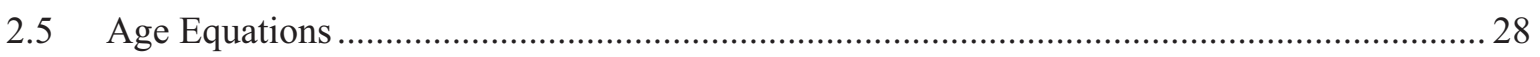

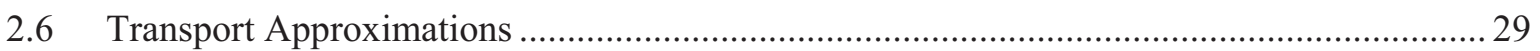

3. ONE-DIMENSIONAL TRANSPORT CALCULATION …......................................................... 31

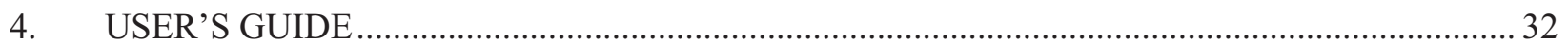

4.1 Derivation of Diffusion Coefficient and Transport Cross Section ........................................ 32

4.2 Summary Edits for Broad Group Constants............................................................................ 33

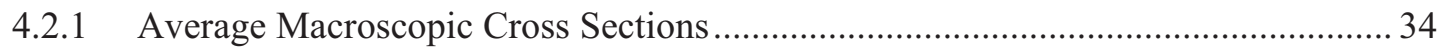

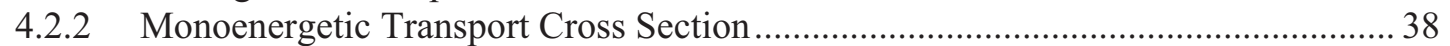

4.2.3 Average Microscopic Cross Sections......................................................................... 38

4.2.4 Effective Diffusion Theory Constants (Blackness Theory) ....................................... 39

4.2.5 Mixed Number Density (MIND) Constants.......................................................... 40

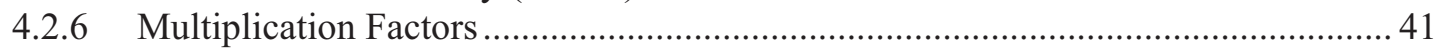

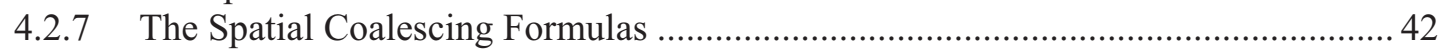

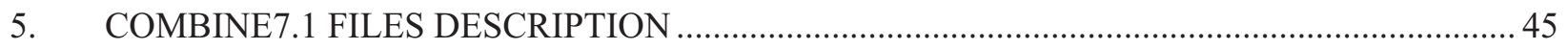

6. COMBINE7.1 INPUT DATA FILE ORGANIZATION ….................................................... 46

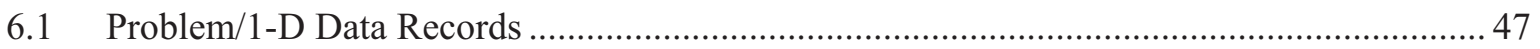

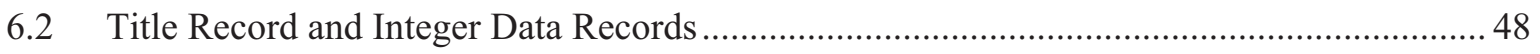




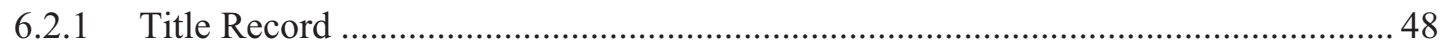

6.2.2 Problem Basic Control Information Record 1010101 ............................................... 48

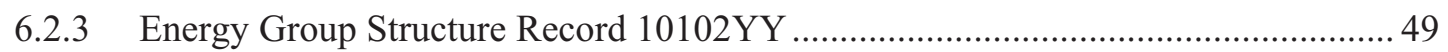

6.2.4 Resonance Calculation Option Record 102010Y. ................................................. 49

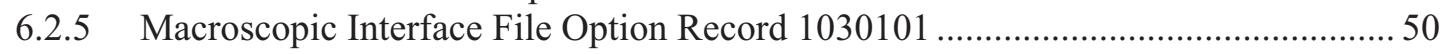

6.2.6 Microscopic Interface File Option Record 10302XX ............................................... 51

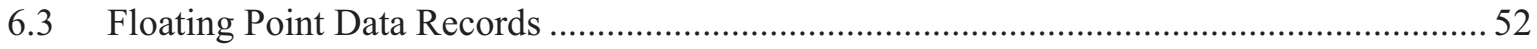

6.3.1 General Floating Point Data Record 1041001 ......................................................... 52

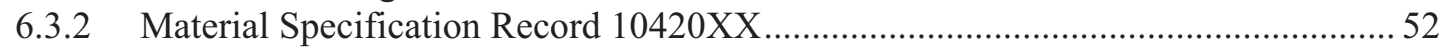

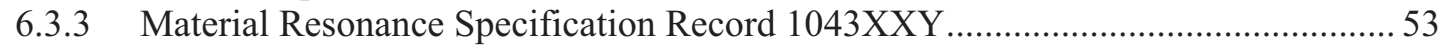

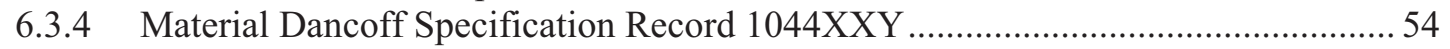

6.3.5 Material General Resonance Data Record 10450XX …........................................ 54

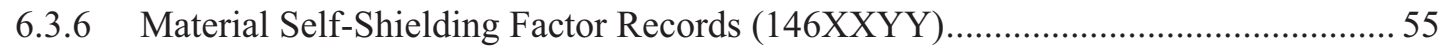

6.3.7 Broad Group Buckling Factor Records (14700YY) ............................................... 55

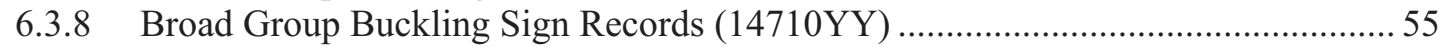

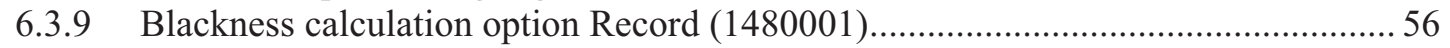

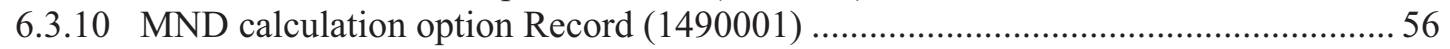

6.4 Mixed Integer and Floating Point Data Records ............................................................... 57

6.4.1 ABH REGION DESCRIPTION RECORDS 2010401 .......................................... 57

6.4.2 ABH Material Description for Each Region Record 2015XYY ...............................5 57

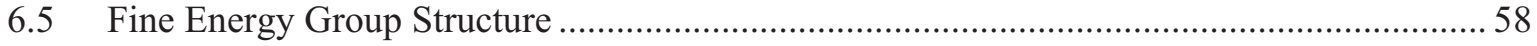

6.6 Materials in the "MATXS" Fine Group Cross Section Library and the "RERES"

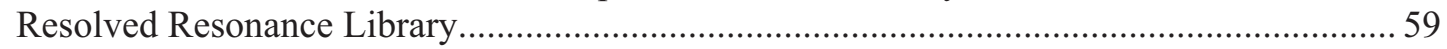

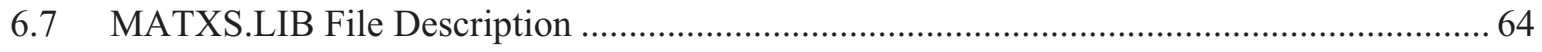

6.8 Description of Resolved Resonance Library (reres.lib) Format.......................................... 68

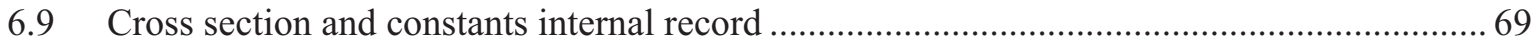

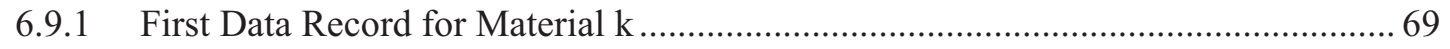

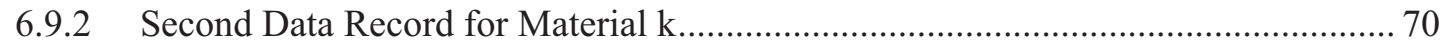

6.10 Thermal $S(\alpha, \beta)$ and Free Scattering Cross Section Internal Record ................................... 71

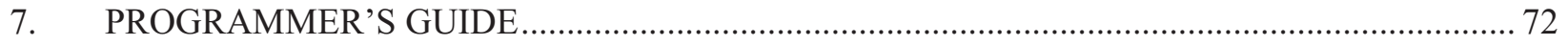

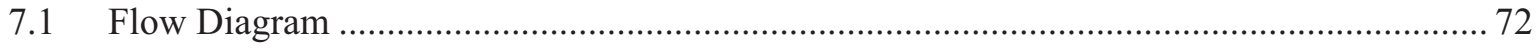

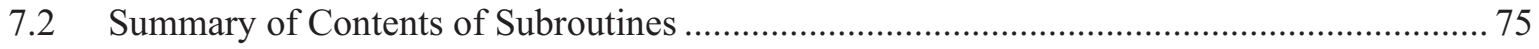

7.2.1 Program COMBINE7.1 and Supporting Subroutines ............................................ 75

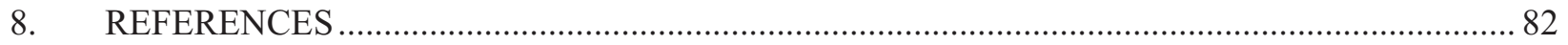

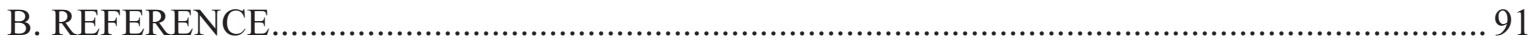

ISOTXS - Nuclide (Isotope) Ordered, Multigroup Neutron Cross-Sections .................................. 95

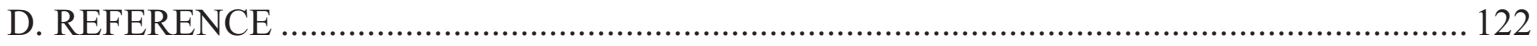

\section{FIGURES}

Figure 1. COMBINE7.1 implementation scheme. 3 
Figure 2. An example of Doppler broadened resonance cross sections.

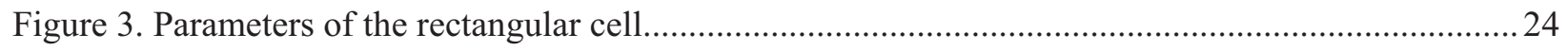

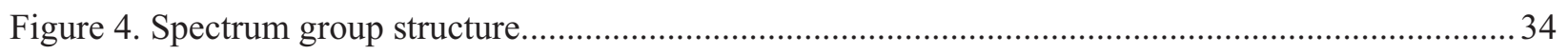

\section{TABLES}

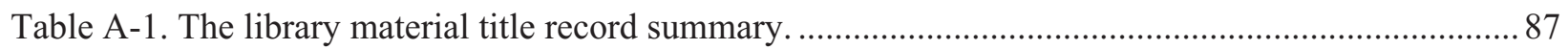

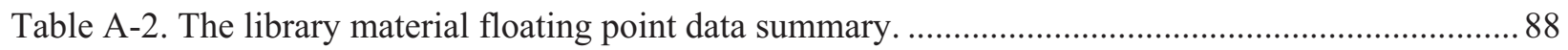

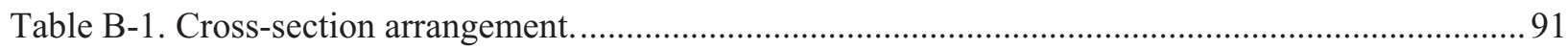

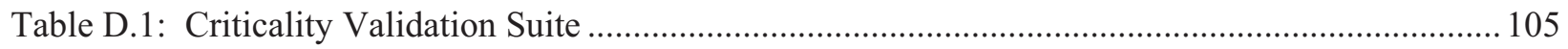

Table D.2: Calculated keff eigenvalues against the measured keff $=1.000$ B1 and No Sn Transport Correction .....

Table D.3: Calculated keff eigenvalues against the measured keff $=1.000 \mathrm{~B} 1$ and Sn Transport Correction.

Table D.4: Calculated keff eigenvalues against the measured keff $=1.000 \mathrm{~B} 3$ and No Sn Transport Correction.

Table D.5: Calculated keff eigenvalues against the measured keff $=1.000$ B3 and Sn Transport Correction 


\section{ACRONYMS}

$\begin{array}{ll}\text { ABH } & \text { Amouyal/Benoist/Horowitz } \\ \text { ENDF } & \text { Evaluated Nuclear Data Files } \\ \text { HTR } & \text { High Temperature Reactor } \\ \text { INL } & \text { Idaho National Laboratory } \\ \text { MLBW } & \text { Multilevel Breit-Wigner } \\ \text { NGNP } & \text { Next Generation Nuclear Plant } \\ \text { PBMR } & \text { Pebble Bed Modular Reactor } \\ \text { R-M } & \text { Reich-Moore } \\ \text { SLBW } & \text { single level Breit-Wigner } \\ \text { MND } & \text { mixed number density }\end{array}$




\section{COMBINE7.1-A Portable ENDF/B-VII.0 Based Neutron Spectrum and Cross-Section Generation Program}

\section{INTRODUCTION}

COMBINE7.1 is a FORTRAN 90 computer code for generation of spectrum-averaged, multigroup, neutron, cross-section data suitable for use in the deterministic diffusion and transport theory reactor design analysis. The cross-section database used by COMBINE7.1 is derived from Evaluated Nuclear Data Files (ENDF/B-VII. $0^{1}$ ). The neutron energy ranging from $20 \mathrm{MeV}$ to $1.0 \mathrm{E}-5 \mathrm{eV}$ is treated by a total of 167 discrete energy groups. The equations solved for the energy-dependent neutron spectrum are the $B_{3}$ or $B_{1}$ approximations to the transport equation.

COMBINE7.1 has evolved from its predecessor Versions 5 and 6 . The earlier version, COMBINE/PC - a Portable ENDF/B Version 5 Neutron Spectrum and Cross-Section Generation Program $^{2}$ (COMBINE5) - combined the preexisting neutron spectrum codes, PHROG $^{3}$ (fast) and INCITE $^{4}$ (thermal spectrum), previously developed at Idaho National Laboratory (INL), by suitably integrating them into a single package, thus, the name COMBINE. The cross-section database used by COMBINE5 is derived from the ENDF/B- $\mathrm{V}^{5}$. In the fast spectrum calculation, the $B_{3}$ spherical harmonics equations are solved by Gauss elimination and the $B_{1}$ spherical harmonics equations are solved simultaneously, beginning with the highest energy group. In INCITE, the $B_{1}$ thermal equations have been cast in the form of a normalized Gauss-Seidel iterative scheme where extrapolation and iteration in both directions have been included as options. The fast and thermal energy ranges are coupled by using slowing down theory and energy-dependent scattering and spectra data to generate average transfer cross sections from the PHROG broad groups into INCITE broad groups. The results are then normalized to the total scatter below the fast range cutoff as produced by PHROG.

The 1990 release of ENDF/B-VI nuclear data ${ }^{6}$ of ENDF-6 format created problems for the INL's Version 5 processing codes used to generate cross-section libraries for COMBINE5, requiring major revisions of these codes. An alternative to the modifications of the INL's processing codes was to utilize the NJOY code system ${ }^{7}$ developed at the Los Alamos National Laboratory. NJOY optionally processes all resonance self-shielding through the Bondarenko approximation. In the Bondarenko model, ${ }^{8}$ the narrow resonance (NR) approximation and the $B_{\mathrm{N}}$ approximation for large systems are invoked. ${ }^{9}$ Two cross section libraries were generated by NJOY for the fast ( 72 group) and thermal (101 energy points) spectrum calculations, maintaining the same energy structures as in COMBINE5. The fast cross section library was based on sets of background cross sections and temperatures for Bondarenko self-shielding interpolation. The geometric effects of fuel lump and lattice are accounted for by the combination of Rational Approximation and Dancoff-Ginsburg correction, including a double heterogeneity due to fuel grains present within the macroscopic fuel lump by augmenting the background cross section. The weakness of the Bondarenko model occurs for thermal reactor analysis in the "epithermal" energy region from about $4 \mathrm{eV}$ to around 200 to $300 \mathrm{eV} .{ }^{10}$ In this region, the resonances are no longer "narrow" and the flux shape given by Bondarenko method is no longer sufficiently accurate. An alternate approach is the Nordheim resonance integral treatment method. ${ }^{11}$ A separate resolved resonance library was also created, including formats in single level Breit-Wigner (SLBW), Multilevel Breit-Wigner (MLBW), Reich-Moore (R-M), and no resonance, extracted directly from ENDF/B-VI nuclear data files to be used in the Nordheim self-shielding treatment in the resolved resonance energy range. The Version 5 code was modified to handle the new cross section and resolved resonance libraries. The new code was designated as COMBINE6. ${ }^{12}$ The ENDF/B-VI derived thermal cross-section library is based on the infinite dilution and temperature dependence is only present if thermal scattering data is available for an isotope. This format of the thermal cross-section library is same as that of COMBINE5's. The INCITE portion of COMBINE5 is therefore fully retained, including the Amouyal/Benoist/Horowitz (ABH) option for selfshielding in the thermal energy range. The energy range treated is from $0.001 \mathrm{eV}$ to $16.905 \mathrm{MeV}$ spanned 
by a total of 166 discrete energy points/groups. A routine was also retained to calculate a DancoffGinsburg correction factor in conjunction with the resonance calculation for either a rectangular or hexagonal arrangement of cylindrical absorber pins. Several routines to process MLBW and R-M resonance formats were adopted from the NJOY code. Nordheim self-shielded cross sections, when requested for a material having resolved resonance parameters, overwrite the corresponding multigroup Bondarenko self-shielded cross sections. An iterative scheme was also introduced for better fission-rate weighted $\chi$ values. In both COMBINE5 and COMBINE6, the energy boundary between the fast spectrum module and thermal spectrum module can be chosen by the user at one of the eight overlapping mutual energy group boundaries, the highest being $2.3824 \mathrm{eV}$ and the lowest $0.414 \mathrm{eV}$. No resonance treatment is considered below the chosen energy boundary. No thermal upscattering is allowed into the energy above the highest boundary. Thermal upscattering does not take place between the highest $(2.3824 \mathrm{eV})$ and chosen boundary of less energy, even though it is possible from below to above the boundary when the boundary is below the highest. The adequate self-shielding treatment of resonances in the thermal energy range is critical where thermal upscattering is also significant because of high operating temperature. Overlap and interference effect of resonances among admixed absorbing isotopes was not accounted.

COMBINE7.1 has been modified to resolve the above issues. The $B_{1}$ and $B_{3}$ transport algorithms for the full range multigroup treatment, including thermal upscattering, were identified and implemented to unify fast and thermal spectrum calculations. The neutron energy ranging from $20 \mathrm{MeV}$ to $1.0 \mathrm{E}-5 \mathrm{eV}$ is treated by a total of 167 discrete energy groups, adding one more group (20-16.905 MeV) to the previous versions' group structure. The thermal upscattering takes place between $1.0 \mathrm{E}-5 \mathrm{eV}$ and $5.0435 \mathrm{eV}$ (high enough for most reactor applications) with no energy boundary restriction. Up to g-wave resonances are now treated to obtain the elastic scattering cross section, although the isotropic scattering is still used for energy transfer. In the Nordheim resonance treatment, up to three admixed resonance absorbers can be present to account for the resonance overlap and interference effect. Thermal upscattering was also added in the resonance treatment solution. The interpolation algorithm in the Bondarenko scheme to self-shield cross sections has been modified for improvement and revamped to afford additional temperature-onlydependent thermal scatterings, free and/or $\mathrm{S}(\alpha, \beta)$. The interpolation is now performed first for the background cross sections at each temperature and the generated cross sections for the set of preselected temperatures are then interpolated for the problem-dependent temperature. Negative buckling algorithms have been implemented in addition to a real $B$ : one with the positive imaginary $B$ and the other with the negative imaginary $B$. Previously, only the positive and real $B$ of cosine spatial shape was allowed. The mixed use of three different modes of buckling in the broad group wise input is implemented. All resonances are Doppler broadened by the method called "kernel broadening" as practiced in NJOY, thus eliminating the need to evaluate Doppler line functions ( $\psi$ and $\chi$ ) previously calculated in the SLBW treatment. The GAM-1 resolved-resonance calculation routine, ${ }^{13}$ a fast alternative to the much more accurate but time consuming Nordheim numerical method, was scrapped since speed is no longer a factor. Many of the INCITE routines were removed or combined with PHROG due to the unified energy scheme.

The necessity of better treat spatial self-shielding and reflector effects when dealing with neutronoptically thin reactors like the annular core design of gas-cooled, graphite-moderated high temperatures (HTRs) such as proposed for the Next Generation Nuclear plat (NGNP) requires the transport correction in generating the cross sections for use in reactor physics. For this, 1-D discrete-ordinate transport code, ANISN-PC, ${ }^{14}$ is incorporated into COMBINE7.1 to reflect one-dimensional (1-D) transport correction. With this incorporation, a fuel such as TRISO/PEBBLE can be also better modeled to generate cross sections for the pebble bed reactor (PBR) analyses. The 167 fine-group constants generated by COMBINE for multiregions via stack cases are fed into ANISN to calculate regionwise spectra, which are used in the COMBINE to generate the regionwise spatial self-shielded broad group constants. The detail of ANISN code will be deferred to its manual. Figure 1 shows the COMBINE7.1 implementation scheme. 


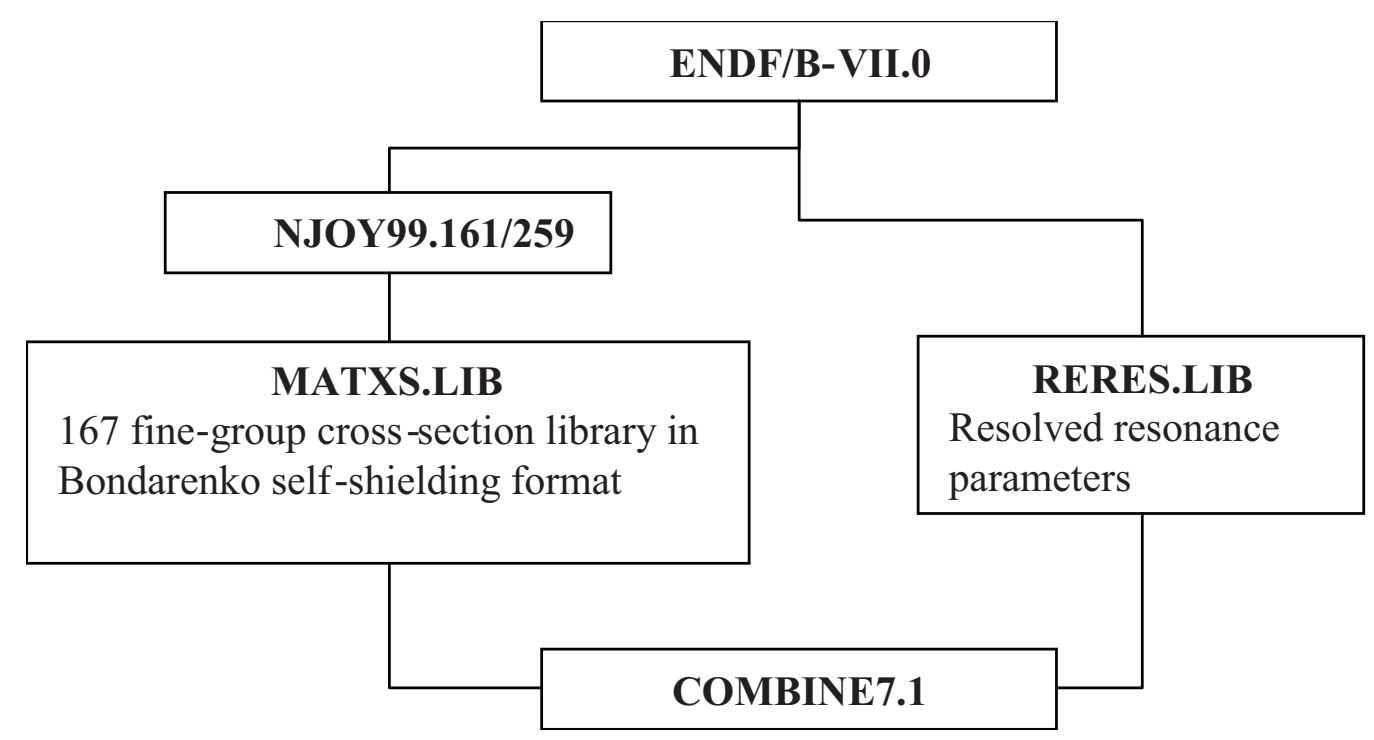

Figure 1. COMBINE7.1 implementation scheme.

ENDF/B-VII.0 nuclear data was released in December 2006 using the same ENDF-6 format. ${ }^{1}$ The cross-section database is now derived entirely from ENDF/B-VII.0 nuclear data with the exception of $\mathrm{Zr}$ element cross-section data, which is based on ENF/B-VI.8 nuclear data. The NJOY99.259 version ${ }^{15}$ was used to generate a 167 fine-group cross-section library, MATXS.LIB, in MATXS format (Bondarenko format) on sets of background cross sections and temperatures including thermal scattering cross sections. This cross-section library includes damage, heating, kerma, and gas production cross sections in addition to those vector and matrix cross sections required for transport calculations. Only those materials designated by ENDF/B File 7 formats as moderators and included in NJOY99.259 contain thermal $\mathrm{S}(\alpha, \beta)$ scattering matrices. The nonmoderating materials in the library possess free thermal scattering matrices. A separate resolved resonance library, RERES.LIB, was also created that includes formats in SLBW, MLBW, R-M, and no resonance, extracting all resolved resonance parameters directly from ENDF/BVII.0 nuclear data files. Along with the cross section library in Bondarenko format and the resolved resonance data library, the upgraded COMBINE version is now called COMBINE7.1.

As the code has evolved, further improvements are expected in the future. It is also anticipated that the next revision would include calculation experiences involving PBR.

\subsection{COMBINE7.1 Code Abstract}

\section{Program Identification: COMBINE7.1}

2. Description of Problem: COMBINE7.1 is a FORTRAN 90 computer code that generates multigroup neutron constants for use in the deterministic diffusion and transport theory neutronics analysis. The cross-section database used by COMBINE7.1 is derived from ENDF/B-VII.0. The neutron energy range covered is from $20 \mathrm{MeV}$ to $1.0 \mathrm{E}-5 \mathrm{eV}$. The equations solved for energy dependent neutron spectrum are the B-3 or B-1 approximations to the transport equation. It is also capable of performing a $1 \mathrm{D}$ discrete-coordinates transport calculation for multiregions to generate regionwise broad group constants.

3. Method of Solution: The energy range is broken into 167 fine groups for the spectrum calculation. The resulting matrix of flux equations is then solved using standard numerical techniques to obtain the spectral weight function for generation of group-averaged constants. An alternate resolved resonance shielding of the fine-mesh cross section data is performed using the Nordheim method. An option to treat thermal self shielding using the $\mathrm{ABH}$ method is provided. User-defined self-shielding 
factors (from an auxiliary transport code such as ANISN/PC) may be entered. Calculated cross sections may be issued in any of several standard formats.

4. Related Material: A MATXS library file and a resolved resonance parameter library file are provided with the code package in ASCII format. Processors to convert the MATXS file to the required binary format or to convert the binary file to the ASCII file are provided.

cv700.190: COMBINE-7 Cycle 0 Fortran 90 source program.

It requires matxs.lib and reres.lib to run a problem.

matxs.asc: 167 fine-group cross section library in ascii format

matxs.lib: 167 fine-group cross section library in binary format

reres.lib: resolved resonance library in ascii format

matxscv.f: Fortran program to convert matxs.asc (ascii file) to matxs.lib (binary file)

matxsca.f: Fortran program to convert matxs.lib to matxs.asc

anisn.pdf: ANISN/PC Manual (pdf file).

5. Restrictions: COMBINE7.1 is written to function properly on 32-bit word length machine. Accuracy to four or five decimal places in the computed spectrum and cross sections may be expected.

6. Computers: COMBINE7.1 is written in standard FORTRAN with the objective of total machine independence. The program has been successfully compiled by the Intel Visual Fortran Compiler (Version 10) and by the Lahey/Fujitsu Fortran compiler (LF95) on the Windows 32-bit platform.

7. Run Time: Variable from a few seconds to several minutes on PC-class hardware, depending primarily on the number and type of resonance self-shielding calculations required for a particular case.

8. Programming Language: Standard FORTRAN 90.

9. Operating System: COMBINE7.1 has successfully functioned under Windows and Linux operating systems.

10. Machine Requirements: As stated in item 6, Computers.

11. Authors: W. Y. Yoon

R. A. Grimesey

D. W. Nigg

R. L. Curtis

Idaho National Laboratory

Battelle Energy Alliance

P.O. Box 1625

Idaho Falls, ID 83415 


\section{THEORETICAL FOUNDATIONS \\ 2.1 Derivation of BN Equations}

The equations solved by COMBINE are the energy-dependent $B_{1}$ or $B_{3}$ spherical harmonic approximations to the transport equation originally developed by D. S. Selengut. ${ }^{16}$ A derivation of the $B_{\mathrm{N}}$ spherical harmonics equations is straightforward, but rather tedious because of the complicated expansion integrals. The physical model is a homogeneous, critical, 1-D bare slab core. As such, the timeindependent, energy dependent, Boltzmann neutron transport equation in plane geometry may be written

$\mu \frac{\partial \Phi(x, \mu, E)}{\partial x}+\Sigma_{t}(x, E) \Phi(x, \mu, E)=\iint \Sigma_{s}\left(x ; \Omega^{\prime}, E^{\prime} \rightarrow \Omega, E\right) \Phi\left(x, \mu^{\prime}, E^{\prime}\right) d \Omega^{\prime} d E^{\prime}+Q(x, \mu, E)$

where: $\Phi(x, E, \mu)$ is the neutron flux in the direction $\mu=\cos \theta$ relative to the 1-D $\mathrm{x}$ axis of the slab at energy $E$ and position $x$ and $\boldsymbol{\Omega}$ and $\boldsymbol{\Omega}$ ' are the azimuthal angles associated with $\mu$ and $\mu^{\prime}$, respectively; $\Sigma_{t}$ is the total cross section; and $\Sigma_{s}\left(x ; \Omega^{\prime}, E^{\prime} \rightarrow \Omega, E\right)$ is the neutron transfer cross section, including the multiplicity of neutrons emitted in each reaction event. Q represents the independent (or extraneous) neutron sources that are not dependent on the neutron density of the system. All cross sections are macroscopic here. It will be assumed that the angular variation of the transfer cross section, $\Sigma_{\mathrm{s}}$, depends only on the scattering angle, $\mu_{0}=\mathbf{\Omega} \cdot \mathbf{\Omega}$ '. Then $\Sigma_{\mathrm{s}}$ may be expanded in a set of Legendre polynomials of $\mu_{0}$; thus,

$\Sigma_{S}\left(x ; \Omega^{\prime}, E^{\prime} \rightarrow \Omega, E\right)=\sum_{l=0}^{\infty} \frac{2 l+1}{4 \pi} \Sigma_{s l}\left(x ; E^{\prime} \rightarrow E\right) P_{l}\left(\mu_{0}\right)$,

where the expansion coefficients $\Sigma_{s l}\left(x ; E^{\prime} \rightarrow E\right)$ are given by

$$
\Sigma_{s l}\left(x ; E^{\prime} \rightarrow E\right)=2 \pi \int_{-1}^{1} \Sigma_{s}\left(x ; E^{\prime} \rightarrow E, \mu\right) P_{l}(\mu) d \mu
$$

If the expansion of Equation (3) is inserted into Equation (1), use of the addition theorem for Legendre polynomials and integration over azimuthal angles gives

$$
\mu \frac{\partial \Phi(x, \mu, E)}{\partial x}+\Sigma_{t}(x, E) \Phi(x, \mu, E)=\sum_{l=0}^{\infty} \frac{2 l+1}{2} P_{l}(\mu) \int_{E^{\prime}} \Sigma_{s}\left(x, E^{\prime} \rightarrow E\right) \int_{-1}^{1} \Phi\left(x ; \mu^{\prime}, E^{\prime}\right) P_{l}\left(\mu^{\prime}\right) d \mu^{\prime} d E^{\prime}+Q(x, \mu, E) .
$$

The basis of the $B_{\mathrm{N}}$ method, as a means of estimating within-group neutron fluxes, is to assume the spatial distribution to be independent of neutron energy in plane geometry,

$\Phi(x, \mu, E)=e^{-i B x} \psi(B, \mu, E)$,

where only the real part of $e^{-i B x}$ satisfies the spatial boundary conditions for the scalar flux. The complex form of the spatial dependence is necessary for the equations to remain homogeneous. In Equation (5) the spatial dependence of the flux is eliminated by assuming that the solution is separable in $(E, \mu)$ and $\mathrm{x}$ and characterizing the spatial dependence of each of the variables by a simple buckling mode. If Equation (5) is inserted into the neutron transport Equation (4) with $Q(x, \mu, E)$ replaced by an isotropic fission source, $\frac{1}{2} \chi(E) e^{-i B x}$, and the transfer cross section is now designated as $\Sigma_{S l}$ signifying the absence of fission contribution, the result is 


$$
\begin{aligned}
& \Sigma_{t}(E)\left(1-\frac{i B \mu}{\Sigma_{t}}\right) \psi(B, \mu, E) \\
& =\sum_{l=0}^{\infty} \frac{2 l+1}{2} p_{l}(\mu) \int \Sigma_{S l}\left(E^{\prime} \rightarrow E\right) \int_{-1}^{1} \psi\left(B, \mu^{\prime}, E^{\prime}\right) P_{l}\left(\mu^{\prime}\right) d \mu^{\prime} d E^{\prime}+\frac{1}{2} \chi(E) .
\end{aligned}
$$

The neutron source is assumed to have the same spatial dependence as the flux. This assumption is also necessary in order for the equations to remain homogeneous. The source term, $1 / 2 \chi(E)$, in

Equation (6) is the result of Legendre polynomial expansion and truncation to the first term, assuming the fission source to be isotropic in lab coordinates. It is also noted that independent (or extraneous) neutron sources, which are not dependent on the neutron density of the system, are not included in this equation. Equation (6) could be multiplied by $P_{n}(\mu)$ and integrated over $\mu$ from -1 to 1 to obtain equations satisfied by the Legendre components of $\psi(B, \mu, E)$. More rapid convergence of the expansion is achieved, however, when equation (6) is divided by $\Sigma_{t}\left(1-i B \mu / \Sigma_{t}\right)$, multiplied by $P_{n}(\mu)$, and then integrated to obtain, for $n=0,1,2 \ldots$,

$$
\begin{aligned}
& \phi_{n}(B, E) \\
& =\sum_{l=0}^{\infty}(2 l+1) \frac{A_{n l}(B, E)}{\Sigma_{t}(E)} \int \Sigma_{s l}\left(E^{\prime} \rightarrow E\right) \phi_{l}\left(B, E^{\prime}\right) d E^{\prime}+\frac{A_{n 0}(B, E)}{\Sigma_{t}(E)} \chi(E),
\end{aligned}
$$

using the following definitions:

$(i)^{n} \phi_{n}(B, E) \equiv \int_{-1}^{1} \psi(B, \mu, E) P_{n}(\mu) d \mu$. when $B$ is real,

$\phi_{n}(B, E) \equiv \int_{-1}^{1} \psi(B, \mu, E) P_{n}(\mu) d \mu$. when $B$ is imaginary $(\mathrm{B}= \pm \mathrm{iC}$, where $\mathrm{C}$ is real),

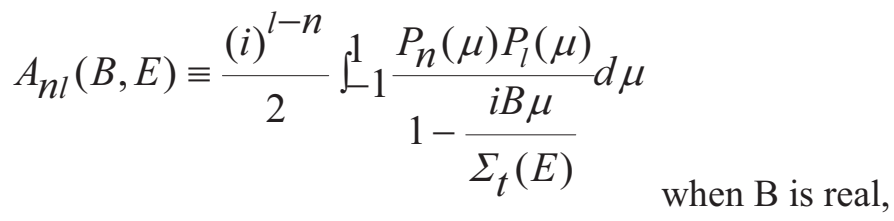

$$
A_{n l}(B, E) \equiv \frac{1}{2} \int_{-1}^{1} \frac{P_{n}(\mu) P_{l}(\mu)}{1-\frac{i B \mu}{\Sigma_{t}(E)}} d \mu
$$

when B is imaginary.

When $\mathrm{n}=0$, for example, the value $P_{0}(\mu)$ is 1 ; hence, it follows from Equation (8) that $\Phi_{0}(B, E)$ is simply the total flux at E.

From Equations (10) and (11), the following symmetry and recursion relations can be derived $(i)^{n-l} A_{n l}=(i)^{l-n} A_{l n}$ when B is real,

$A_{n l}=A_{l n}$ when B is imaginary,

$\frac{1}{B / \Sigma_{t}}(2 l+1) A_{n l}-(l+1) A_{n, l+1}+l \cdot A_{n, l-1}=\frac{\delta_{n l}}{B / \Sigma_{t}}$ when B is real, 
$\frac{1}{C / \Sigma_{t}}(2 l+1) A_{n l}+(l+1) A_{n, l+1}+l \cdot A_{n, l-1}=\frac{\delta_{n l}}{C / \Sigma_{t}}$ when $\mathrm{B}=\mathrm{iC}$ and $\mathrm{C}$ is real,

$\frac{1}{C / \Sigma_{t}}(2 l+1) A_{n l}-(l+1) A_{n, l+1}-l \cdot A_{n, l-1}=\frac{\delta_{n l}}{C / \Sigma_{t}}$ when $\mathrm{B}=-\mathrm{iC}$ and $\mathrm{C}$ is real.

where $\delta_{n l}$ is the Kronecker delta.

Now, all of the $\Phi_{n}$ in Equation (7) is real quantity. $l$ is the limit for the Legendre expansion of the differential scattering cross section; the expansion is truncated at $l+1$. However, $l$ does not determine the order of the $B_{\mathrm{N}}$ equations. The integral in Equation (7) may be solved to any order $n$ independent of the value of $l$. As $n$ is increased beyond $l$, no particular advantage is gained in a spectrum code like COMBINE because the ultimate purpose of the solution fluxes is to collapse the cross sections to a few group libraries for spatial transport codes. For $n>l$ there are no cross sections in the library because the elastic scattering matrix was truncated at $l+1$. Therefore, there is no reason to include higher order fluxes than $n=3$ in the $B_{3}$ solution. The same argument pertains to the $B_{1}$ approximation; in this case $n$ is truncated at 1 .

The coefficients, $A_{n l}$, will become a function of $\alpha=B / \Sigma_{t}$ and $A_{00}=\beta=\frac{\tan ^{-1} \alpha}{\alpha}$ when B is real or $\alpha=C / \Sigma_{t}$ and $A_{00}=\beta=\frac{\tanh ^{-1} \alpha}{\alpha}$ when $\mathrm{B}= \pm \mathrm{iC}$ with a real $\mathrm{C}$.

\subsubsection{B-3 Approximation}

Evaluation of Equation (10) for the $B_{3}$ approximation, limiting $l$ and $n$ to 3 , is straightforward but rather tedious for the higher order coefficients.

$$
\begin{aligned}
& A_{00}=\beta=\frac{\tan ^{-1} \alpha}{\alpha} \\
& A_{01}=-\frac{1-\beta}{\alpha} \\
& A_{02}=-\frac{3}{2} \frac{(1-\beta)}{\alpha^{2}}+\frac{\beta}{2} \\
& A_{11}=-\frac{1-\beta}{\alpha^{2}} \\
& A_{12}=-\frac{3}{2} \frac{(1-\beta)}{\alpha^{3}}-\frac{\beta}{2 \alpha} \\
& A_{22}=\frac{1}{4 \alpha}\left[\left(\frac{9}{\alpha^{3}}+\frac{6}{\alpha}+\alpha\right) \beta-\frac{3}{\alpha}-\frac{9}{\alpha^{3}}\right] \\
& A_{03}=\frac{1}{2 \alpha}\left[\left(\frac{5}{\alpha^{2}}+3\right) \beta-\frac{4}{3}-\frac{5}{\alpha^{2}}\right] \\
& A_{13}=\frac{1}{2 \alpha^{2}}\left[\left(\frac{5}{\alpha^{2}}+3\right) \beta-\frac{4}{3}-\frac{5}{\alpha^{2}}\right]
\end{aligned}
$$




$$
\begin{aligned}
& A_{23}=-\frac{1}{4 \alpha}\left[\frac{15}{\alpha^{4}}+\frac{9}{\alpha^{2}}+\frac{4}{3}-\left(\frac{15}{\alpha^{4}}+\frac{14}{\alpha^{2}}+3\right) \beta\right] \\
& A_{33}=-\frac{1}{4 \alpha^{2}}\left[\frac{25}{\alpha^{4}}+\frac{65}{3 \alpha^{2}}+4-\left(\frac{25}{\alpha^{4}}+\frac{30}{\alpha^{2}}+9\right) \beta\right]
\end{aligned}
$$

Evaluation of Equation (11) with $\mathrm{B}=\mathrm{iC}$ results in

$$
\begin{aligned}
& A_{00}=\beta=\frac{\tanh ^{-1} \alpha}{\alpha} \\
& A_{01}=\frac{1-\beta}{\alpha} \\
& A_{02}=-\frac{3}{2} \frac{(1-\beta)}{\alpha^{2}}-\frac{\beta}{2} \\
& A_{11}=-\frac{1-\beta}{\alpha^{2}} \\
& A_{12}=\frac{3}{2} \frac{(1-\beta)}{\alpha^{3}}+\frac{\beta}{2 \alpha} \\
& A_{22}=\frac{1}{4 \alpha}\left[\left(\frac{9}{\alpha^{3}}-\frac{6}{\alpha}+\alpha\right) \beta+\frac{3}{\alpha}-\frac{9}{\alpha^{3}}\right] \\
& A_{03}=-\frac{1}{2 \alpha}\left[\left(\frac{5}{\alpha^{2}}-3\right) \beta+\frac{4}{3}-\frac{5}{\alpha^{2}}\right] \\
& A_{13}=\frac{1}{2 \alpha^{2}}\left[\left(\frac{5}{\alpha^{2}}-3\right) \beta+\frac{4}{3}-\frac{5}{\alpha^{2}}\right] \\
& A_{23}=\frac{1}{4 \alpha}\left[\frac{15}{\alpha^{4}}-\frac{9}{\alpha^{2}}+\frac{4}{3}-\left(\frac{15}{\alpha^{4}}-\frac{14}{\alpha^{2}}+3\right) \beta\right] \\
& A_{33}=-\frac{1}{4 \alpha^{2}}\left[\frac{25}{\alpha^{4}}-\frac{65}{3 \alpha^{2}}+4-\left(\frac{25}{\alpha^{4}}-\frac{30}{\alpha^{2}}+9\right) \beta\right]
\end{aligned}
$$


Evaluation of Equation (11) with $\mathrm{B}=-\mathrm{iC}$ results in

$$
\begin{aligned}
& A_{00}=\beta=\frac{\tanh ^{-1} \alpha}{\alpha} \\
& A_{01}=\frac{\beta-1}{\alpha} \\
& A_{02}=-\frac{3}{2} \frac{(1-\beta)}{\alpha^{2}}-\frac{\beta}{2} \\
& A_{11}=\frac{\beta-1}{\alpha^{2}} \\
& A_{12}=\frac{3}{2} \frac{(\beta-1)}{\alpha^{3}}-\frac{\beta}{2 \alpha} \\
& A_{22}=\frac{1}{4 \alpha}\left[\left(\frac{9}{\alpha^{3}}-\frac{6}{\alpha}+\alpha\right) \beta+\frac{3}{\alpha}-\frac{9}{\alpha^{3}}\right] \\
& A_{03}=-\frac{1}{2 \alpha}\left[\left(\frac{5}{\alpha^{2}}-3\right) \beta-\frac{4}{3}+\frac{5}{\alpha^{2}}\right] \\
& A_{13}=\frac{1}{2 \alpha^{2}}\left[\left(\frac{5}{\alpha^{2}}-3\right) \beta+\frac{4}{3}-\frac{5}{\alpha^{2}}\right] \\
& A_{23}=\frac{1}{4 \alpha}\left[-\frac{15}{\alpha^{4}}+\frac{9}{\alpha^{2}}-\frac{4}{3}+\left(\frac{15}{\alpha^{4}}-\frac{14}{\alpha^{2}}+3\right) \beta\right] \\
& A_{33}=-\frac{1}{4 \alpha^{2}}\left[\frac{25}{\alpha^{4}}-\frac{65}{3 \alpha^{2}}+4-\left(\frac{25}{\alpha^{4}}-\frac{30}{\alpha^{2}}+9\right) \beta\right]
\end{aligned}
$$

Thus, using the coefficients defined by Equation (17), (18), and (19), Equation (7) defines the $B_{3}$ fluxes, which are all real. When $B$ is real, the spatial distribution is of $\operatorname{cosine}(B \mathrm{x})$, where $B^{2}$ is the geometric buckling of the fundamental mode of the critical homogeneous geometry. When $B=\mathrm{i} C$ with a real constant $C$, the spatial distribution is of positive exponential form with the negative buckling. In this case, there is a neutron leakage into the region, i.e., a negative leakage, which might be useful under certain circumstances. When $B=-\mathrm{i} C$ with a real constant $C$, the spatial distribution is of negative exponential form with the negative buckling and the solution would behave like the case with the positive buckling. In the COMBINE7.1 coding, the series expansions of $\tan ^{-1} \alpha$ and $\tanh ^{-1} \alpha$ are utilized to calculate these coefficients. The fundamental limitation of the cases with the negative buckling is in the fact that solutions involve a hyperbolic tangent, $\tanh ^{-1}\left(B / \Sigma_{t}\right)$. As can be seen in

$$
\tanh ^{-1}\left(B / \Sigma_{t}\right)=\frac{1}{2} \log \left[\left(1+B / \Sigma_{t}\right) /\left(1-B / \Sigma_{t}\right)\right]
$$

$B / \Sigma_{t}$ should be less than 1 . Normally, $B / \Sigma_{t}$ is much less than 1 ; however, it can be reset to a value less than $1(0.8$, e.g.) to avoid divergence when it is large.

The energy dependence of Equation (7) is approximated by a 167 fine group representation, spanning the energy range from $20 \mathrm{MeV}$ to $1.0 \mathrm{E}-5 \mathrm{eV}$, for solution of the neutron spectrum. Integrating Equation (7) over the group energy interval, i.e., $E_{g} \leq E \leq E_{g-1}$ and truncating to $\mathrm{L}+1$ terms generates 


$$
\begin{aligned}
& \phi_{n}^{g} \\
& =\sum_{l=0}^{L}(2 l+1) \frac{A_{n l}^{g}}{\Sigma_{t}^{g}} \sum_{g^{\prime}=1}^{G} \Sigma_{s l}^{g^{\prime} \rightarrow g} \phi_{l}^{g^{\prime}} \frac{\Delta g^{\prime}}{\Delta g}+\frac{A_{n 0}^{g}}{\sum_{t}^{g}} \frac{\chi^{g}}{\Delta_{g}}
\end{aligned}
$$

where

$$
\begin{aligned}
& \phi_{n}^{g}=\int_{E_{g}}^{E_{g-1}} \phi_{n}(E) \frac{d E}{E} / \Delta_{g} \\
& \Delta_{g}=\int_{E_{g}}^{E_{g-1}} \frac{d E}{E}
\end{aligned}
$$

The maximum group number is designated as $\mathrm{G}$ in Equation (20). It is noted that the fluxes are now expressed as per-unit energy in lethargy coordinates. Equation (20) includes neutrons scattered down from all higher energy groups. It also includes neutrons scattered up from all lower energy groups if thermal upscattering occurs.

The $B_{3}$ solution to Equation (20) is given by the following matrix equation for each energy group g.

$$
\begin{aligned}
& {\left[\mathrm{I}-C_{g}\right] \Phi_{g}=R_{g}} \\
& \Phi_{g}=\left[\Phi_{0}^{g}, \Phi_{1}^{g}, \Phi_{2}^{g}, \Phi_{3}^{g}\right] . \\
& C_{n l}^{g}=(2 l+1) \frac{A_{n l}^{g}}{\sum_{t}^{g}} \sum_{s l}^{g \rightarrow g}
\end{aligned}
$$

where $\mathrm{I}$ is the four-rowed unit matrix, $\mathrm{C}_{\mathrm{g}}$ is the matrix with 4 rows and 4 columns, and $n, l$ are the row and column index, respectively, for $C_{g}$ in Equation (21).

$$
\begin{aligned}
& R_{n}^{g}=\frac{A_{n 0}^{g}}{\sum_{t}^{g}} \frac{\chi^{g}}{\Delta g}+\sum_{l=0}^{3}(2 l+1) \frac{A_{n l}^{g}}{\sum_{t}^{g}} \sum_{g^{\prime}=1, g^{\prime} \neq g}^{G} \sum_{s l}^{g^{\prime} \rightarrow g} \Phi_{l}^{g^{\prime}} \frac{\Delta g^{\prime}}{\Delta g} \\
& R_{g}=\left[R_{0}^{g}, R_{1}^{g}, R_{2}^{g}, R_{3}^{g}\right] .
\end{aligned}
$$

When there is no thermal upscattering, Equation (21) is solved by Gauss elimination, sweeping from the highest energy to the lowest energy. When the thermal upscattering is present, it is solved by the combination of Gauss elimination and Gauss-Seidel iterative scheme. In this case, the thermal upscattering terms will be included, initially using assumed flux guess.

The fission source, $\chi$, can be chosen from a fissile/fissionable isotope or calculated from the steady-state and fission rate weighted source spectrum. The steady-state fission spectrum for an isotope, $i$, is defined by

$$
\chi_{i, g}^{S S}=\frac{\sum_{g^{\prime}} \sigma_{f, g^{\prime} \rightarrow g} \Phi_{g^{\prime}}+\chi_{g}^{D} \sum_{g^{\prime}} v_{g^{\prime}}^{D} \sigma_{f, g^{\prime}} \Phi_{g^{\prime}}}{\sum_{g} \sum_{g^{\prime}} \sigma_{f, g^{\prime} \rightarrow g} \Phi_{g^{\prime}}+\sum_{g} \chi_{g}^{D} \sum_{g^{\prime}} v_{g^{\prime}}^{D} \sigma_{f, g^{\prime}} \Phi_{g^{\prime}}} .
$$

The fission rate weighted spectrum is then 
$\chi_{g}=\frac{\sum_{i} \chi_{i, g}^{S S} \sum_{g^{\prime}} v_{t o t, g^{\prime}}^{i} N_{i} \sigma_{f, g}^{i} \Phi_{g^{\prime}}}{\sum_{i} \sum_{g^{\prime}} v_{t o t, g^{\prime}}^{i} N_{i} \sigma_{f, g^{\prime}}^{i} \Phi_{g^{\prime}}}$.

The flux as defined in Equation (41) and included in the cross section library is initially used in Equation (27) and then the $B_{N}$ spectrum calculated flux when once more spectrum calculation is performed.

\subsubsection{B-1 Approximation}

The $B_{1}$ equations can be the degenerate forms of $B_{3}$. Instead, $B_{1}$ equations with real $\mathrm{B}$ for instance are derived from Equation (7), emphasizing the physical approximations

$$
\begin{aligned}
& \phi_{0}(B, E) \\
& =\frac{\beta}{\Sigma_{t}(E)} \int T_{S 0}\left(E^{\prime} \rightarrow E\right) \phi_{0}(B, E) d E^{\prime}+3 \frac{(\beta-1)}{\alpha \Sigma_{t}} \int T_{S 1}\left(E^{\prime} \rightarrow E\right) \phi_{1}(B, E) d E^{\prime}+\frac{\beta}{\Sigma_{t}} \chi(E) \\
& \phi_{1}(B, E) \\
& =-\frac{(\beta-1)}{\alpha \Sigma_{t}(E)} \int \Sigma_{S 0}\left(E^{\prime} \rightarrow E\right) \phi_{0}(B, E) d E^{\prime}+3 \frac{(1-\beta)}{\alpha^{2} \Sigma_{t}(E)} \int \Sigma_{S 1}\left(E^{\prime} \rightarrow E\right) \phi_{1}(B, E) d E^{\prime}-\frac{(\beta-1)}{\alpha \Sigma_{t}(E)} \chi(E)
\end{aligned}
$$

Solve Equations (28) and (29) for $\phi_{0}$ and $\phi_{1}$ to obtain

$$
\begin{aligned}
& B \Phi_{1}+\Sigma_{t} \Phi_{0}=\int_{E^{\prime}} \Sigma_{S O}\left(E^{\prime} \rightarrow E\right) \Phi_{0}\left(E^{\prime}\right) d E^{\prime}+\chi(E), \\
& \gamma(E) \Sigma_{t}(E) \Phi_{1}(E)-\frac{B}{3} \Phi_{0}(E)=\int_{E^{\prime}} \Sigma_{s 1}\left(E^{\prime} \rightarrow E\right) \Phi_{1}\left(E^{\prime}\right) d E^{\prime}
\end{aligned}
$$

where

$\gamma(E)=\frac{1}{3} \frac{\beta \alpha^{2}}{(1-\beta)}$.

$\Phi_{0}(E)$ and $\Phi_{1}(E)$ are interpreted as the energy-dependent flux and current, respectively. By

examining Equations (30) and (31), it is seen that the $B_{1}$ approximation differs from the $P_{1}$ approximation by the factor $\gamma(\mathrm{E})$ in Equation (31). If $\gamma(\mathrm{E})$ is set to equal to 1.0, Equations (30) and (31) become the continuity equations for the $P_{1}$ approximation. The factor $\gamma(\mathrm{E})$ tends to improve the $B_{1}$ estimation of the energy-dependent transport current, $\Phi_{1}(E)$, for small homogeneous system. ${ }^{16}$

Similarly, for $\mathrm{B}=-\mathrm{iC}$

$$
\begin{aligned}
& -C \Phi_{1}+\Sigma_{t} \Phi_{0}=\int_{E^{\prime}} \Sigma_{S O}\left(E^{\prime} \rightarrow E\right) \Phi_{0}\left(E^{\prime}\right) d E^{\prime}+\chi(E), \\
& -\gamma(E) \Sigma_{t}(E) \Phi_{1}(E)-\frac{C}{3} \Phi_{0}(E)=\int_{E^{\prime}} \Sigma_{s 1}\left(E^{\prime} \rightarrow E\right) \Phi_{1}\left(E^{\prime}\right) d E^{\prime}
\end{aligned}
$$

For $\mathrm{B}=\mathrm{iC}$

$$
C \Phi_{1}+\Sigma_{t} \Phi_{0}=\int_{E^{\prime}} \Sigma_{S O}\left(E^{\prime} \rightarrow E\right) \Phi_{0}\left(E^{\prime}\right) d E^{\prime}+\chi(E),
$$


$-\gamma(E) \Sigma_{t}(E) \Phi_{1}(E)+\frac{C}{3} \Phi_{0}(E)=\int_{E^{\prime}} \Sigma_{S 1}\left(E^{\prime} \rightarrow E\right) \Phi_{1}\left(E^{\prime}\right) d E^{\prime}$

Equations (30) and (31) in multigroup from reduce to

$B \Phi_{1}^{g}+\sum_{t}^{g} \Phi_{0}^{g}=\sum_{g^{\prime}=1}^{G} \sum_{s 0}^{g^{\prime} \rightarrow g} \Phi_{0}^{g^{\prime}} \frac{\Delta g^{\prime}}{\Delta g}+\frac{\chi^{g}}{\Delta g}$

and

$\gamma^{g} \sum_{t}^{g} \Phi_{1}^{g}-\frac{B}{3} \Phi_{0}^{g}=\sum_{g^{\prime}=1}^{G} \sum_{s 1}^{g^{\prime} \rightarrow g} \Phi_{1}^{g} \frac{\Delta g^{\prime}}{\Delta g}$.

Equations similar to (36) and (37) are derived for imaginary B from Equations (32) through (35) with variations of sign. These equations are solved simultaneously as

$\Phi_{0}^{g}=\frac{R_{0}^{g}\left(\gamma^{g} \sum_{t}^{g}-\Sigma_{s 1}^{g \rightarrow g}\right)-B R_{1}^{g}}{\left(\sum_{t}^{g}-\Sigma_{s 0}^{g \rightarrow g}\right)\left(\gamma^{g} \sum_{t}^{g}-\Sigma_{s 1}^{g \rightarrow g}\right)+\frac{B^{2}}{3}}$

$\Phi_{1}^{g}=\frac{R_{1}^{g}+\frac{B}{3} \Phi_{0}^{g}}{\gamma^{g} \sum_{t}^{g}-\Sigma_{s 1}^{g \rightarrow g}}$

where

$$
R_{0}^{g}=\sum_{g^{\prime}=1, g^{\prime} \neq g}^{G} \sum_{s 0}^{g^{\prime} \rightarrow g} \Phi_{0}^{g^{\prime}} \frac{\Delta g^{\prime}}{\Delta g}+\frac{\chi^{g}}{\Delta g} \text { and } R_{1}^{g}=\sum_{g^{\prime}=1, g^{\prime} \neq g}^{G} \sum_{s 1}^{g^{\prime} \rightarrow g} \Phi_{1}^{g^{\prime}} \frac{\Delta g^{\prime}}{\Delta g}
$$

When upscattering is present, Gauss-Seidel iterative scheme is used.

\subsubsection{Convergence on Gauss-Seidel Iterative Scheme}

The calculation will be converged when the following equations are satisfied at all energy points.

$\frac{\left|\phi_{i}^{(m+1)}-\phi_{i}^{(m)}\right|}{\phi_{i}^{(m+1)}}<\varepsilon$ and $\frac{\left|J_{i}^{(m+1)}-J_{i}^{(m)}\right|}{J_{i}^{(m+1)}}<\varepsilon$,

where $\varepsilon$ is the user input convergence criteria. The results are then normalized to have one total scalar flux.

\subsection{Bondarenko Method}

The multigroup approximation requires the groupwise cross sections and the energy texture is necessarily coarse with respect to the resonance structure in many materials. The resonance results in selfshielding effect. The magnitude of this self-shielding effect is in general a complicated function of the geometry and composition of the system. However, it has been found that a simple model called the Bondarenko Method ${ }^{8}$ or Background Cross Section Method ${ }^{17}$ does a surprisingly good job of representing the effects for many applications. ${ }^{9}$ This method was adopted in the NJOY's GROUPR and MATXSR modules and the TRANSX code. ${ }^{10}$ COMBINE7.1 followed those steps taken in the NJOY and TRANSX codes. The flux is assumed to vary inversely as the total macroscopic cross section. In the Bondarenko model, the narrow resonance (NR) approximation and the $B_{\mathrm{N}}$ approximation for large systems are invoked. ${ }^{9}$ The model flux for the group averages for isotope $i$ is written as 
$\Phi_{l}^{i}(E)=\frac{C(E)}{\left[\sigma_{0}^{i}+\sigma_{t}^{i}(E)\right]^{l+1}}$

where $C(E)$ is the smooth part of the shape of the flux, $\sigma_{i}^{i}(E)$ is the microscopic total cross section for isotope $\mathrm{i}$, and $\sigma_{0}^{i}$ is called the background cross section (it represents the effects of all the other isotopes). The effect of the total cross section in the denominator is to put a dip in the flux for each peak in the cross section, and $\sigma_{0}^{i}$ controls the relative size of the dip. A resonance material in a dilute mixture or in small pieces does not disturb a smooth flux very much by it presence - this is called the infinitely dilute case. When $\sigma_{0}^{i}$ approaches a very large number, it becomes an infinitely dilute case. The $l$ dependence shown here is appropriate for a large system with nearly isotropic scattering (the $B_{0}$ approximation), and it was used when the MATXS file was generated from NJOY.

The multigroup form of this model flux is

$\Phi_{\mathrm{lg}}^{i}=\frac{C_{g}}{\left[\sigma_{0, g}^{i}+\sigma_{t, g}^{i}\right]^{l+1}}$.

The resonance absorption changes with the temperature due to Doppler broadening of the resonances. In order to take into account the effect of Doppler broadening, the basic cross sections in the resonance region must be adjusted appropriately for the broadening before they are used in computing group constants.

The Bondarenko method is basically an "infinite medium" method that parameterizes cross sections for a nuclide as a function of temperature, $\mathrm{T}$, and the "background dilution" cross section, $\sigma_{0}$, of all other nuclides mixed with the nuclide. Simplistically, given the temperature and background dilution cross-section values, one determines self-shielded cross sections by interpolating in tables. Since self-shielding causes the "background dilution" values that a nuclide sets to change, an iterative procedure involving all nuclides is used. The geometric lump effect when present is accounted for by augmenting the background cross section.

For a homogeneous mixture, the appropriate background cross section is

$\sigma_{0, g}^{i}=\frac{1}{N_{i}} \cdot \sum_{j \neq i} N_{j} \cdot \sigma_{t, g}^{j}\left(\sigma_{0, g}^{i}\right)$

Where $\mathrm{N}$ is the number density for the isotope and $\sigma_{t, g}$ is the self-shielded total cross section of an energy group g. Because $\sigma_{t, g}$ depends on $\sigma_{0, g}$, finding $\sigma_{0, g}$ is an iterative process. For a mixture of resonance materials, interference between resonances in different materials is handled in an average sense only.

For a lump of resonance material embedded in a large moderating region, escapes from the lump also increase the background cross section. To account for this, the $\sigma_{0, g}$ value is augmented by an escape cross section

$\sigma_{e, g}^{i}=\frac{1}{N_{i} \bar{l}}$

where $\bar{l}$ is the mean chord length of lump given by

$\bar{l}=\frac{4 V}{S}$ 
and where $V$ and $S$ are the volume and surface area of the lump. Multizone situations, such as reactor lattices, are accounted for by the use of Dancoff factors, which, in effect, modify the escape probability and, hence, the value of $\sigma_{e}$. The mean chord length $\bar{l}$ can be adjusted away from the geometric value of Equation (44) to compensate for the presence of other lumps (Dancoff correction) or for shortcoming in the escape probability model used to obtain Eq. (43). In the rational approximation, the Dancoff correction is equivalent to increasing the mean chord length, $\bar{l}$, by the factor $1 /(1-\mathrm{c})$ or equivallently decreasing the surface area of lump by 1-c as in

$$
\sigma_{e, g}^{i}=\frac{1}{N_{i} \cdot \bar{l} \cdot \frac{1}{(1-c)}}
$$

when Bell corrections are applied

$$
\sigma_{e, g}^{i}=\frac{1}{N_{i} \cdot \bar{l}} \cdot \frac{b_{1} \cdot(1-c)}{1+\left(b_{2}-1\right) \cdot c}
$$

where $\mathrm{b} 1$ and $\mathrm{b} 2$ are called Bell corrections. When $\mathrm{b} 1=\mathrm{b} 2$, the constant is usually called the Levine factor and Equation (46) becomes

$$
\sigma_{e, g}^{i}=\frac{1}{N_{i} \cdot l} \cdot \frac{1}{\frac{1}{b}+\frac{c}{1-c}}
$$

where

$$
\begin{aligned}
b & =1.26 \text { for the slab geometry } \\
& =1.35 \text { for the cylindrical geometry } \\
& =1.30 \text { for the spherical geometry. }
\end{aligned}
$$

The so-called "Levine factors," $b$, are approximate geometry dependent factors that are approximately correct for inaccuracies in the Rational Approximation.

When a double heterogeneity occurs due to fuel grains present within the macroscopic fuel lump, the Dancoff factor c' specifies the mutual shielding between these grains, independent of $\mathrm{c}$ for the lumps themselves. To better account for the double heterogeneity due to fuel grains present within the macroscopic fuel lump, a correction has been made to the escape probability and given by Stamatelatos and LaBauve ${ }^{18}$ as

$$
\sigma_{e, g}^{i}=\frac{1}{N_{i}\left[\bar{l} \cdot\left(\frac{1}{b}+\frac{c}{1-c}\right)+\bar{l}_{G}\left(\frac{1}{a}+\frac{c^{\prime}}{1-c^{\prime}}\right)\right]}
$$

where

$\bar{l}_{G}=$ the average chord length of a single isolated spherical fuel grain

$c^{\prime}=$ the Dancoff factor for fuel grains

A $=1.77$, the Levine factor for spherical grains. 
The following equation for the background cross section has been applied

$\sigma_{0, g}^{i}=\sigma_{e, g}^{i}+\frac{\sum_{i \neq j} N_{j} \cdot \sigma_{t, g}^{j}\left(\sigma_{0, g}^{j}\right)}{N_{i}}$.

\subsubsection{Fine-Group Cross Section Generation in Bondarenko Format}

In COMBINE7.1, a 167 fine group structure is used and the multigroup cross section constants are generated in this fine group structure to be used in the $B_{\mathrm{N}}$ solution.

The NJOY99.259 version ${ }^{15}$ was used to generate a 167 fine-group cross-section library, MATXS.LIB, in MATXS format (Bondarenko format) on sets of background cross sections and temperatures including thermal scattering cross sections. For C(E) in Equation (40), an NJOY built-in spectrum, Thermal--1/E-Fast Reactor--Fission + Fusion, is used. The equations providing the fundamental definitions in NJOY for the multigroup cross sections and the group-to-group matrix are

$$
\begin{aligned}
& \sigma_{t, l, g}=\frac{\int_{g} \sigma_{t}(E) \phi_{l}(E) d E}{\int_{g} \phi_{l}(E) d E}, \\
& \sigma_{x, g}=\frac{\int_{g} \sigma_{x}(E) \phi_{0}(E) d E}{\int_{g} \phi_{0}(E) d E}, \\
& \sigma_{X, l, g^{\prime} \rightarrow g}=\frac{\int_{g} d E \int_{g^{\prime}} d E^{\prime} \sigma_{X l}\left(E^{\prime} \rightarrow E\right) \phi_{l}(E)}{\int_{g} \phi_{l}(E) d E} .
\end{aligned}
$$

The energy-dependent cross-section database is now derived entirely from ENDF/B-VII.0 nuclear data with the exception of $\mathrm{Zr}$ element cross-section data, which is based on ENF/B-VI.8 nuclear data. All materials have either $S(\alpha, \beta)$ or free thermal scattering matrices.

\subsubsection{Bondarenko Self-Shielding Interpolation}

Bondarenko self-shielding is performed in COMBINE7.1 by background cross section and temperature interpolation. The interpolation is first performed for the background cross sections at each temperature. The generated cross sections for the set of preselected temperatures are then interpolated for the problem-dependent temperature. In the background cross-section interpolation, a $\log -\log \mathrm{N}^{\text {th }}$ order for the vector cross sections, and log-linear $\mathrm{N}^{\text {th }}$ order Lagrangean schemes are employed with a variation to the first order for the extremely high or low problem-dependent background cross section. The temperature interpolation uses a $\log -\log \mathrm{N}^{\text {th }}$ order Lagrangean scheme. Iteration is necessary to arrive at the correct background cross sections. Three iterations are performed for all isotopes. The first iteration is based on the infinite dilution of the rest isotopes for total cross sections. The accuracy, convergence, and stability of these schemes have been sufficiently tested and are satisfactory. 


\subsection{Resonance Region}

The material cross-section libraries supplied for each material have complete multigroup cross sections based on Bondarenko method. The weakness of the Bondarenko model occurs for thermal reactor analysis in the "epithermal" energy region from about $4 \mathrm{eV}$ to around 200 or $300 \mathrm{eV} .{ }^{10} \mathrm{In}$ this region, the resonances are no longer narrow, and the flux shape given by Equation (40) is no longer sufficiently accurate. For those materials where resolved resonance parameters are present via RERES.LIB, a Nordheim numerical solution ${ }^{11}$ is applied as an option. Before $B_{1}$ or $B_{3}$ Equations are solved, the resonance parameters in the material libraries must be converted into average, self-shielded, temperature dependent, groupwise cross sections.

\subsubsection{Resolved Resonances-Nordheim Numerical Method}

The balance equation for the collision density in a homogeneous mixture of moderators and resonance absorbers in an infinite medium is ${ }^{19}$

$$
F(E)=\phi(E) \sum_{t}(E)=\sum_{k} \int_{E}^{E / \alpha_{k}} \frac{\phi\left(E^{\prime}\right) \sum_{s}^{k}\left(E^{\prime}\right) d E^{\prime}}{\left(1-\alpha_{k}\right) E^{\prime}}
$$

where

$$
\begin{aligned}
& \phi(E)=\text { scalar neutron flux } \\
& \Sigma_{\mathrm{t}}=\text { total macroscopic cross section } \\
& \sum_{s}^{k} \quad=\mathrm{N}_{\mathrm{k}} \sigma_{\mathrm{sk}}=\text { macroscopic scattering cross section for material } \mathrm{k} \\
& \mathrm{N}_{\mathrm{k}} \quad=\text { atomic density of material } \mathrm{k} \\
& \sigma_{\mathrm{k}} \quad=\text { microscopic cross section of material } \mathrm{k} \\
& \alpha_{k} \quad=\left(\frac{A_{k}-1}{A_{k}+1}\right)^{2}, \mathrm{~A}_{\mathrm{k}}=\text { ratio of isotopic mass to mass of the neutron for material } \mathrm{k} \\
& \mathrm{F}(\mathrm{E}) \quad=\text { collision density at energy E. }
\end{aligned}
$$

The right-hand side of Equation (53) requires a different integration limit for each moderator $\mathrm{k}$. The flux $\phi(E)$ at each resolved resonance undergoes a severe depression at each strongly absorbing resonance. One or many resonances may fall within any given COMBINE multigroup. The average, selfshielded, absorption integral in each multigroup $\mathrm{g}$ within the resonance range is then given by

$$
I_{a g}^{k}=\int_{E g}^{E_{g+1}} \phi(E) \sigma_{c}^{k}(E) d E
$$

where $\phi(E)$ is the solution of Equation (53) over the energy interval for the multigroup and $\sigma_{c}$ is the capture cross section. Equation (53) is solved in lethargy space where

$$
\begin{array}{ll}
\mathrm{du} & =-\frac{d E}{E} \\
\mathrm{dE} & =-\mathrm{Edu} \\
\mathrm{E} & =\mathrm{E}_{1} \mathrm{e}^{-\mathrm{u}} .
\end{array}
$$

Therefore, $d E=E_{1} e^{-u} d u$.

Equation (55) is transformed to ${ }^{11}$ 


$$
\begin{aligned}
& I_{c g}^{k}=E_{1} \int_{u_{g-1}}^{u_{g}} \phi[E(u)] \sigma_{c}^{k}[E(u)] e^{-u} d u \\
& I_{c g}^{k}=E_{1} \int_{u_{g-1}}^{u_{g}} F[u(E)] \frac{\sigma_{c}^{k}(u) e^{-u}}{\sum_{t}(u)} d u
\end{aligned}
$$

where $\mathrm{F}[\mathrm{u}(\mathrm{E})]$ is the collision density per unit energy in lethargy coordinates. The mixed lethargy-energy form of Equation (55) results from the fact that the integration is performed on a lethargy mesh, but the resonance cross sections are calculated in energy space because the cross sections are not easily transformed to lethargy coordinates. Equations similar to Equation (55) may be written for the fission and elastic scattering integrals using $\sigma_{f}^{k}(u)$ or $\sigma_{s}^{k}(u)$ in place of $\sigma_{c}^{k}(u)$.

If absorption occurs in an isolated lump surrounded by an infinite sea of moderators, the introduction of the lump escape probability ${ }^{20} P_{0}\left(\bar{l}, \Sigma_{t}\right)$, is given in terms of the average chord length of the lump, $\bar{l}$. This enables Equation (53) to be modified to closely approximate the heterogeneous absorption rate in the lump-moderator assembly. The collision density in the lump now consists of two distinct types of events:

1. Those neutrons that have had their last collision in the lump, which is given by the right-hand side of Equation (53) multiplied by the probability of colliding in the lump, $\left[1-P_{0}\left(\bar{l}, \Sigma_{t}\right)\right]$, times $\mathrm{V}_{\mathrm{F}}$, the volume of the fuel lump.

2. Those neutrons that stream into the lump at lethargy $\mathrm{u}(\mathrm{E})$ from outside the lump, multiplied by the moderator escape probability ${ }^{20} P_{M}\left(\bar{l}, \Sigma_{t}\right)$, times the volume of the external moderator, $\mathrm{V}_{\mathrm{M}}$, contained in a unit cell.

$V_{F} F(u)=V_{F}\left[1-P_{0}\left(\bar{l}, \Sigma_{t}\right)\right] \sum_{k} \frac{1}{1-\alpha_{k}} \int_{u-\varepsilon_{k}}^{u} F\left(u^{\prime}\right) \frac{\sum_{S}^{k}\left(u^{\prime}\right)}{\sum_{t}\left(u^{\prime}\right)} d u^{\prime}+V_{M} P_{M} \phi_{M}(u) \sum_{M}(u)$

where

$\varepsilon_{k}=\ln \left(\frac{1}{\alpha_{k}}\right)$

and $\phi_{M}(u)$ is the average, asymptotic, equilibrium neutron flux in the moderator residing outside the lump. The normalization of Equation (56) is arbitrary, since the point wise collision density $\mathrm{F}(\mathrm{u})$ will only be used to obtain average cross sections over the multigroups in the resolved resonance range. $\phi[u(E)]$ is set equal to $1 / E$ in the region outside the lump, i.e., $\phi_{\text {asym }}[u(E)]=e^{u} / E_{1}$.

The moderator terms in Equation (56) may be removed by means of the reciprocity relation ${ }^{9}$ $P_{M} V_{M} \Sigma_{M}=P_{F} V_{F} \Sigma_{t F}$

Equation (56) then becomes

$$
F(u)=\left(1-P_{0}\right) \sum_{k-1}^{7} \frac{1}{1-\alpha_{k}} \int_{u-\varepsilon_{k}}^{u} F\left(u^{\prime}\right) \frac{\sigma_{s}^{k}\left(u^{\prime}\right)}{\sigma_{t}\left(u^{\prime}\right)} d u^{\prime}+P_{0} \sigma_{t}(u) \frac{e^{u}}{E_{1}}
$$

where $\sigma_{s}^{k}$ and $\sigma_{t}$ are given by Equations (62) and (63), expressed in terms of the scattering per absorber atom. 


$$
P_{0}=P_{0}\left(\bar{l}, \sum_{t}\right)
$$

where

$$
\begin{aligned}
\bar{l} & =\bar{l}_{0}=\frac{4 V}{S}, \text { single isolated lump } \\
\frac{V}{S} & =\text { ratio of volume of the absorber lump to the surface area } \\
& =1 / 2 \times \text { half thickness - plate geometry } \\
& =1 / 2 \times \text { radius - cylindrical geometry } \\
& =1 / 3 \times \text { radius - spherical geometry }
\end{aligned}
$$

The escape probability $\mathrm{P}_{0}$ is tabulated ${ }^{11}$ for single isolated lumps. When a Dancoff factor $\mathrm{C}$ is incorporated to represent the mutual shielding in a repetitive array, ${ }^{21}$ the escape probability $\mathrm{P}_{0}$ is modified to $\mathrm{be}^{11,22}$

$$
P=\frac{G P_{0}(1-C)}{\left[1-\left(1-\bar{l} \Sigma_{t} P_{0}\right) C\right]}
$$

where $\mathrm{P}_{0}$ in Equation (60) is the escape probability for a single isolated lump, P represents the mutually shielded value, $\mathrm{C}$ is the Dancoff Ginsberg correction factor for a regular array of lumps, and $\mathrm{G}$ is a double heterogeneity correction for the self-shielding of fuel grains within the absorber lump ${ }^{23}$

$$
G=\left[1+\sum_{t} \bar{l}_{G}\left(\frac{1}{a}+\frac{C^{\prime}}{1-C^{\prime}}\right)\right]^{-1}
$$

where

$\bar{l}_{G}=$ average chord length within a fuel grain, independent of $\bar{l}$, the average chord length in the lump

$C^{\prime}=$ Dancoff factor for the fuel grains in the lump, independent of $\mathrm{C}$

$\mathrm{a}=1.77$ - the Levine factor for spherical grains, A correction factor for the Wigner Rational Approximation for $\mathrm{P}_{0}$, which is employed in deriving Equation (62).

When a double heterogeneity occurs due to fuel grains present within the macroscopic fuel lump, the Dancoff factor $\mathrm{C}^{\prime}$ specifies the mutual shielding between these grains, independent of $\mathrm{C}$ for the lumps themselves.

In Equation (58), up to seven moderators are included for the down scatter source within the lump, the first always being the absorber atom itself. Three additional moderators and three additional admixed resonance absorbers with different masses can also be included. ${ }^{3}$ This limit is felt to be sufficient for almost any fuel pin one would encounter in practice.

The cross sections in Equation (58) are evaluated relative to the number of absorber atoms as in 
$\sigma_{s}^{k}=\frac{\sum_{s}^{k}}{N_{a}}$

where

$\mathrm{N}_{\mathrm{a}}=$ atom density of the absorber atom in the lump.

$\sigma_{t}=\sigma_{a}+\sigma_{s}+\sigma_{m}^{1}+\sigma_{m}^{2}+\sigma_{m}^{3}+\sigma_{a}^{1}+\sigma_{s}^{1}+\sigma_{a}^{2}+\sigma_{s}^{2}+\sigma_{a}^{3}+\sigma_{s}^{3}$

where: $\sigma_{s}$ is the resonance scattering of the absorber atom itself; $\sigma_{m}^{i}$ represents the three moderator's scattering cross sections per absorber atom; $\sigma_{a}^{i}$ represents the three admixed absorber's absorption cross sections per absorber atom; and $\sigma_{s}^{i}$ represents the three admixed absorber's scattering cross sections per absorber atom. The absorption cross section of the three additional moderators is assumed to be very small compared with the principal absorber or the admixed absorbers.

Equation (58) has proven itself to be remarkably accurate in many applications. A number of unique approximations are used in deriving Equation (58), the most important of which is the "flat flux assumption." 24 The spatial dependence of the flux across the lump has been subsumed into the escape probability $\mathrm{P}_{0}$, which permits the elimination of the spatial dependence of the flux in Equation (58). $\mathrm{P}_{0}$ is evaluated as a function of energy only. The approximation which makes this possible is that at each energy, the escape probability is evaluated as if only the average flat flux in the lump were known relative to the asymptotic flat flux existing outside the lump. The escape probability $\mathrm{P}_{0}$ under the flat flux approximation is then given by ${ }^{24}$

$$
P_{0} \approx \frac{\bar{\phi}_{\text {lump }}}{\bar{\phi}_{\text {asym }}}
$$

If an average flat flux can be assumed in the lump, the escape probability absorbs the spatial dependence in Equation (58). $P_{0}\left(\bar{l}, \sum_{t}\right)$ is evaluated at each energy across the resonance since $\sum_{\mathrm{t}}(\mathrm{E})$ is a strong function of energy at resonance. Equation (64) is a truly remarkable result that makes possible a separate two-stage solution to a difficult problem; the first stage being the calculation of the escape probability $\mathrm{P}_{0} .{ }^{11}$ Equation (58) is solved numerically on a very fine energy mesh across each lethargy group where one or more resonances may reside.

\subsubsection{Doppler Broadening of Resolved Resonance Parameters}

Subroutines adopted from the NJOY code and incorporated in COMBINE7.1 numerically Doppler broaden the SLBW, MLBW, and R-M format resonance parameters by the kernel broadening method. ${ }^{25}$ The subroutines were verified by kernel broadening the SLBW option and comparing with that using the $\psi-\chi$ function that was used in the previous COMBINE versions. At each mesh point the capture, fission, and elastic cross sections are calculated, accumulating contributions from all the resonances in the same resonance range. Figure 2 shows the Doppler broadened cross section as an example. 


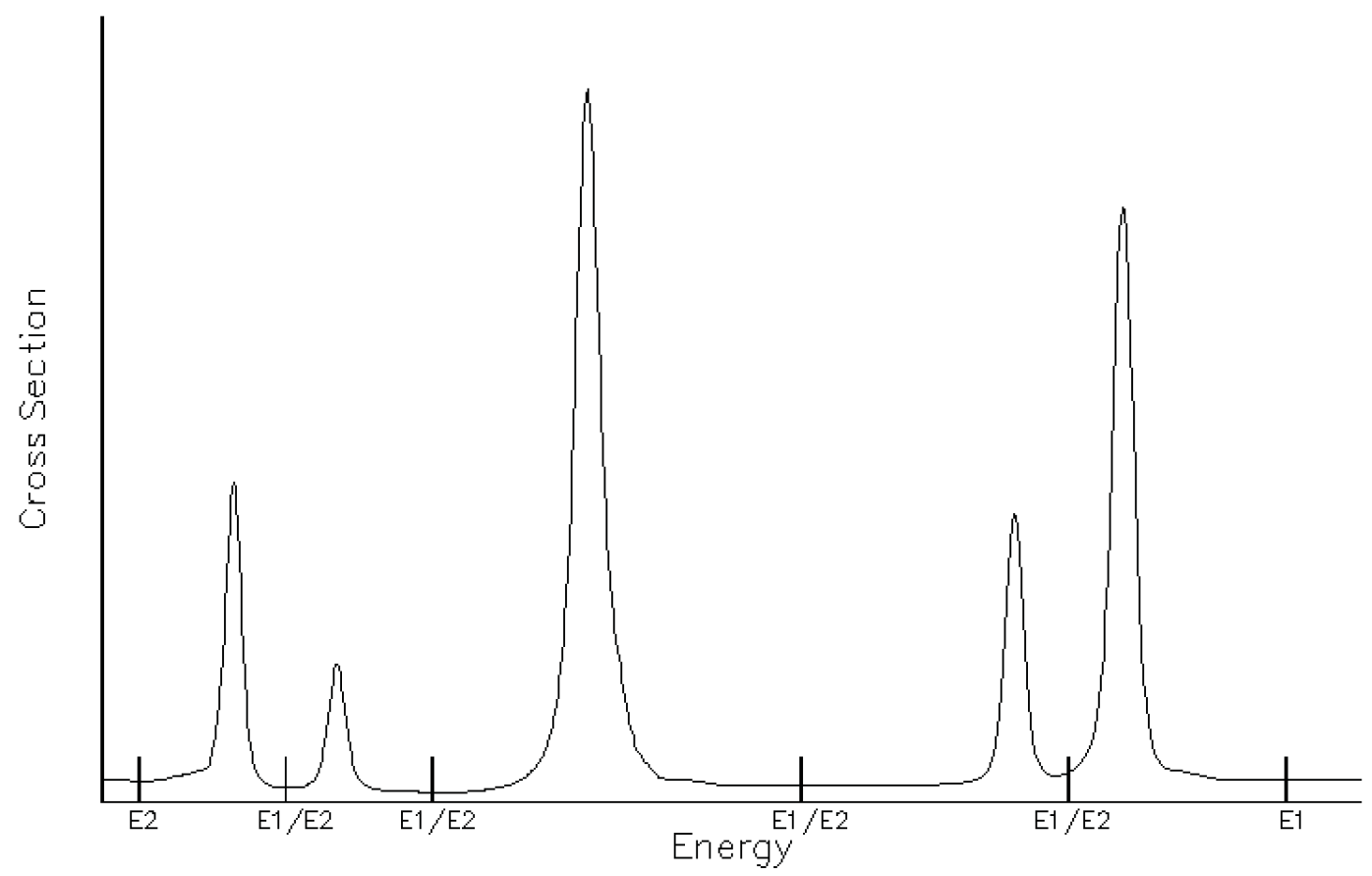

Figure 2. An example of Doppler broadened resonance cross sections.

\subsubsection{Determination of $E_{1} / E_{2}$ and the Number of Meshes for a Resonance}

The first energy, $E_{1}$, corresponds to the first fine group energy boundary encountered just below the upper limit of the highest resolved resonance energy range. It can be the user input at any fine group energy boundary lower than that. The next $\mathrm{E}_{1}\left(\right.$ or $\left.\mathrm{E}_{2}\right)$ is at the midpoint between the next two neighboring resonances, and so on. The lowest $\mathrm{E}_{2}$ is also adjusted to correspond to the fine group energy boundary. For each resonance, a resonance integration interval is formed by $E_{1}$ and $E_{2}$. Considering an equal lethargy interval solution, a mesh size and the number of mesh intervals within the resonance integration are calculated according to the following recipe, which was adopted from GAM-II code ${ }^{22}$ and modified.

$\mathrm{r}=5.0+0.5 \sqrt{K * 273.0 * E_{0} / A W R I} / \Gamma$

$\varepsilon=1.0 /\left[A W R * \operatorname{int}\left\{0.5 * r * \sqrt{1.0 /(K * 273.0 * A W R I) *\left|E_{0}\right|}+1.0\right\} *\right.$ fmult $]$

$\operatorname{mesh}=\frac{\log \left(E_{1} / E_{2}\right)}{\varepsilon}$

where: mesh is the number of mesh intervals, $\mathrm{K}$ is the Boltzmann constant $=8.6173 \mathrm{e}-5 \mathrm{eV} / \mathrm{K}$, the constant 273.0 defines the mesh reference temperature $\mathrm{T}_{0}$, AWRI is the ratio of mass of a particular isotope to that of a neutron, $\Gamma$ is the total resonance width, $E_{1}$ and $E_{2}$ are the high and low energy boundaries of the integration for a resonance, $\mathrm{E}_{0}$ is the resonance energy, and fmult is the user input control parameter to make mesh coarse or finer (default=1.0).

The number of mesh intervals is then made to be an even number so that the total number of mesh points become odd for Simpson's integration. The last mesh point of the previous resonance integration interval corresponds to the first mesh point of the present resonance integration interval, and so on. The lethargy mesh is generated independently for each resonance from midpoint to midpoint between resonances; therefore, the mesh spacing from resonance to resonance varies. The highest E1 and lowest E2 can be controlled by user input, other than the default values set in "reres.lib" and adjusted to the group boundaries in the code. The maximum allowable number of mesh intervals in a resonance 
integration interval is coded to be 1400 . When the admixed resonance absorbers are present, the Doppler broadened cross sections are generated over these mesh points.

\subsubsection{Absorber Narrow Resonance- Moderator Asymptotic Flux Approximation}

In the Narrow Resonance (NR) approximation, the resonances are so narrow that all moderators, including the absorber, can scatter past the resonance. The scattering cross section over most of this range is essentially just that for potential scattering $\sigma_{p}$. The integral term in Equation (56) becomes

$\frac{1}{1-\alpha_{k}} \int_{u-\varepsilon_{k}}^{u} F\left(u^{\prime}\right) \frac{\sigma_{s}^{k}\left(u^{\prime}\right)}{\sigma_{t}\left(u^{\prime}\right)} d u^{\prime}=\frac{\sigma_{p}^{k}}{1-\alpha_{k}} \cdot \int_{u-\varepsilon_{k}}^{u} \phi\left(u^{\prime}\right) d u^{\prime}=\frac{\sigma_{p}^{k}}{1-\alpha} \cdot \int_{u-\varepsilon_{k}}^{u} \frac{e^{u^{\prime}}}{E_{1}} d u^{\prime}=\sigma_{p}^{k} \frac{e^{u}}{E_{1}}$,

when the asymptotic flux, $\phi_{\text {asym }}[u(E)]=e^{u} / E_{1}=w(u)$, is applied.

The criteria to determine this approximation is when $\ln \left(\frac{E_{i}}{\alpha E_{i}}\right) / \varepsilon \leq 1001$.

In the thermal energy range, $\mathrm{E} \leq 5 \mathrm{kT}$, the flux shape is rather Maxwellian than $e^{u} / E_{l}{ }^{26}$

$\phi_{\text {asym }}[u(E)]=5.936526 /(k T)^{2} \cdot E_{1} e^{-u} e^{-\frac{E_{1} e^{-u}}{k T}}=w(u)$.

\subsubsection{Self-Shielding Elastic Scattering Transfer Matrix}

The solution of Equation (20) or (38) and (39) requires the self-shielded resolved resonance contributions to the elastic scatter matrix for the Legendre moments through $\mathrm{L}=3$ or $\mathrm{L}=1$, respectively. Departing from the previous versions, a simple normalization scheme is employed for this, utilizing the already available transfer matrix from Bondarenko self-shielded transfer matrix, $\sigma_{s, g^{\prime} \rightarrow g}^{l}=\left(\sigma_{s, g^{\prime} \rightarrow g}^{l}\right)_{\text {Bondarenko }} \cdot \frac{\left(\sigma_{s, g^{\prime}}^{0}\right)_{\text {Nordheim }}}{\left(\sigma_{s, g^{\prime}}^{0}\right)_{\text {Bondarenko }}}$.

\subsubsection{Numerical Integration with Simpson's Rule}

Combining the integral form, absorber narrow resonance-moderator asymptotic flux approximation, and the thermal upscattering term, the numerical integration is expressed as

$$
\begin{aligned}
& F(u)=\left\{1-(1-P(u)) \frac{\varepsilon}{3 \sigma_{t}(u)}\left[\left(\delta\left(N_{0}\right) \frac{\sigma_{s 0}(u)}{\alpha_{0}}+\sum_{i}^{3} \delta\left(N_{a_{i}}\right) \frac{\sigma_{s a_{i}}(u)}{\alpha_{a_{i}}}+\sum_{j}^{3} \delta\left(N_{m_{j}}\right) \frac{\sigma_{s m_{i}}}{\alpha_{m_{j}}}\right)\right]\right\}^{-1} . \\
& \left\{\begin{array}{l}
(1-P(u)) \varepsilon\left[\begin{array}{l}
\frac{\delta\left(N_{0}\right)}{3 \alpha_{0}} \stackrel{u}{u-n \varepsilon}^{u-\varepsilon} F\left(u^{\prime}\right) \frac{\sigma_{s o}\left(u^{\prime}\right)}{\sigma_{t}\left(u^{\prime}\right)}+\sum_{i}^{3} \frac{\delta\left(N_{a_{i}}\right)}{3 \alpha_{a_{i}}}{ }_{u-n \varepsilon}^{u-\varepsilon} F\left(u^{\prime}\right) \frac{\sigma_{s a_{i}}\left(u^{\prime}\right)}{\sigma_{t}\left(u^{\prime}\right)}+\sum_{j}^{3} \frac{\delta\left(N_{m_{j}}\right)}{3 \alpha_{a_{i}}} \prod_{u-n \varepsilon}^{u-\varepsilon} F\left(u^{\prime}\right) \frac{\sigma_{s m_{j}}\left(u^{\prime}\right)}{\sigma_{t}\left(u^{\prime}\right)} \\
+\delta(u p) \sum_{g^{\prime}} F_{g^{\prime}} \frac{\sigma_{s s^{\prime} \rightarrow g}^{u p s t}}{\sigma_{t g}} \frac{\Delta g^{\prime}}{\Delta g}
\end{array}\right] \\
+\left[(1-P(u))\left(\delta(N R) \sigma_{p_{0}}+\sum_{i}^{3} \delta\left(A_{a_{i}}\right) \sigma_{p a_{i}}+\sum_{j}^{3} \delta\left(A_{m_{j}}\right) \sigma_{p m_{j}}+P(u) \sigma_{t}(u)\right] w(u)\right.
\end{array}\right\}
\end{aligned}
$$

where $\delta\left(\mathrm{N}_{\mathrm{X}}\right)$ means Nordheim integral treatment application for the resonance absorber, admixed resonance absorbers, admixed moderators, $\delta(\mathrm{NR}), \delta\left(\mathrm{A}_{\mathrm{a}}\right), \delta\left(\mathrm{A}_{\mathrm{m}}\right)$ means application of absorber 
narrow resonance-moderator asymptotic flux approximation instead of Nordheim integral treatment, $\delta$ (up) means application of upscattering when thermal upscattering is present

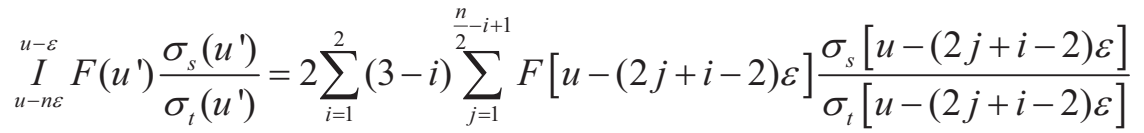

$$
\begin{aligned}
& +F(u-\varepsilon n) \frac{\sigma_{s}(u-\varepsilon n)}{\sigma_{t}(u-\varepsilon n)}
\end{aligned}
$$

is the Simpson's rule integration.

When thermal upscattering is present, iteration is needed. Since the convergence is rapid when the available flux in the code is utilized, only once more iteration is performed.

\subsubsection{Group Averaging}

Two options are available for converting the resonance integrals in Equation (53) into average multigroup cross sections, depending on whether one desires averages over the whole cell or just over the absorber lump. The average cross section is defined by

$$
\sigma_{x g}=\frac{E_{1} \int_{u_{i-1}}^{u i} F(u) \frac{\sigma_{x}(u)}{\sigma_{t}(u)} e^{-u} d u}{E_{1} \int_{u_{i-1}}^{u i} \phi(u) e^{-u} d u}
$$

where $\sigma_{\mathrm{x}}$ is the reaction cross section being averaged, $\mathrm{F}(\mathrm{u})$ is given by Equation (58), and $\sigma_{\mathrm{t}}$ is given by Equation (63). The reaction cross section is averaged over the absorber lump flux,

$\phi(\mathrm{u})=\mathrm{F}(\mathrm{u}) / \sigma_{\mathrm{t}}(\mathrm{u})=$ lump flux

After obtaining capture/fission and scattering cross sections from Nordheim treatment, these cross sections overwrite the previous cross sections.

\subsubsection{Dancoff-Ginsburg Correction Factor Calculation}

Computation of the Dancoff factor $\mathrm{C}$ for arbitrary shapes and spacing of lumps is very difficult. Most accurate determinations have been performed for regular arrays of cylindrical fuel pins. An external code like SUPERDAN can provide Dancoff factors. ${ }^{27}$ One option in COMBINE is an exact calculation described below. In the event that a precise calculation is not possible due to geometric irregularity or nonavailability, Bell offers an approximate formula based on the most general assumptions, which can be used as a last resort. Bell's formula can be written $\mathrm{as}^{28}$

$$
C \approx \frac{\lambda_{1}}{\frac{4 V_{1}}{S_{a}}+\lambda_{1}}
$$

where

$$
\begin{aligned}
& \mathrm{S}_{\mathrm{a}}=\text { surface area of the absorbing lump } \\
& \mathrm{V}_{1}=\text { the average external moderator volume per absorber lump } \\
& \lambda_{1}=\text { the external moderator mean-free-path in the same units as } \mathrm{V}_{1} \text { and } \mathrm{S}_{\mathrm{a}} .
\end{aligned}
$$

Equation (74) is deceptive in its simplicity considering the exact definition in Equation (75). It is exact in the limits of very large or very small lumps. The Rational Approximation for $\mathrm{P}_{\mathrm{o}}$ has been used in deriving Equation (74) along with other assumptions, so it should be used only as a first order 
approximation when no other method is available. Equation (74) can be used with any regular or irregular geometry and is independent of geometric details other than average values of $\mathrm{V}_{1}$ and $\mathrm{S}_{\mathrm{a}}$. A precise calculation of $\mathrm{C}$ for cylindrical pins is included in COMBINE and is described below.

The cylindrical pin Dancoff correction is computed in COMBINE following the methods of Gelling and Sauer. ${ }^{29}$ This correction may be calculated for an infinite lattice composed of cylindrical pins in either a rectangle or a hexagon. Pins are assumed to be black to resonance-energy neutrons.

The Dancoff correction is defined as: ${ }^{24}$

$C=\frac{\int d S \int \vec{n} \cdot \vec{\Omega} \exp \left[-\sum l(\vec{s}, \vec{\Omega})\right] d \Omega}{\int d S \int \vec{n} \cdot \vec{\Omega} d \Omega}$

where $l(\vec{s}, \vec{\Omega})$ is a chord that extends from the lump surface element dS in the direction $\vec{\Omega}$ through the moderator to the point of intersection with a neighboring lump. $\Sigma$ is the cross section for removal of neutrons from the energy range of the resonance and is the normal to the lump surface element.

In the case of a lattice composed of cylindrical pins, the Dancoff correction may be expressed as $C=\frac{4}{\pi} \int_{0}^{2 \pi} \frac{d \rho}{2} \int_{-1}^{1} \frac{d \xi}{2} K_{i 3}\left[\sum R_{0} \cdot S\right]$.

The parameters are defined in Figure 3, and

$K_{i 3}=\int_{0}^{\infty} \exp [-x \cosh (u)] \cosh ^{-3} u d u$

is the Bickley function of the third order. 


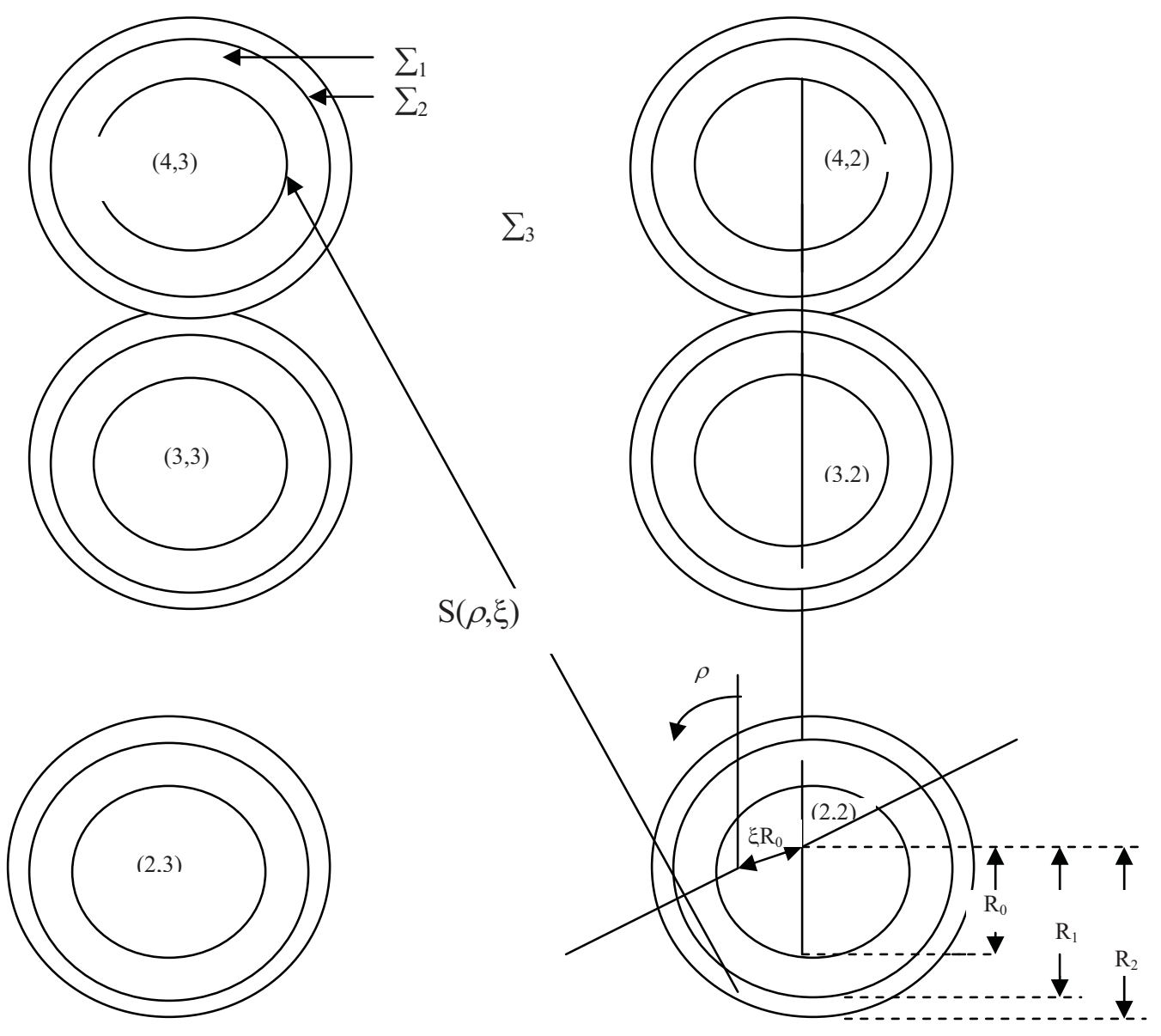

Figure 3. Parameters of the rectangular cell.

The two-dimensional lattice is represented exactly for a pin surrounded by up to four annular moderating regions and an external moderator. A library of pin configurations is contained within the code. A pin location is specified by the row $\mathrm{n}$ and the column $\mathrm{m}$, where $\mathrm{m}$ and $\mathrm{n}$ are in units of pitch.

Heterogeneity of the moderating region is accounted for by defining the argument of the Bickley function $^{29}$ as

$\sum R_{0} \cdot S=R_{0} \sum_{i=1}^{N} \sum_{i} S_{i}$

where $\mathrm{s}_{\mathrm{i}}$ is the length of the chord through region $\mathrm{i}$, having total cross section $\Sigma_{\mathrm{i}}$.

The integral in Equation (76) is evaluated using the trapezoidal approximation. Because of symmetry, the upper limit is taken as $\pi / 4$ for a rectangular lattice and $\pi / 6$ for a hexagonal lattice. The integral over $\rho$ is evaluated by using 20 equal angular intervals, and for each value of $\rho$ the integral over $\xi$ is evaluated by using 100 increments of equal length. For each set of $(\rho, \xi)$ values, the length of the chord $\mathrm{S}_{\mathrm{i}}$ through each region is calculated and the Bickely function is evaluated by the approximation

$K_{i 3}(x)=\frac{e^{-x}}{(0.6366198 x+1.6211389)^{1 / 2}} \cdot \frac{x^{2}+6.399407 x+5.066719}{x^{2}+6.766116 x+5.066719}$.

This approximation yields values of $k_{i 3}(x)$ to an accuracy of $\pm 0.05 \%$. 


\subsection{Computation of Thermal Disadvantage Factors}

The absorber lump appears black to neutrons at the peak resonance cross sections. For neutrons at thermal energies, the same lump is more than grey. As a consequence, corrections for thermal scattering are much more important. Amouyal and Benoist ${ }^{30}$ have developed integral transport theory for the calculation of thermal disadvantage factors in grey absorber lumps. Strawbridge ${ }^{31}$ has modified this theory to include a third clad region. The three region cell equations for the point wise disadvantage factors $\mathrm{W}_{\mathrm{i}}$ in a cylindrical cell are, ${ }^{30}$

$$
\frac{W\left(R_{0}\right)}{W_{0}}=1+\frac{\sum_{a 0}(E)}{\sum_{t 0}(E)}\left[\frac{1-P_{e}}{P_{e}}-R_{0} \sum_{t 0}(E)\right] \times \quad\left\{1+\alpha \frac{\sum_{s 0}(E)}{\sum_{t 0}(E)}+\beta\left[\frac{\sum_{s 0}(E)}{\sum_{t 0}(E)}\right]\right\}
$$

where

$\mathrm{W}_{\mathrm{o}} \quad=$ average energy dependent disadvantage factor in the cylindrical absorber rod

$\mathrm{W}\left(\mathrm{R}_{\mathrm{o}}\right)=$ energy dependent disadvantage factor at the surface of the absorber rod between the rod and the clad

$\sum_{\mathrm{a} 0}(\mathrm{E})=$ absorption cross section in the absorber rod

$\sum_{\mathrm{t} 0}(\mathrm{E})=$ total cross section in the absorber rod

$\sum_{\mathrm{s} 0}(\mathrm{E})=$ scattering cross section in the absorber rod

$\mathrm{R}_{0} \quad=$ radius of the absorber rod

$\mathrm{P}_{\mathrm{e}}=\mathrm{P}_{\mathrm{e}}\left[\bar{l}_{0}, \sum_{t 0}^{*}(E)\right]=$ the first flight escape probability in a cylindrical absorber rod at energy $\mathrm{E}$

where

$$
\bar{l}_{0}=2 \mathrm{R}_{\mathrm{o}}
$$

$\alpha, \beta=$ tabulated empirical factors to correct the first flight escape probability $\mathrm{P}_{\mathrm{e}}$ for the effects of scattering within the absorber rod.

Equation (80) resulted from considerations by A/B (Amouyal/Benoist) to adjust the first flight escape probability $\mathrm{P}_{\mathrm{e}}$ (correct for a black pin) for the effects of scattering in a grey pin. The basis $\mathrm{A} / \mathrm{B}$ thermal equation suppressing energy dependence, is

$$
\frac{W\left(R_{0}\right)}{W_{0}}=\frac{1}{P_{s}\left(\bar{l}_{0}, \sum_{t 0}\right)}-R_{0} \sum_{a 0}
$$

where $\mathrm{P}_{\mathrm{s}}$ is the escape probability for a single grey pin corrected for multiple scattering within the pin.

$$
P_{s}\left(\bar{l}_{0}, \sum_{t 0}\right)=\left(1+\frac{\sum_{a 0}}{\sum_{t 0}}\left\{A\left[1+\alpha \frac{\sum_{s 0}}{\sum_{t 0}}+\beta\left(\frac{\sum_{s 0}}{\sum_{t 0}}\right)^{2}\right]+R_{0 \Sigma_{t 0}}\right\}\right)^{-1}
$$

where

$$
\begin{aligned}
& A=\frac{1-P_{e}}{P_{e}}-R_{0} \sum_{t 0} \\
& P_{e}\left[\bar{l}_{o}, \sum_{t}(E)\right] \text { is obtained from the tabular lookup used for resolved resonances. }
\end{aligned}
$$


When Equation (82) is substituted into Equation (81) we obtain Equation (80). For a black pin, $\sum_{\mathrm{s}}=0$ and Equation (81) can be reduced to Wigner's Rational Approximation using the flat flux assumption.

$$
\frac{W\left(R_{0}\right)}{W_{0}}=\frac{1}{P_{0}\left(\bar{l}_{0}, \Sigma_{a 0}\right)}-R_{0} \sum_{a 0}=\frac{W_{1}}{W_{0}}-R_{0} \sum_{a 0}
$$

where $\frac{1}{P_{0}} \approx \frac{W_{1}}{W_{0}}$ and $\mathrm{W}_{1}$ is the asymptotic average flux in the moderator region outside the black absorber rod. Since the flat flux assumption implies a discontinuity in the flux at the boundary

$$
\begin{aligned}
& W\left(R_{0}\right)=\frac{W_{1}+W_{0}}{2} \\
& \frac{W_{1}+W_{0}}{2 W_{0}}+R_{0} \Sigma_{a 0}=\frac{1}{P_{0}} \\
& \frac{W_{1}}{W_{0}}+1+2 R_{0} \Sigma_{a 0}=\frac{2}{P_{0}} \\
& P_{0}=\frac{1}{1+\bar{l}_{0} \Sigma_{a 0}} \equiv \text { Rational Approximation, }
\end{aligned}
$$

which is the Wigner's Rational Approximation equation when $\sum_{\mathrm{a} 0}$ replaces $\sum_{\mathrm{t} 0}$. The $\mathrm{ABH}^{30}$ equation for the external moderator Region 1, with absorption in the moderator included and an additional clad Region 2 separating the absorber and moderator, is

$$
\frac{W_{1}}{W_{0}}=\frac{W\left(R_{0}\right)}{W_{0}}+\frac{\Delta W_{2}}{W_{0}}+\frac{3}{2} R_{0}^{2} \sum_{a 0}\left[1+\frac{V_{2}}{V_{0}} \frac{\sum_{a 2}}{\sum_{a 0}} \frac{W_{2}}{W_{0}}\right] F
$$

where

$$
\begin{aligned}
& F=G \sum_{t 1}+\frac{1}{R_{2}}\left(\lambda_{1}-\frac{2}{3}\right) \\
& G=\frac{R_{1}^{2}}{R_{1}^{2}-R_{2}^{2}}\left[\frac{R_{1}^{2}}{R_{1}^{2}-R_{2}^{2}} \ln \left(\frac{R_{1}}{R_{2}}\right)-\frac{3}{4}+\frac{R_{2}^{2}}{4 R_{1}^{2}}\right] \\
& \lambda_{1}=0.710446\left[\frac{1+1.5207587 \frac{3 D_{1}}{2 R_{1}}}{1+0.8103127 \frac{3 D_{1}}{2 R_{1}}}\right] \text { (the extrapolation length for the inner surface of the moderator } \\
& \text { region })^{32} \\
& \mathrm{~W}_{1}=\text { disadvantage weight for the external moderator region } \\
& \mathrm{W}_{2}=\text { disadvantage weight for the clad region } \\
& \sum_{\mathrm{t} 1}=\text { the total cross section in the moderator region } \\
& \sum_{\mathrm{a} 2}=\text { the absorption cross section in the clad region } \\
& \mathrm{R}_{2}=\text { the outer radius of the clad region } \\
& \mathrm{R}_{1}=\text { the outer radius of the moderator region }
\end{aligned}
$$


$\mathrm{D}_{1}=$ the diffusion coefficient in the moderator region.

The equation for the clad disadvantage factor $\mathrm{W}_{2}$ is

$\frac{W_{2}}{W_{0}}=\frac{W\left(R_{0}\right)}{W_{0}}+\frac{\Delta W_{2}}{2 W_{0}}$

where

$\frac{\Delta W_{2}}{2}=$ average incremental rise in the flux across the clad region.

This can be obtained from simple diffusion theory by balancing the volume absorption rate in the absorbing rod to the diffusion current entering the rod,

$W_{0} \sum_{a 0} V_{0}=\left.D_{2} \frac{\Delta W_{2}}{\Delta R}\right|_{R_{0}} 2 \pi R_{0}$

$\frac{\Delta W_{2}}{W_{0}}=\frac{\sum_{a 0} \pi R_{0}^{2}\left(R_{2}-R_{0}\right)}{2 \pi R_{0} D_{2}}$

$\frac{\Delta W_{2}}{2 W_{0}}=\frac{\sum_{a 0} R_{0}\left(R_{2}-R_{0}\right)}{4 D_{2}}$

where $\mathrm{D}_{2}$ is the diffusion coefficient in the clad region.

The weights $\mathrm{W}_{0}, \mathrm{~W}_{1}$, and $\mathrm{W}_{2}$ are normalized at each energy group as

$W_{0} V F_{0}+W_{2} V F_{2}+W_{1} V F_{1}=1$

where $\mathrm{VF}_{0}, \mathrm{VF}_{2}$, and $\mathrm{VF}_{1}$ are the volume fractions of the absorber, clad and moderator regions, respectively.

Dividing Equation (86) by $\mathrm{W}_{0}$, we have

$\frac{1}{W_{0}}=V F_{0}+\left[\frac{W_{2}}{W_{0}}\right] V F_{2}+\left[\frac{W_{1}}{W_{0}}\right] V F_{1}$.

When Equations (80), (84), and (85) are solved for $\mathrm{W}_{2} / \mathrm{W}_{0}$, and Equation (83) is solved for $\mathrm{W}_{1} / \mathrm{W}_{0}$, Equation (87) is solved for $\mathrm{W}_{0}$. Then

$$
\begin{aligned}
& W_{2}=\left(\frac{W_{2}}{W_{0}}\right) W_{0} \\
& W_{1}=\left(\frac{W_{1}}{W_{0}}\right) W_{0} .
\end{aligned}
$$

\subsubsection{Slab Geometry Disadvantage Weights}

The equations for plate type geometry are taken from Theys ${ }^{33}$ and are similar, but differ due to the difference in divergence.

$$
\frac{W\left(X_{0}\right)}{W_{0}}=1+\frac{\sum_{a 0}}{\sum_{t 0}} A\left[1+\alpha \frac{\sum_{s 0}}{\sum_{t 0}}+\beta\left[\frac{\sum_{s 0}}{\sum_{t 0}}\right]^{2}\right]
$$


where

$$
\begin{aligned}
& \mathrm{A}=\frac{1-P_{e}}{P_{e}}-2 X_{0} \sum_{t 0} \\
& \frac{W_{1}}{W_{0}}=\frac{W\left(X_{0}\right)}{W_{0}}+\frac{\Delta W_{2}}{W_{0}}+\sum_{a 0} X_{0}\left[\sum_{1}^{*}\left(X_{1}-X_{2}\right)+3 \lambda_{1}-2\right] \\
& \frac{\Delta W_{2}}{W_{0}}=\frac{\sum_{a 0} X_{0}\left(X_{2}-X_{0}\right)}{2 D_{2}} \\
& \frac{W_{2}}{W_{0}}=\frac{W\left(X_{0}\right)}{W_{0}}+\frac{\Delta W_{2}}{2 W_{0}}
\end{aligned}
$$

where $\lambda_{1}=0.710446$ is the extrapolation length for the inner surface of the moderator region and $\Sigma_{1}^{*}$ is the moderator region transport cross section. $\mathrm{X}_{0}, \mathrm{X}_{1}$, and $\mathrm{X}_{2}$ plate geometry dimensions and all other quantities are defined as in the corresponding cylindrical geometry equations. Equations (86) to (89) are then solved for the disadvantage weights.

\subsubsection{Self-Shielding Factors}

Disadvantage factor weighted self-shielding factors SSF (ISO,g) are calculated at each energy group $\mathrm{g}$ for every isotope ISO in the 3 region cell.

$\mathrm{SSF}(\mathrm{ISO}, \mathrm{g})=\frac{\sum_{j=1}^{3} W_{j}^{g} V F_{j} N_{j}^{I S O}}{D E N S(I S O)}$

where

$\operatorname{DENS}(\mathrm{ISO})=\sum_{j=1}^{3} V F_{j} N_{j}^{I S O}$

$V F_{j} \quad=$ volume fraction of the $\mathrm{j}$ region in the cell

$N_{j}^{I S O}=$ the isotopic density in each region of the cell.

The self-shielding factors SSF(ISO,g) are printed to the output. Effective cell-average microscopic cross sections of reaction type $\mathrm{x}$ at each mesh point are obtained from

$$
\left\langle\sigma_{x, g}^{I S O}\right\rangle=S S F(I S O, g) \sigma_{x, g}^{I S O}
$$

The fine group cross sections in the thermal energy range $(1.0 \mathrm{e}-5-5.0435 \mathrm{eV})$ are replaced by those self-shielded with the $\mathrm{ABH}$ method in the three region geometry when this option is requested.

\subsection{Age Equations}

An option to perform an age calculation utilizing a moments method, which was available in the GAM-1 code, has been retained in COMBINE. The derivation of this method is described in Reference 13 and has been omitted here. However, the final equations solved for the age are briefly described below. In multigroup form, the ith group moment equations for $\phi_{00_{i}}, \phi_{11_{i}}$, and $\phi_{20_{i}}$ are

$\sum_{t_{i}} \phi_{00_{i}}=\sum_{j=l}^{i}\left(\sum_{s 0 j \rightarrow i}+\sum_{i n j \rightarrow i}+2 \sum_{n, 2 n j \rightarrow i}\right) \phi_{00_{j}} \Delta_{j}+S_{i}$ 
$\sum_{t_{i}} \phi_{11_{i}}=\sum_{j=l}^{i} \sum_{s l j \rightarrow i}+\phi_{11_{j}} \Delta_{j}+\phi_{00_{i}}$

$\sum_{t_{i}} \phi_{20_{i}}=\sum_{j=l}^{i}\left(\sum_{s 0 j \rightarrow i}+\sum_{i n j \rightarrow i}+2 \sum_{n, 2 n j \rightarrow i}\right) \phi_{20_{j}} \Delta_{j}+\frac{\phi_{11 i}}{3}$

where all cross section definitions are the same as those described previously for the B-1 solution.

Solving each moment equation for the $i t h$ group we have the final solution equations:

$\phi_{00_{i}}=\frac{\sum_{t_{i}} \phi_{00_{i}}=\sum_{j=1}^{i}\left(\sum_{s 0 j \rightarrow i}+\sum_{i n j \rightarrow i}+2 \sum_{n, 2 n j \rightarrow i}\right) \phi_{00_{j}} \Delta_{j}+S_{i}}{\sum_{j=i+1}^{N O G}\left(\sum_{s 0_{i \rightarrow j}}+\sum_{i n_{i \rightarrow j}}+2 \sum_{n, 2 n_{i \rightarrow j}}\right) \Delta_{j}-\sum_{n, 2 n_{i \rightarrow i}} \Delta_{i}}$

$\phi_{11_{i}}=\frac{\phi_{00_{i}}+\sum_{j=1}^{i+1} \sum_{s l j \rightarrow i}+\sum_{i n_{j \rightarrow i}} \phi_{11_{j}} \Delta_{j}}{\sum_{t i}-\sum_{s I_{i \rightarrow i}} \Delta_{i}}$

$\phi_{20_{i}}=\frac{\frac{\phi_{11_{i}}}{3}+\sum_{j=1}^{i-1}\left(\sum_{s 0 j \rightarrow i}+\sum_{i n_{j} \rightarrow i}+2 \sum_{n, 2 n_{j} \rightarrow i}\right) \phi_{20_{j}} \Delta_{j}}{\sum_{j=i+1}^{N O G}\left(\sum_{s 0_{i} \rightarrow j}+\sum_{i n_{i \rightarrow j}}+2 \sum_{n, 2 n_{i \rightarrow j}}\right) \Delta_{j}-\sum_{n, 2 n_{i \rightarrow i}} \Delta_{i}}$

$\tau_{i}=\frac{\phi_{20_{i}}}{\phi_{00_{i}}}=$ age to group i.

\subsection{Transport Approximations}

The difference between the $S_{\mathrm{N}}$ and $P_{\mathrm{N}}$ require ${ }^{10}$

$\sigma_{l g^{\prime} \rightarrow g}^{S N}=\sigma_{l g^{\prime} \rightarrow g}^{P N}$ for $g^{\prime} \neq g$

and

$\sigma_{l g \rightarrow g}^{S N}=\sigma_{l g \rightarrow g}^{P N}-\sigma_{l t g}^{P N}+\sigma_{g}^{S N}$

where $\sigma_{g}^{S N}$ is not determined. The choice of $\sigma_{g}^{S N}$ gives rise to a "transport approximation" and various recipes are in use:

- Consistent-P approximation is

$$
\sigma_{\ell g \rightarrow g}^{S N}=\sigma_{\ell g \rightarrow g}^{P N}-\left(\sigma_{\ell t g}^{P N}-\sigma_{0 t g}^{P N}\right)
$$

- Inconsistent-P approximation is

$$
\sigma_{\ell g \rightarrow g}^{S N}=\sigma_{\ell g \rightarrow g}^{P N}-\left(\sigma_{\ell t g}^{P N}-\sigma_{N+1 t g}^{P N}\right)
$$


- Diagonal transport approximation

$$
\sigma_{\ell g \rightarrow g}^{S N}=\sigma_{\ell g \rightarrow g}^{P N}-\left(\sigma_{\ell t g}^{P N}-\sigma_{N+1 g}^{P N}+\sigma_{N+1 g \rightarrow g}^{P N}\right)
$$

- Bell-Hansen Sandmeier or extended transport approximation

$$
\sigma_{\ell g \rightarrow g}^{S N}=\sigma_{\ell g \rightarrow g}^{P N}-\left(\sigma_{\ell t g}^{P N}-\sigma_{N+1 t g}^{P N}+\sum_{g^{\prime}} \sigma_{N+1 g \rightarrow g^{\prime}}^{P N}\right)
$$

When the $P_{\mathrm{N}}$ to $S_{\mathrm{N}}$ correction is requested in the COMBINE7.1 input, the average of the above approximations is used for B-1 calculation but only the consistent-P approximation is used for B-3 calculation. 


\section{ONE-DIMENSIONAL TRANSPORT CALCULATION}

The ANISN/PC code for one-dimensional transport theory calculations is incorporated in the COMBINE7.1. The ANISN portion solves the multigroup finite difference discrete ordinates equations with anistropic scattering by using the fine group cross section constants, generated for the stacked zerodimensional or infinite media. Thus calculated fluxes are used to spatially collapse the fine group constants to better reflect the spatial self-shielding. In the discrete oridinates method, the neutron transport equation is solved in a discrete set of directions only. Angular integrals are then approximated by sums over discrete directions and angular derivatives by differences. The detail of ANISN/PC code can be found in the ANISN/PC manual ${ }^{14}$ pdf file, included as a part of COMBINE7.1 code package.

Currently, the ANISN inputs are externally provided in the COMBINE7.1 batch run stream as described in the input description. The spatial coalescing formulas are separately described in the user's guide section. 


\section{USER'S GUIDE}

\subsection{Derivation of Diffusion Coefficient and Transport Cross Section}

Although it is not evident from a cursory examination of B-1 equations, the mathematical structure of the system of equations resulting in Equations (30) and (31) is predetermined by the assumptions embodied in Equation (5). The assumptions, mathematically valid for a bare critical system satisfying simple diffusion boundary conditions, are characteristic of the broad generalities leading to the so-called First Fundamental Theorem of Reactor Theory. ${ }^{18}$ The usual approach to the B-N energy-dependent equations involves taking the Fourier transform of the steady-state transport equation for a slab, with the implication that diffusion boundary conditions are somehow applicable to any order $\mathrm{N}$.

The conceptual difficulty with this approach is that the resultant equations obscure any direct relationship between the spatial and energy dependence of the variables, unless the resultant equations are first subjected to Fourier inversion. Such a relationship is required to derive a rigorous definition of the energy-dependent diffusion coefficient by means of Fick's law. The procedure of deriving the B-1 equations by use of Equation (5) and diffusion theory boundary conditions for the scalar flux enables one to accomplish this end in a straightforward way.

Equation (5) establishes the desired relationship between the variables subject to the boundary conditions at the extrapolated boundary of the slab. Only the real part of $\mathrm{e}^{-\mathrm{iBx}}$ satisfies the boundary conditions for the spatially dependent scalar flux. The scalar flux is obtained from Equations (5), (8), and (9) in the conventional manner.

$$
\begin{aligned}
& \Phi(x, \mu, E)=e^{-i B x} \psi(B, \mu, E) \\
& \Phi_{0}(x, E)=\int_{-1}^{1} \Phi(x, \mu, E) d \mu=e^{-i B x} \int_{-1}^{1} \psi(B, \mu, E) d \mu=e^{-i B x} \phi_{0}(B, E)
\end{aligned}
$$

From Fick's law, we obtain the relationship between the scalar flux and current

$$
\vec{J}(x, E)=-D(E) \vec{\nabla} \Phi_{0}(x, E)=-\vec{k} D(E) \frac{d}{d x} \Phi_{0}(x, E)=D(E) B \phi_{0}(B, E) i \vec{k} e^{-i B x} .
$$

where $\vec{k}$ is the unit vector in the $\mathrm{x}$ direction.

Therefore, $\vec{J}(x, E)$ is also separable in space and energy, a result consistent with Equation (5).

$$
\therefore \vec{J}(x, E)=J(B, E) i \bar{k} e^{-i B x}
$$

where $J(B, E)=D(E) B \phi_{0}(B, E)$.

It has been pointed out that Equation (110) does not give a clear-cut relationship between J(E) and $\phi_{1}(\mathrm{E})$ in Equations (30) and (31) since Fick's law is perfectly general. An alternate method of obtaining Equation (110) will be given, which helps clear up any ambiguity that may exist. Using the same assumptions and utilizing Equations (5), (8), and (9), we have

$$
\begin{aligned}
& \vec{J}^{*}(x, E)=\vec{k} \int_{-1}^{1} \mu \Phi(x, \mu, E) d \mu=\vec{k} \int_{-1}^{1} \mu e^{-i B x} \psi(B, \mu, E) d \mu=i \vec{k} \phi_{1}(B, E) e^{-i B x} \text { when B is real, } \\
& =\bar{k} \phi_{1}(B, E) e^{-i B x} \text { when B is imaginary. }
\end{aligned}
$$

Introducing Fick's law without reference to the scalar flux boundary conditions we have 
$\vec{J}^{*}(x, E)=-D(E) \vec{\nabla} \Phi_{0}(x, E)=D(E) B \phi_{0}(B, E) i \vec{k} e^{-i B x}$

Now equate $\vec{J}^{*}(x, E)$ from the two preceding equations to obtain a result similar to Equation (110) as $\vec{J}^{*}(x, E)=\phi_{1}(B, E) i \vec{k} e^{-i B x}=D(E) B \phi_{0}(B, E) i \vec{k} e^{-i B x}$ when B is real, $=\phi_{1}(B, E) \vec{k} e^{-i B x}=D(E) B \phi_{0}(B, E) i \vec{k} e^{-i B x}$ when B is imaginary;

$\therefore \vec{J}(B, E)=\phi_{1}(B, E)=D(E) B \phi_{0}(B, E)$ when B is real,

$=i D(E) B \phi_{0}(B, E)$ when $\mathrm{B}$ is imaginary.

From Equation (107), $D(E)$ is defined as

$D(E)=\frac{\phi_{1}(B, E)}{B \phi_{0}(B, E)}$ when B is real,

$=\frac{\phi_{1}(B, E)}{i B \phi_{0}(B, E)}$ when $\mathrm{B}$ is imaginary.

Equation (112) gives an unambiguous definition of the diffusion coefficient within the framework of the B-1 equations for a bare slab. The definition of the B-1 transport cross section at energy $\mathrm{E}$ in the domain of Fick's law is

$$
\begin{aligned}
& \sum_{t r}(E)=\frac{1}{3 D(E)}=\frac{B \phi_{0}(B, E)}{3 \phi_{1}(B, E)} \text { when B is real, } \\
& \sum_{t r}(E)=\frac{1}{3 D(E)}=\frac{i B \phi_{0}(B, E)}{3 \phi_{1}(B, E)} \text { when B is imaginary. }
\end{aligned}
$$

In order to use Equation (113), the current from the solution run needs to be nonzero. Thus, when a zero buckling is input to the code, neither the diffusion coefficient nor the transport cross section is calculated. This situation corresponds to an infinite medium problem for which there is no net neutron current flow. If a diffusion coefficient is desired for a near infinite medium situation, a small but finite buckling input to the code will produce the desired results.

\subsection{Summary Edits for Broad Group Constants}

The equations for calculating the average cross sections are presented in this section in as complete a manners practical. This should facilitate the proper use of the average cross sections generated by the spectrum code. ${ }^{3,4}$

The COMBINE spectrum, cross-section library tape contains data tabulated for 167 fine lethargy groups. In a normal spectrum problem, the data are coalesced into a broad group structure, specified by the user, containing one or more fine groups per broad group. The coalescing is performed by utilizing 167 fine group fluxes and/or currents as the weighting functions. These energy dependent fluxes and currents may either be calculated for a particular reactor system or taken from an immediately preceding problem. The latter type of calculation is referred to as a cell calculation. The output from a spectrum problem normally includes average macroscopic cross-section data and may include average microscopic data for each material in the macroscopic calculation, if desired. As an alternative to the normal output, the user may request the calculation of macroscopic effective diffusion theory constants (blackness theory) in lieu of the normal cross section output. 
Figure 4 illustrates the notation used in describing the average cross-section data and the relation of the fine-group data to the broad-group data generated in a spectrum problem. In Figure 4, broad group $G$ contains the fine groups I through $\mathrm{I}+\mathrm{N}$ and all averages for broad group $\mathrm{G}$ are performed over this range of fine groups.

In the notation used above, NOG refers to the number of fine groups and NOAG refers to the number of broad groups.

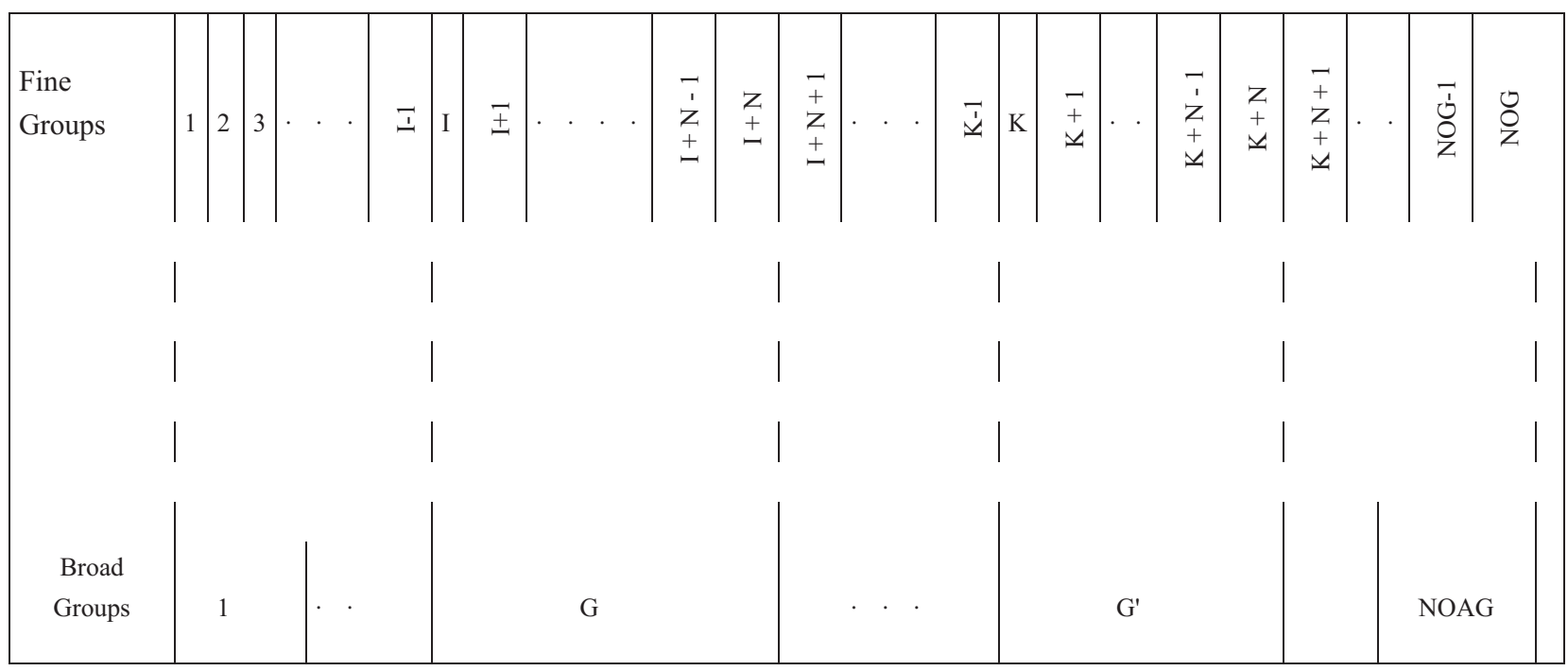

Figure 4. Spectrum group structure.

The average cross sections are determined by the familiar relation

$\bar{\Sigma}=\frac{\int_{E} \sum(E) w(E) d E}{\int_{E} w(E) d E}$

where $\bar{\Sigma}$ represents the energy dependent cross section $\Sigma(\mathrm{E})$, and $\mathrm{w}(\mathrm{E})$ is the energy dependent weighting function, normally either the fluxes or the currents. In the actual calculation, the continuous functions of the cross section and weighting parameter are represented by 167 fine group average values associated with a lethargy width, $\Delta_{\mathrm{i}}$. Thus,

$$
\bar{\Sigma}=\frac{\sum_{i} \sum_{i} w_{i} \Delta_{i}}{\sum_{i} w_{i} \Delta_{i}} .
$$

In all cases, the integrations are performed utilizing the trapezoidal rule.

\subsubsection{Average Macroscopic Cross Sections}

The macroscopic cross-section data generated by the COMBINE7.1 code is described below in considerable detail to facilitate its proper use. In all cases, the average quantity has been defined for the broad group $\mathrm{G}$ where $\sum_{i}\left(\mathrm{~cm}^{-1}\right)=\sum_{k=1}^{N O I} N_{k} \sigma_{i}^{k}=>$ macroscopic cross section for $\mathrm{i}^{\text {th }}$ fine group of a system containing NOI materials 
where $N_{k}$ and $\sigma_{i}^{k}$ are the atom density (\# of atoms $\times 10^{-24}$ per cubic centimeter) and microscopic cross section (barns/atom), respectively, for the $k^{\text {th }}$ material and

$\Delta_{\mathrm{i}} \quad=>$ lethargy width of fine group $\mathrm{i}$

$\phi_{\mathrm{i}} \quad=>$ average scalar flux in fine group $\mathrm{i}$

$\mathrm{J}_{\mathrm{i}} \quad=>$ average current $\left(\mathrm{P}_{\mathrm{i}}\right.$ flux $)$ in fine group $\mathrm{i}$

$\psi_{\mathrm{i}} \quad=>$ average $\mathrm{P}_{2}$ flux for group $\mathrm{i}$

$\chi_{\mathrm{i}}=>$ average $\mathrm{P}_{3}$ flux for group $\mathrm{i}$

$\bar{\Sigma}_{x_{G}}=>$ average cross section for broad group $\mathrm{G}$ for a particular reaction denoted by $x$

$\phi_{\mathrm{G}} \Rightarrow>$ integrated scalar flux for group $\mathrm{G}$

$\mathrm{J}_{\mathrm{G}} \Rightarrow>$ integrated current $\left(\mathrm{P}_{1}\right.$ flux $)$ for group $\mathrm{G}$

$\psi_{\mathrm{G}} \Rightarrow$ integrated $\mathrm{P}_{2}$ flux for group $\mathrm{G}$

$\chi_{\mathrm{G}}=>$ integrated $\mathrm{P}_{3}$ flux for group $\mathrm{G}$.

The integrated fluxes for broad group $\mathrm{G}$ are calculated as

$\Phi_{G}=\sum_{i=I}^{i+N} \phi_{i} \Delta_{i}$

$J_{G}=\sum_{i=I}^{i+N} J_{i} \Delta_{i}$

$\psi_{G}=\sum_{i=I}^{i+N} \psi_{i} \Delta_{i}$

$\chi_{G}=\sum_{i=I}^{i+N} \chi_{i} \Delta_{i}$

Average absorption cross section

$\bar{\Sigma}_{a_{G}}=\frac{\sum_{i=I}^{I+N} \sum_{a_{i}} \phi_{i} \Delta_{i}}{\Phi_{G}}$

Average fission cross section

$\bar{\Sigma}_{f}=\frac{\sum_{i=I}^{I+N} \sum_{f_{i}} \phi_{i} \Delta_{i}}{\Phi_{G}}$

Average number of neutrons per fission times the fission cross section

$v \bar{\Sigma}_{f}=\frac{\sum_{i=I}^{I+N} v_{i} \sum_{f_{i}} \phi_{i} \Delta_{i}}{\Phi_{G}}$ 
Average number of neutrons produced per fission

$\bar{v}_{G}=\frac{\overline{v \sum}_{f_{G}}}{\bar{\Sigma}_{f_{G}}}$

Average capture cross section

$\bar{\Sigma}_{c_{G}}=\bar{\Sigma}_{a_{G}}-\bar{\Sigma}_{f_{G}}$

Average elastic, inelastic, $(n, 2 n),(n, 3 n)$, or $(n, 4 n)$ cross section for isotropic transfer from group $G$ to $G$

$\bar{\Sigma}_{x_{G \rightarrow G^{\prime}}}=\frac{\sum_{i=I}^{I+N} \sum_{k=K}^{K+N} \sum_{x_{i \rightarrow k}} \phi_{i} \Delta_{i}}{\Phi_{G}} \quad x_{i \rightarrow k}=\left\{\begin{array}{l}s_{0} \text { (elastic) } \\ i n \text { (inelastic) } \\ n, 2 n \text { reactions } \\ n, 3 n \text { reactions } \\ n, 4 n \text { reactions }\end{array}\right.$

Average linearly anisotropic component of elastic or inelastic transfer cross section from group $G$ to $G^{\prime}$

$\bar{\Sigma}_{x 1_{G \rightarrow G^{\prime}}}=\frac{\sum_{i=I}^{I+N} \sum_{k=K}^{K+N} \sum_{x_{i \rightarrow k}} J_{i} \Delta_{i}}{J_{G}} \quad x_{i \rightarrow k}=\left\{\begin{array}{l}s_{i} \text { (elastic) } \\ \text { in (inelastic) }\end{array}\right.$

Average $P_{2}$ component of the elastic transfer cross section from group $G$ to $G$

$\bar{\Sigma}_{s 2_{G \rightarrow G^{\prime}}}=\frac{\sum_{i=I}^{I+N} \sum_{k=K}^{K+N} \sum_{2_{i \rightarrow k}} \psi_{i} \Delta_{i}}{\psi_{G}}$

Average $P_{3}$ component of the elastic transfer cross section from group $G$ to $G$

$\bar{\sum}_{s 3_{G \rightarrow G^{\prime}}}=\frac{\sum_{i=I}^{I+N} \sum_{k=K}^{K+N} \sum_{3_{i \rightarrow k}} \chi_{i} \Delta_{i}}{\chi_{G}}$

Average total transfer cross section for transfer from group $G$ to $G$

$\bar{\sum}_{t t_{G \rightarrow G^{\prime}}}=\bar{\sum}_{s 0_{G \rightarrow G^{\prime}}}+\bar{\sum}_{i n_{G \rightarrow G^{\prime}}}+2 \bar{\sum}_{n, 2 n_{G \rightarrow G^{\prime}}}+3 \bar{\sum}_{n, 3 n_{G \rightarrow G^{\prime}}}+4 \bar{\sum}_{n, 4 n_{G \rightarrow G^{\prime}}}$

Average of isotropic elastic, inelastic, $(n, 2 n),(n, 3 n)$, or $(n, 4 n)$ total scattering cross section

$\bar{\Sigma}_{t_{x G}}=\sum_{G^{\prime}=G}^{N O A G} \bar{\Sigma}_{x_{G \rightarrow G^{\prime}}} \quad \chi_{G \rightarrow G^{\prime}}=\left\{\begin{array}{l}s_{0} \text { (elastic) } \\ \text { in } \text { (inelastic) } \\ n, 2 n \text { reactions } \\ n, 3 n \text { reactions } \\ n, 4 n \text { reactions }\end{array}\right.$

Average linearly anisotropic component of the elastic total scattering cross section

$\bar{\Sigma}_{t_{s 1_{G}}}=\sum_{G^{\prime}=G}^{N O A G} \bar{\Sigma}_{1_{G \rightarrow G^{\prime}}}$ 
Average out-scatter cross section

$\bar{\Sigma}_{o u t_{G}}=\sum_{G^{\prime}=G+1}^{N O A G}\left[\bar{\sum}_{s 0_{G \rightarrow G^{\prime}}}+\bar{\sum}_{i n_{G \rightarrow G^{\prime}}}+\bar{\sum}_{n, 2 n_{G \rightarrow G^{\prime}}}+\bar{\sum}_{n, 3 n_{G \rightarrow G^{\prime}}}+\bar{\sum}_{n, 4 n_{G \rightarrow G^{\prime}}}\right]+\sum_{G^{\prime}=G-\max u p}^{G-1} \bar{\sum}_{u p_{G \rightarrow G^{\prime}}}$

Average removal cross section

$\bar{\Sigma}_{r_{G}}=\bar{\Sigma}_{a_{G}}+\sum_{\text {out }}+\sum_{G^{\prime}=G-\max u p}^{G-1} \bar{\sum}_{u p_{G \rightarrow G^{\prime}}}$

Average total cross section

$\bar{\Sigma}_{t_{G}}=\bar{\Sigma}_{a_{G}}+\bar{\Sigma}_{s 0_{G}}+\bar{\sum}_{i n_{G}}+\bar{\Sigma}_{n, 2 n_{G}}+\bar{\Sigma}_{n, 3 n_{G}}+\bar{\Sigma}_{n, 4 n_{G}}$

Integrated fission source

$S_{G}=\sum_{i=I}^{I+N} S_{i}$

Average inverse velocity

$(\overline{1} / \mathrm{v})_{G}=\frac{\sum_{i=1}^{I+N}(1 / \mathrm{v})_{i} \Phi_{i} \Delta_{i}}{\Phi_{G}}$

Average diffusion coefficient (from Fick's law)

$\bar{D}_{G}=\frac{J_{G}}{B \Phi_{G}}$

Average transport cross section

$\bar{\Sigma}_{t r_{G}}=\frac{1}{3 \bar{D}_{G}}=\frac{B \Phi_{G}}{3 J_{G}}$

If a cell calculation is performed - a spectrum obtained from a previous problem is used as a weighting function to generate average cross sections for another material - the diffusion coefficients and transport cross sections are defined in terms of cross sections of the material being averaged. An appropriate definition can be obtained from Equation (37). By first solving Equation (37) for $\frac{B}{3} \phi$, and 
then dividing both sides by $\mathrm{J}_{\mathrm{i}}\left(\phi_{1}\right)$. We then have

$$
\bar{\Sigma}_{{ }_{t r_{\text {cell }}}}=\frac{\gamma^{g} \sum_{t}^{g} \Phi_{1}^{g}-\sum_{g^{\prime}=1}^{G} \sum_{s 1}^{g^{\prime} \rightarrow g} \Phi_{1}^{g} \frac{\Delta g^{\prime}}{\Delta g}}{\Phi_{1}^{g}}
$$

and

$$
\bar{D}_{G_{\text {cell }}}=\frac{1}{3 \bar{\Sigma}_{{ }_{t G_{\text {cell }}}}} \text {. }
$$

In addition to the usual average cross-section data described above, some other miscellaneous cross-section data are calculated and printed out for the user. These quantities include the absorption and out-scatter cross sections for each broad group corrected for the $(n, 2 n),(n, 3 n)$, and $(n, 4 n)$ reaction. In the usual few group diffusion code the out-scatter cross section is generally used both as a removal term and a source term. However, the standard out-scatter term calculated by COMBINE accounts for the loss of one neutron by an $(n, 2 n),(n, 3 n)$, or $(n, 4 n)$ reaction in a particular broad group. Therefore, when this same term is used as a source term for the next group in a few group diffusion theory calculation of reactivity, it represents only one-half, one-third, or one-foruth of the actual source into that group from the (n,2n), $(n, 3 n)$, or $(n, 4 n)$ transfer reaction. Thus, in the corrected cross-section data, the out-scatter cross section is defined as

$\bar{\Sigma}_{\text {out } G}^{*}=\sum_{G^{\prime}=G+1}^{N O A G}\left(\bar{\sum}_{s 0_{G \rightarrow G^{\prime}}}+\bar{\sum}_{i n_{G \rightarrow G}}+2 \bar{\sum}_{n, 2 n_{G \rightarrow G^{\prime}}}+3 \bar{\sum}_{n, 3 n_{G \rightarrow G^{\prime}}}+4 \bar{\sum}_{n, 3 n_{G \rightarrow G^{\prime}}}\right)+\sum_{G^{\prime}=G-\max u p}^{G-1} \bar{\sum}_{u p_{G \rightarrow G^{\prime}}}$

where now a corrected absorption term is defined as

$$
\sum_{a_{G}}^{*}=\sum_{a_{G}}-\sum_{G^{\prime}=G}^{N O A G} \sum_{n, 2 n_{G \rightarrow G^{\prime}}}-2 \sum_{G^{\prime}=G}^{N O A G} \sum_{n, 3 n_{G \rightarrow G^{\prime}}}-3 \sum_{G^{\prime}=G}^{N O A G} \sum_{n, 4 n_{G \rightarrow G^{\prime}}}
$$

to conserve neutrons. The last term on the right-hand side of Equation (141) reflects the thermal outgoing upscattering.

\subsubsection{Monoenergetic Transport Cross Section}

The monoenergetic transport cross section offers an alternative to the Fick's law definition given by Equation (138) or (139) and has the redeeming feature that is always positive regardless of the shape of

the spectrum. It is the classical value based on its definition from the one-energy transport equation. ${ }^{9}$ For broad group $\mathrm{G}$, the macroscopic value is

$$
\bar{\Sigma}_{t r_{G}}=\bar{\Sigma}_{t_{G}}-\sum_{t_{s 1_{G}}}
$$

Where the cross sections on the right-hand side are given by Equations (134) and (131).

\subsubsection{Average Microscopic Cross Sections}

The average microscopic cross sections are defined in a manner similar to the macroscopic cross sections. The main difference is the use of the microscopic cross section, $\sigma$, in the place of the macroscopic cross section, $\sum$. The one exception is the definition of a microscopic transport cross section. A unique definition of a microscopic transport cross section presents a conceptual difficulty in the same way that a microscopic diffusion coefficient would. The macroscopic values are extensive quantities resulting from some form of Fick's law, and there is no corresponding, physically meaningful relationship for microscopic values. Rather, it has become traditional to define a microscopic transport cross section 
only in the metaphysical sense that "the whole is the sum of its assumed parts"; then from this, the macroscopic diffusion coefficient follows:

$$
\bar{D}_{G}=\frac{1}{3 \sum_{k} N_{k} \bar{\sigma}_{t_{G}}^{k}}=\frac{1}{3 \bar{\Sigma}_{t^{\prime} G}}
$$

This being the case, the only thing required to define the microscopic transport cross section $\bar{\sigma}_{t t_{G}}$ in Equation (141) is a consistency relationship that will yield the proper macroscopic transport cross section for a particular mixture of materials as calculated by Equation (138). Any number of such consistency relationships could be defined and applied, as long as the macroscopic transport cross section in the denominator of Equation (143), computed from microscopic values, gives the same result as Equation (138).

One such equation that will yield the desired results is Equation (139), where $\Sigma^{*}$ becomes the appropriate $\sigma^{k}$ in each term of Equation (135). The broad microscopic transport cross section for the $k^{\text {th }}$ isotope is then given by

$$
\bar{\sigma}_{t_{r_{G}}}^{k}=\frac{\sum_{i=1}^{I+N} \gamma_{i} \sigma_{t_{i}} J_{i} \Delta_{i}-\sum_{G^{\prime}=1}^{N O A G} \sigma_{s 1_{G^{\prime} \rightarrow G}} J_{G^{\prime}}}{J_{G}}
$$

A measure of the "arbitrariness" of this definition of the microscopic transport cross section is that it is entirely possible that the microscopic transport cross section as given by Equation (145) can be negative for one or several materials in the mixture, and yet, when multiplied by Nk and summed over all materials in the mixture, will yield the proper macroscopic transport cross section as given by Equation (138). This situation occurs when the mixture contains large amounts of a strong absorber and the resultant flux and current spectra are very hard. For moderating materials with a small absorption cross section, the second term in the numerator of Equation (145) can then be the leading term due to the high current weights given the transfer cross sections $\sigma_{s 1_{G^{\prime} \rightarrow G}}$ when $\mathrm{G}$ lies in the energy range of depressed flux and current at low energy. The resultant microscopic transport cross sections, even though negative, are consistent in the sense of Equation (143), since "consistency" is all that is required of them in the definition.

The same pathologic situation can occur for cell materials being averaged over the flux and current from another spectral problem. If the spectral problem contained a very strong absorber producing a very hard spectra and the cell material does not contain much absorption, the macroscopic transport cross section from Equation (134) can be negative in low-energy groups. In this case, the negative result could be interpreted as indicating that the homogenization for the cell material was not judiciously chosen.

\subsubsection{Effective Diffusion Theory Constants (Blackness Theory)}

Extensive use is made of diffusion theory in nuclear reactor design analysis; however, inherent in the diffusion theory approximation is its inadequacy in highly absorbing, low scattering regions such as control rods. Because many reactor systems include such regions, the blackness theory was developed to provide a convenient technique for handling these regions without recourse to a higher order transport solution. The approach is to treat the absorbing region as a fictitious diffusing medium of the same thickness but use blackness constants in the diffusion calculation, which yield more correct fluxes than could be obtained with the usual constants. The spectrum code incorporates an option to calculate blackness theory diffusion coefficients and absorption cross sections for use in highly absorbing regions of a system under study. The assumptions made in the development of the formulism used to calculate the 
effective constants are that the absorbing region is a thin slab and the immediately surrounding regions can be adequately handled by diffusion theory methods. These effective constants incorporate factors to account for the mesh structure used to describe the region in a finite difference solution of the diffusion equation. A complete development of this theory can be found in Reference 34 and a review of its use may be found in References 35 and 36.

\subsubsection{Mixed Number Density (MIND) Constants}

An input option allows for the production of mixed number density (MND) constants ${ }^{37}$ for one single broad group in the thermal spectrum. No multiple thermal group MND constants are defined. The constants are defined as

$$
\Sigma_{M N D}=\overline{\sum \mathrm{V}}=\frac{\int_{0}^{E_{I}} \sum(E) \phi_{S P}(E) d E}{\int_{0}^{E_{1}} \frac{1}{\mathrm{v}(E)} \phi_{S P}(E) d E}=\frac{\overline{\left(\sum\right)_{S P}}}{\left(\frac{1}{\mathrm{v}}\right)_{S P}}
$$

where

$$
\begin{aligned}
& \Sigma=\text { the macroscopic absorption or fission cross section } \\
& \phi_{\mathrm{SP}}(\mathrm{E})=\text { thermal flux spectrum solution } \\
& \mathrm{E}_{1}=\text { upper energy of the single MND group } \\
& \mathrm{v}(\mathrm{E})=\text { velocity at energy } \mathrm{E} .
\end{aligned}
$$

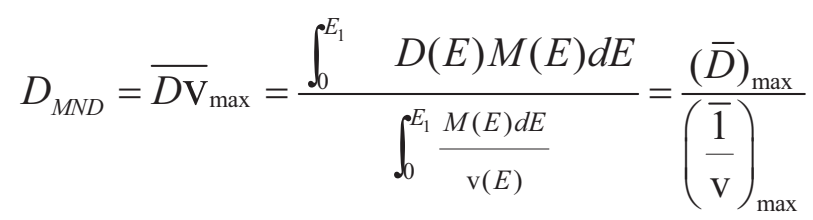

$\sum_{t r}^{M N D}=\frac{1}{3 D_{M N D}}$

$\sigma_{t r}^{M N D}=\left(\frac{\bar{\sigma}_{t r}}{\mathrm{v}_{\max }}\right)=\frac{\int_{0}^{E_{1}} \frac{M(E) d E}{\mathrm{~V}(E)}}{\int_{0}^{E_{1}} \frac{M(E) d E}{\sigma_{t r}(E)}}=\frac{\left(\frac{\overline{1}}{\mathrm{v}}\right)_{\max }}{\left(\frac{1}{\sigma_{t r}}\right)_{\max }}$

where "max" indicates averaging over a Maxwellian spectrum and $\mathrm{M}(\mathrm{E})=$ Maxwellian distribution at temperature T;

$\mathrm{M}(\mathrm{E}) \mathrm{dE} \frac{E[\exp (-E / k T)]}{(k T)^{2}} d E$

where $\mathrm{k}$ is Boltzmann's constant and the average energy $=3 / 2 \mathrm{kT}$ and $\mathrm{T}$ corresponds to the ambient energy of the region. ${ }^{35}$

For each $\sum$ in Equations (146) through (150) there is a corresponding microscopic $\sigma$ calculated for the output files. $\sigma_{\text {tr }}$ replaces D. There are also normalized MND constants defined as: 


$$
\begin{aligned}
D^{N O R M} & =\frac{D_{M N D}}{2.2 \times 10^{5}} \\
\sum_{a}^{N O R M} & =\frac{\sum_{a}^{M N D}}{2.2 \times 10^{5}} \\
\sum_{F}^{N O R M} & =\frac{\sum_{F}^{M N D}}{2.2 \times 10^{5}}
\end{aligned}
$$

Normalized microscopic values are defined as:

$$
\begin{aligned}
\sigma_{t r}^{N O R M} & =\left(2.2 \times 10^{5}\right) \sigma_{t r}^{M N D} \\
\sigma_{a}^{N O R M} & =\frac{\sigma_{a}^{M N D}}{2.2 \times 10^{5}} \\
\sigma_{F}^{N O R M} & =\frac{\sigma_{F}^{M N D}}{2.2 \times 10^{5}}
\end{aligned}
$$

If the "unnormalized MND values" are used in a spatial diffusion calculation, the thermal flux will be values of neutron density. If normalized values are used, the thermal flux will be the $2200 \mathrm{~meters} / \mathrm{sec}$ thermal flux. Summary data printed (and punched) will include MND values of

$$
\Sigma_{t r}, D, \Sigma_{a}, \Sigma_{F}, v \Sigma_{F}, \sigma_{t r}, 1 / 3 \sigma_{t r}, \sigma_{a}, \sigma_{F} \text {, and } v \sigma_{F} \text {. }
$$

\subsubsection{Multiplication Factors}

Multiplication factors $\mathrm{k}_{\text {inf }}$ and $\mathrm{k}_{\text {eff }}$ are calculated for a COMBINE problem and printed after the broad group edits. $\mathrm{k}_{\text {inf }}$ is calculated as

$$
k_{\mathrm{inf}}=\frac{\sum_{G=1}^{G G} v \sum_{f_{G}} \phi_{G}}{\sum_{G=1}^{G G} \sum_{a_{G}} \phi_{G}}
$$

where the numerator is the production rate of fission neutrons over the broad group spectra, the denominator is the absorption rate, and GG is the total number of broad groups. When $B=C$ or $B=-\mathrm{i} C$ with a real constant $C$, then

$$
k_{\text {eff }}=\frac{\sum_{G=1}^{G G} v \sum_{f_{G}} \phi_{G}}{\sum_{G=1}^{G G}\left(\sum_{a_{G}}+D_{G} C_{G}^{2}\right) \phi_{G}}
$$

where $D_{\mathrm{G}}$ is the broad group diffusion coefficient and $C_{G}^{2}$ are the buckling coefficients specified in the floating point input data.

When $B=i C$, 


$$
k_{\text {eff }}=\frac{\sum_{G=1}^{G G} v \sum_{f_{G}} \phi_{G}}{\sum_{G=1}^{G G}\left(\sum_{a_{G}}-D_{G} C_{G}^{2}\right) \phi_{G}}
$$

since there is a negative leakage. In this case $k_{\text {eff }}$ exceeds $k_{\text {inf }}$.

\subsubsection{The Spatial Coalescing Formulas}

The spatial coalescing is carried out by weighting the various cross sections and other neutron group constants as follows. The formulas employed in the INL's internal SCAMP code ${ }^{38}$ were adopted in COMBINE7.1.

Absorption Cross section:

$$
\sum_{R, G}^{a}=\left(\sum_{g \text { in } G} \sum_{r \text { in } R} \sum_{r, g}^{a} \int_{\mathrm{V}_{\mathrm{r}}} \Phi_{g} \mathrm{dv}\right) /\left(\sum_{g \text { in } \mathrm{G}} \sum_{r \text { in } R} \int_{\mathrm{V}_{\mathrm{r}}} \Phi_{g} \mathrm{dv}\right) .
$$

The broad group fission cross section is found in the same manner as the absorption cross section.

$\mathrm{P}_{0}$ Scattering Cross Section:

$$
\Sigma_{R, G \rightarrow G^{\prime}}^{0}=\left(\sum_{g^{\prime} \text { in } G^{\prime}} \sum_{g \text { in } G} \sum_{r \text { in } R} \Sigma_{r, g \rightarrow g^{\prime}}^{0} \int_{\mathrm{V}_{\mathrm{r}}} \Phi_{g} \mathrm{dv}\right) /\left(\sum_{g \text { in } G} \sum_{r \text { in } R} \int_{\mathrm{V}_{\mathrm{r}}} \Phi_{g} \mathrm{dv}\right) .
$$

$\mathrm{P}_{1}$ Scattering Cross Section :

$$
\Sigma_{R, G \rightarrow G^{\prime}}^{1}=\left(\sum_{g^{\prime} \text { in } G^{\prime}} \sum_{g \text { in } G} \sum_{r \text { in } R} \Sigma_{r, g \rightarrow g^{\prime}}^{1} \int_{\mathrm{V}_{\mathrm{r}}} \mathrm{J}_{g} \mathrm{dv}\right) /\left(\sum_{g \text { in } G} \sum_{r \text { in } R} \int_{\mathrm{V}_{\mathrm{r}}} \mathrm{J}_{g} \mathrm{dv}\right) .
$$

The $\mathrm{P}_{2}$ and $\mathrm{P}_{3}$ scattering cross sections are found in the same manner as the $\mathrm{P}_{1}$ scattering cross section but using the $\mathrm{P}_{2}$ and $\mathrm{P}_{3}$ flux moment, respectively. However, the scalar flux is used in the code instead of higher flux moments for the $\mathrm{P}_{0}, \mathrm{P}_{1}$, and $\mathrm{P}_{2}$ scattering cross sections.

Diffusion Coefficient:

$$
\mathrm{D}_{R, G}=\left(\sum_{g \text { in } G} \sum_{r \text { in } R} \mathrm{D}_{r, g} \int_{\mathrm{V}_{\mathrm{r}}}\left|\operatorname{grad} \Phi_{g}\right| \mathrm{dv}\right) /\left(\sum_{g \text { in } G} \sum_{r \text { in } R} \int_{\mathrm{V}_{\mathrm{r}}}\left|\operatorname{grad} \Phi_{g}\right| \mathrm{dv}\right),
$$

where, in each space interval, $\operatorname{grad} \Phi=\left(\Phi_{m+1}-\Phi_{m}\right) /\left(\mathrm{x}_{\mathrm{m}+1}-\mathrm{x}_{\mathrm{m}}\right)$.

Transport Cross Section:

$$
\Sigma_{R, G}^{t r}=1 /\left(3 \mathrm{D}_{R, G}\right) .
$$

Fission Spectrum: 


$$
\chi_{R, G}=\left(\sum_{g \text { in } G} \sum_{r \text { in } R} \chi_{r, g} \int_{\mathrm{v}_{\mathrm{r}}} S_{f} \mathrm{dv}\right) /\left(\sum_{G} \sum_{g \text { in } G} \sum_{r \text { in } R} \chi_{r, g} \int_{\mathrm{v}_{\mathrm{r}}} S_{f} \mathrm{dv}\right),
$$

where $S_{f}^{r}=\frac{1}{\lambda} \sum_{g} v \Sigma_{g}^{f} \Phi_{g}$ and $\lambda=$ eigenvalue.

Neutrons per Fission Times the Fission Cross Section:

$$
v \Sigma_{R, G}^{f}=\frac{\left[\left(\sum_{g \text { in } G} \sum_{r \text { in } R} v \sum_{r, g}^{f} \int_{\mathrm{V}_{\mathrm{r}}} \Phi_{g} \mathrm{dv}\right)\left(\sum_{G} \sum_{g \text { in } G} \sum_{r \text { in } R} \chi_{r, g} \int_{\mathrm{V}_{\mathrm{r}}} S_{f} \mathrm{dv}\right)\right]}{\left[\left(\sum_{g \text { i } G} \sum_{r \text { in } R} \int_{\mathrm{V}_{\mathrm{r}}} \Phi_{g} \mathrm{dv}\right)\left(\sum_{r \text { in } R} \int_{\mathrm{V}_{\mathrm{r}}} S_{f} \mathrm{dv}\right)\right]} .
$$

Parallel Buckling:

$$
\mathrm{B}_{R, G}^{2_{a}}=\left[\sum_{g \text { in } G} \mathrm{C}_{\rho}\left(\mathrm{x}_{u}^{\rho} \mathrm{J}_{u, g}-\mathrm{x}_{l}^{\rho} \mathrm{J}_{l, g}\right)\right] /\left(\mathrm{D}_{R, G} \sum_{g \text { in } G} \sum_{r \text { in } R} \int_{\mathrm{V}_{\mathrm{r}}} \Phi_{g} \mathrm{dv}\right),
$$

where $\rho=0,1$, and 2 for slab, cylindrical, and spherical geometry, respectively, and $C_{0}=1, C_{1}=2 \pi, C_{1}=4 \pi$.

Perpendicular Buckling:

$$
\mathrm{B}_{R, G}^{2_{\perp}}=\left(\sum_{g \text { in } G} \sum_{r \text { in } R} \mathrm{D}_{r, g} \mathrm{~B}_{r, g}^{2_{\perp}} \int_{\mathrm{V}_{\mathrm{r}}} \Phi_{g} \mathrm{dv}\right) /\left(\mathrm{D}_{R, G} \sum_{g \text { in } G} \sum_{r \text { in } R} \int_{\mathrm{V}_{\mathrm{r}}} \Phi_{g} \mathrm{dv}\right) .
$$

Total Cross Section:

$$
\Sigma_{R, G}^{T}=\Sigma_{R, G}^{a}+\mathrm{D}_{\mathrm{R}, \mathrm{G}} \mathrm{B}_{\mathrm{R}, \mathrm{G}}^{2_{\perp}}+\sum_{G^{\prime}} \Sigma_{R, G \rightarrow G^{\prime}}^{0} .
$$

The following formulas were used to calculate the neutron group constants from the transport current and the flux gradient.

Diffusion Coefficient:

$$
\mathrm{D}_{R, G}^{\prime}=\left(\sum_{g \text { in } G} \sum_{r \text { in } R} \int_{\mathrm{V}_{\mathrm{r}}}\left|\mathrm{J}_{g}\right| \mathrm{dv}\right) /\left(\sum_{g \text { in } G} \sum_{r \text { in } R} \int_{\mathrm{V}_{\mathrm{r}}}\left|\operatorname{grad} \Phi_{g}\right| \mathrm{dv}\right) .
$$

Transport Cross Section:

$$
\Sigma_{R, G}^{t r^{\prime}}=1 /\left(3 \mathrm{D}_{R, G}^{\prime}\right)
$$

Parallel Buckling:

$$
\mathrm{B}_{R, G}^{2_{a}^{\prime}}=\mathrm{D}_{R, G} \mathrm{~B}_{R, G}^{2_{a}^{\prime}} / \mathrm{D}_{R, G}^{\prime}
$$

Perpendicular Buckling:

Flux Volume Integrals:

$$
\mathrm{B}_{R, G}^{2_{\perp}^{\prime}}=\mathrm{D}_{R, G} \mathrm{~B}_{R, G}^{2_{\perp}^{\prime}} / \mathrm{D}_{R, G}^{\prime} .
$$




$$
\Phi_{R, G} \mathrm{~V}_{R}=\sum_{g \text { in } G} \sum_{r \text { in } R} \int_{\mathrm{v}_{\mathrm{r}}} \Phi_{g} \mathrm{dv}
$$

Average Fluxes:

$$
\Phi_{R, G}=\sum_{g \text { in } G} \sum_{r \text { in } R} \int_{\mathrm{V}_{\mathrm{r}}} \Phi_{g} \mathrm{dv} / \sum_{r \text { in } R} \int_{\mathrm{V}_{\mathrm{r}}} \Phi_{g} \mathrm{dv}
$$

Average Currents:

$$
\mathrm{J}_{R, G}=\sum_{g \text { in } G} \sum_{r \text { in } R} \int_{\mathrm{V}_{\mathrm{r}}} \mathrm{J}_{g} \mathrm{dv} / \sum_{r \text { in } R} \int_{\mathrm{V}_{\mathrm{r}}} \Phi_{g} \mathrm{dv} .
$$

The disadvantage factor for region $r$ and neutron group $g$ is computed in terms of scalar flux, volume weighted average by the formula

$$
\mathrm{d}_{r, g}=\left[\int_{\mathrm{V}_{\mathrm{r}}} \Phi_{g} \mathrm{dv} / \mathrm{v}_{\mathrm{r}}\right] /\left[\sum_{r} \int_{\mathrm{V}_{\mathrm{r}}} \Phi_{g} \mathrm{dv} / \sum_{r} \mathrm{~V}_{\mathrm{r}}\right] .
$$

The following glossary of terms are used in the above formulas:

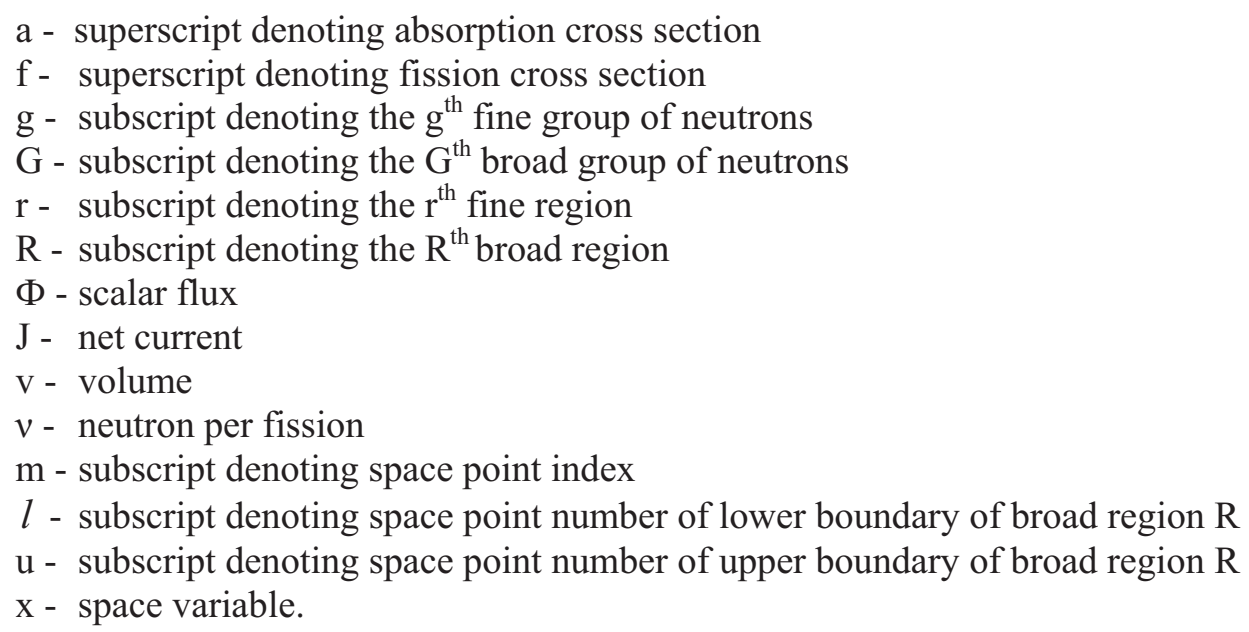




\section{COMBINE7.1 FILES DESCRIPTION}

\begin{tabular}{|c|c|c|c|}
\hline $\begin{array}{c}\text { Logical } \\
\text { Unit }\end{array}$ & Name/status & Format & Usage \\
\hline 401 & matxs.lib & unformatted & Boondarenko based cross section library \\
\hline 402 & reres.lib & formatted & Resolved resonance parameters library \\
\hline 403 & combine.inp & formatted & COMBINE input \\
\hline 404 & combine.out & formatted & COMBINE output \\
\hline 405 & anisn.inp & formatted & ANISN input \\
\hline 406 & anisn.out & formatted & ANISN output \\
\hline 430 & $\begin{array}{l}\text { combine.sig/ } \\
\text { anisnc4.lib }\end{array}$ & formatted & Formerly nt $4=4$ in ANISN \\
\hline 437 & combine.flx & formatted & Flux output \\
\hline 435 & anisnc4.flux & formatted & Formerly 10 in ANISN \\
\hline 445 & anisnc4.pun & formatted & Formerly 7 in ANISN \\
\hline 439 & scratch & unformatted & $\begin{array}{l}\text { temporary self shielded static cross sections, adum, generated in s.r } \\
\text { spectrum }\end{array}$ \\
\hline $411-429$ & scratch & unformatted & $\begin{array}{l}\text { temporary cross section files for cases to be } 1 \text {-D transport (ANISN) } \\
\text { corrected when ioned }=1 \text {, to be used in s.r. xscoal with ioned }=2 \text {. }\end{array}$ \\
\hline 431 & scratch & direct access & total cross sections for Bondarenko self shielding \\
\hline 432 & scratch & unformatted & static (adum) cross section file for region 1 in $\mathrm{ABH}$ \\
\hline 433 & scratch & unformatted & static (adum) cross section file for region 2 in $\mathrm{ABH}$ \\
\hline 434 & scratch & unformatted & static (adum) cross section file for region 3 in $\mathrm{ABH}$ \\
\hline 436 & scratch & unformatted & temporary flux and higher moments \\
\hline 438 & scratch & unformatted & shielded bondarenko cross section file,adum, generated in s.r. bondar \\
\hline 440 & scratch & unformatted & $\begin{array}{l}\text { (sigaz(i),sigfz(i),sigsz(i),amu1(i),i=1,np) for the pricipal resonance absrober } \\
\text { after doppler broadening }\end{array}$ \\
\hline 441 & scratch & unformatted & $\begin{array}{l}\text { temporary }(\operatorname{sigaz}(\mathrm{i}), \operatorname{sigfz}(\mathrm{i}), \operatorname{sigsz}(\mathrm{i}), \mathrm{amu} 1(\mathrm{i}), \mathrm{i}=1, \mathrm{np}) \text { for the pricipal and } \\
\text { admixed resonance absrobers before doppler broadening }\end{array}$ \\
\hline 442 & scratch & unformatted & sa 0 ,ss 0 ,etc., and collision probability file for $\mathrm{ABH}$ \\
\hline 446 & scratch & unformatted & $\begin{array}{l}(\operatorname{sigaz}(\mathrm{i}), \operatorname{sigfz}(\mathrm{i}) \text {,sigsz(i),amu1(i),i=1,np) for the first admixed resonance } \\
\text { absrober after doppler broadening }\end{array}$ \\
\hline 447 & scratch & unformatted & $\begin{array}{l}(\operatorname{sigaz}(i), \operatorname{sigfz}(i), \operatorname{sigsz}(i), \text { amul}(i), i=1, n p) \text { for the second admixed resonance } \\
\text { absrober after broadening }\end{array}$ \\
\hline 448 & scratch & unformatted & $\begin{array}{l}(\operatorname{sigaz}(i), \operatorname{sigfz}(i), \operatorname{sigsz}(i), \text { amul} 1(i), i=1, n p) \text { for the third resonance absrober } \\
\text { after doppler broadening }\end{array}$ \\
\hline 443 & scratch & formatted & $\begin{array}{l}\text { formats and writes broad group output data in s.r. xscoal for post } \\
\text { processing }\end{array}$ \\
\hline 444 & scratch & unformatted & \\
\hline $450-499$ & scratch & unformatted & $\begin{array}{l}\text { thermal matrix cross sections for isotopes used in both sr. bonche and } \\
\text { bondar, is }+449\end{array}$ \\
\hline 407 & scratch & unformatted & Formerly $n t 8=8$ in ANISN \\
\hline 408 & scratch & unformatted & Formerly nt $9=9$ in ANISN \\
\hline 409 & scratch & unformatted & Formerly $n t 1=1$ in ANISN \\
\hline 410 & scratch & unformatted & Formerly $n t t=2$ in ANISN \\
\hline 449 & scratch & unformatted & Formerly 3 in ANISN \\
\hline
\end{tabular}




\section{COMBINE7.1 INPUT DATA FILE ORGANIZATION}

A problem consists of a title record, comment records (optional), data records, and a terminator record. A listing of the records is printed at the beginning of each problem. The order of the title, data, and comment records is unimportant except that only the last title record, or the last data record having a particular data record number, will be used.

When a format error is detected, a line containing a dollar sign (\$) located under the character causing the error and a comment giving the column of the error is printed. An error flag is set such that input processing continues, but the problem will be aborted at the end of input processing.

1. Title Record. A title record must be entered for each problem. A title record is identified by an equal sign (=) as the first nonblank character. The title record is normally placed first in the problem.

2. Comment Records. An asterisk (*) or a dollar sign (\$) appearing as the first nonblank character identifies the record as a comment record. Blank records are treated as comment records. The only processing of comment records is printing of contents. Comment records may be placed anywhere in the input file.

3. Data Records. The data records contain a varying number of fields, which may be integer, floating point, or alphanumeric. Blanks preceding and following fields are ignored.

The first field on a data record is a record number that must be an unsigned integer. If the first field has an error or is not an integer, an error flat is set. Consequently, data on the record are not used and the record will be identified by the record number in the list of unused data records. After each record number and the accompanying data are read, the record number is compared to previously entered record numbers. If a matching record number is found, the data entered on the previous record are replaced by the data on the current record. If the record being processed contains only a record number, the record number and the data entered on the previous record are deleted. If a record causes replacement or deletion of data, a statement is printed indicating that the record is a replacement.

A number field on a data record is started by either a digit ( 0 through 9$)$, a sign $(+$ or -$)$, or a decimal point (.). A comma or a blank (with one exception noted below) terminates a particular number field. The number field has a number part, and optionally, an exponent part. A number field without a decimal point or an exponent is an integer field; a number field with either a decimal point, an exponent, or both is a floating-point field. A floating-point field without a decimal point is assumed to have a decimal point immediately in front of the first digit. The exponent denotes the power of 10 to be applied to the number part of the field. The exponent part has an E and a sign (+ or -) followed by a number giving the power of 10 . These rules for floating point numbers are identical to those for entering data in FORTRAN e or $\mathrm{f}$ format fields except that no blanks (one exception) are allowed between characters. Floating point data punched by FORTRAN programs can be read. To permit reading of floating point data, a blank following an E denoting an exponent is treated as a plus sign. Acceptable ways of entering floating-point numbers, all containing the quantity 12.45 , are illustrated by six fields: $12.45,+12.45,1245+2,1.245+1,1.245 \mathrm{e} 1$, and $1.245 \mathrm{e}+1$.

4. Terminator Records. A record having a period as the first nonblank character is used as a terminator. Most previous input records are erased before the input to the next problem is processed. Therefore, each problem must contain a complete input data file sufficient for that type of problem. Also, the final problem is terminated by a period record.

5. Data Record Summary. A data record contains: the record number and a descriptive title of the data contained on the record; an explanation of any variable data input on this record; and the order of the data on the record, the variable name, and the input data requirements, where applicable. Any variable names beginning with the letters I, J, K, L, M, or N are of type INTEGER. Any variable names starting with another letter are of type REAL. After $\mathrm{N}$ words on a record, default values will be used for the rest. However, data items should not be skipped up to $\mathrm{N}$ words. 


\subsection{Problem/1-D Data Records}

First card:

\begin{tabular}{lll}
\hline Position & \multicolumn{1}{c}{ Name } & \\
\hline Word 1 & NPROB & Number of Problems \\
Word 2 & N1DC & Number of spatial coalescing \\
\hline Word 2 & NPROBID & Problem ID, equivalent to MTLNO as in the 1030101 card \\
\hline
\end{tabular}

This is the first card in combine.inp, which is always required. All words are required.

Second Card:

\begin{tabular}{clll}
\hline Position & \multicolumn{1}{c}{ Name } & & \multicolumn{2}{c}{ Description of Data } \\
\hline Word 1 & IONED & \multicolumn{2}{c}{ Type of problem to be performed } \\
& & 0 & Standard zero-dimensional COMBINE problem. \\
& & 1 & COMBINE/ANISN/COMBINE sequence. \\
& & 2 & COMBINE/ANISN/spatial weighting/COMBINE/ANISN/COMBINE sequence. \\
& & -1 & ANISN only. \\
Word 2 & NSTACK & Total number of stacked cases in a problem. \\
Word 3 & NSTACKID & NSTACK ID, equivalent to MTLNO as in the 1030101 card \\
\hline
\end{tabular}

This is the second card in combine.inp, which is always required. All words are required.

When IONED $=0$, a standard COMBINE only is performed for the zero-dimensional cell calculation and the COMBINE input data as described in the next section follows. PROBID, N1DP and NSTACKID should be set to zero in this case.

When IONED = 1, a standard COMBINE calculation in the full 167 fine-group structure is performed, generating anisnc4.lib. An ANISN input file, 'anisn.inp1' with the igm=167, is required. An ANISN calculation is performed to provide flux and moments back to COMBINE portion. The broad group (as specified in the input) cross section coalescing is performed in COMBINE portion, generating 'combine.sig'. PROBID, N1DP, NSTACKID can be set to zero in this case.

When IONED $=2$, N1DC $>0$, and NPROBID $>0$, a standard COMBINE calculation in the full 167 finegroup structure is performed, generating anisnc4.lib. An ANISN input file, 'anisn.inp1' is required. An ANISN calculation is performed to provide flux and moments back to COMBINE portion. A spatial coalescing is performedformed for the NSTACK cases in the 167 fine-group structure. Additional COMBINE problems are performed and the spatial and energy coalescing are performed over NPROB problems. Additional ANISN inputs are anisn.inp2, anisn.inp3, etc.

When IONED $=-1$, a standard ANISN calculation is performed, no more COMBINE input data is required. The files 'anisn.inp1' and 'anisnc4.lib' are required. 


\subsection{Title Record and Integer Data Records}

\subsubsection{Title Record}

A title record must be present for each problem and is identified by an equal sign $(=)$ as the first character on the record.

\subsubsection{Problem Basic Control Information Record 1010101}

This record is required for each problem. The first four words are always required. ( $\mathrm{N}$ words, $4 \leq \mathrm{N} \leq 9$ )

\begin{tabular}{|c|c|c|}
\hline Position & Name & Description of Data \\
\hline \multirow[t]{5}{*}{ Word 1} & NTYPE & Type of calculation to be performed. \\
\hline & & 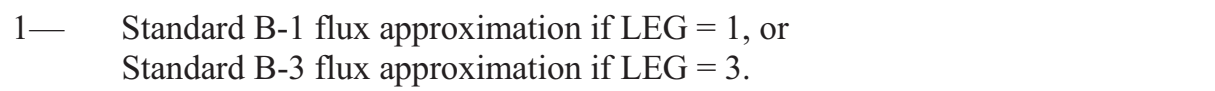 \\
\hline & & Standard P-1 flux approximation if LEG $=1 . \gamma(\mathrm{E})=1.0$ in Equation $(16)$. \\
\hline & & $\begin{array}{l}\text { Cell calculation; fluxes, from the preceding problem will be used for averagin } \\
\text { cross sections. Currents will be recalculated from Equations (32) and }(162) \\
\text { using the P-1 approximation with } \gamma(E)=1.0 \text {. }\end{array}$ \\
\hline & & $\begin{array}{l}\text { Blackness calculation; fluxes from the preceding problem will be used to } \\
\text { obtain effective diffusion theory cross sections. }\end{array}$ \\
\hline \multirow[t]{2}{*}{ Word 2} & LEG & B-1 or P-1 calculation. \\
\hline & & B-3 calculation. \\
\hline Word 3 & NOI & Number of materials in the spectrum, cell or blackness calculation $(1<\mathrm{NOI}<50)$. \\
\hline Word 4 & NOUG & $\begin{array}{l}\text { Total number of broad groups in the spectrum, cell or blackness calculation } \\
(1<\text { NOUG }<167) \text {. }\end{array}$ \\
\hline \multirow[t]{3}{*}{ Word 5} & MICR & $\begin{array}{l}\text { Microscopic cross section option (must be set to } 1 \text { if microscopic cross sections are to } \\
\text { be output to the ASCII cross section file. }\end{array}$ \\
\hline & & $0-\quad$ No average microscopic data desired. (Default) \\
\hline & & Calculate average microscopic cross sections. \\
\hline \multirow[t]{5}{*}{ Word 6} & MSTU & Source option. \\
\hline & & Unit source in fine group MGHS \\
\hline & & Fission rate weighted spectrum will be used. \\
\hline & & $\mathrm{N}-\quad$ Fission spectrum of the material $\mathrm{XX}$ in the $10420 \mathrm{XX}$ card $(\mathrm{N}=\mathrm{XX})$. \\
\hline & & $\begin{array}{l}\text { When rabs in } 1043 \mathrm{XXY} /=0, \mathrm{MSTU} \text { is reset to }-1 \text {. When } \mathrm{NTYPE}=4 \text {, can be set to } 0 \\
\text { (no effect). }\end{array}$ \\
\hline Word 7 & MGHS & $\begin{array}{l}\text { Speifies fine group number for unit source. Rewuired only if a unit source is desired, } \\
\text { i.e., if MSTU }=0 \text {. When NTYPE }=4 \text {, can be set to be } 0 \text { (no effect). }\end{array}$ \\
\hline Word 8 & MAXIT & $\begin{array}{l}\text { Maximum number of iterations when up scattering is present. When } 0 \text {, the code sets } \\
\text { MAXIT = 500. The conversion iteration stops when satisfied either "CONV" or } \\
\text { MAXIT criteria. When there is no up scattering, no iteration is needed. }\end{array}$ \\
\hline \multirow[t]{4}{*}{ Word 9} & ICHET & $\begin{array}{l}\text { No ABH thermal calculation is being requested. No } 2 \mathrm{XXXXXX} \text { records will } \\
\text { be present. (Default) }\end{array}$ \\
\hline & & $\begin{array}{l}\text { An ABH thermal calculation will be performed for rectangular geometry. } \\
\text { Input is on } 2 \text { XXXXXX records. }\end{array}$ \\
\hline & & Cylindrical ABH geometry. \\
\hline & & 3- Spherical ABH geometry. \\
\hline
\end{tabular}




\begin{tabular}{llll}
\hline Position & Name & & \multicolumn{1}{c}{ Description of Data } \\
\hline & & $0-$ & $P_{\mathrm{N}}$ to $S_{\mathrm{N}}$ correction desired.(default). \\
\multirow{3}{*}{ Word 11 } & MAGE & $1-\quad$ No transport correction. \\
& & Age calculation option. \\
& & $0-$ & No age calculation desired. (Default) \\
& & $1-$ & Age calculation desired. \\
Word 12 & MNDFLG & $0-$ & No Mixed number density calculation. (Default) \\
& & $1-$ & Mixed number density calculation. Requires 1490001 record. \\
\hline
\end{tabular}

\subsubsection{Energy Group Structure Record 10102YY}

This record is required for the first problem. YY specifies up to 20 continuation records. ( $\mathrm{N}$ words, $1 \leq \mathrm{N} \leq 167$ )

\begin{tabular}{lcl}
\hline \multicolumn{1}{c}{ Position } & Name & \multicolumn{1}{c}{ Description of Data } \\
\hline $\begin{array}{l}\text { Word 1 to } \\
\text { NGP (I) }\end{array}$ & $\begin{array}{l}\text { Broad energy group structure starting with the upper limit of the first broad group. } \\
(1<\mathrm{I}<167) \text {. See Table 1 following input description. The upper limit of broad group } \\
\text { noumber 1 must be the fine group number 1 (corresponding to 20.0 MeV). } \\
1<\text { number of broad groups }<167\end{array}$ \\
\hline
\end{tabular}

\subsubsection{Resonance Calculation Option Record 102010Y.}

This record is required only if Nordheim resonance calculations are to be performed for resonance materials. If this record is not present, NXSR $=0$ for all materials. Y specifies up to nine continuation records. ( $\mathrm{N}$ words, $1 \leq \mathrm{N} \leq 50$ )

\begin{tabular}{|c|c|c|}
\hline Position & Name & Description of Data \\
\hline \multirow{4}{*}{$\begin{array}{l}\text { Word } 1 \text { to } \\
\text { NOI }\end{array}$} & NXSR (I) & Resonance calculation option $(1<\mathrm{I}<50)$ \\
\hline & & 0-Bondarenko self shielding treatment. \\
\hline & & $\begin{array}{l}\text { 1-Nordheim self shielding treatment (use only for those isotopes with iwr }>0 \text {, see } \\
\text { Table 2). }\end{array}$ \\
\hline & & $\begin{array}{l}\text { 2-Average of Bondarenko and Nordheim treatments. Highly recommended for the } \\
\text { case of LEU. }\end{array}$ \\
\hline
\end{tabular}




\subsubsection{Macroscopic Interface File Option Record 1030101}

This record is only required for each problem for which macroscopic cross-section data are to be output to the interface file, combine.sig. ( $\mathrm{N}$ words, $2 \leq \mathrm{N} \leq 5$ )

\begin{tabular}{|c|c|c|}
\hline Position & Name & $\begin{array}{r}\text { Description of Data } \\
\end{array}$ \\
\hline \multirow[t]{5}{*}{ Word 1} & IPUN & Macroscopic interface file data option. \\
\hline & & 0-No output macroscopic data desired \\
\hline & & 1 - Output the macroscopic cross sections to the ASCII cross-section file. \\
\hline & & $\begin{array}{l}2 \text { - Output the calculated energy-dependent fluxes in neutral ASCII format } \\
\text { (COMBINE.FLX). }\end{array}$ \\
\hline & & 3 - Output both the macroscopic cross sections and the fluxes. \\
\hline \multirow[t]{2}{*}{ Word 2} & MTLNO & Material number used to reference interface file macroscopic cross-section data. \\
\hline & & $\begin{array}{l}\text { If } \mathrm{MTL}(\mathrm{I})<900 \text {, isotope chi values will be added in the ' } 5 \mathrm{~d} \text { ' record of the ISOTXS } \\
\text { format, having ICHI set to } 1 \text {, regardless whether they are all } 0.0 \text {. }\end{array}$ \\
\hline \multirow[t]{5}{*}{ Word 3} & NCOP & Format flag for ASCII cross section output file. \\
\hline & & $0-$ INL format. (Default) \\
\hline & & 1 -ANISN format. \\
\hline & & 2 CCCC ISOTOX format \\
\hline & & $\begin{array}{l}\text { 3-AMPX Working Library format suitable for KENO5A. (not working currently, to } \\
\text { be fixed) }\end{array}$ \\
\hline \multirow[t]{3}{*}{ Word 4} & IAD & $\begin{array}{l}\text { Option parameter that will be placed in columns } 2 \text { and } 3 \text { of the title record } \\
\text { accompanying the macroscopic data to indicate to the diffusion or transport theory } \\
\text { codes how these data are to be used in updating a cross-section library file associated } \\
\text { with these codes. (Used only with INL format). }\end{array}$ \\
\hline & & 0 - Completely replace material MTLNO on the library tape. (Default) \\
\hline & & $\begin{array}{l}1 \text { - Replace only those values on the library tape for material MTLNO which are } \\
\text { entered on the interface records. }\end{array}$ \\
\hline \multirow[t]{3}{*}{ Word 5} & IDIFF & $\begin{array}{l}\text { Option parameter that will be placed in columns } 4 \text { and } 5 \text { of the title record } \\
\text { accompanying the diffusion or transport theory codes indicating whether to use the } \\
\text { values of the diffusion coefficients or transport cross sections. (Used only with INL } \\
\text { format). }\end{array}$ \\
\hline & & 0 - Use the transport cross sections (Monoenergetic definition). (Default) \\
\hline & & 1-Use the diffusion coefficients (Fick's law definition). \\
\hline
\end{tabular}




\subsubsection{Microscopic Interface File Option Record 10302XX}

These records are required only for those materials for which microscopic cross-section data are to be output to the interface file, combine.sig. The material number is indicated by $\mathrm{XX}(1 \leq \mathrm{XX} \leq \mathrm{NOI})(\mathrm{N}$ words, $2 \leq \mathrm{N} \leq 4$ ). Also MICR on record 1010101 must be $=1$.

\begin{tabular}{|c|c|c|}
\hline Position & Name & Description of Data \\
\hline \multirow[t]{3}{*}{ Word 1} & NPUN(I) & Microscopic interface file output option for material I. \\
\hline & & 0 - Do not output microscopic data for this material. \\
\hline & & 1-Output self-shielded microscopic cross-section data. \\
\hline Word 2 & $\operatorname{MTL}(\mathrm{I})$ & $\begin{array}{l}\text { Material number used to reference microscopic cross-section data for material I. This } \\
\text { is used for INL and ISOTXS format output. For ANISN and AMPX format, the } \\
\text { materials are output numbered in ascending order as processed. If MTL(I) }<900 \text {, } \\
\text { isotope chi values will be added in the ' } 5 \mathrm{~d} \text { ' record of the ISOTXS format, having ICHI } \\
\text { set to } 1 \text {, regardless whether they are all } 0.0 \text {. }\end{array}$ \\
\hline \multirow[t]{3}{*}{ Word 3} & $\mathrm{NAD}(\mathrm{I})$ & $\begin{array}{l}\text { Option parameter that will be placed in columns } 2 \text { and } 3 \text { of the title record } \\
\text { accompanying the interface microscopic data for Material I to indicate to the diffusion } \\
\text { or transport theory codes how these data are to be used in updating a cross-section } \\
\text { library file associated with these codes. (Used only with INL format). }\end{array}$ \\
\hline & & 0 - Completely replace material MTL(I) on library file. (Default) \\
\hline & & $\begin{array}{l}1 \text { - Replace only those values on the library file for material MTL(I) that are entered } \\
\text { on the records. }\end{array}$ \\
\hline \multirow[t]{4}{*}{ Word 4} & $\operatorname{NDIFF(I)}$ & $\begin{array}{l}\text { Option parameter that will be placed in columns } 4 \text { and } 5 \text { of the title record } \\
\text { accompanying the interface microscopic data for material I to indicate to the diffusion } \\
\text { or transport theory codes whether to use the values of either the transport cross section } \\
\text { or the values of the quantity } 1 /(3 \sigma \mathrm{tr}) \text {. }\end{array}$ \\
\hline & & (Used only with INL format). \\
\hline & & 0 - Use the transport cross section (monoenergetic definition). (Default) \\
\hline & & 1-Use the quantity $1 /(3 \sigma \operatorname{tr})$ (Fick's law definition). \\
\hline
\end{tabular}




\subsection{Floating Point Data Records \\ 6.3.1 General Floating Point Data Record 1041001}

This record is required for each problem. ( $\mathrm{N}$ words, $1 \leq \mathrm{N} \leq 3$ )

\begin{tabular}{|c|c|c|}
\hline Position & Name & Description of Data \\
\hline \multirow[t]{2}{*}{ Word 1} & BUCK & Constant buckling, cm-2 (B2 value must be nonzero and positive). \\
\hline & & $\begin{array}{l}-1.0-\text { Read } 14700 \mathrm{YY} \text { and } 14710 \mathrm{YY} \text { records for broad group wise buckling and } \\
\text { buck sign. Requires } 14700 \mathrm{YY} \text { and } 14710 \mathrm{YY} \text { records. }\end{array}$ \\
\hline \multirow[t]{4}{*}{ Word 2} & BUCKSIGN & 0.0 - $\operatorname{Exp}(-\mathrm{iBx})$ spatial distribution, i.e., fundamental mode cosine shape. (Default) \\
\hline & & $-1.0-\operatorname{Exp}(-\mathrm{Bx})$ spatial distribution \\
\hline & & $+1.0-\operatorname{Exp}(+\mathrm{Bx})$ spatial distribution \\
\hline & & Applied when BUCK > 0.0 \\
\hline Word 3 & CONV & $\begin{array}{l}\text { Convergence criteria for fluxes and currents or for renormalization factor. (If not } \\
\text { specified or zero, the code sets CONV }=0.00001 \text {.) }\end{array}$ \\
\hline Word 4 & $\mathrm{PF}$ & Packing fraction for the pebble bed application. \\
\hline Word 5 & PEBRAD & Pebble radius $(\mathrm{cm})$ \\
\hline
\end{tabular}

Note: Word 4 and Word 5 are for diffusion coefficient correction to account for the neutron streaming in pebble beds as described in Reference 39.

\subsubsection{Material Specification Record 10420XX}

These records are required for each problem. The material number is indicated by XX $(1 \leq \mathrm{XX} \leq \mathrm{NOI})$. (N words, $3 \leq \mathrm{N} \leq 6)$

\begin{tabular}{|c|c|c|}
\hline Position & Name & Description of Data \\
\hline Word 1 & $\mathrm{BISO}(\mathrm{I})$ & $\begin{array}{l}\text { ENDF material identification number for Ith material. Optionally if negative, print } \\
\text { out the Bondarenko self-shielded fine-group vector cross sections, including } \\
\text { damage, heat, and kerma. }\end{array}$ \\
\hline Word 2 & TISO(I) & Temperature $(\mathrm{K})$ for Ith material. \\
\hline Word 3 & DENS(I) & $\begin{array}{l}\text { Homogeneous atom density } \times 10-24 \text { of the Ith material. May not be zero. Set to a } \\
\text { very small number to force an infinite dilution calculation. }\end{array}$ \\
\hline \multirow[t]{3}{*}{ Word 4} & THERMS(I) & 0.0 - static thermal scattering (no upscattering). (Default) \\
\hline & & 1.0 - free gas thermal scattering (will include upscattering). (see Table 3) \\
\hline & & 2.0-S( $\alpha, \beta)$ thermal scattering (will include upscattering). (see Table 3) \\
\hline Word 5 & EPFISS(I) & Total thermal energy yield/fission $(\mathrm{w} \cdot \mathrm{sec} /$ fiss $) .($ Default $=0.0)$ \\
\hline \multirow[t]{3}{*}{ Word 6} & $\operatorname{SSFF}(\mathrm{I})$ & Self-shielding factor flag for Ith material. \\
\hline & & 0.0 -no input of additional self-shielding factors. (Default) \\
\hline & & $\begin{array}{l}1.0 \text { - additional self-shielding factors input by broad group. The self-shielding } \\
\text { factors are input on records 146XXYY. All cross sections already processed either } \\
\text { by Bondarenko self-shielding treatment or by additional ABH or Nordeim treatment } \\
\text { will be multiplied by these additional self-shielding factors. Care should be taken } \\
\text { when this option is used. }\end{array}$ \\
\hline
\end{tabular}




\subsubsection{Material Resonance Specification Record 1043XXY}

These records are required only for those materials for which a resonance calculation is to be performed. XX indicates the material number where $(1<\mathrm{XX}<\mathrm{NOI})$ and $\mathrm{Y}$ specifies up to 9 continuation records. These records are used for the Nordheim resonance treatment when NXSR $=1.0$ and also for the heterogeneous effect in the Bondarenko treatment when NXSR $=0.0$. ( $\mathrm{N}$ words, $3<\mathrm{N}<23$ )

\begin{tabular}{|c|c|c|}
\hline Position & Name & Description of Data \\
\hline Word 1 & TEMP(I)) & Temperature (K) of absorber lump. \\
\hline \multirow[t]{5}{*}{ Word 2} & GEOM(I) & Geometry of absorber lump. \\
\hline & & 0.0 - Homogeneous medium. DENS(I) is atom density. (see $10420 \mathrm{XX}$ record) \\
\hline & & 1.0 - Slab Geometry heterogeneous. \\
\hline & & 2.0-Cylindrical Geometry heterogeneous. \\
\hline & & 3.0—-Spherical Geometry heterogeneous. \\
\hline Word 3 & DZERO(I) & $\begin{array}{l}\text { Heterogeneous absorber atom density in absorber lump } \times 10^{-24} \text {. May not be zero. } \\
\text { Set to a very small number to force an infinite dilution calculation. }\end{array}$ \\
\hline Word 4 & $\operatorname{ABAR}(\mathrm{I})$ & Lump dimension - radius of cylinder or sphere or thickness of slab in centimeter \\
\hline \multirow[t]{4}{*}{ Word 5} & $\mathrm{CIN}(\mathrm{I})$ & $\begin{array}{l}\text { Dancoff-Ginsburg fuel lump correction factor or option flag. (If no shadowing, } \\
\mathrm{CIN}=0.0 \text {, also CIN is not used if } \operatorname{IGEOM}(\mathrm{I})=0 \text {.) }\end{array}$ \\
\hline & & 0.0 to $1.0-$ Value of the correction factor CIN which is to be read in. \\
\hline & & 2.0—Calculate correction factor (cylinders only). \\
\hline & & $\begin{array}{l}\text { 3.0- Same as for "last material," which required a correction factor. (Caution: } \\
\text { The user should exercise care in using this option.) "Last Material" here means } \\
\text { preceding MATXS ID in the order on library file. }\end{array}$ \\
\hline \multirow[t]{2}{*}{ Word 6} & ELZERO(I) & $\begin{array}{l}\text { Double heterogeneity fuel lump spherical grain average chord length. See } \\
\text { Equation (61) in text. }\end{array}$ \\
\hline & & $\overline{l_{G}}=\frac{4 \mathrm{~V}}{\mathrm{~S}}=\frac{4}{3} \mathrm{r} \mathrm{cm}$ \\
\hline Word 7 & CZERO(I) & $\begin{array}{l}\text { Double heterogeneity fuel lump grain Dancoff factor } \mathrm{C}_{0} \text {. See Equations (43) and } \\
\text { (99) in text. This is independent of CIN(I). }\end{array}$ \\
\hline \multirow[t]{2}{*}{ Word 8} & $\mathrm{~S}(\mathrm{I})$ & 0.0 -Use $\phi_{\text {lump. }}($ Default $)$ \\
\hline & & $\begin{array}{l}1.0-\text { Calculate volume fraction weighted cross sections between those over the } \\
\text { lump flux }\left(\phi_{\text {lump }}\right) \text { and those over the asymptotic flux }\left(\left(\phi_{\text {asym }}\right) \text { outside the lump. }\right.\end{array}$ \\
\hline Word 9 & $\operatorname{AMOD}(\mathrm{I}, 1)$ & First admixed moderator ID. The XX in 10420XX record (not BISO). (Real) \\
\hline Word 10 & $\operatorname{AMOD}(\mathrm{I}, 2)$ & Second admixed moderator ID. The XX in 10420XX record. (Real) \\
\hline Word 11 & $\operatorname{AMOD}(\mathrm{I}, 3)$ & Third admixed moderator ID. The XX in 10420XX record. (Real) \\
\hline Word 12 & $\operatorname{ADENM}(\mathrm{I}, 1)$ & Atomic density of the first admixed moderator, $\operatorname{AMOD}(I, 1)$ \\
\hline Word 13 & $\operatorname{ADENM}(\mathrm{I}, 2)$ & Atomic density of the second admixed moderator, $\operatorname{AMOD}(\mathrm{I}, 2)$ \\
\hline Word 15 & RABS(I,1) & $\begin{array}{l}\text { First admixed resonance absorber ID. The XX in } 10420 \mathrm{XX} \text { record (not BISO). } \\
\text { (Real) }\end{array}$ \\
\hline Word 16 & RABS(I,2) & Second admixed resonance absorber ID. The XX in 10420XX record. (Real) \\
\hline Word 17 & RABS(I,3) & Third admixed resonance absorber ID. The XX in 10420XX record. (Real) \\
\hline Word 18 & ADENABS(I,1) & Atomic density of the first admixed resonance absorber, RABS(I,1). \\
\hline Word 19 & ADENABS(I,2) & Atomic density of the second admixed resonance absorber, RABS(I,2). \\
\hline Word 20 & ADENABS(I,3) & Atomic density of the third admixed resonance absorber, RABS(I,3). \\
\hline Word 21 & RTYPE1(I) & $=0.0$-All waves available in RERES.LIB. (Default) \\
\hline & & ר \\
\hline
\end{tabular}




\begin{tabular}{|c|c|c|}
\hline Position & Name & Description of Data \\
\hline Word 22 & RTYPE2(I) & $\begin{array}{l}=0.0-\text { Use } \mathrm{E}_{\text {high }} \text { as in the RERES.LIB. (Default) } \\
>0.0-\text { Reset } \mathrm{E}_{\text {high }} \text { to RTYPE2(I). }\end{array}$ \\
\hline Word 23 & RTYPE3(I) & $\begin{array}{l}=0.0-\text { Use Elow as in the RERES.LIB. (Default, currently set to } 0.0125 \mathrm{eV} \text { ) } \\
>0.0-\text { Reset } \mathrm{E}_{\text {low }} \text { to RTYPE3(I). }\end{array}$ \\
\hline Word 24 & RTYPE4(I) & $\begin{array}{l}>10.0-\mathrm{NINT}(\mathrm{RTYPE} 4(\mathrm{I})) \text { is the minimum number of mesh points per resonance } \\
\text { (Default, set to } 10 \text { ). Currently, the code sets the maximum number of mesh points } \\
\text { per resonance to } 1400 \text {. }\end{array}$ \\
\hline
\end{tabular}

\subsubsection{Material Dancoff Specification Record 1044XXY}

These records are required for all materials for which a fuel lump Dancoff calculation is to be performed. XX indicates the material number where $1 \leq \mathrm{XX} \leq \mathrm{NOI}$, and $\mathrm{Y}$ specifies up to nine continuation records. ( $\mathrm{N}$ words, $8 \leq \mathrm{N} \leq 12$ )

\begin{tabular}{|c|c|c|}
\hline Position & Name & Description of Data \\
\hline Word 1 & REG(I) & Number of nonabsorber regions in unit cell $(\mathrm{REG}(\mathrm{I})<5)$. \\
\hline \multirow[t]{3}{*}{ Word 2} & $\mathrm{XK}(\mathrm{I})$ & Lattice arrangement of cylindrical pins. \\
\hline & & 1.0 -Rectangular. \\
\hline & & 2.0-Hexagonal. \\
\hline Word 3 & $\operatorname{RADX}(\mathrm{I}, 1)$ & $\begin{array}{l}\text { Outer radius, in centimeters, of first nonabsorber region in unit cell, i.e., region } \\
\text { immediately adjacent to absorber lump. ABAR(I) on record 10430IY is the radius of } \\
\text { the absorber } \mathrm{R}_{0} \text { in Equations (77) and (79). }\end{array}$ \\
\hline Word 4 & $\operatorname{RADX}(\mathrm{I}, 2)$ & Outer radius of second nonabsorber region in unit cell. \\
\hline Word 5 & $\operatorname{RADX}(\mathrm{I}, 3)$ & Outer radius of third nonabsorber region in unit cell. \\
\hline Word 6 & RADX(I,4) & Outer radius of fourth nonabsorber region in unit cell. \\
\hline Word 7 & $\operatorname{RADX}(\mathrm{I}, 5)$ & Outer radius of fifth nonabsorber region in unit cell. \\
\hline Word 8 & $\operatorname{SIG}(1,1)$ & Macroscopic total cross section of first nonabsorber region in unit cell. \\
\hline Word 9 & SIG(I,2) & Macroscopic total cross section of second nonabsorber region in unit cell. \\
\hline Word 10 & SIG(I,3) & Macroscopic total cross section of third nonabsorber region in unit cell. \\
\hline Word 11 & SIG(I,4) & Macroscopic total cross section of fourth nonabsorber region in unit cell. \\
\hline Word 12 & SIG(I,5) & Macroscopic total cross section of fifth nonabsorber region in unit cell. \\
\hline $\begin{array}{l}\text { NOTE: } 1 \\
\text { plus absol } \\
\text { nonabsort } \\
\text { square lat }\end{array}$ & macroscop & $\begin{array}{l}\text { total cross section in each nonabsorber region is the average macroscopic scattering } \\
\text { on in the resolved resonance range } \sum_{i} \text { in Equation (78). The radius of the last } \\
\text { average radius of the unit cell based on the last moderator volume in the hexagonal or }\end{array}$ \\
\hline
\end{tabular}

\subsubsection{Material General Resonance Data Record 10450XX}

These records are required only for those materials for which the user desires to modify the standard resonance calculational procedure. $\mathrm{XX}$ indicates that material number where $1 \leq \mathrm{XX} \leq \mathrm{NOI}$. (N words, 1 $\leq \mathrm{N} \leq 3)$.

\begin{tabular}{lll}
\hline Position & Name & Description of Data \\
\hline Word 1 & THERMUP(I) & $0.0-$ No thermal upscattering in the Nordheim resonance treatment. (Default) \\
& & $1.0-$ Thermal upscattering in the Nordheim resolved resonance treatment.
\end{tabular}




\begin{tabular}{lll}
\hline Position & Name & Description of Data \\
\hline Word 2 & FMULT(I) & $\begin{array}{l}\text { A factor which will multiply the recommended mesh spacing to obtain a coarse or } \\
\text { finer mesh to use in performing the resolved resonance calculation. Default value } \\
\text { is 1.0. If less than 1.0, less meshes are used. If greater than 1.0, more meshes are } \\
\text { used. The maximum number of mesh intervals per resonance, however, is limited } \\
\text { to 904. The minimum is 10. } \\
\text { 0.0-All resonances are used in constructing cross sections. (Default) } \\
\text { X-The number of neighboring resonances in either direction to be considered. } \\
\text { (Not recommended) }\end{array}$ \\
& &
\end{tabular}

\subsubsection{Material Self-Shielding Factor Records (146XXYY)}

These records are required only for those materials if self-shielding factors are to be input, i.e., if $\operatorname{SSFF}(\mathrm{I})=1.0$ for material $\mathrm{XX}$. NOUG values must be input. XX specifies the material number, where 1 $<\mathrm{XX}<\mathrm{NOI}$, and YY specifies up to 20 continuation records.

\begin{tabular}{lll}
\hline Position & Name & \multicolumn{1}{c}{ Description of Data } \\
\hline Word 1 to & SSFBG $(\mathrm{J})$ & $\begin{array}{l}\text { Self-shielding factors for material I (specified by XX) and broad group J, listed in } \\
\text { consecutive order. } \\
\text { NOUG }\end{array}$ \\
& $\begin{array}{l}\text { (1 }<\mathrm{J}<\mathrm{NOUG}) \\
\text { These data are necessary only for those materials that require self-shielding factors. }\end{array}$ \\
& $\begin{array}{l}\text { Input self-shielding factors in the epithermal energy groups for materials with a } \\
\text { resonance range will double self-shield resonance materials which are automatically } \\
\text { self-shielded on records 1044XXY. Input self-shielding factors in this energy range } \\
\text { should be 1.0 or an infinitely dilute resonance calculation should be performed to } \\
\text { prevent double self-shielding of resonances (caveat emptor). }\end{array}$ \\
\hline
\end{tabular}

\subsubsection{Broad Group Buckling Factor Records (14700YY)}

This record is required only if broad group wise buckling is to be input, i.e., if buck $\leq 0.0$ for the problem. NOUG nonzero and positive values must be input. YY specifies up to 20 continuation records. (NOUG words).

\begin{tabular}{lcl}
\hline Position & Name & \multicolumn{1}{c}{ Description of Data } \\
\hline $\begin{array}{l}\text { Word 1 to } \\
\text { NOUG }\end{array}$ & BUCKBG $(\mathrm{J})$ & $\begin{array}{l}\text { Broad group wise buckling corresponding to } 101020 \mathrm{Y} \text { record. }(1 \leq \mathrm{J} \leq \mathrm{NOUG}) \text { (must } \\
\text { be nonzero and positive) }\end{array}$ \\
\hline
\end{tabular}

\subsubsection{Broad Group Buckling Sign Records (14710YY)}

This record is required only if broad group wise buckling is present in $14700 \mathrm{YY}$ record. NOUG values must be input. YY specifies up to 20 continuation records. (NOUG words).

\begin{tabular}{|c|c|c|}
\hline Position & Name & Description of Data \\
\hline \multirow[t]{2}{*}{$\begin{array}{l}\text { Word } 1 \text { to } \\
\text { NOUG }\end{array}$} & $\operatorname{GBSIGN}(\mathrm{J})$ & $\begin{array}{l}\text { Broad group wise buckling sign corresponding to } 14700 \mathrm{YY} \text { record. }(1 \leq \mathrm{J} \leq \\
\text { NOUG). }\end{array}$ \\
\hline & & $\begin{array}{ll}0.0- & \exp (-\mathrm{iBx}) \text { spatial distribution, i.e., fundamental mode cosine shape. } \\
-1.0- & \exp (-\mathrm{Bx}) \text { spatial distribution } \\
+1.0- & \exp (+\mathrm{Bx}) \text { spatial distribution }\end{array}$ \\
\hline
\end{tabular}




\subsubsection{Blackness calculation option Record (1480001)}

This record is required if the blackness calculation has been requested on Record 1010101 $(\mathrm{NTYPE}=6) .(\mathrm{N}$ words, $2 \leq \mathrm{N} \leq 5)$

\begin{tabular}{|c|c|c|}
\hline Position & Name & Description of Data \\
\hline Word 1 & $\mathrm{TH}$ & $\begin{array}{l}\text { Thickness of highly absorbing region, in centimeters, for which a "Blackness theory" } \\
\text { calculation of effective diffusion constants is desired. }\end{array}$ \\
\hline Word 2 & $\mathrm{H}$ & $\begin{array}{l}\text { Width of mesh, in centimeters, used to describe the interior of a highly absorbing } \\
\text { region in a diffusion theory calculation. }\end{array}$ \\
\hline Word 3 & $\mathrm{CB}$ & $\begin{array}{l}\text { A quantity occurring in the blackness theory equations which can be approximated by } \\
3\left(\frac{\sum_{t r}}{\sum_{t}}\right) \\
\text { where } \sum_{t r} \text { and } \sum_{t} \text { represent the transport and total cross sections, respectively, for } \\
\text { the regions immediately surrounding the blackness region. (Default }=3.0 \text {, } \\
\text { assuming } \frac{\sum_{t r} \text { to be unity) }}{\sum_{t}}\end{array}$ \\
\hline Word 4 & AK1 & First blackness coefficient. (usually set to 1.0$).($ Default $=1.0)$ \\
\hline Word 5 & AK2 & Second blackness coefficient. (usually set to 1.0$).($ Default $=1.0)$ \\
\hline
\end{tabular}

\subsubsection{MND calculation option Record (1490001)}

If the MND option has been requested on Card 1010101 (MNDFLG =1), MND constants will be printed out for D, $\Sigma_{\mathrm{a}}, \Sigma_{\mathrm{f}}$ macro and $\sigma_{\mathrm{tr}}, \sigma_{\mathrm{a}}, \sigma_{\mathrm{f}}$ microscopic cross sections. No MND constants will be output in the ASCII file. The other cross sections that are output will not be MND constants. (N words, $2 \leq \mathrm{N} \leq 2)$

\begin{tabular}{llc}
\hline Position & \multicolumn{1}{c}{ Name } & \multicolumn{1}{c}{ Description of Data } \\
\hline Word 1 & $\begin{array}{l}\text { IENDMN } \\
\text { (Integer) }\end{array}$ & Highest fine energy group included in MND calculation. \\
Word 2 & TPMND & Temperature of Maxwellian spectrum for MND calculation. \\
\hline $\begin{array}{l}\text { NOTE: } \\
\text { defined. }\end{array}$ & The & MND option can only be used for a single thermal group. No multithermal group MND option is \\
\hline
\end{tabular}




\subsection{Mixed Integer and Floating Point Data Records}

ABH input data on $2 \mathrm{XXXXXX}$ series records are only required if ICHET $=1$ on the first input record.

\subsubsection{ABH REGION DESCRIPTION RECORDS 2010401}

This record is required for each problem if the $\mathrm{ABH}$ option is to be run. (4 words) See Equations (166) through (175) in the text.

\begin{tabular}{cll}
\hline Position & \multicolumn{1}{c}{ Name } & \multicolumn{1}{c}{ Description of Data } \\
\hline Word 1 & NREG & Number of regions in ABH calculation. NREG must be set to 3.) \\
Word 2 & RAD $(1)$ & Outer radius of region 1 $(\mathrm{cm})$ (half thickness of fuel slab). \\
Word 3 & RAD $(2)$ & Outer radius of region 2 $(\mathrm{cm})$ (cladding). \\
Word 4 & RAD $(3)$ & Outer radius of region 3 $(\mathrm{cm})$ (moderator half thickness in slab geometry). \\
\hline
\end{tabular}

\subsubsection{ABH Material Description for Each Region Record 2015XYY}

These records are required for each problem if the $\mathrm{ABH}$ option is to be run. $\mathrm{X}$ specifies the region number, where $1 \leq \mathrm{X} \leq 3$, and $\mathrm{Y}$ specifies up to 19 continuation records. Up to NOI materials may be specified per region ( $\mathrm{N}$ words, $3 \leq \mathrm{N} \leq 101$ ).

\begin{tabular}{lll}
\hline \multicolumn{1}{c}{ Position } & \multicolumn{1}{c}{ Name } & \multicolumn{1}{c}{ Description of Data } \\
\hline Word 1 & NMAT & Number of materials in region specified by X (integer). \\
Word 2 & $\operatorname{IJSO}(1, \mathrm{X})$ & First material identification number. The XX in 10420XX record. \\
Word 3 & $\mathrm{ADENS}(1, \mathrm{X})$ & Heterogeneous atom density X $10^{-24}$ of the first material in region X. \\
Word 4 & $\operatorname{IJSO}(2, \mathrm{X})$ & Second material identification number. The XX in 10420XX record. \\
Word 5 & $\mathrm{ADENS}(2, \mathrm{X})$ & Heterogeneous atom density X $10^{-24}$ of the second material in region X. \\
\hline NOTE 1: & $\begin{array}{l}\text { In the event the problem requestor wishes to include materials in the ABH calculation with zero atom density to } \\
\text { force the generation of thermal self-shielded microscopic cross sections, an arithmetic indefinite will occur. To } \\
\text { overcome this defect, use a very small atom density, e.g., } 10^{-15} \text { for these materials. }\end{array}$ \\
NOTE 2: & All materials included in the 10420XX cards (NOI materials) should be used in the 20105XY cards. \\
\hline
\end{tabular}




\subsection{Fine Energy Group Structure}

\begin{tabular}{|c|c|c|c|c|c|c|c|}
\hline $\begin{array}{l}\text { Group } \\
\text { Number }\end{array}$ & $\begin{array}{c}\text { Upper } \\
\text { Energy } \\
(\mathrm{eV})\end{array}$ & $\begin{array}{l}\text { Group } \\
\text { Number }\end{array}$ & $\begin{array}{c}\text { Upper } \\
\text { Energy } \\
(\mathrm{eV})\end{array}$ & $\begin{array}{l}\text { Group } \\
\text { Number }\end{array}$ & $\begin{array}{c}\text { Upper } \\
\text { Energy } \\
(\mathrm{eV})\end{array}$ & $\begin{array}{l}\text { Group } \\
\text { Number }\end{array}$ & $\begin{array}{c}\text { Upper } \\
\text { Energy } \\
(\mathrm{eV})\end{array}$ \\
\hline 1 & $2.0000 \mathrm{E}+07$ & 43 & $9.6112 \mathrm{E}+02$ & 85 & $1.1300 \mathrm{E}+00$ & 127 & $3.5000 \mathrm{E}-01$ \\
\hline 2 & $1.6905 \mathrm{E}+07$ & 44 & $7.4852 \mathrm{E}+02$ & 86 & $1.1250 \mathrm{E}+00$ & 128 & $3.4000 \mathrm{E}-01$ \\
\hline 3 & $1.4918 \mathrm{E}+07$ & 45 & $5.8295 \mathrm{E}+02$ & 87 & $1.1100 \mathrm{E}+00$ & 129 & $3.3000 \mathrm{E}-01$ \\
\hline 4 & $1.3499 \mathrm{E}+07$ & 46 & $4.5400 \mathrm{E}+02$ & 88 & $1.0900 \mathrm{E}+00$ & 130 & $3.2000 \mathrm{E}-01$ \\
\hline 5 & $1.1912 \mathrm{E}+07$ & 47 & $3.5358 \mathrm{E}+02$ & 89 & $1.0800 \mathrm{E}+00$ & 131 & $3.1000 \mathrm{E}-01$ \\
\hline 6 & $1.0000 \mathrm{E}+07$ & 48 & $2.7536 \mathrm{E}+02$ & 90 & $1.0700 \mathrm{E}+00$ & 132 & $3.0000 \mathrm{E}-01$ \\
\hline 7 & $7.7880 \mathrm{E}+06$ & 49 & $2.1445 \mathrm{E}+02$ & 91 & $1.0600 \mathrm{E}+00$ & 133 & $2.9000 \mathrm{E}-01$ \\
\hline 8 & $6.0653 \mathrm{E}+06$ & 50 & $1.6702 \mathrm{E}+02$ & 92 & $1.0500 \mathrm{E}+00$ & 134 & $2.8000 \mathrm{E}-01$ \\
\hline 9 & $4.7237 \mathrm{E}+06$ & 51 & $1.3007 \mathrm{E}+02$ & 93 & $1.0250 \mathrm{E}+00$ & 135 & $2.7000 \mathrm{E}-01$ \\
\hline 10 & $3.6788 \mathrm{E}+06$ & 52 & $1.0130 \mathrm{E}+02$ & 94 & $1.0000 \mathrm{E}+00$ & 136 & $2.6000 \mathrm{E}-01$ \\
\hline 11 & $2.8650 \mathrm{E}+06$ & 53 & $7.8893 \mathrm{E}+01$ & 95 & $9.9000 \mathrm{E}-01$ & 137 & $2.5000 \mathrm{E}-01$ \\
\hline 12 & $2.2313 \mathrm{E}+06$ & 54 & $6.1442 \mathrm{E}+01$ & 96 & $9.8000 \mathrm{E}-01$ & 138 & $2.4000 \mathrm{E}-01$ \\
\hline 13 & $1.7377 \mathrm{E}+06$ & 55 & $4.7851 \mathrm{E}+01$ & 97 & $9.7000 \mathrm{E}-01$ & 139 & $2.3000 \mathrm{E}-01$ \\
\hline 14 & $1.3534 \mathrm{E}+06$ & 56 & $3.7267 \mathrm{E}+01$ & 98 & $9.5000 \mathrm{E}-01$ & 140 & $2.2000 \mathrm{E}-01$ \\
\hline 15 & $1.0540 \mathrm{E}+06$ & 57 & $2.9023 \mathrm{E}+01$ & 99 & $9.3000 \mathrm{E}-01$ & 141 & $2.0000 \mathrm{E}-01$ \\
\hline 16 & $8.2085 \mathrm{E}+05$ & 58 & $2.2603 \mathrm{E}+01$ & 100 & $9.1000 \mathrm{E}-01$ & 142 & $1.8000 \mathrm{E}-01$ \\
\hline 17 & $6.3928 \mathrm{E}+05$ & 59 & $1.7603 \mathrm{E}+01$ & 101 & $8.9000 \mathrm{E}-01$ & 143 & $1.6000 \mathrm{E}-01$ \\
\hline 18 & $4.9787 \mathrm{E}+05$ & 60 & $1.3710 \mathrm{E}+01$ & 102 & $8.7600 \mathrm{E}-01$ & 144 & $1.4000 \mathrm{E}-01$ \\
\hline 19 & $3.8774 \mathrm{E}+05$ & 61 & $1.0677 \mathrm{E}+01$ & 103 & $8.5000 \mathrm{E}-01$ & 145 & $1.2000 \mathrm{E}-01$ \\
\hline 20 & $3.0197 \mathrm{E}+05$ & 62 & $8.3153 \mathrm{E}+00$ & 104 & $8.0000 \mathrm{E}-01$ & 146 & $1.0000 \mathrm{E}-01$ \\
\hline 21 & $2.3518 \mathrm{E}+05$ & 63 & $6.4760 \mathrm{E}+00$ & 105 & $7.5000 \mathrm{E}-01$ & 147 & $9.5000 \mathrm{E}-02$ \\
\hline 22 & $1.8316 \mathrm{E}+05$ & 64 & $5.0435 \mathrm{E}+00$ & 106 & $7.0000 \mathrm{E}-01$ & 148 & $9.0000 \mathrm{E}-02$ \\
\hline 23 & $1.4264 \mathrm{E}+05$ & 65 & $3.9279 \mathrm{E}+00$ & 107 & $6.8300 \mathrm{E}-01$ & 149 & $8.5000 \mathrm{E}-02$ \\
\hline 24 & $1.1109 \mathrm{E}+05$ & 66 & $3.0590 \mathrm{E}+00$ & 108 & $6.5000 \mathrm{E}-01$ & 150 & $8.0000 \mathrm{E}-02$ \\
\hline 25 & $8.6517 \mathrm{E}+04$ & 67 & $2.3824 \mathrm{E}+00$ & 109 & $6.2500 \mathrm{E}-01$ & 151 & $7.5000 \mathrm{E}-02$ \\
\hline 26 & $6.7379 \mathrm{E}+04$ & 68 & $2.3300 \mathrm{E}+00$ & 110 & $6.0000 \mathrm{E}-01$ & 152 & $7.0000 \mathrm{E}-02$ \\
\hline 27 & $5.2475 \mathrm{E}+04$ & 69 & $2.2900 \mathrm{E}+00$ & 111 & $5.9000 \mathrm{E}-01$ & 153 & $6.5000 \mathrm{E}-02$ \\
\hline 28 & $4.0868 \mathrm{E}+04$ & 70 & $2.2000 \mathrm{E}+00$ & 112 & $5.7500 \mathrm{E}-01$ & 154 & $6.0000 \mathrm{E}-02$ \\
\hline 29 & $3.1828 \mathrm{E}+04$ & 71 & $2.1000 \mathrm{E}+00$ & 113 & $5.5000 \mathrm{E}-01$ & 155 & $5.0000 \mathrm{E}-02$ \\
\hline 30 & $2.4788 \mathrm{E}+04$ & 72 & $2.0000 \mathrm{E}+00$ & 114 & $5.3200 \mathrm{E}-01$ & 156 & $4.0000 \mathrm{E}-02$ \\
\hline 31 & $1.9305 \mathrm{E}+04$ & 73 & $1.9000 \mathrm{E}+00$ & 115 & $5.0000 \mathrm{E}-01$ & 157 & $3.0000 \mathrm{E}-02$ \\
\hline 32 & $1.5034 \mathrm{E}+04$ & 74 & $1.8600 \mathrm{E}+00$ & 116 & $4.9000 \mathrm{E}-01$ & 158 & $2.5000 \mathrm{E}-02$ \\
\hline 33 & $1.1709 \mathrm{E}+04$ & 75 & $1.7800 \mathrm{E}+00$ & 117 & $4.8000 \mathrm{E}-01$ & 159 & $2.0000 \mathrm{E}-02$ \\
\hline 34 & $9.1188 \mathrm{E}+03$ & 76 & $1.7000 \mathrm{E}+00$ & 118 & $4.7500 \mathrm{E}-01$ & 160 & $1.5000 \mathrm{E}-02$ \\
\hline 35 & $7.1017 \mathrm{E}+03$ & 77 & $1.6000 \mathrm{E}+00$ & 119 & $4.7000 \mathrm{E}-01$ & 161 & $1.0000 \mathrm{E}-02$ \\
\hline 36 & $5.5308 \mathrm{E}+03$ & 78 & $1.5000 \mathrm{E}+00$ & 120 & $4.6000 \mathrm{E}-01$ & 162 & $8.0000 \mathrm{E}-03$ \\
\hline 37 & $4.3074 \mathrm{E}+03$ & 79 & $1.4400 \mathrm{E}+00$ & 121 & $4.5000 \mathrm{E}-01$ & 163 & $7.0000 \mathrm{E}-03$ \\
\hline 38 & $3.3546 \mathrm{E}+03$ & 80 & $1.3500 \mathrm{E}+00$ & 122 & $4.3000 \mathrm{E}-01$ & 164 & $5.0000 \mathrm{E}-03$ \\
\hline 39 & $2.6126 \mathrm{E}+03$ & 81 & $1.3000 \mathrm{E}+00$ & 123 & $4.2000 \mathrm{E}-01$ & 165 & $4.0000 \mathrm{E}-03$ \\
\hline 40 & $2.0347 \mathrm{E}+03$ & 82 & $1.2500 \mathrm{E}+00$ & 124 & $4.1400 \mathrm{E}-01$ & 166 & $2.0000 \mathrm{E}-03$ \\
\hline 41 & $1.5846 \mathrm{E}+03$ & 83 & $1.2000 \mathrm{E}+00$ & 125 & $3.8000 \mathrm{E}-01$ & 167 & $1.0000 \mathrm{E}-03$ \\
\hline 42 & $1.2341 \mathrm{E}+03$ & 84 & $1.1500 \mathrm{E}+00$ & 126 & $3.6000 \mathrm{E}-01$ & & $1.0000 \mathrm{E}-05$ \\
\hline
\end{tabular}




\subsection{Materials in the "MATXS" Fine Group Cross Section Library and the "RERES" Resolved Resonance Library}

\begin{tabular}{|c|c|c|c|c|c|c|c|}
\hline $\begin{array}{l}\text { Element/ } \\
\text { isotope }\end{array}$ & $\begin{array}{c}\text { ENDF/B } \\
\text { Material } \\
\text { ID }\end{array}$ & $\begin{array}{l}\text { Resolved } \\
\text { Resonance } \\
\text { in reres.lib* }\end{array}$ & $\begin{array}{l}\text { Thermal } \\
\text { Scattering }^{* *}\end{array}$ & $\begin{array}{l}\text { Element/ } \\
\text { Isotope }\end{array}$ & $\begin{array}{c}\text { ENDF/B } \\
\text { Material } \\
\text { ID }\end{array}$ & $\begin{array}{l}\text { Resolved } \\
\text { Resonance } \\
\text { in reres.lib }\end{array}$ & $\begin{array}{l}\text { Thermal } \\
\text { Scattering }\end{array}$ \\
\hline H-1 & 125 & 0 & $\mathrm{H}$ in $\mathrm{H}_{2} \mathrm{O}, \mathrm{f}$ & $\mathrm{Ca}-48$ & 2049 & 2 & $\mathrm{f}$ \\
\hline $\mathrm{H}-1$ & 126 & 0 & $\mathrm{H}$ in $\mathrm{ZrH}$ & Sc-45 & 2125 & 2 & $\mathrm{f}$ \\
\hline $\mathrm{H}-1$ & 127 & 0 & $\mathrm{H}$ in $\mathrm{Ch} 2$ & Ti-46 & 2225 & 2 & $\mathrm{f}$ \\
\hline $\mathrm{H}-2$ & 128 & 0 & $\mathrm{D}$ in $\mathrm{D}_{2} \mathrm{O}, \mathrm{f}$ & Ti-47 & 2228 & 2 & $\mathrm{f}$ \\
\hline H-3 & 131 & 0 & $\mathrm{f}$ & Ti-48 & 2231 & 2 & $\mathrm{f}$ \\
\hline $\mathrm{He}-3$ & 225 & 0 & $\mathrm{f}$ & Ti-49 & 2234 & 2 & $\mathrm{f}$ \\
\hline $\mathrm{He}-4$ & 228 & 0 & $\mathrm{f}$ & Ti-50 & 2237 & 2 & $\mathrm{f}$ \\
\hline Li-6 & 325 & 0 & $\mathrm{f}$ & $\mathrm{V}$ & 2300 & 5 & $\mathrm{f}$ \\
\hline Li-7 & 328 & 0 & $\mathrm{f}$ & Cr-50 & 2425 & 3 & $\mathrm{f}$ \\
\hline Be-9 & 425 & 0 & Be-metal & Cr-52 & 2431 & 3 & $\mathrm{f}$ \\
\hline Be-9 & 426 & 0 & $\mathrm{Be}$ in $\mathrm{BeO}$ & Cr-53 & 2434 & 3 & $\mathrm{f}$ \\
\hline B-10 & 525 & 0 & $\mathrm{f}$ & Cr-54 & 2437 & 3 & $\mathrm{f}$ \\
\hline B-11 & 528 & 0 & $\mathrm{f}$ & Mn-55 & 2525 & 2 & $\mathrm{f}$ \\
\hline $\mathrm{C}$ & 600 & 0 & graphite, $\mathrm{f}$ & $\mathrm{Fe}-54$ & 2625 & 3 & $\mathrm{f}$ \\
\hline $\mathrm{N}-14$ & 725 & 0 & $\mathrm{f}$ & $\mathrm{Fe}-56$ & 2631 & 3 & $\mathrm{f}$ \\
\hline $\mathrm{N}-15$ & 728 & 0 & $\mathrm{f}$ & $\mathrm{Fe}-57$ & 2634 & 3 & $\mathrm{f}$ \\
\hline O-16 & 825 & 0 & $\mathrm{f}$ & Fe-58 & 2637 & 3 & $\mathrm{f}$ \\
\hline $\mathrm{O}-16$ & 826 & 0 & $\mathrm{O}$ in $\mathrm{BeO}$ & Co-58 & 2722 & 2 & $\mathrm{f}$ \\
\hline $\mathrm{O}-17$ & 828 & 0 & $\mathrm{f}$ & Co-58m & 2723 & 2 & $\mathrm{f}$ \\
\hline F-19 & 925 & 0 & $\mathrm{f}$ & Co-59 & 2725 & 3 & $\mathrm{f}$ \\
\hline $\mathrm{Na}-22$ & 1122 & 0 & $\mathrm{f}$ & Ni-58 & 2825 & 3 & $\mathrm{f}$ \\
\hline $\mathrm{Na}-23$ & 1125 & 2 & $\mathrm{f}$ & Ni-59 & 2828 & 2 & $\mathrm{f}$ \\
\hline $\mathrm{Mg}-24$ & 1225 & 0 & $\mathrm{f}$ & $\mathrm{Ni}-60$ & 2831 & 3 & $\mathrm{f}$ \\
\hline $\mathrm{Mg}-25$ & 1228 & 0 & $\mathrm{f}$ & $\mathrm{Ni}-61$ & 2834 & 3 & $\mathrm{f}$ \\
\hline Mg-26 & 1231 & 0 & $\mathrm{f}$ & $\mathrm{Ni}-62$ & 2837 & 3 & $\mathrm{f}$ \\
\hline Al-27 & 1325 & 3 & $\mathrm{f}$ & Ni-64 & 2843 & 3 & $\mathrm{f}$ \\
\hline $\mathrm{Si}-28$ & 1425 & 3 & $\mathrm{f}$ & $\mathrm{Cu}-63$ & 2925 & 3 & $\mathrm{f}$ \\
\hline Si-29 & 1428 & 3 & $\mathrm{f}$ & $\mathrm{Cu}-65$ & 2931 & 3 & $\mathrm{f}$ \\
\hline Si-30 & 1431 & 3 & $\mathrm{f}$ & $\mathrm{Zn}$ & 3000 & 5 & $\mathrm{f}$ \\
\hline P-31 & 1525 & 0 & $\mathrm{f}$ & Ga-69 & 3125 & 2 & $\mathrm{f}$ \\
\hline S-32 & 1625 & 2 & $\mathrm{f}$ & Ga-71 & 3131 & 2 & $\mathrm{f}$ \\
\hline S-33 & 1628 & 2 & $\mathrm{f}$ & Ge-70 & 3225 & 2 & $\mathrm{f}$ \\
\hline S-34 & 1631 & 2 & $\mathrm{f}$ & Ge-72 & 3231 & 2 & $\mathrm{f}$ \\
\hline S-36 & 1637 & 0 & $\mathrm{f}$ & Ge-73 & 3234 & 2 & $\mathrm{f}$ \\
\hline Cl-35 & 1725 & 0 & $\mathrm{f}$ & Ge-74 & 3237 & 2 & $\mathrm{f}$ \\
\hline Cl-37 & 1731 & 0 & $\mathrm{f}$ & Ge-76 & 3243 & 2 & $\mathrm{f}$ \\
\hline Ar-36 & 1825 & 2 & $\mathrm{f}$ & As-74 & 3322 & 2 & $\mathrm{f}$ \\
\hline Ar-38 & 1831 & 2 & $\mathrm{f}$ & As-75 & 3325 & 2 & $\mathrm{f}$ \\
\hline Ar-40 & 1837 & 3 & $\mathrm{f}$ & Se-74 & 3425 & 2 & $\mathrm{f}$ \\
\hline K-39 & 1925 & 2 & $\mathrm{f}$ & Se-76 & 3431 & 2 & $\mathrm{f}$ \\
\hline K-40 & 1928 & 0 & $\mathrm{f}$ & Se-77 & 3434 & 2 & $\mathrm{f}$ \\
\hline $\mathrm{K}-41$ & 1931 & 2 & $\mathrm{f}$ & Se-78 & 3437 & 2 & $\mathrm{f}$ \\
\hline $\mathrm{Ca}-40$ & 2025 & 2 & $\mathrm{f}$ & Se-79 & 3440 & 0 & $\mathrm{f}$ \\
\hline $\mathrm{Ca}-42$ & 2031 & 2 & $\mathrm{f}$ & Se- 80 & 3443 & 2 & $f$ \\
\hline $\mathrm{Ca}-43$ & 2034 & 2 & $\mathrm{f}$ & Se- 82 & 3449 & 2 & $\mathrm{f}$ \\
\hline $\mathrm{Ca}-44$ & 2037 & 2 & $\mathrm{f}$ & Br-79 & 3525 & 2 & $\mathrm{f}$ \\
\hline $\mathrm{Ca}-46$ & 2043 & 0 & $\mathrm{f}$ & Br-81 & 3531 & 2 & $\mathrm{f}$ \\
\hline
\end{tabular}




\begin{tabular}{|c|c|c|c|c|c|c|c|}
\hline $\begin{array}{l}\text { Element/ } \\
\text { isotope }\end{array}$ & $\begin{array}{c}\text { ENDF/B } \\
\text { Material } \\
\text { ID }\end{array}$ & $\begin{array}{l}\text { Resolved } \\
\text { Resonance } \\
\text { in reres.lib* }\end{array}$ & $\begin{array}{l}\text { Thermal } \\
\text { Scattering** }\end{array}$ & $\begin{array}{l}\text { Element/ } \\
\text { Isotope }\end{array}$ & $\begin{array}{c}\text { ENDF/B } \\
\text { Material } \\
\text { ID }\end{array}$ & $\begin{array}{l}\text { Resolved } \\
\text { Resonance } \\
\text { in reres.lib }\end{array}$ & $\begin{array}{l}\text { Thermal } \\
\text { Scattering }\end{array}$ \\
\hline Kr-78 & 3625 & 2 & $\mathrm{f}$ & Rh-105 & 4531 & 1 & $\mathrm{f}$ \\
\hline $\mathrm{Kr}-80$ & 3631 & 2 & $\mathrm{f}$ & Pd-102 & 4625 & 2 & $\mathrm{f}$ \\
\hline Kr-82 & 3637 & 0 & $\mathrm{f}$ & Pd-104 & 4631 & 2 & $\mathrm{f}$ \\
\hline $\mathrm{Kr}-83$ & 3640 & 2 & $\mathrm{f}$ & Pd-105 & 4634 & 2 & $\mathrm{f}$ \\
\hline $\mathrm{Kr}-84$ & 3643 & 2 & $\mathrm{f}$ & Pd-106 & 4637 & 2 & $\mathrm{f}$ \\
\hline Kr-85 & 3646 & 2 & $\mathrm{f}$ & Pd-107 & 4640 & 2 & $\mathrm{f}$ \\
\hline Kr-86 & 3649 & 2 & $\mathrm{f}$ & Pd-108 & 4643 & 2 & $\mathrm{f}$ \\
\hline $\mathrm{Rb}-85$ & 3725 & 2 & $\mathrm{f}$ & Pd-110 & 4649 & 2 & $\mathrm{f}$ \\
\hline $\mathrm{Rb}-86$ & 3728 & 2 & $\mathrm{f}$ & Ag-107 & 4725 & 2 & $\mathrm{f}$ \\
\hline $\mathrm{Rb}-87$ & 3731 & 2 & $\mathrm{f}$ & Ag-109 & 4731 & 2 & $\mathrm{f}$ \\
\hline Sr-84 & 3825 & 2 & $\mathrm{f}$ & $\mathrm{Ag}-110 \mathrm{~m}$ & 4735 & 2 & $\mathrm{f}$ \\
\hline Sr-86 & 3831 & 2 & $\mathrm{f}$ & Ag-111 & 4737 & 2 & $\mathrm{f}$ \\
\hline Sr-87 & 3834 & 2 & $\mathrm{f}$ & Cd-106 & 4825 & 2 & $\mathrm{f}$ \\
\hline Sr- 88 & 3837 & 2 & $\mathrm{f}$ & Cd-108 & 4831 & 2 & $\mathrm{f}$ \\
\hline Sr-89 & 3840 & 0 & $\mathrm{f}$ & Cd-110 & 4837 & 2 & $\mathrm{f}$ \\
\hline Sr-90 & 3843 & 0 & $\mathrm{f}$ & Cd-111 & 4840 & 2 & $\mathrm{f}$ \\
\hline Y-89 & 3925 & 2 & $\mathrm{f}$ & Cd-112 & 4843 & 2 & $\mathrm{f}$ \\
\hline Y-90 & 3928 & 2 & $\mathrm{f}$ & Cd-113 & 4846 & 2 & $\mathrm{f}$ \\
\hline Y-91 & 3931 & 0 & $\mathrm{f}$ & Cd-114 & 4849 & 2 & $\mathrm{f}$ \\
\hline $\mathrm{Zr}$ & 4000 & 5 & $\mathrm{Zr}$ in $\mathrm{ZrH}$ & Cd-115m & 4853 & 2 & $\mathrm{f}$ \\
\hline Zr-90 & 4025 & 2 & $\mathrm{f}$ & $\mathrm{Cd}-116$ & 4855 & 2 & $\mathrm{f}$ \\
\hline Zr-91 & 4028 & 2 & $\mathrm{f}$ & In-113 & 4925 & 2 & $\mathrm{f}$ \\
\hline Zr-92 & 4031 & 2 & $\mathrm{f}$ & In-115 & 4931 & 2 & $\mathrm{f}$ \\
\hline Zr-93 & 4034 & 2 & $\mathrm{f}$ & Sn-112 & 5025 & 2 & $\mathrm{f}$ \\
\hline Zr-94 & 4037 & 2 & $\mathrm{f}$ & Sn-113 & 5028 & 2 & $\mathrm{f}$ \\
\hline Zr-95 & 4040 & 0 & $\mathrm{f}$ & Sn-114 & 5031 & 2 & $\mathrm{f}$ \\
\hline Zr-96 & 4043 & 2 & $\mathrm{f}$ & Sn-115 & 5034 & 2 & $\mathrm{f}$ \\
\hline $\mathrm{Nb}-93$ & 4125 & 1 & $\mathrm{f}$ & Sn-116 & 5037 & 2 & $\mathrm{f}$ \\
\hline $\mathrm{Nb}-94$ & 4128 & 2 & $\mathrm{f}$ & Sn-117 & 5040 & 2 & $\mathrm{f}$ \\
\hline $\mathrm{Nb}-95$ & 4131 & 0 & $\mathrm{f}$ & Sn-118 & 5043 & 2 & $\mathrm{f}$ \\
\hline Mo-92 & 4225 & 2 & $\mathrm{f}$ & Sn-119 & 5046 & 2 & $\mathrm{f}$ \\
\hline Mo-94 & 4231 & 2 & $\mathrm{f}$ & Sn-120 & 5049 & 2 & $\mathrm{f}$ \\
\hline Mo-95 & 4234 & 2 & $\mathrm{f}$ & Sn-122 & 5055 & 2 & $\mathrm{f}$ \\
\hline Mo-96 & 4237 & 2 & $\mathrm{f}$ & Sn-123 & 5058 & 0 & $\mathrm{f}$ \\
\hline Mo-97 & 4240 & 2 & $\mathrm{f}$ & Sn-124 & 5061 & 2 & $\mathrm{f}$ \\
\hline Mo-98 & 4243 & 2 & $\mathrm{f}$ & Sn-125 & 5064 & 2 & $\mathrm{f}$ \\
\hline Mo-99 & 4246 & 0 & $\mathrm{f}$ & Sn-126 & 5067 & 0 & $\mathrm{f}$ \\
\hline Mo-100 & 4249 & 2 & $\mathrm{f}$ & Sb-121 & 5125 & 2 & $\mathrm{f}$ \\
\hline Tc-99 & 4325 & 2 & $\mathrm{f}$ & Sb-123 & 5131 & 2 & $\mathrm{f}$ \\
\hline Ru-96 & 4425 & 0 & $\mathrm{f}$ & Sb-124 & 5134 & 0 & $\mathrm{f}$ \\
\hline Ru-98 & 4431 & 0 & $\mathrm{f}$ & Sb-125 & 5137 & 0 & $\mathrm{f}$ \\
\hline Ru-99 & 4434 & 2 & $\mathrm{f}$ & Sb-126 & 5140 & 2 & $\mathrm{f}$ \\
\hline Ru-100 & 4437 & 2 & $\mathrm{f}$ & Te-120 & 5225 & 0 & $\mathrm{f}$ \\
\hline Ru-101 & 4440 & 2 & $\mathrm{f}$ & Te-122 & 5231 & 2 & $\mathrm{f}$ \\
\hline Ru-102 & 4443 & 2 & $\mathrm{f}$ & Te-123 & 5234 & 2 & $\mathrm{f}$ \\
\hline Ru-103 & 4446 & 2 & $\mathrm{f}$ & Te-124 & 5237 & 2 & $\mathrm{f}$ \\
\hline Ru-104 & 4449 & 2 & $\mathrm{f}$ & Te-125 & 5240 & 2 & $\mathrm{f}$ \\
\hline Ru-105 & 4452 & 0 & $\mathrm{f}$ & Te-126 & 5243 & 2 & $\mathrm{f}$ \\
\hline Ru-106 & 4455 & 0 & $\mathrm{f}$ & Te- $127 \mathrm{~m}$ & 5247 & 0 & $\mathrm{f}$ \\
\hline Rh-103 & 4525 & 2 & $\mathrm{f}$ & Te-128 & 5249 & 2 & $\mathrm{f}$ \\
\hline
\end{tabular}




\begin{tabular}{|c|c|c|c|c|c|c|c|}
\hline $\begin{array}{l}\text { Element/ } \\
\text { isotope }\end{array}$ & $\begin{array}{c}\text { ENDF/B } \\
\text { Material } \\
\text { ID }\end{array}$ & $\begin{array}{l}\text { Resolved } \\
\text { Resonance } \\
\text { in reres.lib* }\end{array}$ & $\begin{array}{l}\text { Thermal } \\
\text { Scattering** }\end{array}$ & $\begin{array}{l}\text { Element/ } \\
\text { Isotope }\end{array}$ & $\begin{array}{c}\text { ENDF/B } \\
\text { Material } \\
\text { ID }\end{array}$ & $\begin{array}{l}\text { Resolved } \\
\text { Resonance } \\
\text { in reres.lib }\end{array}$ & $\begin{array}{l}\text { Thermal } \\
\text { Scattering }\end{array}$ \\
\hline Te-129m & 5253 & 0 & $\mathrm{f}$ & Nd-144 & 6031 & 2 & $\mathrm{f}$ \\
\hline Te-130 & 5255 & 2 & $\mathrm{f}$ & Nd-145 & 6034 & 2 & $\mathrm{f}$ \\
\hline Te-132 & 5261 & 2 & $f$ & Nd-146 & 6037 & 2 & $\mathrm{f}$ \\
\hline $\mathrm{I}-127$ & 5325 & 2 & $f$ & Nd-147 & 6040 & 2 & $\mathrm{f}$ \\
\hline I-129 & 5331 & 2 & $\mathrm{f}$ & Nd-148 & 6043 & 2 & $\mathrm{f}$ \\
\hline $\mathrm{I}-130$ & 5334 & 2 & $\mathrm{f}$ & Nd-150 & 6049 & 2 & $\mathrm{f}$ \\
\hline I-131 & 5337 & 0 & $f$ & Pm-147 & 6149 & 2 & $\mathrm{f}$ \\
\hline $\mathrm{I}-135$ & 5349 & 0 & $\mathrm{f}$ & Pm-148 & 6152 & 0 & $\mathrm{f}$ \\
\hline $\mathrm{Xe}-123$ & 5422 & 0 & $\mathrm{f}$ & Pm-149 & 6155 & 0 & $\mathrm{f}$ \\
\hline Xe-124 & 5425 & 2 & $\mathrm{f}$ & Pm-151 & 6161 & 2 & $\mathrm{f}$ \\
\hline $\mathrm{Xe}-126$ & 5431 & 2 & $\mathrm{f}$ & Sm-144 & 6225 & 2 & $\mathrm{f}$ \\
\hline $\mathrm{Xe}-128$ & 5437 & 2 & $\mathrm{f}$ & Sm-147 & 6234 & 2 & $\mathrm{f}$ \\
\hline $\mathrm{Xe}-129$ & 5440 & 2 & $\mathrm{f}$ & Sm-148 & 6237 & 2 & $\mathrm{f}$ \\
\hline $\mathrm{Xe}-130$ & 5443 & 2 & $\mathrm{f}$ & Sm-149 & 6240 & 2 & $\mathrm{f}$ \\
\hline $\mathrm{Xe}-131$ & 5446 & 2 & $\mathrm{f}$ & Sm-150 & 6243 & 2 & $\mathrm{f}$ \\
\hline $\mathrm{Xe}-132$ & 5449 & 2 & $\mathrm{f}$ & Sm-151 & 6246 & 2 & $\mathrm{f}$ \\
\hline $\mathrm{Xe}-133$ & 5452 & 0 & $\mathrm{f}$ & Sm-152 & 6249 & 2 & $\mathrm{f}$ \\
\hline $\mathrm{Xe}-134$ & 5455 & 2 & $\mathrm{f}$ & Sm-153 & 6252 & 2 & $\mathrm{f}$ \\
\hline $\mathrm{Xe}-135$ & 5458 & 1 & $\mathrm{f}$ & Sm-154 & 6255 & 2 & $\mathrm{f}$ \\
\hline $\mathrm{Xe}-136$ & 5461 & 2 & $\mathrm{f}$ & Eu-151 & 6325 & 2 & $\mathrm{f}$ \\
\hline Cs-133 & 5525 & 2 & $\mathrm{f}$ & Eu-152 & 6328 & 2 & $\mathrm{f}$ \\
\hline Cs-134 & 5528 & 2 & $\mathrm{f}$ & Eu-153 & 6331 & 2 & $\mathrm{f}$ \\
\hline Cs-135 & 5531 & 2 & $\mathrm{f}$ & Eu-154 & 6334 & 2 & $\mathrm{f}$ \\
\hline Cs-136 & 5534 & 0 & $\mathrm{f}$ & Eu-155 & 6337 & 2 & $\mathrm{f}$ \\
\hline Cs-137 & 5537 & 0 & $\mathrm{f}$ & Eu-156 & 6340 & 2 & $\mathrm{f}$ \\
\hline Ba-130 & 5625 & 2 & $\mathrm{f}$ & Eu-157 & 6343 & 2 & $\mathrm{f}$ \\
\hline Ba-132 & 5631 & 2 & $\mathrm{f}$ & Gd-152 & 6425 & 3 & $\mathrm{f}$ \\
\hline Ba-133 & 5634 & 2 & $\mathrm{f}$ & Gd-153 & 6428 & 3 & $\mathrm{f}$ \\
\hline Ba-134 & 5637 & 2 & $\mathrm{f}$ & Gd-154 & 6431 & 3 & $\mathrm{f}$ \\
\hline Ba-135 & 5640 & 2 & $\mathrm{f}$ & Gd-155 & 6434 & 3 & $\mathrm{f}$ \\
\hline Ba-136 & 5643 & 2 & $\mathrm{f}$ & Gd-156 & 6437 & 3 & $\mathrm{f}$ \\
\hline Ba-137 & 5646 & 2 & $\mathrm{f}$ & Gd-157 & 6440 & 3 & $\mathrm{f}$ \\
\hline Ba-138 & 5649 & 2 & $\mathrm{f}$ & Gd-158 & 6443 & 3 & $\mathrm{f}$ \\
\hline Ba-140 & 5655 & 2 & $\mathrm{f}$ & Gd-160 & 6449 & 3 & $\mathrm{f}$ \\
\hline La-138 & 5725 & 2 & $\mathrm{f}$ & Tb-159 & 6525 & 2 & $\mathrm{f}$ \\
\hline La-139 & 5728 & 2 & $\mathrm{f}$ & Tb-160 & 6528 & 3 & $\mathrm{f}$ \\
\hline La-140 & 5731 & 2 & $\mathrm{f}$ & Dy-156 & 6625 & 2 & $\mathrm{f}$ \\
\hline Ce-136 & 5825 & 2 & $\mathrm{f}$ & Dy-158 & 6631 & 2 & $\mathrm{f}$ \\
\hline Ce-138 & 5831 & 2 & $\mathrm{f}$ & Dy-160 & 6637 & 2 & $\mathrm{f}$ \\
\hline Ce-139 & 5834 & 2 & $f$ & Dy-161 & 6640 & 2 & $\mathrm{f}$ \\
\hline Ce-140 & 5837 & 2 & $\mathrm{f}$ & Dy-162 & 6643 & 2 & $\mathrm{f}$ \\
\hline Ce-141 & 5840 & 2 & $\mathrm{f}$ & Dy-163 & 6646 & 2 & $\mathrm{f}$ \\
\hline Ce-142 & 5843 & 2 & $\mathrm{f}$ & Dy-164 & 6649 & 2 & $\mathrm{f}$ \\
\hline Ce-143 & 5846 & 2 & $\mathrm{f}$ & Нo-165 & 6725 & 2 & $\mathrm{f}$ \\
\hline Ce-144 & 5849 & 0 & $\mathrm{f}$ & Ho- $166 \mathrm{~m}$ & 6729 & 2 & $\mathrm{f}$ \\
\hline Pr-141 & 5925 & 2 & $\mathrm{f}$ & Er-162 & 6825 & 2 & $\mathrm{f}$ \\
\hline Pr-142 & 5928 & 2 & $\mathrm{f}$ & Er-164 & 6831 & 2 & $\mathrm{f}$ \\
\hline Pr-143 & 5931 & 2 & $\mathrm{f}$ & Er-166 & 6837 & 2 & $\mathrm{f}$ \\
\hline Nd-142 & 6025 & 2 & $\mathrm{f}$ & Er-167 & 6840 & 2 & $\mathrm{f}$ \\
\hline Nd-143 & 6028 & 2 & $\mathrm{f}$ & Er-168 & 6843 & 2 & $\mathrm{f}$ \\
\hline
\end{tabular}




\begin{tabular}{|c|c|c|c|c|c|c|c|}
\hline $\begin{array}{l}\text { Element/ } \\
\text { isotope }\end{array}$ & $\begin{array}{c}\text { ENDF/B } \\
\text { Material } \\
\text { ID }\end{array}$ & $\begin{array}{l}\text { Resolved } \\
\text { Resonance } \\
\text { in reres.lib }\end{array}$ & $\begin{array}{l}\text { Thermal } \\
\text { Scattering** }\end{array}$ & $\begin{array}{l}\text { Element/ } \\
\text { Isotope }\end{array}$ & $\begin{array}{c}\text { ENDF/B } \\
\text { Material } \\
\text { ID }\end{array}$ & $\begin{array}{l}\text { Resolved } \\
\text { Resonance } \\
\text { in reres.lib }\end{array}$ & $\begin{array}{c}\text { Thermal } \\
\text { Scattering }\end{array}$ \\
\hline Er-170 & 6849 & 2 & $\mathrm{f}$ & U-233 & 9222 & 3 & $\mathrm{f}$ \\
\hline Lu-175 & 7125 & 2 & $\mathrm{f}$ & U-234 & 9225 & 2 & $\mathrm{f}$ \\
\hline Lu-176 & 7128 & 2 & $\mathrm{f}$ & U-235 & 9228 & 3 & $\mathrm{f}$ \\
\hline Hf-174 & 7225 & 2 & $\mathrm{f}$ & U-236 & 9231 & 2 & $\mathrm{f}$ \\
\hline Hf-176 & 7231 & 2 & $\mathrm{f}$ & U-237 & 9234 & 1 & $\mathrm{f}$ \\
\hline Hf-177 & 7234 & 2 & $\mathrm{f}$ & U-238 & 9237 & 3 & $\mathrm{f}$ \\
\hline Hf-178 & 7237 & 2 & $\mathrm{f}$ & U-239 & 9240 & 1 & $\mathrm{f}$ \\
\hline Hf-179 & 7240 & 2 & $\mathrm{f}$ & U-240 & 9243 & 1 & $\mathrm{f}$ \\
\hline Hf-180 & 7243 & 2 & $\mathrm{f}$ & U-241 & 9246 & 1 & $\mathrm{f}$ \\
\hline Ta-181 & 7328 & 2 & $\mathrm{f}$ & Np-235 & 9340 & 0 & $\mathrm{f}$ \\
\hline Ta-182 & 7331 & 2 & $\mathrm{f}$ & Np-236 & 9343 & 2 & $\mathrm{f}$ \\
\hline W-182 & 7431 & 2 & $\mathrm{f}$ & Np-237 & 9346 & 2 & $\mathrm{f}$ \\
\hline W-183 & 7434 & 2 & $\mathrm{f}$ & Np-238 & 9349 & 0 & $\mathrm{f}$ \\
\hline W-184 & 7437 & 2 & $\mathrm{f}$ & Np-239 & 9352 & 0 & $\mathrm{f}$ \\
\hline W-186 & 7443 & 2 & $\mathrm{f}$ & $\mathrm{Pu}-236$ & 9428 & 2 & $\mathrm{f}$ \\
\hline Re-185 & 7525 & 2 & $\mathrm{f}$ & Pu-237 & 9431 & 0 & $\mathrm{f}$ \\
\hline Re-187 & 7531 & 2 & $\mathrm{f}$ & Pu-238 & 9434 & 1 & $\mathrm{f}$ \\
\hline Ir-191 & 7725 & 2 & $\mathrm{f}$ & Pu-239 & 9437 & 3 & $\mathrm{f}$ \\
\hline Ir-193 & 7731 & 2 & $\mathrm{f}$ & $\mathrm{Pu}-240$ & 9440 & 2 & $\mathrm{f}$ \\
\hline Au-197 & 7925 & 2 & $\mathrm{f}$ & Pu-241 & 9443 & 3 & $\mathrm{f}$ \\
\hline Hg-196 & 8025 & 2 & $\mathrm{f}$ & Pu-242 & 9446 & 1 & $\mathrm{f}$ \\
\hline Hg-198 & 8031 & 2 & $\mathrm{f}$ & Pu-243 & 9449 & 1 & $\mathrm{f}$ \\
\hline Hg-199 & 8034 & 2 & $\mathrm{f}$ & Pu-244 & 9452 & 1 & $\mathrm{f}$ \\
\hline Hg-200 & 8037 & 2 & $\mathrm{f}$ & Pu-246 & 9458 & 0 & $\mathrm{f}$ \\
\hline Hg-201 & 8040 & 2 & $\mathrm{f}$ & Am-241 & 9543 & 1 & $\mathrm{f}$ \\
\hline Hg-202 & 8043 & 2 & $\mathrm{f}$ & Am-242 & 9546 & 2 & $\mathrm{f}$ \\
\hline Hg-204 & 8049 & 0 & $f$ & Am-42m & 9547 & 2 & $\mathrm{f}$ \\
\hline $\mathrm{Pb}-204$ & 8225 & 2 & $f$ & Am-243 & 9549 & 1 & $\mathrm{f}$ \\
\hline $\mathrm{Pb}-206$ & 8231 & 3 & $\mathrm{f}$ & Am-244 & 9552 & 0 & $\mathrm{f}$ \\
\hline $\mathrm{Pb}-207$ & 8234 & 3 & $\mathrm{f}$ & Am-44m & 9553 & 0 & $\mathrm{f}$ \\
\hline $\mathrm{Pb}-208$ & 8237 & 3 & $f$ & $\mathrm{Cm}-241$ & 9628 & 0 & $\mathrm{f}$ \\
\hline Bi-209 & 8325 & 2 & $\mathrm{f}$ & Cm-242 & 9631 & 1 & $\mathrm{f}$ \\
\hline Ra-223 & 8825 & 0 & $\mathrm{f}$ & Cm-243 & 9634 & 1 & $\mathrm{f}$ \\
\hline Ra-224 & 8828 & 0 & $\mathrm{f}$ & Cm-244 & 9637 & 2 & $\mathrm{f}$ \\
\hline Ra-225 & 8831 & 0 & $\mathrm{f}$ & $\mathrm{Cm}-245$ & 9640 & 2 & $\mathrm{f}$ \\
\hline Ra-226 & 8834 & 2 & $\mathrm{f}$ & Cm-246 & 9643 & 2 & $\mathrm{f}$ \\
\hline Ac-225 & 8925 & 0 & $\mathrm{f}$ & Cm-247 & 9646 & 1 & $\mathrm{f}$ \\
\hline Ac-226 & 8928 & 0 & $\mathrm{f}$ & Cm-248 & 9649 & 1 & $\mathrm{f}$ \\
\hline Ac-227 & 8931 & 0 & $\mathrm{f}$ & Cm-249 & 9652 & 2 & $\mathrm{f}$ \\
\hline Th-227 & 9025 & 0 & $\mathrm{f}$ & $\mathrm{Cm}-250$ & 9655 & 2 & $\mathrm{f}$ \\
\hline Th-228 & 9028 & 2 & $\mathrm{f}$ & Bk-249 & 9752 & 2 & $\mathrm{f}$ \\
\hline Th-229 & 9031 & 2 & $\mathrm{f}$ & Bk-250 & 9755 & 2 & $\mathrm{f}$ \\
\hline Th-230 & 9034 & 1 & $\mathrm{f}$ & Cf-249 & 9852 & 2 & $\mathrm{f}$ \\
\hline Th-232 & 9040 & 3 & $\mathrm{f}$ & Cf-250 & 9855 & 1 & $\mathrm{f}$ \\
\hline Th-233 & 9043 & 0 & $\mathrm{f}$ & Cf-251 & 9858 & 1 & $\mathrm{f}$ \\
\hline Th-234 & 9046 & 0 & $\mathrm{f}$ & Cf-252 & 9861 & 1 & $\mathrm{f}$ \\
\hline Pa-231 & 9131 & 3 & $\mathrm{f}$ & Cf-253 & 9864 & 1 & $\mathrm{f}$ \\
\hline Pa-232 & 9134 & 2 & $\mathrm{f}$ & Cf-254 & 9867 & 0 & $\mathrm{f}$ \\
\hline Pa-233 & 9137 & 3 & $\mathrm{f}$ & Es-253 & 9913 & 1 & $\mathrm{f}$ \\
\hline U-232 & 9219 & 3 & $\mathrm{f}$ & Es-254 & 9914 & 0 & $\mathrm{f}$ \\
\hline
\end{tabular}




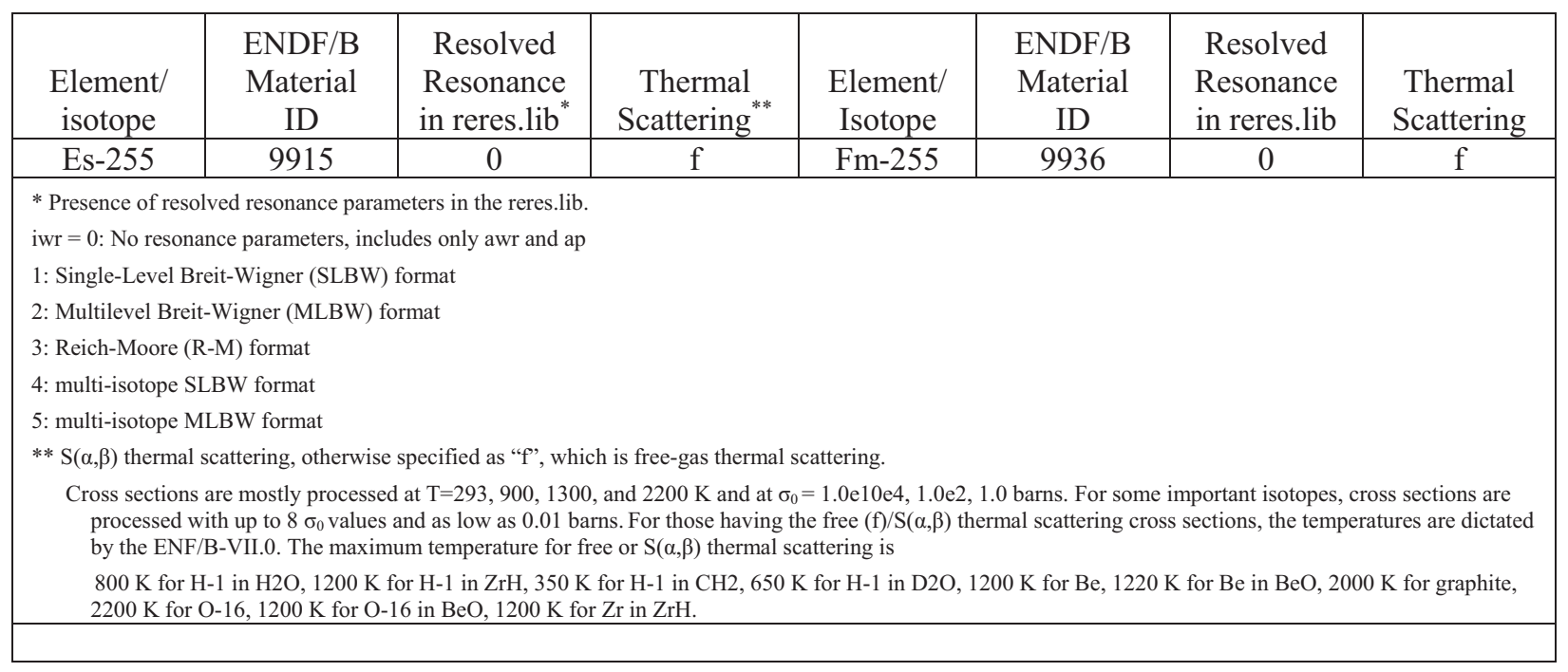




\subsection{MATXS.LIB File Description}

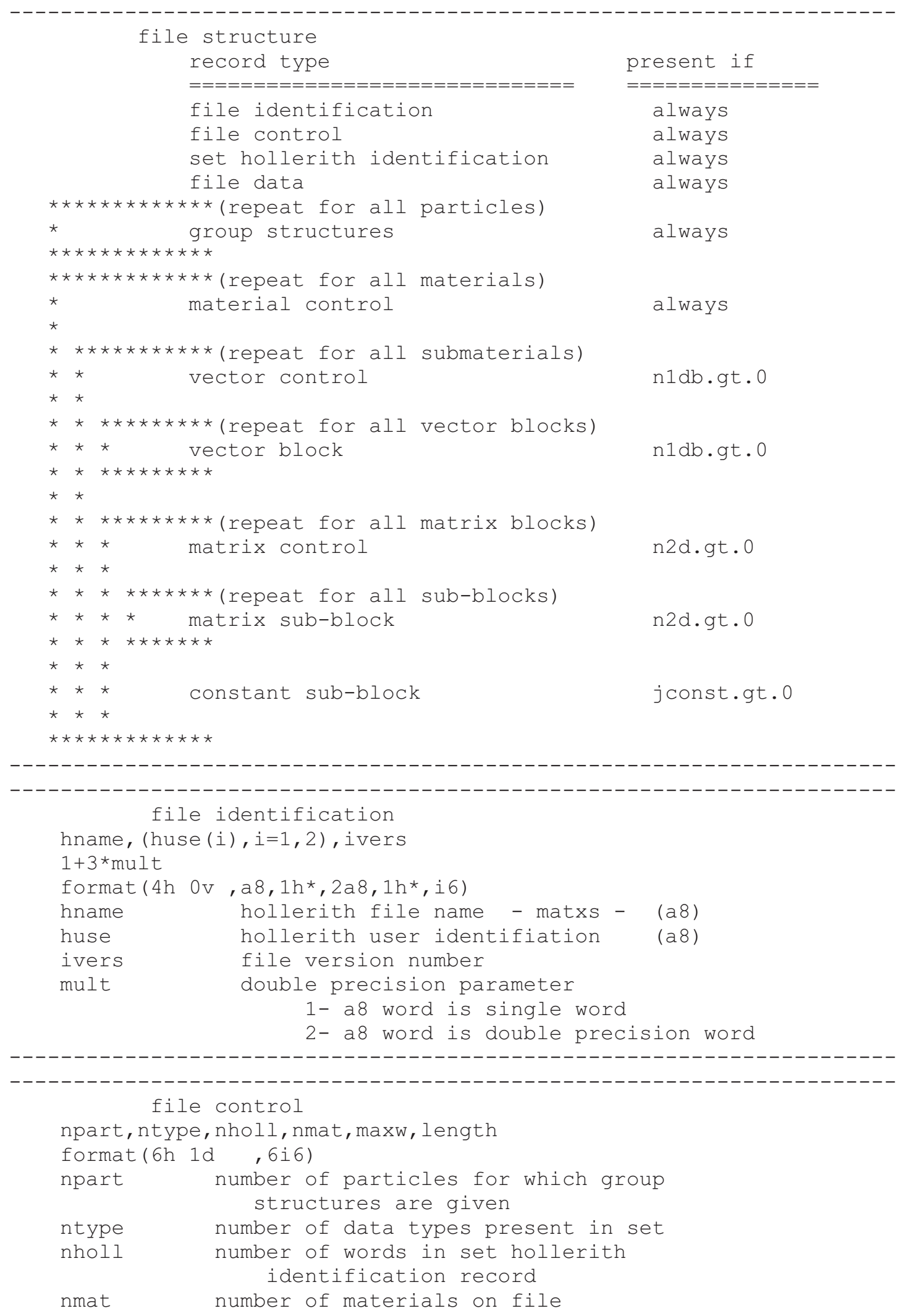




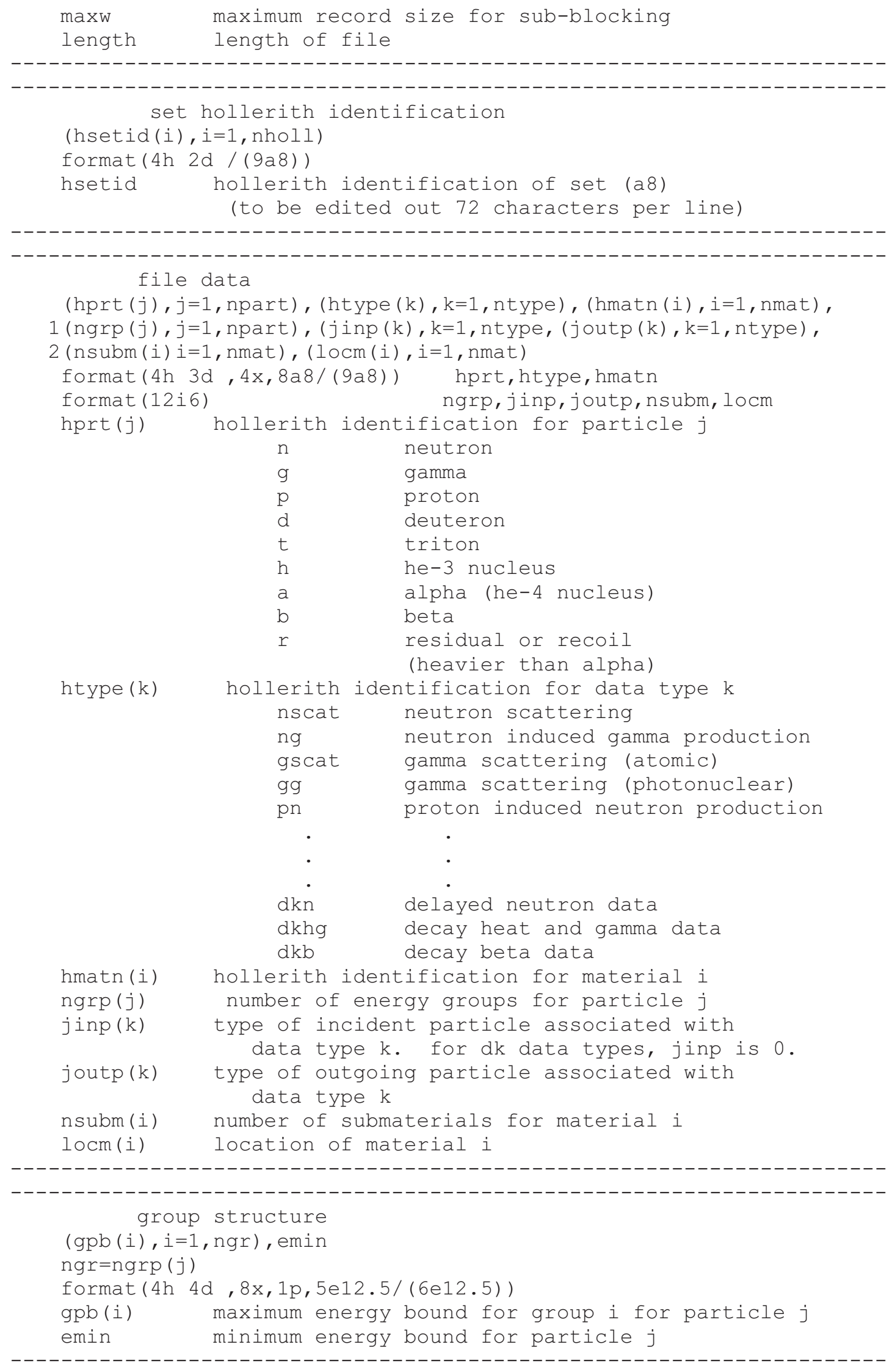




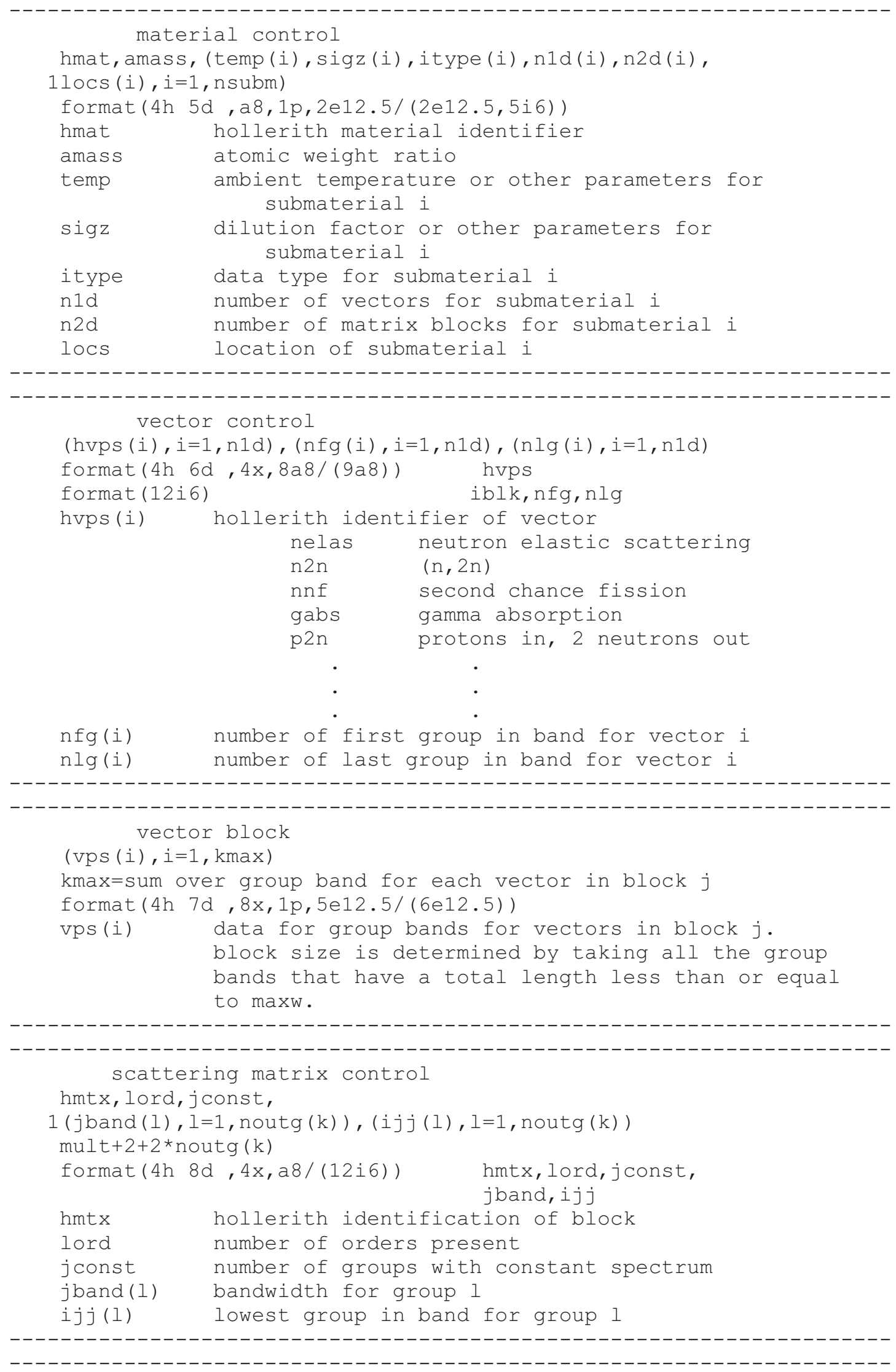




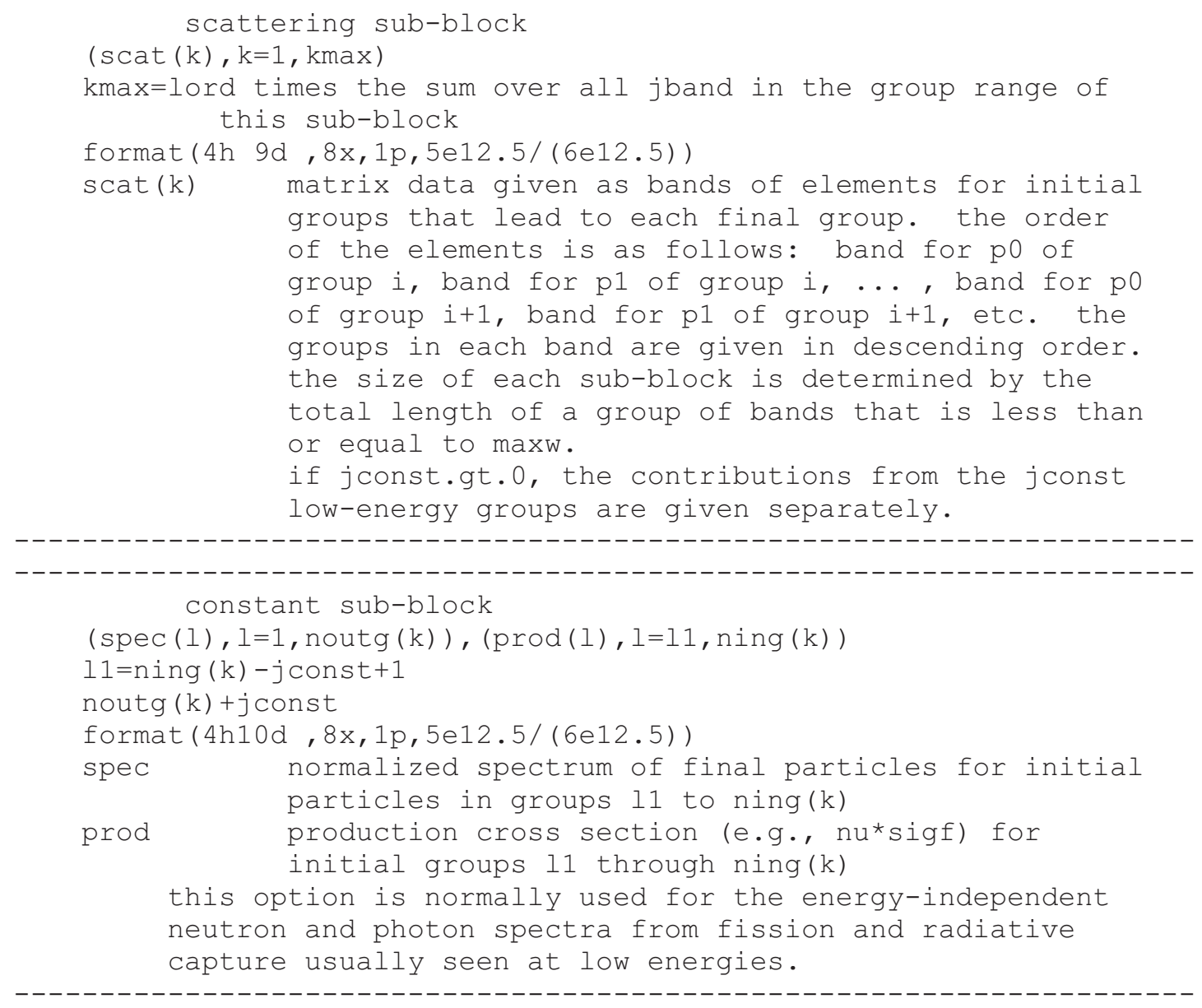




\subsection{Description of Resolved Resonance Library (reres.lib) Format}

Card: title

For a material

Card: aid,iwr,awr,ap,elow,ehigh,nernis,mat (A10,I1,4E11.6,I11,I4)

If $1 \leq \mathrm{iwr} \leq 3$

For $\mathrm{j}=1$,nernis

Card: elt,eht,spi,ap,nls,naps,mat (4E11.6,2I11,I4)

For $1=1, n l s$

Card: nrj,qx,lrx,mat (I11,E11.6,I11,33X,I4)

For $\mathrm{i}=1$, nrj

Card: er,aj,gn,gg,gf,gt,mat (6E11.6,I4)

If $i w r \geq 4$

Card: elt,eht,mat (2E11.6,44X,I4)

For $\mathrm{j}=1$,nernis

Card: awri,abn,naps,spi,ap,nls,mat (2E11.6,I11,2E11.6,I11,I4)

For $1=1, \max (\mathrm{nls})$

Card: nrj,qx,lrx,mat (I11,E11.6,I11,33X,I4)

For $\mathrm{i}=1, \mathrm{nrj}$

Card: igf,er,aj,gn,gg,gt,mat (I11,5E11.6,I4)

Repeat for materials

aid material identification.

iwr 1 for sigle-level Breit-Wigner (SLBW), 2 for multilevel Breit-Wigner (MLBW), 3 for Reich-Moore, 4 for multiisotope SLBW, 5 for multiisotope MLBW.

awr ratio of the material mass to that of a neutron.

ap scattering radius in units of $10^{-12} \mathrm{~cm}$.

elow lower limit for the whole resolved resonance energy range (same as in ENDF/B-VII.0).

ehigh upper limit for the whole resolved resonance energy range (same as in ENDFB-VII.0.

nernis* number of energy ranges for iwr $=1,2,3$ or number of isotopes for iwr $=4,5$.

mat ENDF/B-VII material identification number.

elt lower limit for an energy range (adjusted for COMBINE7, differing from elow: see reres.lib).

eht upper limit for an energy range.

nls number of $l$-values (neutron orbital angular momentum).

naps flag controlling the use of the two radii.

nrj number of resolved resonances for a given l-value.

qx Q-value to be added to the incident particle's center of-mass energy to determine the channel energy.

lrx flag indicating whether this energy range contains a competitive width.

er resonance energy.

aj floating point value of $\mathrm{J}$ (the spin, or total angular momentum of the resonance).

gn neutron width, $\Gamma_{\mathrm{n}}$, evaluated at the resonance energy, er.

gg radiation width,,$\Gamma_{\mathrm{g}}$

gf fission width,,$\Gamma_{\mathrm{f}}\left(\Gamma_{\mathrm{fa}}\right.$ for R-M $)$.

gt resonance total width $\left(\Gamma_{\mathrm{fb}}\right.$ for $\left.\mathrm{R}-\mathrm{M}\right)$.

awri ratio of the mass of a particular isotope to that of a neutron

abn the abundance of an isotope in the material

igf isotope index

* All have only one energy range except Pu-239 which has three energy ranges. 


\subsection{Cross section and constants internal record}

The data in the internal master cross section library consist of alphanumeric, integer, and floating point records. The alphanumeric records are used for identification purposes, the integer data for the most part indicate the number and types of records to be read, and the floating point records make up the actual cross-section data.

The initial data records on the tape are used for identification and to indicate the number of materails, fission spectra and energy groups in the library. Subsequent records describe the cross-section data, with similar types of data records being repeated for each material until all materials have been described. The materails are always arranged in the library so that the identification numbers are in ascending order. The cross-section data records I the library have been condensed as much as feasible to reduce the processing time. Therefore, the length and number of data records present for a particular material depend on the amount of data required to describe the pertinent cross sections. The minimum number of records in the library for any material is two and the maximum is four.

A detailed description of the arrangement of the data within the library, including the mnemonic names and meanings of the data in the order of their occurrences in the library is found below. The cross-section data records for each material are arranged as shown below and are repeated for each material.

\subsubsection{First Data Record for Material k}

\footnotetext{
Mnemonic

Name Description or Function of Data

BISO(IS) Material number

LTOT Integer indicating the total number of words of data contained in the cross-section vector ADUM

IWA Absorption reaction flag.

If IWA $=1$, absorption cross sections are present.

IWF Fission reaction flag.

If IWF $=1$, fission cross sections and the values of the average number of neutrons per fission are present. If IWA $=0$, IWA is ignored.

IWS $\quad \Rightarrow$ No elastic scattering transfer matrices present

1 => Elastic scattering transfer matrices present

LOL(L) Number of cross-section values in List $\mathrm{L}$.

$\mathrm{L}=1 \Rightarrow$ inelastic transfer matrix is present if $|\mathrm{LOL} 1|=1$.

$\mathrm{L}=2 \Rightarrow \mathrm{N}, 2 \mathrm{~N}$ transfer matrix is present if $|\mathrm{LOL} 2|=1$.

$\mathrm{L}=3 \Rightarrow \mathrm{P}_{0}$ and $\mathrm{P}_{1}$ elastic transfer matrix is present if $|\mathrm{LOL} 3|=1$.

$\mathrm{L}=4 \Rightarrow \mathrm{P}_{2}$ and $\mathrm{P}_{3}$ elastic transfer matrix is present if $|\mathrm{LOL} 4|=1$.

$\mathrm{L}=5 \Rightarrow \mathrm{P}_{0}, \mathrm{~N}, 3 \mathrm{~N}$ transfer matrix is present if $|\mathrm{LOL} 5|=1$.

$\mathrm{L}=6 \Rightarrow \mathrm{P}_{0}, \mathrm{~N}, 4 \mathrm{~N}$ transfer matrix is present if $|\mathrm{LOL} 6|=1$.

NOTE: $L O L(1)$ and LOL(2) negative implies $P_{1}$ inelastic or $P_{l}, N, 2 N$ matrices are also present in addition to $P_{0}$ matrices. In either case, $P_{1}$ matrix follows directly behind the $P_{0}$ matrix. The length of either matrix is $/ L O L(1) /$

or

$|\mathrm{LOL}(2)|$. LA and LD vectors apply to both $P_{0}$ and $P_{1}$ matrices in both cases. Only $P_{0}, N, 3 N$ and $N, 4 N$ cross sections are allowed.
}

LA(L) Number of fine groups scattered from in list L, starting with the first fine group, i.e., number of the highest fine group for which scattering reaction of type I is present (threshold group). 


\begin{tabular}{ll}
\multicolumn{1}{c}{$\begin{array}{c}\text { Mnemonic } \\
\text { Name }\end{array}$} & \multicolumn{1}{c}{ Description or Function of Data } \\
\hline $\mathrm{LD}(\mathrm{L})$ & $\begin{array}{l}\text { Maximum number of fine groups scattered to in list L, i.e., the maximum number of groups } \\
\text { downscattered (this value is constant for all groups in which a scattering reaction is tabulated } \\
\text { for a particular material). }\end{array}$ \\
AW(IS) & The atomic weight ratio of this material. \\
\hline
\end{tabular}

\subsubsection{Second Data Record for Material k}

\begin{tabular}{|c|c|}
\hline $\begin{array}{l}\text { Mnemonic } \\
\text { Name }\end{array}$ & Description or Function of Data \\
\hline \multirow[t]{15}{*}{$\begin{array}{l}\text { ADUM(I) } \\
(\mathrm{I}=1, \mathrm{LTOT})\end{array}$} & $\begin{array}{l}\text { Vector containing the complete set of microscopic cross sections and values of neutrons } \\
\text { produced per fission for material } \mathrm{k} \text {. All scattering matrices are in this vector. }\end{array}$ \\
\hline & The cross section values, if present, are contained in the vector in the following order: \\
\hline & $\begin{array}{l}\text { If IWA }=1 \text {, the first MAXG (the number of groups used) values are the absorption cross } \\
\text { sections starting with group } 1 .\end{array}$ \\
\hline & $\begin{array}{l}\text { If } I W A=1 \text {, and } I W F=1 \text {, the next } M A X G \text { values are the fission cross sections followed by } \\
\text { MAXG values of neutrons produced per fission. }\end{array}$ \\
\hline & $\begin{array}{l}\text { If } \mathrm{IWF}=1 \text {, the next MAXG values are the fluxes from MATXS.LIB followed by MAXG chi } \\
\text { values (fission spectrum) for this material. }\end{array}$ \\
\hline & $\begin{array}{l}\text { If }|\mathrm{LOL} 1|=1 \text {, the next } \mathrm{LOL}(1) \text { values are the } \mathrm{P} 0 \text { inelastic cross sections for transfer from } \\
\text { group I to group } \mathrm{J} \text {. The list starts with } \mathrm{I}=1 \text { and contains the values for } \mathrm{J} \text { ranging from } 1 \text { to } \\
\mathrm{LD}(1)+1 \text {. Then the values for } \mathrm{I}=2 \text { with } \mathrm{J} \text { ranging from } 2 \text { to } \mathrm{LD}(1)+2 \text { are next, etc. until } \mathrm{I}= \\
\mathrm{LA}(1) \text {. }\end{array}$ \\
\hline & There are $\mathrm{LD}(1)+1$ values listed for each $\mathrm{I}$, as long as $\mathrm{LD}(1)+\mathrm{I} \leq \mathrm{MAXG}$. When \\
\hline & $\mathrm{LD}(1)+\mathrm{I}>\mathrm{MAXG}$ then only MAXG + 1 - I values are listed for I. \\
\hline & $\begin{array}{l}\text { If } \operatorname{LOL} 1=-1 \text {, the next } \operatorname{LOL}(1) \text { values are the P1 inelastic cross sections for transfer from } \\
\text { group I to group J. The structure of this list is identical to } 4 \text {. }\end{array}$ \\
\hline & $\begin{array}{l}\text { If }|\operatorname{LOL} 2|=1 \text {, next } \operatorname{LOL}(2) \text { values are the } \mathrm{P} 0(\mathrm{n}, 2 \mathrm{n}) \text { cross sections for transfer from group I } \\
\text { to group } \mathrm{J} \text {. The list is ordered as above for the inelastic data. }\end{array}$ \\
\hline & $\begin{array}{l}\text { If LOL } 2=-1 \text {, the next } \operatorname{LOL}(2) \text { values are the P1 }(n, 2 n) \text { cross sections for transfer from group } \\
\text { I to group J. The structure of this list is identical to } 6 \text {. }\end{array}$ \\
\hline & $\begin{array}{l}\text { If LOL } 3=1 \text {, the next } \operatorname{LOL}(3) \text { values are the isotropic elastic scattering cross sections for } \\
\text { transfer from group I to group } \mathrm{J} \text { followed by the same number of values for the linearly } \\
\text { anisotropic component of the elastic scattering cross sections from group I to group J. }\end{array}$ \\
\hline & $\begin{array}{l}\text { If LOL } 4=1 \text {, the next LOL(4) values are the P } 2 \text { components of the elastic scattering cross } \\
\text { sections for transfer from group I to } \mathrm{J} \text { followed by the P } 3 \text { components from group I to group } \\
\mathrm{J} .\end{array}$ \\
\hline & $\begin{array}{l}\text { If } \operatorname{LOL} 5=1 \text {, the next } \operatorname{LOL}(5) \text { values are the } \mathrm{P} 0(\mathrm{n}, 3 \mathrm{n}) \text { cross sections for transfer from group I } \\
\text { to group } \mathrm{J} . \text { The list is ordered as above for the inelastic data. There is no } \mathrm{P} 1(\mathrm{n}, 3 \mathrm{n}) \text { matrix. }\end{array}$ \\
\hline & $\begin{array}{l}\text { If LOL6 }=1 \text {, the next } \operatorname{LOL}(6) \text { values are the } \mathrm{P} 0(\mathrm{n}, 4 \mathrm{n}) \text { cross sections for transfer from group I } \\
\text { to group J. The list is ordered as above for the inelastic data. There is no P1 }(n, 4 n) \text { matrix. }\end{array}$ \\
\hline
\end{tabular}




\subsection{Thermal $S(\alpha, \beta)$ and Free Scattering Cross Section Internal Record}

itfile $=449+$ is

1 = group scattered from

$\mathrm{jj}=$ group scattered to

$\mathrm{ljj}=(1-1)^{*} \operatorname{maxg}+\mathrm{jj}$

write (itfile) 1,jj,therm0(ljj),therm1(lij),therm2(lij),therm3(lij)

where

$1 \leq$ is $\leq$ noi $\quad$ (noi $=$ number of materials in the problem),

$65 \leq 1 \leq 167$ (fine groups in the thermal energy range),

$65 \leq \mathrm{jj} \leq 167 \quad$ (fine groups in the thermal energy range), 


\section{PROGRAMMER'S GUIDE}

COMBINE7.1 is written in FORTRAN 90 and is currently compiled using the Intel Visual Fortran compiler or the Lahey/Fujitsu (LF95) Fortran compiler on the Windows 32 platform. The Intel Visual Fortran compiler provides the faster run time. The source code is heavily commented to explain subroutine functions, data structures, etc. This section provides a short description of each subroutine and shows the overlay structure.

\subsection{Flow Diagram}
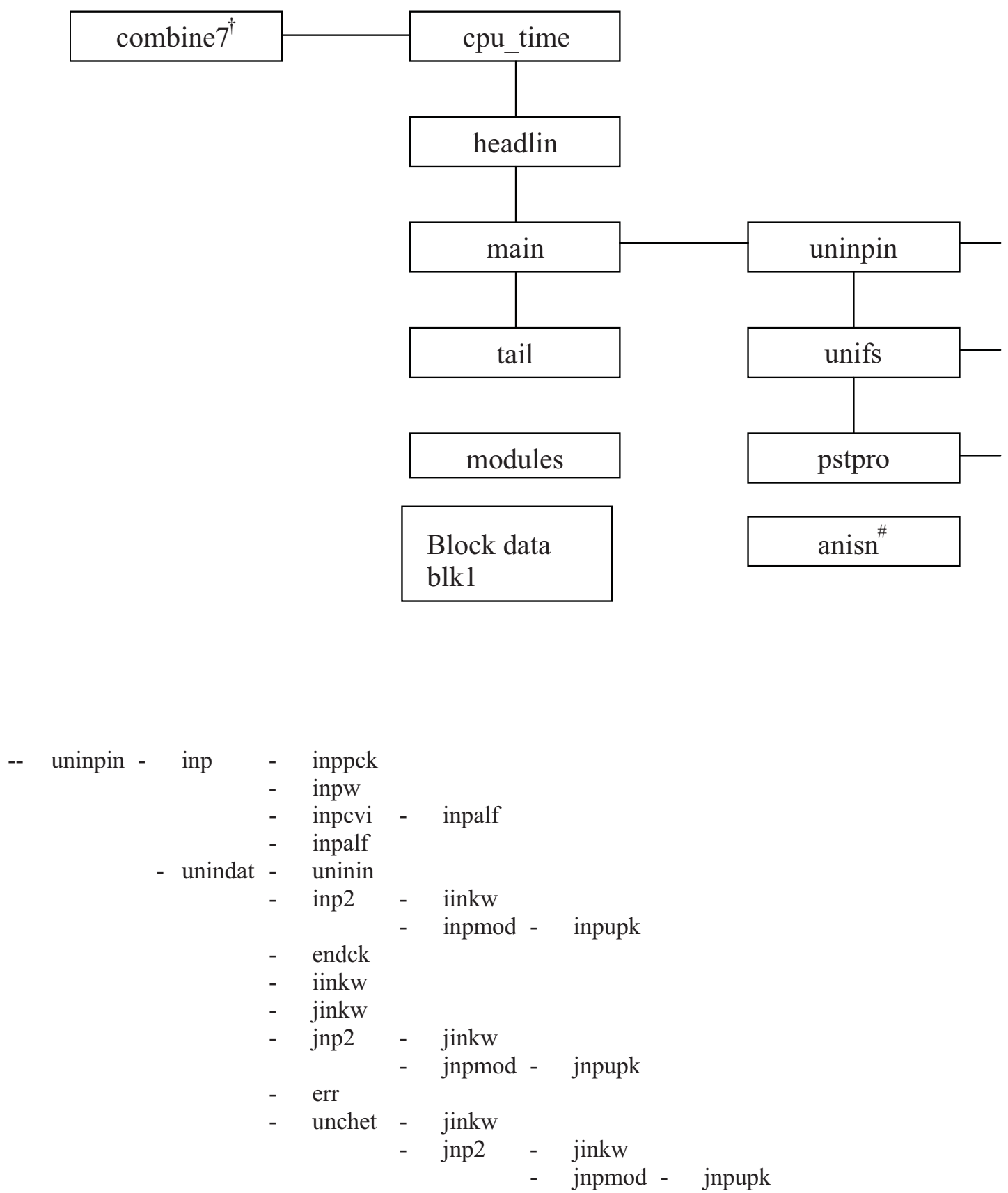


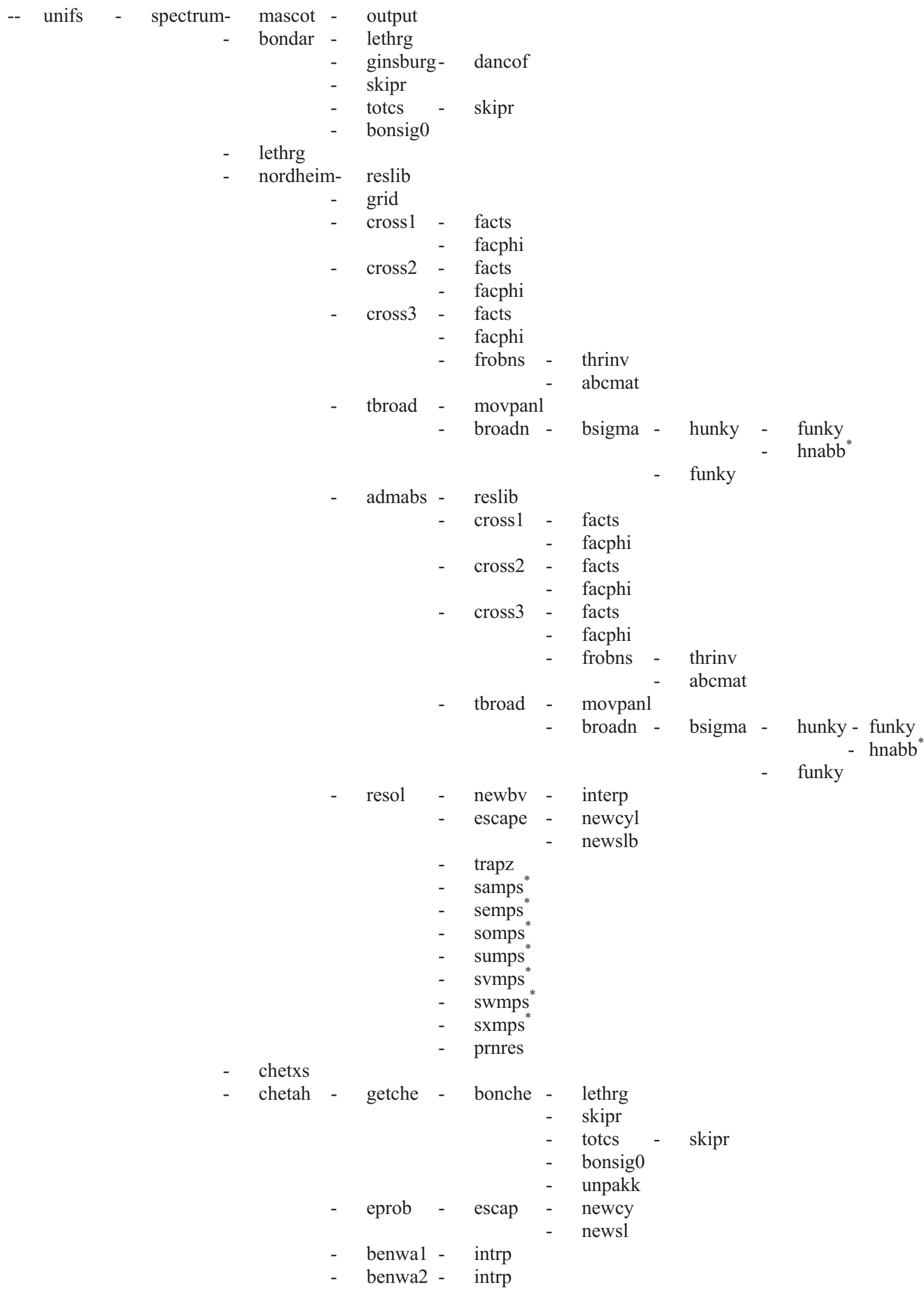




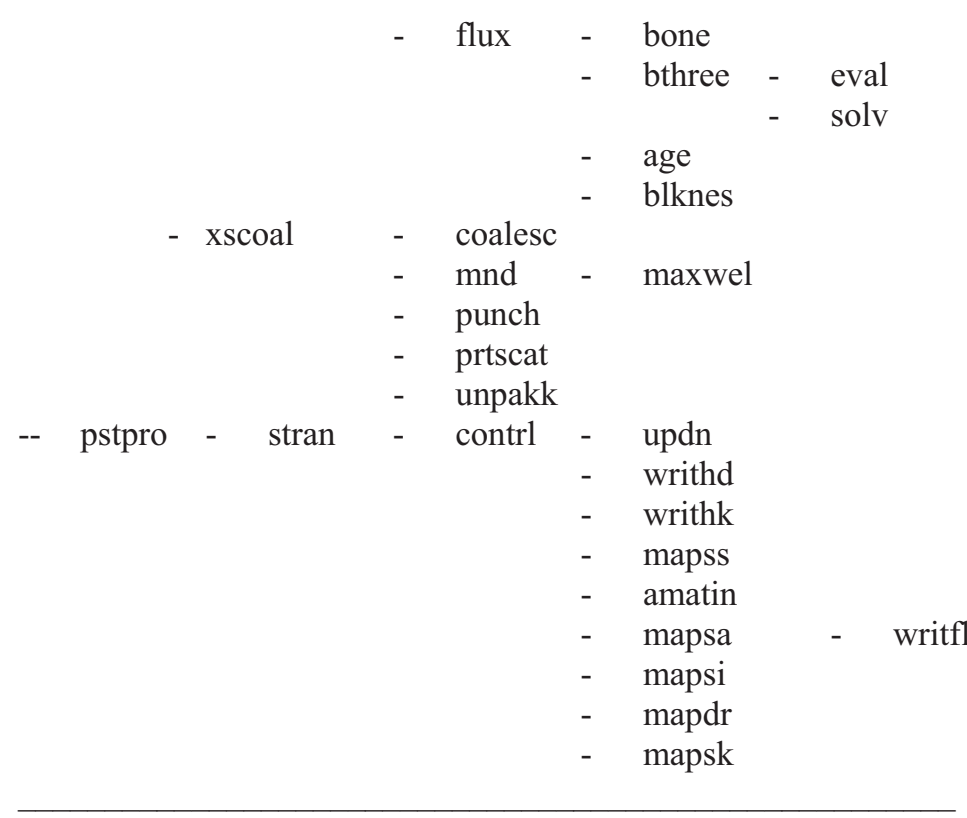

Note 1: † indicates "program",

* "function",

otherwise, "subroutine".

\# see ANISN manual.

Note 2: SR.QUIT is used at several places to close temporary files and terminate execution.

An intrinsic subroutine, "exit" is used to terminate execution, close all files, and return control to the operating system. 


\subsection{Summary of Contents of Subroutines}

\subsubsection{Program COMBINE7.1 and Supporting Subroutines}

Overall control of COMBINE7.1 is maintained in PROGRAM COMBINE7.1. It controls the flow of the overall program. COMBINE7.1 calls SR.MAIN to read and digest all of the input data, perform spectrum calculation, and process the cross-section output data into the user-specified final format. It also calls the date and time subroutines to produce the banner information printed at the beginning and end of the printed output.

\section{SR. ABCMAT}

Routine to do a matrix multiplication. Called from sr.frobns.

\section{SR. ADMABS}

Generates cross sections from the admixed absorber resonances.

\section{SR.AGE}

AGE calculates moments and Fermi age from the second moment when an age calculation is requested. These are Equations (137) through (143) in the text.

\section{SR.AMATIN}

Processes the neutral internal cross-section file prior to reformatting.

\section{SR.BENWA1}

BENWA1 calculates thermal disadvantage factors at each energy point for slab geometry. The slab geometry equations are Equations (172) to (176) in the text. The weights are then used in SR.CHETXS to calculate selfshielding factors for each material in the three region slab geometry cell described in the input data. BENWA1 is called from SR.CHETAH.

\section{SR.BENWA2}

BENWA2 calculates thermal disadvantage factors at each energy point for cylindrical geometry. The cylindrical geometry equations are Equations (166) to (175) in the text. The weights are then used in SR.CHETXS to calculate self-shielding factors for each material in the three region, cylindrical geometry cell described in the input data. BENWA2 is called from SR.CHETAH.

\section{BLOCK DATA BLK1}

Collision Probabilities from case and Placzek pin locations for dancoff calculation.

\section{SR.BLKNES}

BLKNES calculates thermal blackness coefficients for diffusion theory codes. BLKNES is called from SR.FLUX.

\section{SR.BONCHE}

Returns Bondarenko shielded cross sections. This routine is used when ABH calculation is requested.

\section{SR.BONDAR}

Bondarenko self-shields and transfers all requested library material cross sections to the file unit 419 . The file unit 419 is then library file with no additional material to be processed in SR. SPECTRUM.

\section{SR.BONE}

BONE solves the B-1 or P-1 groupwise fast spectrum equations by substitution. The solution proceeds from group 1 through group 167 with the thermal upscattering iterations. These are Equations (33) and (34) in the text. The 167 group isotropic neutron flux and current are returned. BONE is called from SR.FLUX.

\section{SR.BONSIG0}

Calculates the groupwise Bondarenko background cross sections for the Bondarenko self shielding. Includes the heterogeneous component.

\section{SR. BROADN}

Doppler broaden the resolved resonance cross sections.

\section{SR.BSIGMA}

A part of Doppler broadening algorithm. Called from SR.BROADN.

\section{SR.BTHREE}

BTHREE solves the B-3 groupwise fast spectrum equations by the Gauss-Seidel iteration. These are Equations 
(26) through (30) in the text. The groupwise solution proceeds from group 1 through group 167 with the thrmal upscattering iteration. Four Legendre moments for the B-3 fluxes are returned for each microgroup. BTHREE is called from SR.FLUX.

\section{SR.CHETAH}

Controls the calculation of thermal disadvantage factors when ABH calculation is requested. The actual disadvantage factors are calculated in SR.BENWA1 for the slab geometry and SR.BENWA2 for the cylindrical geometry. CHETAH is called from SR.SPECTRUM.

\section{SR.CHETAH}

CHETAH controls the calculation of the thermal disadvantage factors when an ABH calculation is requested in the input. The actual disadvantage factors are calculated in SR.BENWA1 for slab geometry and SR.BENWA2 for cylindrical geometry.

\section{SR.CHETXS}

Corrects cross section in the thermal energy range by ABH's thermal disadvantage factors and substitute microscopic cross sections. Apply only to absorption, fission, and elastic cross section since inelastic, (n,xn) occur at epithermal energy range.

\section{SR.COALSEC}

COALSEC calculates the broad group fluxes, currents, and cross sections from the fast spectrum flux/current weighted values. It is called to calculate both macroscopic and microscopic values based on Equations (236) through (266) in the text. COALSEC is called from SR.XSCOAL.

\section{SR.CONTRL}

Controller for final formatting of the ASCII cross-section output file.

\section{SR.CROSS1}

CROSS1 calculates the single level Breit-Wigner resolved resonance cross sections on energy mesh generated in SR.GRID. Doppler-broadening occurs in SR.TBORAD.

\section{SR.CROSS2}

CROSS2 calculates the multi level Breit-Wigner resolved resonance cross sections on energy mesh generated in SR.GRID. Doppler-broadening occurs in SR.TBORAD.

\section{SR.CROSS3}

CROSS3 calculates the Reich-Moore resolved resonance cross sections on energy mesh generated in SR.GRID. Doppler-broadening occurs in SR.TBORAD.

\section{SR.DANCOF}

DANCOF calculates the Dancoff-Ginsburg factor $\mathrm{C}$ for a set of square lattice or hexagonal lattice pins in regular arrays depending upon the type of lattice requested in the input. The factor $\mathrm{C}(\mathrm{I})$ for the I resonance material is returned for each calculation. The calculation is for Equations (86) through (88) in the text. DANCOFF is called from SR.GINSBURG.

\section{SR.ENDCK}

ENDCK prints an error message when errors are detected on an input card.

\section{SR.EPROB}

Calls SR.ESCAP to calculate the escape probability, $\mathrm{P}_{0}$, for the cell thermal disadvantage factor calculation. The same capture probability tables are used that were constructed for the resolved resonance calculation in the fast spectrum code. EPROB is called from SR.CHETAH.

\section{SR.ERR}

ERR prints an error message indicating which items on a given input card are in error.

\section{SR.ESCAP}

Copy of SR.ESCAPE. Computes the escape probability, $\mathrm{P}_{0}$, to be used in the ABH solution. Used for overlay efficiency. Called from SR.EPROB.

\section{SR.ESCAPE}

ESCAPE calculates the heterogeneous lump escape probability, $\mathrm{P}_{0}$, used in Equation (40) for the solution of the resolved resonance collision density. ESCAPE calls SR.NEWCYL or SR.NEWSLB for table lookup values of cylindrical or slab geometries respectively. ESCAPE returns $\mathrm{P}_{0}$ the escape probability and is called from SR.RESOL. 


\section{SR.EVAL}

EVAL evaluates the B-3 coefficients $\mathrm{A}_{\mathrm{ij}}$ in Equations (24). EVAL is called from SR.BTHREE.

\section{SR.FACPHI}

Calculate phase shift for the resonance calculation. Called from SR.CROSS1, CROSS2, and CROSS3.

\section{SR.FACTS}

Calculates penetration and shift factors for the resonance calculation. Called from SR.CROSS1, CROSS2, and CROSS3.

SR.FLUX

Calls SR.BONE, BTHREE, AGE, and BLKNES for the B-1 spectrum, B-3 spectrum, age, and blakness calculation, respectively.

\section{SR.FROBNS}

Inverts a complex matrix. Called from SR.CROSS3.

\section{SR.FUNKY}

Designed to define functions that appear in Doppler broadening calculations.

\section{SR.GETCHE}

GETCHE reads thermal cross-section data for each material in the three regions, cell disadvantage factor calculation. It is called by CHETAH once for each region in the cell. Macroscopic cross sections are accumulated in each region for use in the disadvantage factor calculation.

\section{SR.GINSBURG}

A drive subroutine for Dancoff-Ginsburg factor calculaton.

\section{SR.GRID}

GRID calculates a discrete lethargy mesh in energy space which is used in SR.CROSS1, CROSS2, and CROSS3 to calculate the resonance cross sections used in solving Equation (40) for the collision density and Equation (56) for the resonance integrals in the resolved resonance range. The lethargy mesh is generated independently for each resonance from midpoint to midpoint between resonances. The mesh interval is directly proportional to the resonance width and inversely proportional to the resonance energy. A maximum of 900 uniform mesh intervals are permitted for each resolved resonance. However, the mesh spacing from resonance to adjacent resonance varies with gamma, the width of each resonance. The mesh spacing may be controlled by the input parameters FMULT and XLMT defined in the input instructions. The default values, however, produce optimum results. The mesh is therefore non-uniform across the series of resonance levels upstream from any given mesh point in order to calculate scatter-in sources when solving Equation (40). GRID is called from SR.NORDHEIM.

\section{SR.HEADLIN}

Prints banner page. Calls date and time.

\section{FUNCTION.HNABB}

Calculates $h n(a, b)$ for $b>=a$ by a direct Taylor series expansion of the defining integral. Used in Doppler broadening calculation.

\section{SR.HUNKY}

This routine is designed to define functions that appear in Doppler broadening calculations.

\section{SR.INP}

Driver for the free format input package. Sets up an array of input data and a table of pointers to the data to allow free field input processing. Supervises subroutines INPALF, INPCVI, INPPCK, INPW. See the extensive comments in these subroutines for details.

\section{SR.INP2}

Routine to transfer data from input card buffer to designated storage area in core. Supervises subroutines IINKW, INPMOD/INPUPK. See the extensive comments in these subroutines for details.

\section{SR.INTERP}

INTERP is called from SR.NEWBV which is called by SR.RESOL for the resolved resonance numerical solution. NEWBV is called by RESOL to set new back values for the scatter-in source on the right-hand side of Equation (40). INTERP is called by NEWBV to interpolate from old back values to the new back value mesh.

\section{SR.INTRP}

INTRP linearly interpolates the dependent variable $X Y$ from the input table $X, Y$, from the argument $X X$. INTRP is called from SR.BENWA1 and SR.BENWA2. 


\section{SR.JNP2}

Copy of SR.INP2. Supervises subroutines JINKW, JNPMOD/JNPUPK. Used for overlay efficiency.

\section{SR.LETHRG}

LETHRG calculates broad group energy boundaries and lethargy widths from the input cut point. LETHRG is called from SR.BONDAR.

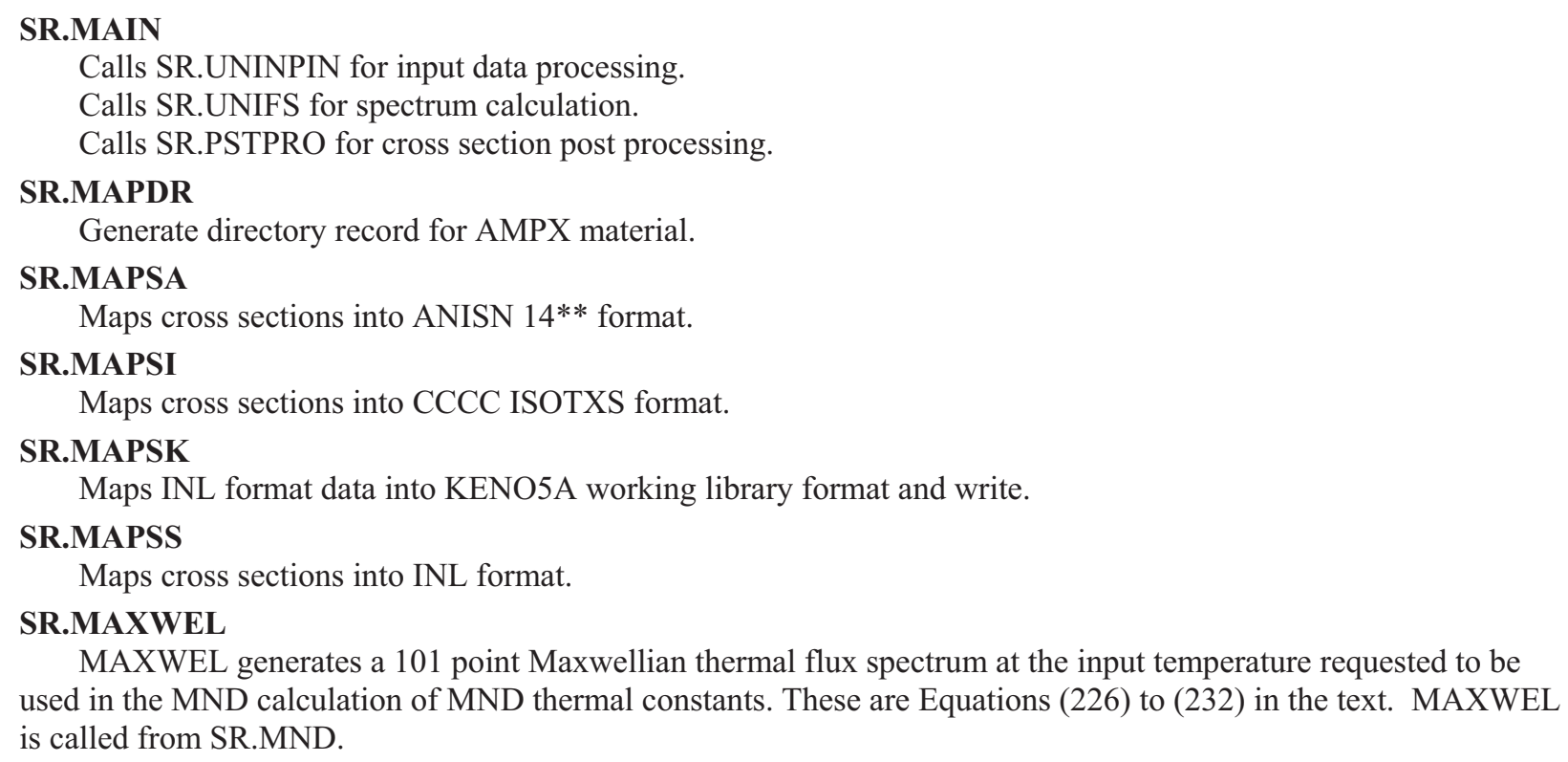

\section{SR.MAPSS}

Maps cross sections into INL format.

\section{SR.MAXWEL}

MAXWEL generates a 101 point Maxwellian thermal flux spectrum at the input temperature requested to be used in the MND calculation of MND thermal constants. These are Equations (226) to (232) in the text. MAXWEL is called from SR.MND.

\section{SR.MND}

MND calculates Mixed Number Density constants when requested in the input. These are given in Equations (226) to (232) in the text. MND constants may only be calculated for a single thermal energy group. The MND constants are then printed for scrutiny by the user. MND is called from SR.'s MICRO and INMN3.

\section{SR.MOVPANL}

Move panel for Doppler broadening. Called from SR.TBROAD.

\section{SR.NEWBV}

NEWBV is called by SR.RESOL to calculate a set of new back values for the scatter-in source on the righthand side of Equation (40). New back values are required because a new uniform mesh is required for Simpson's rule integration of the right-hand side of Equation (40) for the scatter-in source. SR.INTERP is called by NEWBV to interpolate to the new uniform mesh from old back values on the old non-uniform mesh generated for the crosssection calculation in SR.CROSS.

\section{SR.NEWCY}

Copy of SR.NEWCYL. Used for overlay efficiency.

\section{SR.NEWCYL}

NEWCYL is called by SR.ESCAPE to do the tabular interpolation for the capture probability $\mathrm{P}_{\mathrm{c}}$ for cylindrical pins. The escape probability $\mathrm{P}_{0}=1-\mathrm{P}_{\mathrm{c}}$.

\section{SR.NEWSL}

Copy of SR.NEWSLB. Used for overlay efficiency.

\section{SR.NEWSLB}

NEWSLB is called by SR.ESCAPE to do the tabular interpolation for the capture probability $\mathrm{P}_{\mathrm{c}}$ for slab geometry. The escape probability $\mathrm{P}_{0}=1-\mathrm{P}_{\mathrm{c}}$.

\section{SR.NORDHEIM}

Drive routine for Nordeim integral treatment for resonance integrals.

\section{SR.OUTPUT}

Writes out most of the data input. 


\section{SR.PRNRES}

PRNRES prints the results of the resolved resonance calculations by fine group. PRNRES is called from SR.NORDHEIM.

\section{SR.PRTSCAT}

PRTSCAT prints out the broad group scattering matrices for both macroscopic and microscopic cross-section data. PRTSCAT is called from SR.XSCOAL.

\section{SR.PSTPRO}

Controlled for ASCII flux and cross-section output file post processing.

\section{SR.PUNCH}

PUNCH formats and writes the device-specified broad group output data. PUNCH is called from SR.XSCOAL.

\section{SR.QUIT}

Exit subroutine. Closes files and terminates program.

\section{SR.MASCOT}

Initializes macroscopic scattering cross sections and calls SR.OUTPUT. Called from SR.SPECTRUM.

\section{SR.RESLIB}

Read resolve resonance parameters from the "reres.lib" file. Reverse the order from high to low energy.

\section{SR.RESOL}

RESOL performs the calculation of the resolved resonance integrals by means of the Nordheim numerical solution. It calls SR.CROSS to generate the Doppler-broadened cross sections from the resolved resonance parameters. It solves Equation (4) at each fine lethargy mesh point for the collision density. It constructs resonance integrals from Equation (56). Shielding of the elastic scattering matrices is performed using Equations (76) through (86) in the text. SR.PRNRES is then called to print the results of the resonance calculations at the completion of the unresolved resonance calculation. RESOL is called from SR.NORDHEIM.

\section{FUNCTION SAMPS}

SAMPS integrates the scatter-in source term on the right-hand side of Equation (40) for the principal resonance absorber by means of Simpson's rule. The integration is over the back-value mesh generated in SR.NEWBV. SAMPS is called from SR.RESOL.

\section{FUNCTIOM SEMPS}

SEMPS integrates the scatter-in source term on the right-hand side of Equation (40) for the first input moderator by means of Simpson's rule. The integration is over the back-value mesh generated in SR.NEWBV. SEMPS is called from SR.RESOL.

\section{SR.SKIPR}

Skip forward or backward on a coded or blocked binary tape. Works on the binary "matxs.lib."

\section{SR.SOLV}

SOLV solves the B-3 Equation (26) by means of block Gauss elimination one group a time from high to low in energy. SOLV is called from SR.BTHREE.

\section{FUNCTIOM SOMPS}

SEMPS integrates the scatter-in source term on the right-hand side of Equation (40) for the second input moderator by means of Simpson's rule. The integration is over the back-value mesh generated in SR.NEWBV. SEMPS is called from SR.RESOL.

\section{SR.SPECTRUM}

Drives spectrum calculation by first generating the required fine group cross sections and then calculating spectrum.

\section{SR.STRAN}

Driver for reformatting the ASCII cross-section output file from internal format to the user-requested final format. Sets up storage, etc.

\section{FUNCTIOM SUMPS}

SEMPS integrates the scatter-in source term on the right-hand side of Equation (40) for the third input moderator by means of Simpson's rule. The integration is over the back-value mesh generated in SR.NEWBV. SEMPS is called from SR.RESOL.

\section{FUNCTIOM SVMPS}

SEMPS integrates the scatter-in source term on the right-hand side of Equation (40) for the first input admixed 
resonance absorber by means of Simpson's rule. The integration is over the back-value mesh generated in SR.NEWBV. SEMPS is called from SR.RESOL.

\section{FUNCTIOM SWMPS}

SEMPS integrates the scatter-in source term on the right-hand side of Equation (40) for the second input admixed resoanance absorber by means of Simpson's rule. The integration is over the back-value mesh generated in SR.NEWBV. SEMPS is called from SR.RESOL.

\section{FUNCTIOM SXMPS}

SEMPS integrates the scatter-in source term on the right-hand side of Equation (40) for the third input admixed resonance absorber by means of Simpson's rule. The integration is over the back-value mesh generated in SR.NEWBV. SEMPS is called from SR.RESOL.

SR.TAIL

Prints final banner. Print date, time, and elapsed run time.

\section{SR.TBROAD}

Manages the paging of cross section data for Doppler broadening.

\section{SR.THRINV}

Inverts symmetric matrix. Called from SR.FROBNS.

\section{SR.TOTCS}

Read total cross section from vector blocks in "matxs.lib" for all sibmaterials and save on direct access file, 'is' unit.

\section{SR.TRAPZ}

TRAPZ performs a trapezoidal integration of seven dependent variables from SR.RESOL over uniform mesh for one energy group in the resolved resonance range. The seven integrals are returned. TRAPZ is called from SR.RESOL.

\section{SR.UNCHET}

UNCHET reads and prints the three region ABH disadvantage factor input data on the 2000000 series cards in the input description. UNCHET is called from SR.UNINDAT only when an ABH disadvantage factor calculation is requested.

\section{SR.UNIFS}

Calls SR.SPECTRUM, which drives the spectrum calculation. Also calls SR.XSCOAL, which drives the cross section coalescing.

\section{SR.UNINDAT}

UNINDAT reads the input integer data cards in the 1000000 series and maps the input data onto modules. The data are then checked for range errors and any errors are printed. UNINDAT is called from SR.UNINPIN.

\section{SR.UNININ}

UNININ initializes the input integer data arrays before the entry of change case data. This preserves unchanged values from the previous problem. UNININ is called from SR.UNINDAT.

\section{SR.UNINPIN}

UNINPIN controls the reading of all input data on 1000000 series cards using INP subroutines. SR.UNINDAT is called to map input data which is read onto modules. UNINPIN is called form PROGRAM COMBINE7.

\section{SR.UNPAKK}

Takes the cross section data present in the ADUM array read from unit 404(????) and packs them into the local variables. UNPAKK is called from SR.GETCHE and SR.XSCOAL.

\section{SR.UPDN}

Find the largest upscatter distance, largest downscatter distance, count total number of materials in the file, and manufacture an ISOTXS 6-character name for each material.

\section{SR.WRITFL}

Utility for writing ANISN, free form, ASCII input using repeats and fills as appropriate.

\section{SR.WRITHD}

Writes header information if ISOTXS cross-section output is specified.

\section{SR.WRITHK}

Writes header for KENO5 fromat. 


\section{SR.XSCOAL}

Drives the coalescing option. 


\section{REFERENCES}

1. National Nuclear Data Center, Evaluated Nuclear Data File (ENDF/B-VII.0), Brookhaven National Laboratory, December 15, 2006.

2. R. A. Grimesey, David W. Nigg, Richard L. Curtis, COMBINE/PC-A Portable ENDF/B Version 5 Neutron Spectrum and Cross-Section Generation Program, EGG-2589 Rev.1, February 1991.

3. R. L. Curtis et al., PHROG -A FORTRAN-IV Program to Generate Fast Neutron Spectra and Average Multigroup Constants, Idaho Nuclear Corp., IN-1435, April 1971.

4. R. L. Curtis and R. A. Grimesey, INCITE: A FORTRAN-IV Program to Generate Thermal Neutron Spectra and Multigroup Constants Using Arbitrary Scattering Kernels, Idaho Nuclear Corp., IN-1062, 1967.

5. National Nuclear Data Center, Evaluated Nuclear Data File (ENDF/B-V), Brookhaven National Laboratory, 1979. R. Kinsey, Data Formats and Procedures for the Evalusted Nuclear Data File ENDF, BNL-NCS-50496, 1979.

6. National Nuclear Data Center, Evaluated Nuclear Data File (ENDF/B-VI), Brookhaven National Laboratory, 1990. V. McLane, Editor, ENDF-102 DATA FORMATS AND PROCEDURES FOR THE EVALUATED NUCLEAR DATA FILE ENDF-6, BNL-NCS-44954-01/04-REV, July 1990.

7. R. E. MacFarlane and D. W. Muir, The NJOY Nuclear Data Processing System Version 91, Loas Alamos National Laboratory, LA-12740-M, October, 1994.

8. I. I. Bondarenko, Ed., Group Constants for Nuclear Reactor Calculations, Consultants Bureau, New York, 1964.

9. G. I. Bell and S. Glasstone, Nuclear Reactor Theory, Van Nostrand Reinhold, New York, 1970.

10. R. E. MacFarlane, TRANSX 2: A code for Interfacing MATXS Cross-Section Libraries to Nuclear Transport Codes, LA-12312-MS, RSICC PSR-317, Los Alamos National Laboratory, July 1992.

11. L. W. Nordheim, A Program of Research and Calculation of Resonance Absorption, GA-2527, 1961.

12. Internal Technical Report, ENDF/B Version 6 Nuclear Data Processing at INEL, W. Y. Yoon, R. A. Grimesey, and C. A. Wemple, NRRT-N-91-034, 1991. Internal Letter Report, W. Y. Yoon to D. W. Nigg, COMBINE-6 Cycle, WYY-01-94, E\&G Idaho, 1994.

13. G. D. Joanou and J. D. Dudek, GAM-I: A Consistent P-1 Multigroup code for the Calculation of Fast Neutron Spectra and Multigroup Constants, GA-1850, June 1961.

14. ANISN/PC Manual, D. Kent Parsons, EGG-2500, Idaho National Engineering Laboratory, December, 1988.

15. NJOY99.0 Code System for Producing Pointwise and Multigroup Neutron and Photon Cross Sections from ENDF/B data, RSICC PSR-480, March 2000. Processing ENDF/VII.0 with NJOY99.161 (the latest being NJOY99.259), National Nuclear Data Center, December 15, 2006.

16. D. Selengut, Critical Mass Calculations for Bare Hydrogen Moderated Reactors by Means of Transport Theroy, APEX-121, September 1952.

17. R. E. MacFarlene, R. B. Kidman and R. J. LaBauve, "The Background Cross Section Method as A General Tool for Reactor Anlaysis," CONF-78040, Proc. ANS Topical Meeting, Gatlinburg, Tennessee, April 1978, E. G. Silver, Ed. 1978.

18. M. G. Stamtelatos, R. J. LaBauve (LASL), "Space Shielding Cross Sections for Pebble-Bed High-Temperature Reactors," American Nuclear Society Transactions, 1972, pp. 600-601. 
19. M. Weinberg and E. P. Wigner, The Physical Theory of Neutron chain Reactors, Chicago: The University of Chicago Press, 1958.

20. K. M. Case, F. de Hoffman, and G. Placzek, Introduction to the theory of Neutron Diffusion, Los Alamos, NM: Los Alamos Scientific Laboratory, 1953.

21. Sauer, "Thermal Utilization in the Square Cell," Journal of Nuclear Energy, 18, 1964, p. 425.

22. G. D. Joanou and J. S. Dudek, GAM-II A B3 Code for the Calculation of Fast Neutron Spectra and Associated Multigroup Constants, GA-4265, September 1963L. Dresner, Resonance Absorption in Nuclear Reactors, New York: Pergamon Press, 1960.

23. M. G. Stamatelatos, "Rational Approximations for Cross-Section Space-Shielding in Doubly Heterogeneous Syatems," Nuclear Scoence and Engineering, 61, 4, 1976, pp. 543-549.

24. L. Dresner, Resonance Absorptionin Nuclear Reactors, New York; Pergamon Press, 1960.

25. D. E. Cullen, "Program SIGMA1 (version 77-1): Doppler Broaden Evaluated Cross Sections in the Evaluated Nucelar Data File/Version B (ENDF/B) Format," Lawrence Livermore National Laboratory report UCRL-50400, Vol. 17, Part B, 1977

26. N. M. Greene, L. M. Petrie, and R. M. Westfall, NITAWL-III: SCALE System Module for Performing Resonance Shielding and Working Library Production, pp. F2.3.2, NUREG/CR-0200, Rev 7, Volume II, Section F2, 2004.

27. K. R. Knight, SUPERDAN: Computer Programs for Calculating the Dancoff Factor of Spheres, Cylinders, and Slabs, ORNL/NUREG/CSO/Tm-2, March, 1978.

28. G. I. Bell, "A simple Treatment for Effective Resonance Absorption Cross Sections in Dense Lattices," Nuclear Science and Engineering, 5, 2, 1959, pp.138, 139.

29. H. Gelling and A.Sauer, Programmbechribung zu Dancoff Jr., IBM-7040, KEA-114, April 1964.

30. Amouyal, P. Benoist and J. Horowitz, "New Method for Determining the Thermal Utilization Factor in a Unit Cell," Journal of Nuclear Energy, 6, 1957.

31. L. E. Strawbridge, Calculation of Lattice parameters and Criticality for Uniform Water Moderated Lattices, WCAP-3269-25, September 1963.

32. G. E. Putnam, MONA A Multigroup One-Dimensional Neutronics Analysis Code, Aerojet Nuclear Company, ANCR-1051, 1972.

33. Michel H. Theys, "Integral Transport theory of Thermal Utilization Factor in Infinite Slab Geometry," Nuclear Science and Engineering, 7, 1, 1960, pp. 58-63.

34. W. Maynard, Blackness Theory and Coefficients for Slab Geometry, WAPD-TN-168, May 1959.

35. F. Henry, A theoretical Method for Determining the Worth of Control Rods, WAPD-218, August 1959.

36. D. Voorhis et al., Experimental and Theoretical Study of Critical Slabs: Effect of Absorbing Membranes of Cadmium, Gold, and Boron, WAPD-170, April 1957.

37. Radkowsky (ed.), Naval Reactors Handbook, Volume I, Selected Basic Techniques, TID-7050, 1964.

38. J. B. Fussell ltr to H, L. McMurry, JBF-8-70, SCAMP $\mathrm{S}_{\mathrm{n}}$ Code Consolidation and Modification, EG\&G Idaho, August 7, 1970,

39. J. Lieberoth and A. Stojadinovic,'Neutron Streming in Pebble Beds," Nuclear Science and Engineering, 76, 1980, pp336-344. 


\section{APPENDIX A}

\section{INL CROSS-SECTION FORMAT DESCRIPTION}




\section{Appendix A \\ INL Cross-Section Format Description}

The internal INEL format is based on a word-addressable card image file structure. The following tables describe this structure. A nonzero value in column 1 of a data record for a material signifies the end of data for that material The scatter matrices do not have the $2 \mathrm{~L}+1$ term multiplied into them.

Table A-1. The library material title record summary.

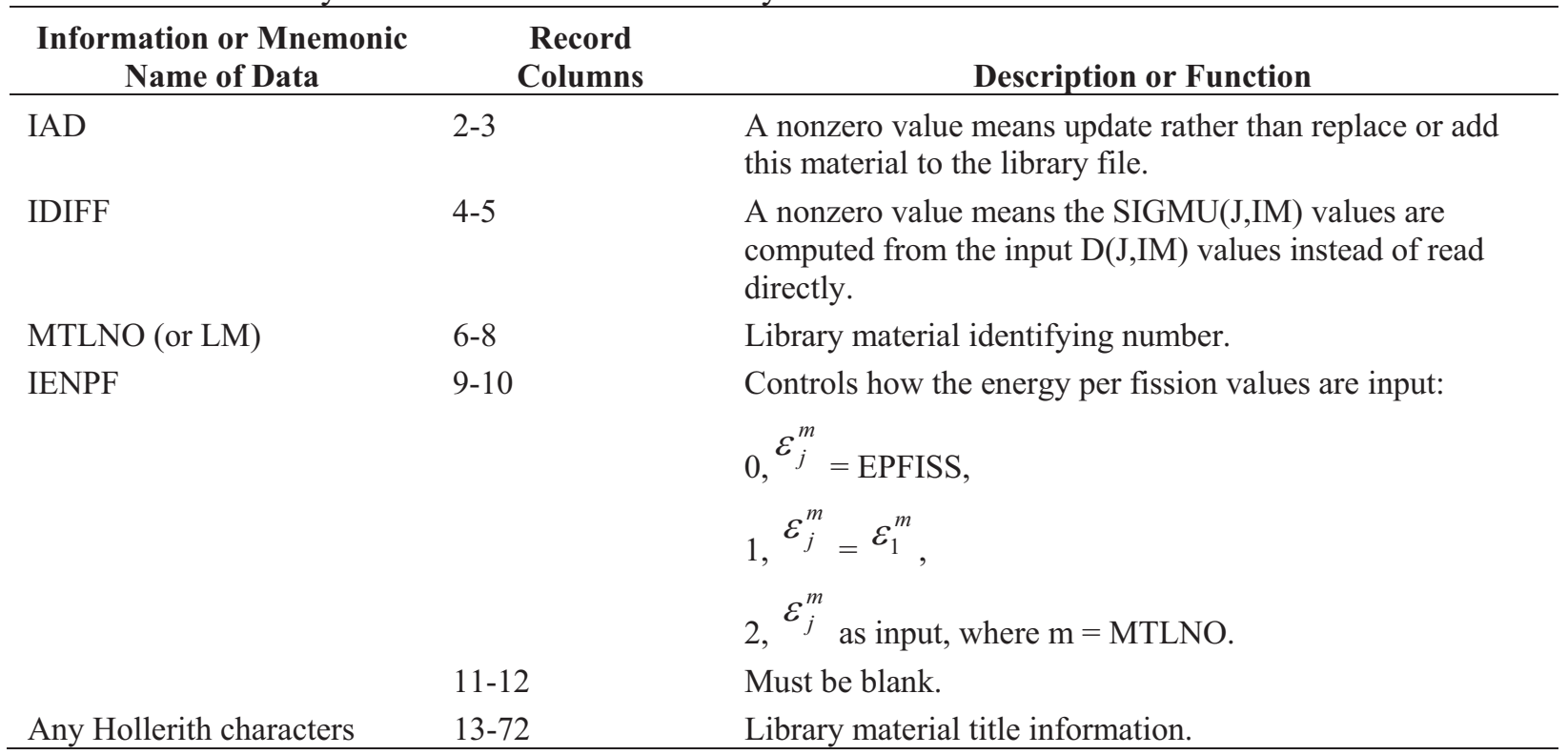

Card 2 for kinf and keff for the macroscopic data requested,

Energy per fission for the microscopic data requested. 
Table A-2. The library material floating point data summary.

\begin{tabular}{|c|c|c|c|c|c|}
\hline $\begin{array}{l}\text { Index } 1 \\
\text { Card } \\
\text { Cols. } \\
2-3 \\
\end{array}$ & $\begin{array}{c}\text { Index } 2 \\
\text { Card } \\
\text { Cols. } \\
4-5 \\
\end{array}$ & $\begin{array}{l}\text { Index } 3 \\
\text { Card } \\
\text { Cols. } \\
6-8 \\
\end{array}$ & $\begin{array}{l}\text { Index } 4 \\
\text { Card } \\
\text { Cols. } \\
9-10 \\
\end{array}$ & $\begin{array}{l}\text { Mnemonic } \\
\text { Name of } \\
\text { Data }\end{array}$ & $\begin{array}{l}\text { Description or Function of Data (index which increases as more } \\
\text { data words are added across a card is enclosed in parenthesis) }\end{array}$ \\
\hline 1 & LM & $\mathrm{J}$ & 1 & $\mathrm{DC}(\mathrm{J}, \mathrm{LM})$ & $\begin{array}{l}\text { Diffusion coefficient for group J of library material LM (when IDIFF = } \\
1 \text { on the title card). (J) }\end{array}$ \\
\hline 1 & LM & $\mathrm{J}$ & 2 & SIGA(J,LM) & Absorption cross section for group J of library material LM. (J) \\
\hline 1 & $\mathrm{LM}$ & $\mathrm{J}$ & 3 & SIGNU(J,LM) & $\begin{array}{l}\text { Neutron per fission times fission cross section for group } \mathrm{J} \text { of library } \\
\text { material } \operatorname{LM}\left(v \sum_{f}\right) \text {. }\end{array}$ \\
\hline 1 & LM & $\mathrm{J}$ & 4 & SIGFIS(J,LM) & Fission cross section for group J of library material LM. (J) \\
\hline 1 & $\mathrm{LM}$ & $\mathrm{J}$ & 5 & C4CHI(J;LM) & Fission source for group J of library material LM. $\quad(\mathrm{J})$ \\
\hline 3 & LM & $\mathrm{J}$ & $\mathrm{K}$ & TRMAT(J,K) & $\begin{array}{l}\text { General scattering transfer cross sections } \sum_{s 0} \text {, from group } \mathrm{J} \text { to group } \mathrm{K} \\
\text { for library material } \mathrm{m} \equiv \mathrm{LM} \text { ( } \mathrm{K} \text { may be less than, equal to, or greater } \\
\text { than } \mathrm{J}) .(\mathrm{K})\end{array}$ \\
\hline 4 & $\mathrm{LM}$ & $\mathrm{J}$ & 0 & SIGMU(J,LM) & $\begin{array}{l}\text { Transport cross section } \sigma_{u j}^{m} \text { for group } \mathrm{j}=\mathrm{J} \text { of library material } \mathrm{m} \equiv \mathrm{LM} \\
\text { (used in group } \mathrm{j}=\mathrm{J} \text { for mixing the macroscopic cross section, } \sum_{u j}^{m} \text {, } \\
\text { from which the macroscopic diffusion coefficient, } D_{j}^{m} \text {, for } \\
\text { macroscopic material } \mathrm{m} \text { is computed). This input data is used only if } \\
\text { IDIFF }=0 \text { or blank on the title card.(J) }\end{array}$ \\
\hline 6 & $\mathrm{LM}$ & $\mathrm{J}$ & $\mathrm{K}$ & TRMAT1(J,K) & Same as INDEX1 $=3$ for values of $\sum_{s 1}^{m}(\mathrm{~K})$ \\
\hline 7 & $\mathrm{LM}$ & $\mathrm{J}$ & $\mathrm{K}$ & TRMAT2(J,K) & Same as INDEX1 $=3$ for values of $\sum_{s 2}^{m}$ \\
\hline 8 & LM & $\mathrm{J}$ & $\mathrm{K}$ & TRMAT3(J,K) & Same as INDEX1 $=3$ for values of $\sum_{s 3}^{m} \quad(\mathrm{~K})$ \\
\hline
\end{tabular}




\section{APPENDIX B}

\section{ANISN CROSS-SECTION FORMAT DESCRIPTION}




\section{Appendix B \\ ANISN Cross-Section Format Description}

If the ANISN output format is selected, all cross sections are output in the standard ANISN card image $14^{* *}$ format (see Reference B-1 for details). The absorption, nu-sigma-fission, and total cross sections are always present regardless of the scattering order of the material or the current group number. For scattering orders beyond

$\mathrm{e}=0$, the absorption, nu-sigma-fission, and total cross sections are 0.0 .

The upscatter cross sections are present only when (HIS-IHT) is greater than 1. If they are present, there are NUS = (HIS - IHT - 1) locations present for every group and for every scattering order (material). This includes the physically nonexistent quantities such as $\sigma$ upscattering from group (IGM + NUS) to the lowest energy group IGM. In this way, the number of upscatter cross sections for each group in the cross-section table remains constant. The cross-section arrangement is summarized in Table B-1.

Table B-1. Cross-section arrangement.

\begin{tabular}{|c|c|c|}
\hline Cross Section & Group & Position Description \\
\hline$\sigma$ absorption & $\mathrm{g}$ & \\
\hline$v \sigma$ fission & $\mathrm{g}$ & \\
\hline$\sigma$ total or $\sigma$ transport & g & IHT \\
\hline$\sigma$ upscatter & $(g+N U S) \rightarrow g$ & \\
\hline$\bullet$ & $\bullet$ & \\
\hline • & $\bullet$ & \\
\hline$\bullet$ & $\bullet$ & \\
\hline$\sigma$ upscatter & $(g+1) \rightarrow g$ & IHS \\
\hline$\sigma$ self-scatter & $\mathrm{g} \rightarrow \mathrm{g}$ & \\
\hline$\sigma$ downscatter & $(g-1) \rightarrow g$ & \\
\hline • & $\bullet$ & \\
\hline$\bullet$ & $\bullet$ & \\
\hline - & $\bullet$ & \\
\hline$\sigma$ downscatter & $(g-N D S) \rightarrow g$ & IHM \\
\hline$\sigma$ upscatter (code calculated) & From $\mathrm{g}$ & $\mathrm{IHP}=\mathrm{IHM}+1$ \\
\hline
\end{tabular}

Finally, it should be noted that the higher-order scattering materials are output by COMBINE/PC without the $(2 \ell+1)$ term premultiplied into them.

\section{B. REFERENCE}

1. D. K. Parsons, ANISN/PC Manual, EGG-2500, December 1988. 


\section{APPENDIX C}

\section{CCCC INTERFACE FILE FORMATS AND CONVENTIONS}




\section{Appendix C \\ CCCC Interface File Formats and Conventions ${ }^{a}$ \\ ISOTXS-Nuclide (Isotope) Ordered, Multigroup Neutron Cross-Sections}

The changes in ISOTXS-IV relative to ISOTXS-III are in the main part, clarifications of ambiguities present in the latter. The changes reflected in Version IV are:

- If scattering bandwidth, $\operatorname{JBAND}(\mathrm{J}, \mathrm{N})$, is zero, no scatter data are present in block N. (see 4D record.)

- The position of self-(or within-group-) scatter must liewithin the scattering bandwidth unless the bandwidth is zero, i.e.,

I $<\operatorname{IJJ}(\mathrm{J}, \mathrm{N})<\operatorname{JBAND}(\mathrm{J}, \mathrm{N})$ if $\operatorname{JBAND}(\mathrm{J}, \mathrm{N}) \neq 0$.

- (See 4D record.)

- The Legendre expansion coefficient factor $(2 \rho+1)$ is not included in the P $\rho$ weighted transport and total cross sections. (See 5D record.)

- The $\mathrm{n}, 2 \mathrm{n}$ principal cross section (see $5 \mathrm{D}$ record) is clarified as the $\mathrm{n}, 2 \mathrm{n}$ reaction cross section while the $\mathrm{n}, 2 \mathrm{n}$ scatter matrix terms are clarified (see 4D record NOTE) as emission (productin) based, i.e.,

$\sigma_{n, 2 n(g)}=0.5 \sum_{g^{\prime}} \sigma_{n, 2 n}\left(g \rightarrow g^{\prime}\right)$

In addition, the following is included to clarify the meaning of the vectors IDSCT(N) and LORD(N) contained in the ISOTOPE CONTROL AND GROUP-INDEPENDENT DATA (4D) record and to remove an ambiguity in the subblocking of scattering data.

IDSCT(N) specifies the identity and ordering of scattering data blocks, and LORD(N) specifies the number of Legendre orders contained in each block. Each block of scattering may be subblocked into NSBLOCK records (subblocks) where NSBLOCK is found on the FILE CONTROL (ID) record. With the single exception below, sub-blocking shall not be used (NSBLOCK > 1) if the individual blocks defined by IDSCT and LORD contain more than one Legendre order of scattering. The single exception is that subblocking may be used with more than one Legendre order per record (subblock) only if each record (subblock) contains data for a single "scattered-into" group.

Finally, the following information is reproduced from the Version III specifications for the sake of completeness.

The $\mathrm{P}_{l}$ weighted transport and total cross sections can be defined ${ }^{\mathrm{b}}$ as

$$
\sigma_{n, 22,(g)}=0.5 \sum_{g^{\prime}} \sigma_{n, 2 n}\left(g \rightarrow g^{\prime}\right) \rho=1, \ldots, \mathrm{LTRN},
$$

and

a. Extracted from LA-6941-MS, Standard Interface Files and Procedures for Reactor Physics Codes Version IV by R. Douglas O'Dell.

b. Other definitions of the $\mathrm{P}_{l}$ weighted transport cross section are also possible. 
The P $\rho$ weighted scattering cross sections given in the scattering blocks are defined as

$$
\begin{aligned}
& \sigma_{t \rho}^{g} \equiv \frac{\int_{g} \sigma_{\rho}(E) d E}{\int_{g} \sigma_{\rho}(E) d E} \rho=0,1, \ldots, \text { LTOT-1 . } \\
& \sigma_{s \rho}\left(g \rightarrow g^{\prime}\right) \equiv \frac{\int_{g} \int_{g} \sigma_{s \rho}\left(E \rightarrow E^{\prime}\right) \sigma_{\rho}(E) d E^{\prime} d E}{\int_{g} \sigma_{\rho}(E) d E}
\end{aligned}
$$

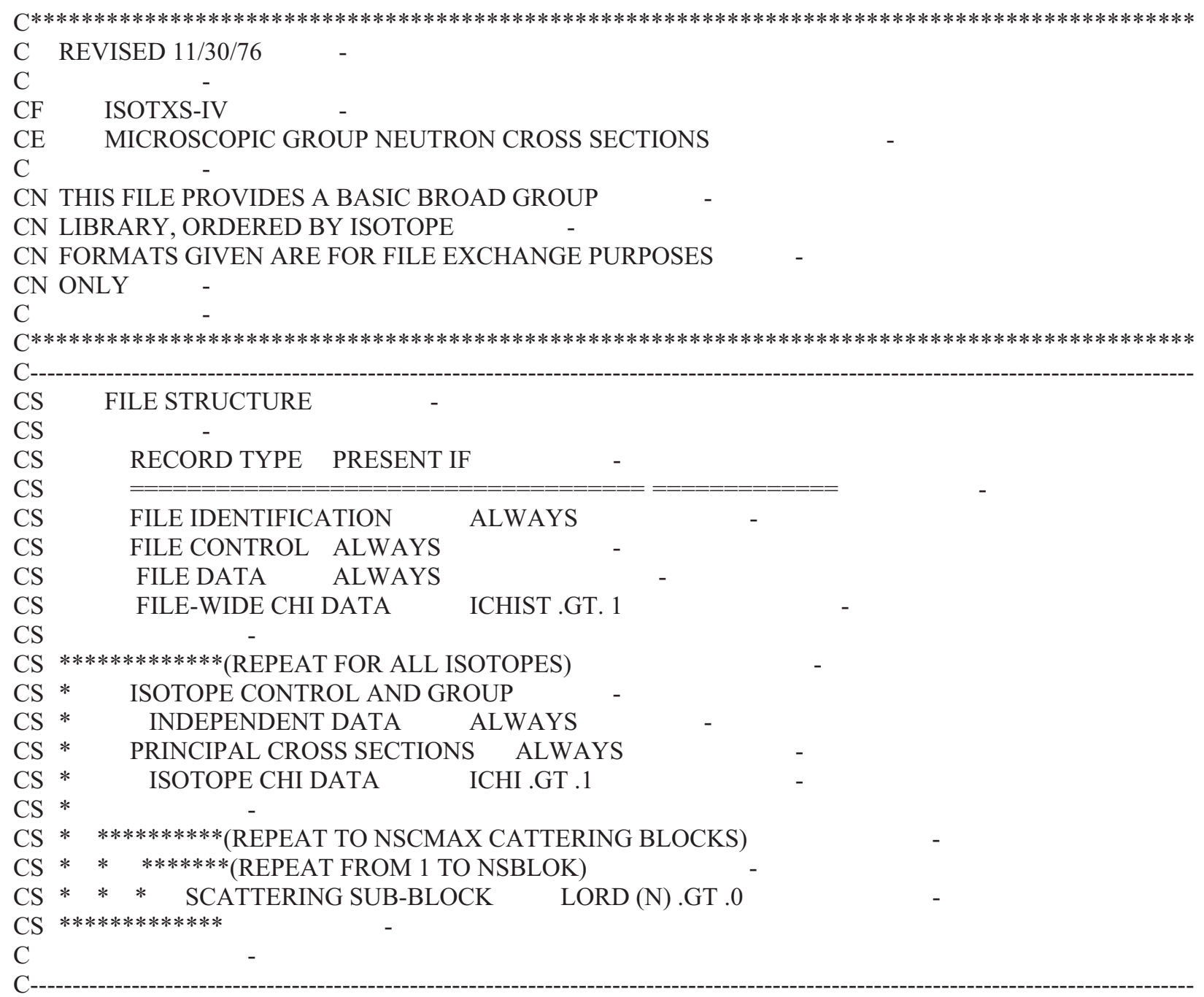




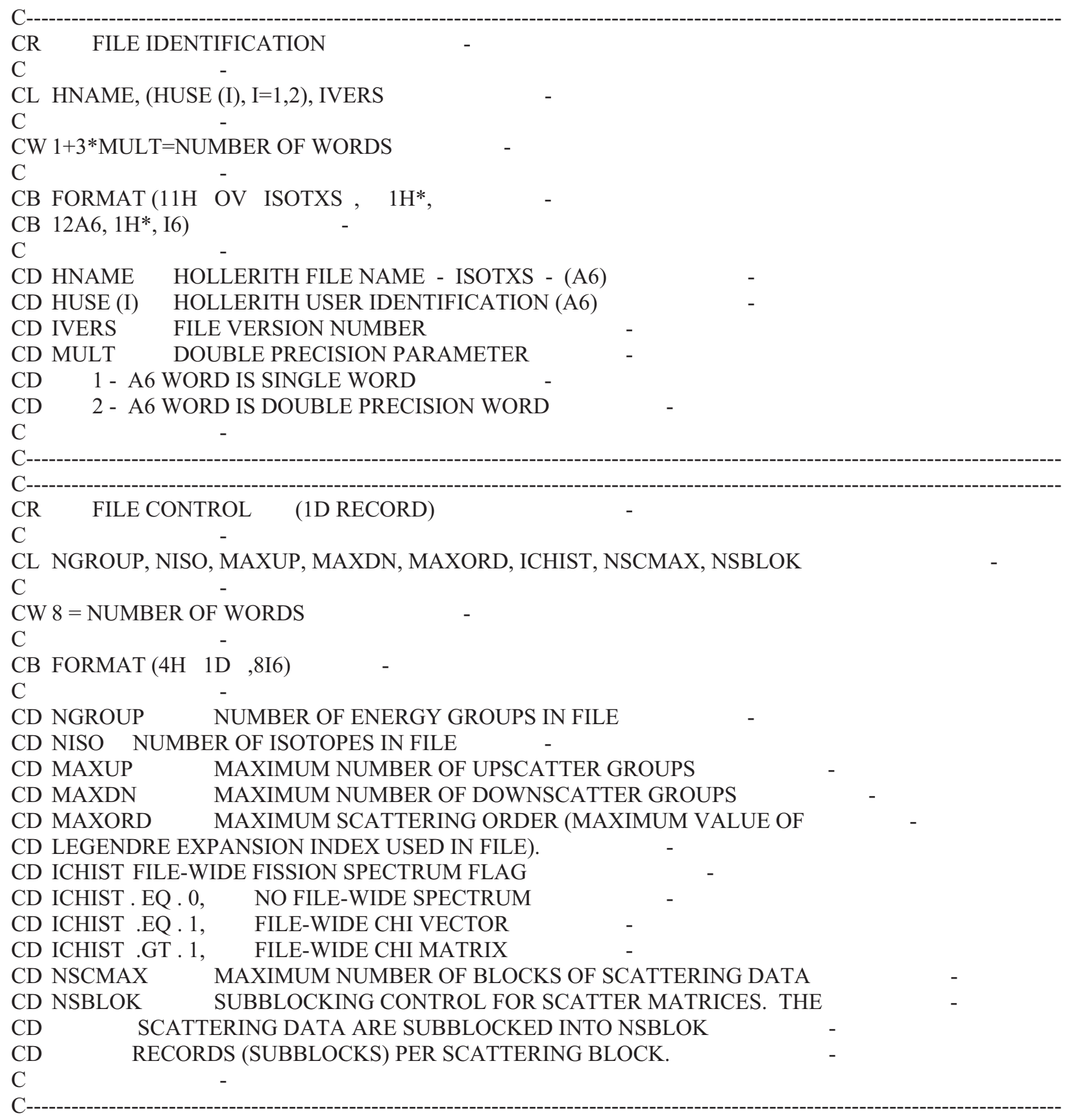




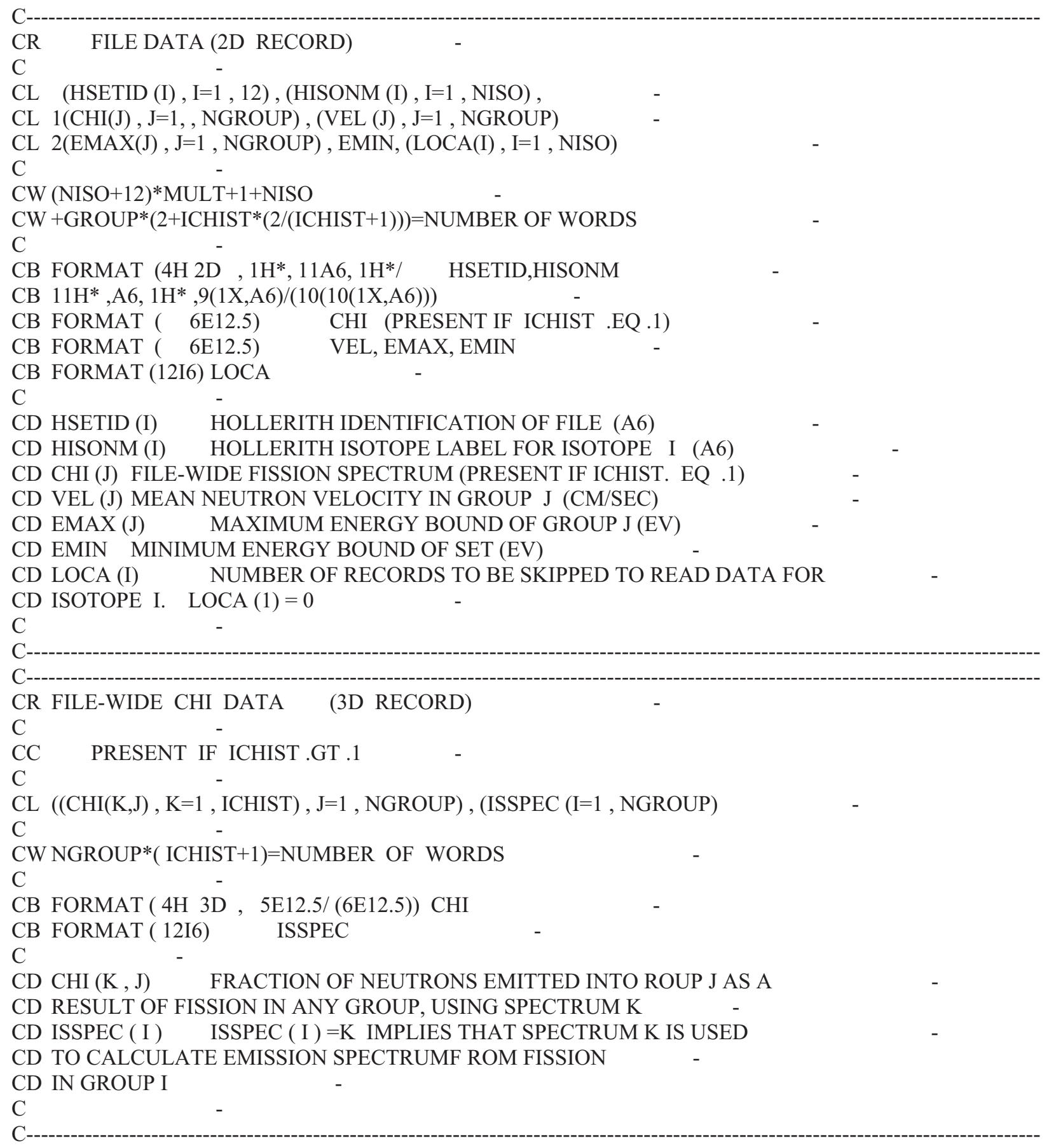




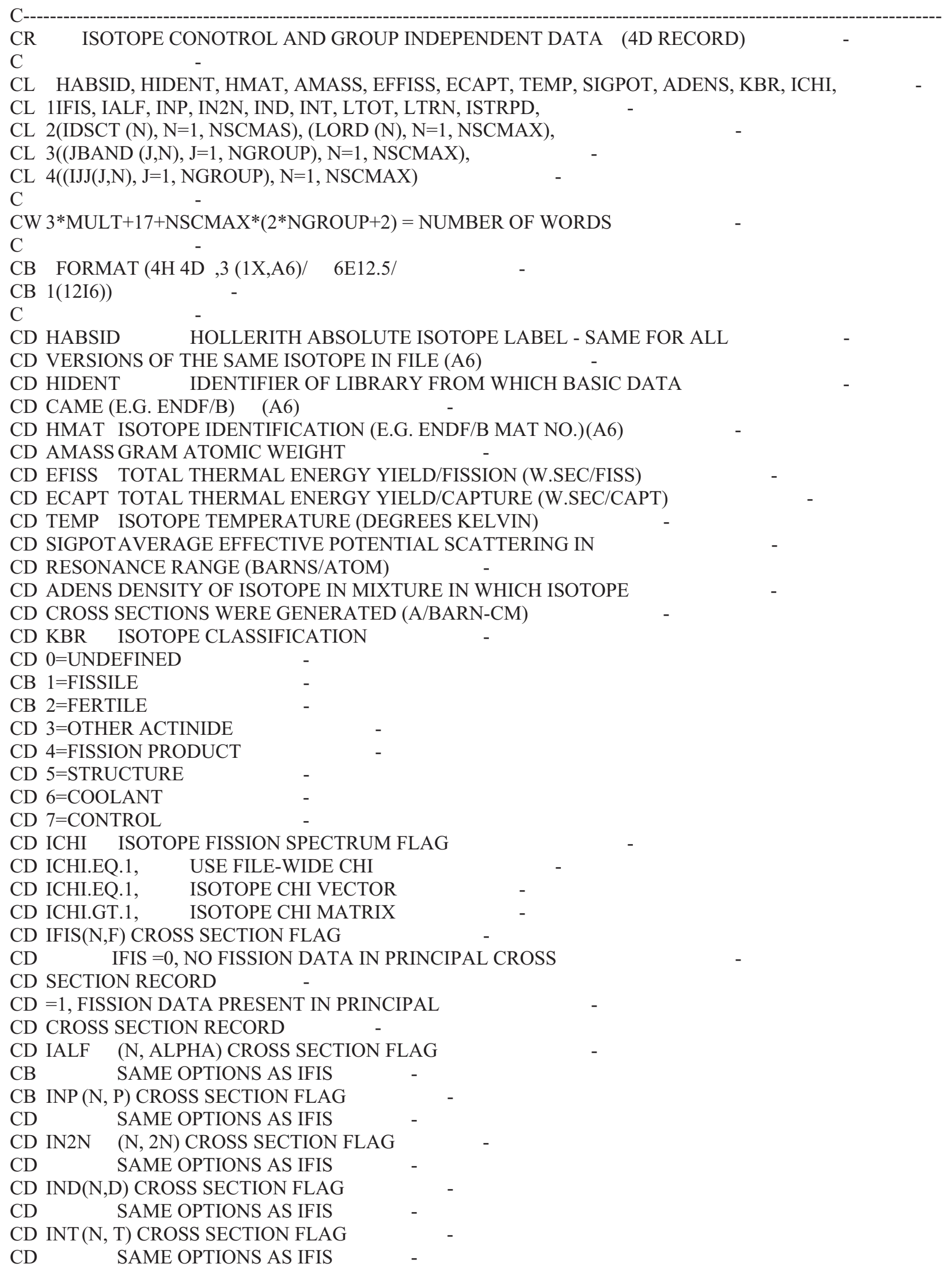




\begin{tabular}{|c|c|c|c|c|c|}
\hline $\mathrm{CD}$ & LTOT & NUMBER OF MOMENTS OF TOTAL CROSS SECTION PROVIDEI & & - & \\
\hline $\mathrm{CD}$ & & IN PRINCIPAL CROSS SECTIONS RECORD ～－ & & & \\
\hline $\mathrm{CD}$ & LTRN & NUMBER OF MOMENTS OF TRANSPORT CROSS SECTION & & - & \\
\hline $\mathrm{CD}$ & & PROVIDED IN PRINCIPAL CROSS SECTIONS RECORD & - & & \\
\hline $\mathrm{CD}$ & ISTRPD & NUMBER OF COORDINATE DIRECTIONS FOR WHICH & - & & \\
\hline $\mathrm{CD}$ & & COORDINATE DEPENDENT TRANSPORT CROSS SECTIONS & - & & \\
\hline $\mathrm{CD}$ & & ARE GIVEN. IF ISTRPD $=0$, NO COORDINATE DEPENDNET & - & & \\
\hline $\mathrm{CD}$ & & TRANSPORT CROSS SECTIONS ARE GIVEN. & & & \\
\hline $\mathrm{CB}$ & IDSCT(N & N) SCATTERING MATRIX TYPE IDENTIFICATION FOR & & - & \\
\hline $\mathrm{CB}$ & & SCATTERING BLOCK N. SIGNIFICANT ONLY IF & - & & \\
\hline $\mathrm{CD}$ & & LORD (N). GT.0 $\quad-$ & & & \\
\hline $\mathrm{CD}$ & & IDSCT $(\mathrm{N})=000+\mathrm{NN}$, TOTAL SCATTERING, (SUM OF & - & & \\
\hline $\mathrm{CD}$ & ELASTIC & IC, INELASTIC, AND N, 2N SCATTERING - & & & \\
\hline & MATRIX & X TERMS). & & & \\
\hline $\mathrm{CD}$ & $=100$ & $0+$ + NN, ELASTIC SCATTERING & & & \\
\hline $\mathrm{CD}$ & $=200$ & $0+\mathrm{NN}$, INELASTIC SCATTERING & & & \\
\hline $\mathrm{CD}$ & $=300$ & $0+\mathrm{NN},(\mathrm{N}, 2 \mathrm{~N}) \mathrm{SCATTERING,-----SEE}$ & & & \\
\hline $\mathrm{CD}$ & & OTE BELOW & & & \\
\hline $\mathrm{CD}$ & & WHERE NN IS THE LEGENDRE EXPANSION INDEX OF THE & - & & \\
\hline $\mathrm{CD}$ & & FIRST MATRIX IN BLOCK N ～～～- & & & \\
\hline $\mathrm{CD}$ & LORD ( & (N) NUMBER OF SCATTERING ORDERS IN BLOCK N. IF & & & - \\
\hline $\mathrm{CD}$ & & LORD $(\mathrm{N})=0$, THIS BLOCK IS NOT PRESENT FOR THIS & - & & \\
\hline $\mathrm{CD}$ & & ISOTOPE. IF NN IS HE VALUE TKEN FROM ～～- & & & \\
\hline $\mathrm{CD}$ & & IDSCT (N), THEN THE MATRICES IN THIS BLOCK & - & & \\
\hline $\mathrm{CD}$ & & HAVE LEGENDRE EXPANSION INDICES OF NN, NN+1, & - & & \\
\hline $\mathrm{CB}$ & & $\mathrm{NN}+2, \ldots, \mathrm{NN}+\mathrm{LORD}(\mathrm{N})-1$ & & & \\
\hline $\mathrm{CB}$ & JBAND $($. & $(\mathrm{J}, \mathrm{N}) \quad$ NUMBER OF GROUPS THAT SCATTER INTO GROUP J, & & - & \\
\hline $\mathrm{CD}$ & & INCLUDING SELF-SCATTER, IN SCATERING BLOCK N. & - & & \\
\hline $\mathrm{CD}$ & & IF JBAND $(\mathrm{J}, \mathrm{N})=0$, NO SCATTER DATA IS PRESENT IN & - & & \\
\hline $\mathrm{CD}$ & & BLOCK N - & & & \\
\hline $\mathrm{CD}$ & $\operatorname{IJJ}(\mathrm{J}, \mathrm{N})$ & POSITION OF IN-GROUP SCATTERING CROSS SECTION IN & - & & \\
\hline $\mathrm{CD}$ & & SCATTERING DATA FOR GROUP J, SCATTERING BLOCK & & - & \\
\hline $\mathrm{CD}$ & & N, COUNTED FROM THE FIRST WORD OF GROUP J DATA. & & - & \\
\hline $\mathrm{CD}$ & & IF JBAND (J,N). NE. 0 THEN IJJ(,N) MUST SATISFY & - & & \\
\hline $\mathrm{CD}$ & & THE RELATION 1.LE.IJJ (J,N).LE.JBAND (J,N) & & & \\
\hline $\mathrm{C}$ & & - & & & \\
\hline $\mathrm{CN}$ & NOT & TE- FOR N,2N, SCATTER, THE MATRIX CONTAINS TERMS, & & - & \\
\hline $\mathrm{CN}$ & & SCAT (J TO G), WHICH ARE EMISSION (PRODUCTION) & - & & \\
\hline $\mathrm{CN}$ & & BASED, I.E., ARE DEFINED SUCH THAT MACROSCOPIC & - & & \\
\hline $\mathrm{CN}$ & & SCAT (J TO G) TIMES THE FLUX IN GROUP J GIVES & - & & \\
\hline $\mathrm{CN}$ & & THE RATE OF EMISSION (PRODUCTION) OF NEUTRONS & - & & \\
\hline $\mathrm{CN}$ & & INTO GROUP G. & & & \\
\hline $\mathrm{C}$ & & 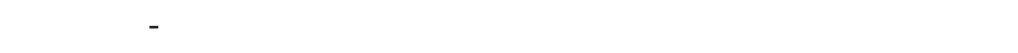 & & & \\
\hline
\end{tabular}




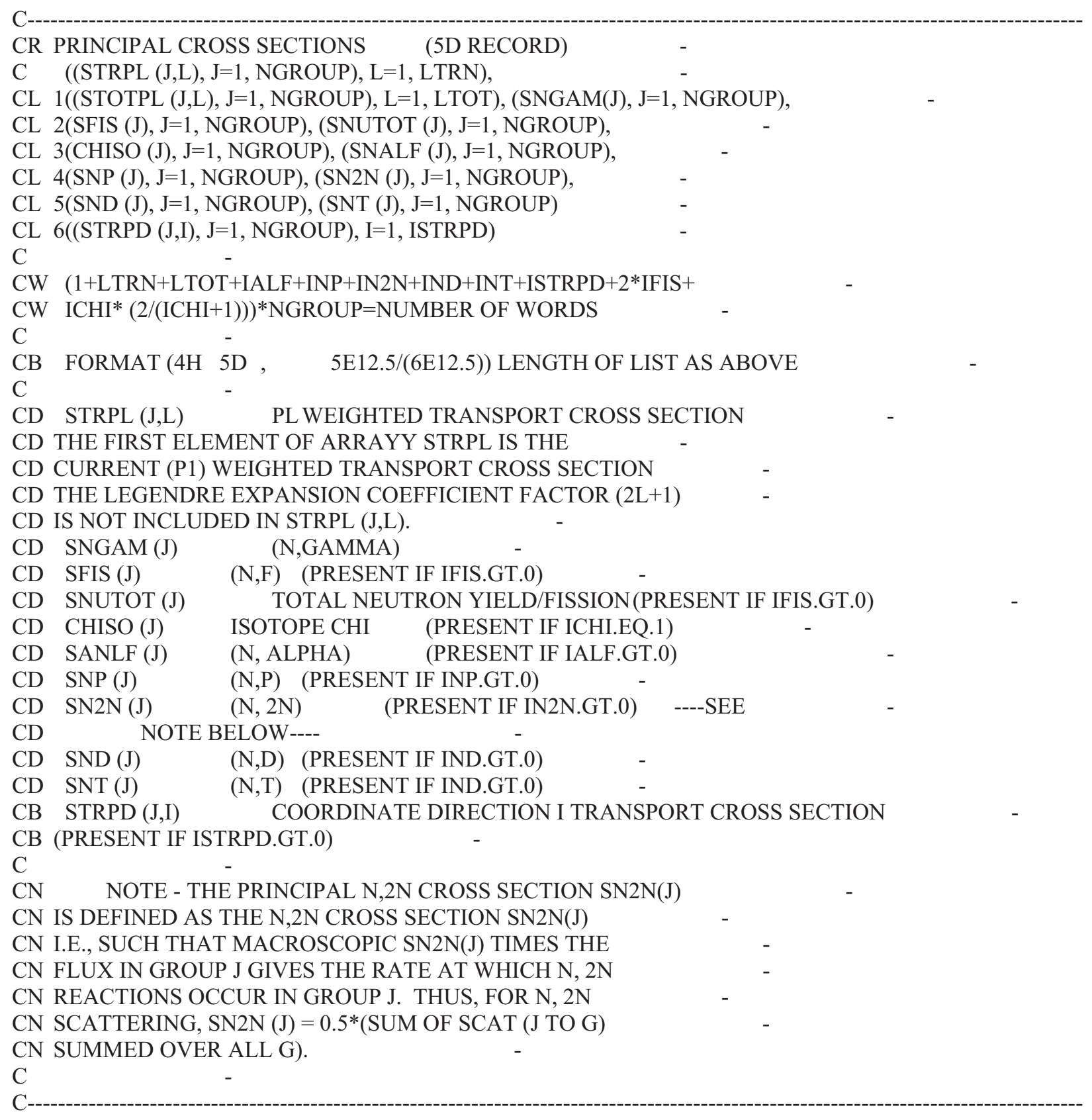




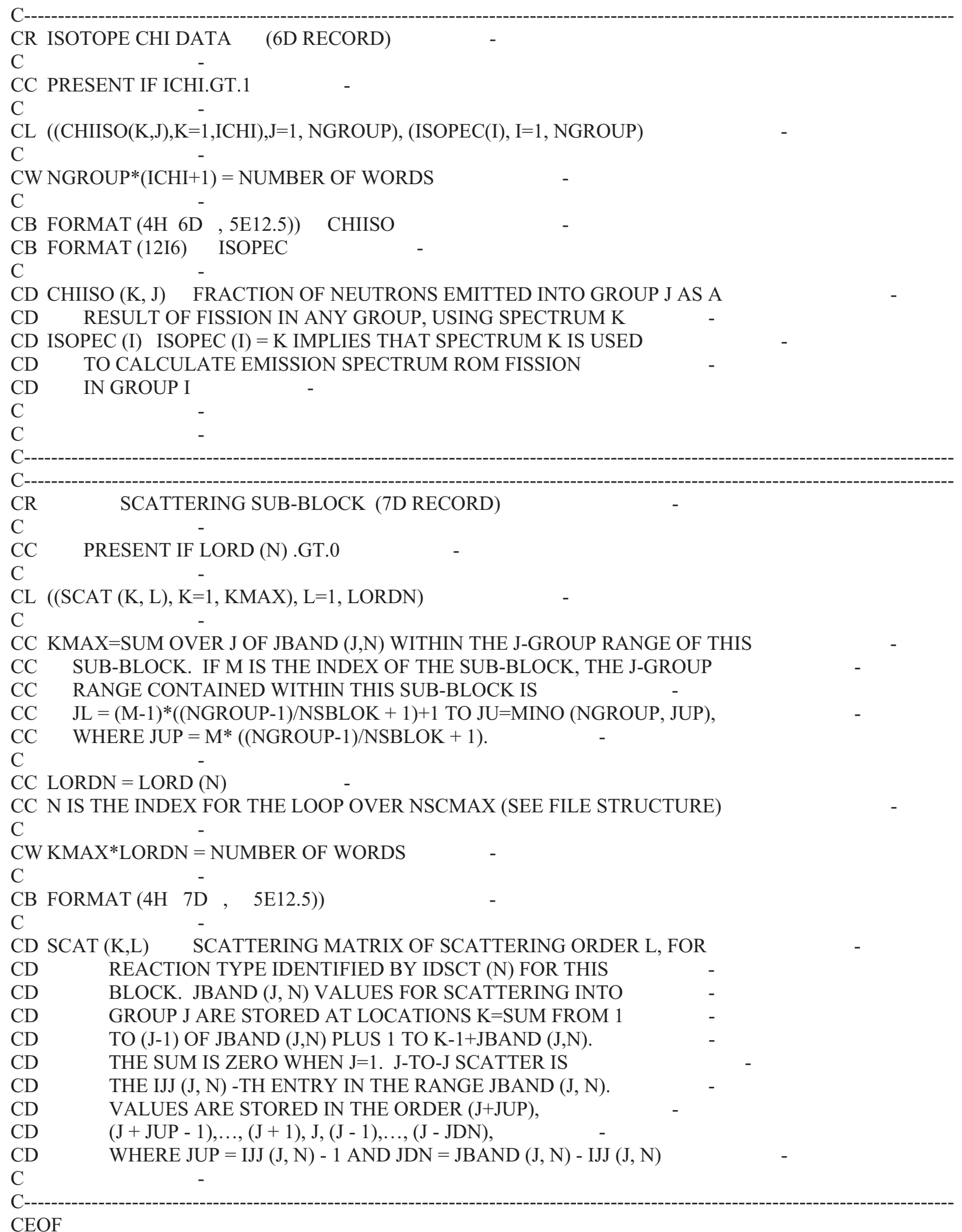




\section{APPENDIX D}

\section{VALIADTION OF COMBINE7.1 CRITICALITY SEQUENCES}




\section{Appendix D Validation of COMBINE7.1 Criticality Sequences}

Criticality validation calculations were performed as part of verification and validation of the code. The COMBINE7.1 criticality validation suite is a collection of 29 benchmarks taken from the International Handbook of Evaluated Criticality Benchmark Experiments (IHECB) [1] and/or Cross Section Evaluation Working Group Benchmark Specifications (CSEWG) [2]. The cases in the suite were selected to compass a variety of fissle materials in configurations that produce fast, intermediate, and thermal spectra, but were limited to those configurations which COMBINE7.1 calculation sequence, i.e., COMBINE7.1/ANISN, can afford,. Some independent MCNP [3] calculations were also performed, all based on ENDF/B-VII.0 nuclear data. The distribution of cases by fuel material and spectrum is shown in Table D.1, and a brief summary of each case is followed. The results are listed in Table D.2, D.3, D.4, and D.5 for the combination of B1 or B3 and Sn correction or no Sn correction. The experiment $k_{\text {eff }}$ values have some uncertaites involved and estimated to be mostly with in $1.0000 \pm 0.0050$ [1]. The $k_{\text {eff }}$ values from MCNP calculations are also included. The results show the sensitivity to the deterministic nature of the method and to the $B_{L}$ approximation limited to $\mathrm{L}=1$ or $\mathrm{L}=3$. The results are very robust despite these limitations and are very comparable to the experiments or the MCNP results. A limited number of cases included the one-dimensional transport correction, showing a small improvement. Some sensitivity can be digested from these calculations. All the relevant input files are included in the code package.

Table D.1: Criticality Validation Suite

\begin{tabular}{|c|c|c|c|c|c|c|c|}
\hline \multirow[t]{2}{*}{ Spectrum } & \multicolumn{3}{|c|}{ Fast } & \multicolumn{2}{|l|}{ Intermediate } & \multicolumn{2}{|l|}{ Thermal } \\
\hline & & Heavy & Light & & Lattice of Fuel & Sout & \\
\hline \multirow{5}{*}{$\begin{array}{c}\text { Geometry } \\
\text { HEU }\end{array}$} & Bare & Reflector & Refelctor & Any & Pins in Water & Bare & Reflector \\
\hline & GODIVA & FLATTOP-25 & & HCI-004 & & ORNL-1, & L-7, \\
\hline & & & & & & ORNL-2, & L-10 \\
\hline & & & & & & ORNL-10, & \\
\hline & & & & & & L-8, L-9 & \\
\hline IEU & IEU-MF-03 & & & & & & \\
\hline \multirow[t]{2}{*}{ LEU } & & & & & TRX1, TRX2, & & \\
\hline & & & & & $\begin{array}{l}\text { BAPL1, } \\
\text { BAPL2, } \\
\text { BAPL3 }\end{array}$ & & \\
\hline \multirow[t]{2}{*}{${ }^{233} \mathrm{U}$} & JEZEBEL-23 & UMF002-1, & UMF005-1, & & & & \\
\hline & & UMF002-2 & UMF005-2 & & & & \\
\hline $\mathrm{Pu}$ & $\begin{array}{l}\text { JEZEBEL, } \\
\text { JEZEBEL-Pu }\end{array}$ & $\begin{array}{c}\text { FLATTOP- } \\
\text { PU }\end{array}$ & & PCI-001 & & $\begin{array}{l}\text { PNL-1, PNL- } \\
\text { 2, PNL-3, } \\
\text { PNL-4 }\end{array}$ & \\
\hline
\end{tabular}

\section{GODIVA}

This is a bare sphere of enriched uranium metal modeled as a homogeneous sphere of radius 8.741 $\mathrm{cm}$ with the composition shown in the following table (number densities are given in nuclides per barn$\mathrm{cm})$.

\begin{tabular}{|c|c|}
\hline Isotope & Density \\
\hline${ }^{235} \mathrm{U}$ & 0.045000 \\
\hline${ }^{238} \mathrm{U}$ & 0.002498 \\
\hline${ }^{234} \mathrm{U}$ & 0.000492 \\
\hline
\end{tabular}




\section{FLATTOP-25}

This is a spherical system consisting of a highly enriched uranium core in a thick natural uranium reflector. The core radius is $6.116 \mathrm{~cm}$ and the outer radius of the reflector is $24.13 \mathrm{~cm}$. The compositions for the two regions (nuclides per bar-cm) are given in the following table.

\begin{tabular}{|c|c|c|}
\hline Isotope & Core & Reflector \\
\cline { 1 - 1 }${ }^{235} \mathrm{U}$ & 0.04449 & 0.00034 \\
\cline { 1 - 1 }${ }^{238} \mathrm{U}$ & 0.00270 & 0.04774 \\
\cline { 1 - 1 }${ }^{234} \mathrm{U}$ & 0.00049 & \\
\hline
\end{tabular}

\section{FLATTOP-PU}

This is a spherical system consiting of a plutonium core in a thick natural uranium reflector. The radius of the core is $4.533 \mathrm{~cm}$ and the outer radius of the reflector is $24.13 \mathrm{~cm}$. The compositions of the two regions are given in the following table.

\begin{tabular}{|c|c|c|}
\hline Isotope & Core & Reflector \\
\hline${ }^{239} \mathrm{Pu}$ & 0.03674 & \\
\cline { 1 - 2 }${ }^{240} \mathrm{Pu}$ & 0.00186 & \\
\cline { 1 - 2 }${ }^{241} \mathrm{Pu}$ & 0.00012 & \\
\cline { 1 - 2 } $\mathrm{Ga}$ & 0.00138 & \\
\cline { 1 - 1 }${ }^{235} \mathrm{U}$ & & 0.00034 \\
\cline { 1 - 1 }${ }^{238} \mathrm{U}$ & & 0.04774 \\
\hline
\end{tabular}

\section{JEZEBEL}

This is a bare sphere of plutonium modeled as a homogeneous sphere of radius $6.385 \mathrm{~cm}$ with the composition shown in the following table (all number of densities are given as nuclides per barn-cm).

\begin{tabular}{|c|c|}
\hline Isotope & Density \\
\hline${ }^{239} \mathrm{Pu}$ & 0.037050 \\
\hline${ }^{240} \mathrm{Pu}$ & 0.001751 \\
\hline${ }^{241} \mathrm{Pu}$ & 0.000117 \\
\hline $\mathrm{Ga}$ & 0.001375 \\
\hline
\end{tabular}

\section{JEZEBEL-23}

This is a bare sphere of enriched ${ }^{233} \mathrm{U}$ metal $(98.13 \%)$ with radius $5.983 \mathrm{~cm}$ and the composition shown in the following table (all number of densities are given as nuclides per barn-cm).

\begin{tabular}{|c|c|}
\hline Isotope & Density \\
\hline${ }^{233} \mathrm{U}$ & 0.04671 \\
\hline${ }^{234} \mathrm{U}$ & 0.00059 \\
\hline${ }^{235} \mathrm{U}$ & 0.00001 \\
\hline${ }^{238} \mathrm{U}$ & 0.00029 \\
\hline
\end{tabular}




\section{JEZEBEL-Pu}

This is a bare sphere of enriched plutonium meta, containing $20.1 \%{ }^{240} \mathrm{Pu}$. It is modeled as a homogeneous sphere of radius $6.65985 \mathrm{~cm}$ with the composition shown in the following table (all number of densities are given as nuclides per barn-cm).

\begin{tabular}{|c|c|}
\hline Isotope & Density \\
\hline${ }^{239} \mathrm{Pu}$ & 0.029946 \\
\hline${ }^{240} \mathrm{Pu}$ & 0.007887 \\
\hline${ }^{241} \mathrm{Pu}$ & 0.001203 \\
\hline${ }^{242} \mathrm{Pu}$ & 0.000145 \\
\hline $\mathrm{Ga}$ & 0.001372 \\
\hline
\end{tabular}

\section{U233-met-fast-002 (umf-002)}

This is a spherical system consisting of a highly enriched ${ }^{233} \mathrm{U}$ surrounded by highly enriched ${ }^{235} \mathrm{U}$.

- Case (1) The core radius is $5.0444 \mathrm{~cm}$ and the outer radius of the surrounding highly enriched ${ }^{235} \mathrm{U}$ is $6.2661 \mathrm{~cm}$.

- Case (2) The core radius is $4.5999 \mathrm{~cm}$ and the outer radius of the surrounding highly enriched ${ }^{235} \mathrm{U}$ is $6.5887 \mathrm{~cm}$.

Both critical experiments were performed at Los Alamos Scientific Laboratory.

The compositions for the two regions (nuclides per bar-cm) of these cases are given in the following table. Temperature is at $293{ }^{0} \mathrm{~K}$.

\begin{tabular}{|c|c|c|}
\hline Isotope & Case 1 & Case 2 \\
\hline \multicolumn{3}{|c|}{${ }^{233} \mathrm{U}$ core } \\
\hline${ }^{233} \mathrm{U}$ & $4.7253 \times 10^{-2}$ & $4.7312 \times 10^{-2}$ \\
\hline${ }^{234} \mathrm{U}$ & $5.2705 \times 10^{-4}$ & $5.2770 \times 10^{-4}$ \\
\hline${ }^{238} \mathrm{U}$ & $3.2975 \times 10^{-4}$ & $3.3015 \times 10^{-4}$ \\
\hline \multicolumn{3}{|c|}{${ }^{235} \mathrm{U}$ Surroundings } \\
\hline${ }^{235} \mathrm{U}$ & $4.4892 \times 10^{-1}$ & $4.4892 \times 10^{-1}$ \\
\hline${ }^{238} \mathrm{U}$ & $3.2340 \times 10^{-3}$ & $3.2340 \times 10^{-3}$ \\
\hline
\end{tabular}

\section{U233-met-fast-005 (umf-005)}

This is a spherical system consisting of a highly enriched ${ }^{233} \mathrm{U}$, reflected by beryllium.

- Case (1) The core radius is $5.0444 \mathrm{~cm}$ and the outer radius of the reflector is $7.0891 \mathrm{~cm}$.

- Case (2) The core radius is $4.5999 \mathrm{~cm}$ and the outer radius of the reflector is $8.7960 \mathrm{~cm}$. Both critical experiments were performed at Los Alamos Scientific Laboratory. 
The compositions for the two regions (nuclides per bar-cm) of these cases are given in the following table. Temperature is at $293{ }^{0} \mathrm{~K}$.

\begin{tabular}{|c|c|c|}
\hline Isotope & Case 1 & Case 2 \\
\cline { 1 - 2 } \multicolumn{3}{|c|}{${ }^{233} \mathrm{U}$ core } \\
\cline { 1 - 2 }${ }^{233} \mathrm{U}$ & $4.7253 \times 10^{-2}$ & $4.7312 \times 10^{-2}$ \\
\cline { 1 - 1 }${ }^{234} \mathrm{U}$ & $5.2705 \times 10^{-4}$ & $5.2770 \times 10^{-4}$ \\
\cline { 1 - 1 }${ }^{238} \mathrm{U}$ & $3.2975 \times 10^{-4}$ & $3.3015 \times 10^{-4}$ \\
\cline { 1 - 2 } & \multicolumn{3}{|c|}{ Beryllium Reflector } \\
\cline { 1 - 1 } $\mathrm{Be}$ & $1.1984 \times 10^{-1}$ & $1.1984 \times 10^{-1}$ \\
\cline { 1 - 1 } $\mathrm{O}$ & $1.3776 \times 10^{-3}$ & $1.3776 \times 10^{-3}$ \\
\hline
\end{tabular}

\section{Pu-Comp-Inter-001 (PCI-001)}

This is a $k_{\infty}$ experiment at the HECTOR reactor (Winfrith, UK, 1960s): a graphite moderated plutonium oxide core $\left(5 \%{ }^{240} \mathrm{Pu}\right)$. The benchmark model is an infinite medium with a material composition appropriate to the interpolated boron $/{ }^{239} \mathrm{Pu}$ ratio. It is modeled as a homogeneous infinite medium with the composition shown in the following table (all number of densities are given as nuclides per barn-cm).

\begin{tabular}{|c|c|}
\hline Isotope & Density \\
\hline${ }^{239} \mathrm{Pu}$ & $2.7350 \times 10^{-4}$ \\
\hline${ }^{240} \mathrm{Pu}$ & $1.5490 \times 10^{-5}$ \\
\hline${ }^{241} \mathrm{Pu}$ & $1.0720 \times 10^{-6}$ \\
\hline${ }^{242} \mathrm{Pu}$ & $5.8000 \times 10^{-8}$ \\
\hline${ }^{1} \mathrm{H}$ & $1.0770 \times 10^{-4}$ \\
\hline${ }^{10} \mathrm{~B}$ & $1.0151 \times 10^{-4}$ \\
\hline${ }^{11} \mathrm{~B}$ & $4.0859 \times 10^{-4}$ \\
\hline $\mathrm{C}$ & $7.0900 \times 10^{-2}$ \\
\hline${ }^{16} \mathrm{O}$ & $2.7070 \times 10^{-3}$ \\
\hline${ }^{40} \mathrm{Ca}$ & $8.0267 \times 10^{-4}$ \\
\hline${ }^{42} \mathrm{Ca}$ & $5.3572 \times 10^{-6}$ \\
\hline${ }^{43} \mathrm{Ca}$ & $1.1178 \times 10^{-6}$ \\
\hline${ }^{44} \mathrm{Ca}$ & $1.7272 \times 10^{-5}$ \\
\hline${ }^{46} \mathrm{Ca}$ & $3.3120 \times 10^{-8}$ \\
\hline${ }^{48} \mathrm{Ca}$ & $1.5484 \times 10^{-6}$ \\
\hline
\end{tabular}




\section{HEU-Comp-Inter-004 (HCI-004)}

This is a $k_{\infty}$ experiment at the HECTOR reactor (Winfrith, UK, 1960s): a graphite moderated uranium oxide core. The benchmark model is an infinite medium with a material composition appropriate to the interpolated boron $/{ }^{235} \mathrm{U}$ ratio. It is modeled as a homogeneous infinite medium with the composition shown in the following table (all number of densities are given as nuclides per barn-cm).

\begin{tabular}{|c|c|}
\hline Isotope & Density \\
\hline${ }^{234} \mathrm{U}$ & $3.1200 \times 10^{-6}$ \\
\hline${ }^{235} \mathrm{U}$ & $2.5020 \times 10^{-4}$ \\
\hline${ }^{236} \mathrm{U}$ & $4.2670 \times 10^{-7}$ \\
\hline${ }^{238} \mathrm{U}$ & $1.7190 \times 10^{-5}$ \\
\hline${ }^{1} \mathrm{H}$ & $1.1260 \times 10^{-4}$ \\
\hline${ }^{10} \mathrm{~B}$ & $5.7849 \times 10^{-5}$ \\
\hline${ }^{11} \mathrm{~B}$ & $2.3285 \times 10^{-4}$ \\
\hline $\mathrm{C}$ & $7.5650 \times 10^{-2}$ \\
\hline${ }^{16} \mathrm{O}$ & $1.6500 \times 10^{-2}$ \\
\hline${ }^{40} \mathrm{Ca}$ & $5.5227 \times 10^{-4}$ \\
\hline${ }^{42} \mathrm{Ca}$ & $3.6860 \times 10^{-6}$ \\
\hline${ }^{43} \mathrm{Ca}$ & $7.6909 \times 10^{-7}$ \\
\hline${ }^{44} \mathrm{Ca}$ & $1.1884 \times 10^{-5}$ \\
\hline${ }^{46} \mathrm{Ca}$ & $2.2788 \times 10^{-8}$ \\
\hline${ }^{48} \mathrm{Ca}$ & $1.0653 \times 10^{-6}$ \\
\hline
\end{tabular}

\section{AQUEOUS THERML CRITICALS}

Three unreflected spheres from the ORNL series were computed. These benchmarks contain ${ }^{235} \mathrm{U}$ in the form of uranyl nitrate dissolved in water. One of the devices was poisoned with boron. The CSEWG treatment for these criticals models them as bare spheres of the core solution corrected for the thin aluminum shells, nonspherical shape, fill tubes, and room retrun. Benchmarks ORNL-1 and ORNL-2 are of radius $34.595 \mathrm{~cm}$, and ORNL-10 has a radius of $61.011 \mathrm{~cm}$. The compositions specified for the CSEWG models are as follows.

\begin{tabular}{|c|c|c|c|}
\hline Material & ORNL-1 & ORNL-2 & ORNL-10 \\
\hline${ }^{10} \mathrm{~B}$ & 0.0 & $1.0286 \times 10^{-6}$ & 0.0 \\
\hline $\mathrm{H}$ & 0.066228 & 0.066148 & 0.066394 \\
\hline $\mathrm{O}$ & 0.033736 & 0.033800 & 0.033592 \\
\hline $\mathrm{N}$ & $1.869 \times 10^{-4}$ & $2.129 \times 10^{-4}$ & $1.116 \times 10^{-4}$ \\
\hline${ }^{234} \mathrm{U}$ & $5.38 \times 10^{-7}$ & $6.31 \times 10^{-7}$ & $4.09 \times 10^{-7}$ \\
\hline${ }^{235} \mathrm{U}$ & $4.8066 \times 10^{-5}$ & $5.6206 \times 10^{-5}$ & $3.6185 \times 10^{-5}$ \\
\hline${ }^{236} \mathrm{U}$ & $1.38 \times 10^{-7}$ & $1.63 \times 10^{-7}$ & $2.20 \times 10^{-7}$ \\
\hline${ }^{238} \mathrm{U}$ & $2.807 \times 10^{-6}$ & $3.281 \times 10^{-6}$ & $1.985 \times 10^{-6}$ \\
\hline
\end{tabular}


In order to get a case with higher leakage, one additional sphere from the L series of uranyl fluoride assemblies was calculated. The L-7 assembly is modeled as an aluminum-clad sphere with radius of $11.5176 \mathrm{~cm}$, respectively. The aluminum container has a thickness of 0.16 , and the aluminum number density is taken to be 0.06025 per barn-cm. The number densities for the solution are given in the following table.

\begin{tabular}{|c|c|c|c|c|c|}
\hline Material & L-7 & L-8 & L-9 & L-10 & L-11 \\
\hline $\mathrm{H}$ & 0.0633680 & 0.0666144 & 0.066634 & 0.0647289 & 0.066722 \\
\hline $\mathrm{O}$ & 0.0334713 & 0.0334357 & 0.0334344 & 0.034627 & 0.033473 \\
\hline $\mathrm{F}$ & 0.0017873 & 0.00012855 & 0.0001027 & 0.0010983 & 0.00011249 \\
\hline${ }^{234} \mathrm{U}$ & $8.7577 \times 10^{-6}$ & $7.327 \times 10^{-7}$ & $5.854 \times 10^{-7}$ & $5.3816 \times 10^{-6}$ & $5.5393 \times 10^{-7}$ \\
\hline${ }^{235} \mathrm{U}$ & $8.32694 \times 10^{-4}$ & $5.99051 \times 10^{-5}$ & $4.7856 \times 10^{-5}$ & $5.11691 \times 10^{-4}$ & $5.2444 \times 10^{-5}$ \\
\hline${ }^{236} \mathrm{U}$ & $4.2895 \times 10^{-6}$ & 0.0 & 0.0 & $2.6359 \times 10^{-6}$ & $2.8022 \times 10^{-7}$ \\
\hline${ }^{238} \mathrm{U}$ & $4.78991 \times 10^{-5}$ & $3.638 \times 10^{-6}$ & $2.9063 \times 10^{-6}$ & $2.9340 \times 10^{-5}$ & $2.9675 \times 10^{-6}$ \\
\hline
\end{tabular}

The L-7, L-10, and L-11 assembly has an additional $20.0 \mathrm{~cm}$ of water outside the aluminum shell (the number densities for the hydrogen and oxygen are .0666883 and .0333442 per bar-cm, respectively).

Four unreflected spheres of plutonium nitrate solution were also computed. The PNL-1 and PNL-2 benchmarks both have an effective radius of $19.509 \mathrm{~cm}$. The isotopic compositions of the solutions are given in the following table.

\begin{tabular}{|c|c|c|}
\hline Material & PNL-1 & PNL-2 \\
\hline $\mathrm{H}$ & 0.06563 & 0.05416 \\
\hline $\mathrm{O}$ & 0.03456 & 0.03977 \\
\hline $\mathrm{N}$ & $6.216 \times 10^{-4}$ & $4.720 \times 10^{-3}$ \\
\hline${ }^{239} \mathrm{Pu}$ & $9.373 \times 10^{-5}$ & $4.141 \times 10^{-4}$ \\
\hline${ }^{240} \mathrm{Pu}$ & $4.501 \times 10^{-6}$ & $1.988 \times 10^{-5}$ \\
\hline
\end{tabular}

Similarly, the PNL-3 and PNL-4 benchmarks have an effective radius of $22.70 \mathrm{~cm}$. The isotopic compositions of the solutions are given in the following table.

\begin{tabular}{|c|c|c|}
\hline Material & PNL-1 & PNL-2 \\
\hline $\mathrm{H}$ & 0.06495 & 0.06041 \\
\hline $\mathrm{O}$ & 0.03441 & 0.03712 \\
\hline $\mathrm{N}$ & $7.394 \times 10^{-4}$ & $2.775 \times 10^{-3}$ \\
\hline $\mathrm{Fe}$ & $1.294 \times 10^{-6}$ & $1.520 \times 10^{-6}$ \\
\hline${ }^{239} \mathrm{Pu}$ & $5.395 \times 10^{-5}$ & $6.633 \times 10^{-5}$ \\
\hline${ }^{240} \mathrm{Pu}$ & $2.355 \times 10^{-6}$ & $2.895 \times 10^{-6}$ \\
\hline
\end{tabular}




\section{LATTICE THERMAL CRITICALS TRX LATTICES}

The TRX lattices were a series using a triangular pattern of slightly-enriched metallic uranium rods in a water moderator. The CSEWG benchmarks model the unit cell with cylindrical geometry as given in the following table.

\begin{tabular}{|c|c|c|c|}
\hline Region & Radius & Isotope & Density \\
\hline Fuel & 0.4915 & ${ }^{235} \mathrm{U}$ & $6.253 \times 10^{-4}$ \\
\hline & & ${ }^{238} \mathrm{U}$ & $4.7205 \times 10^{-2}$ \\
\hline Void & 0.5042 & & \\
\hline Clad & 0.5753 & $\mathrm{Al}$ & $6.025 \times 10^{-2}$ \\
\hline Moderator & $*$ & ${ }^{1} \mathrm{H}$ & $6.676 \times 10^{-2}$ \\
\hline & & ${ }^{16} \mathrm{O}$ & $3.338 \times 10^{-2}$ \\
\hline
\end{tabular}

The $*$ in the table represents the outer radius of the moderator region, which varies between the two lattices analyzed here. The lattice spacings for TRX-1 and TRX-2 are $1.8060 \mathrm{~cm}$ and $2.1740 \mathrm{~cm}$, respectively, which translate into radii of $0.9482 \mathrm{~cm}$ and $1.1414 \mathrm{~cm}$, respectively.

The specifications in ENDF-202 sugest that these assemblies can be computed as infinite cylinders, and then a lekage correction can be applied using an homogenized $B_{l}$ calculation with a total buckling of $B^{2}=0.0057 \mathrm{~cm}^{-2}$ for TRX-1 or $B^{2}=0.005469 \mathrm{~cm}^{-2}$ for TRX-2. He physical height is approximately $136.98 \mathrm{~cm}$ with vacuum boundary conditions. TRX-1 contained 764 fuel rods. When circularized, this corresponds to an effective radius of $26.209 \mathrm{~cm}$. TRX-2 contained 578 fuel rods. The corresponding radius for TRX-2 is $27.441 \mathrm{~cm}$. The water reflector was taken to have a thickness of about $30 \mathrm{~cm}$ with a vacuum boundary condition on its outer surface.

\section{BAPL LATTICES}

The BAPL lattices were a series using a triangular pattern of slightly-enriched uranium oxide rods in a water moderator. The CSEWG benchmarks model the unit cell with cylindrical geometry as given in the following table.

\begin{tabular}{|c|c|c|c|}
\hline Region & Radius & Isotope & Density \\
\hline Fuel & 0.4864 & ${ }^{235} \mathrm{U}$ & $3.112 \times 10^{-4}$ \\
\hline & & ${ }^{238} \mathrm{U}$ & $2.3127 \times 10^{-2}$ \\
\hline & & ${ }^{16} \mathrm{O}$ & $4.6946 \times 10^{-2}$ \\
\hline Void & 0.5042 & & \\
\hline Clad & 0.5753 & $\mathrm{Al}$ & $6.025 \times 10^{-2}$ \\
\hline Moderator & $*$ & ${ }^{1} \mathrm{H}$ & $6.676 \times 10^{-2}$ \\
\hline & & ${ }^{16} \mathrm{O}$ & $3.338 \times 10^{-2}$ \\
\hline
\end{tabular}

The $*$ in the table represents the outer radius of the moderator region, which varies between the three lattices analyzed here. The lattice spacings for BAPL-1, BAPL-2, and BAPL-3 are 1.5578, 1.6523, and $1.8057 \mathrm{~cm}$, respectively, which translate into radii of $0.8178,0.8675$, and $0.9480 \mathrm{~cm}$, respectively.

These three lattices can be calculated using the methods discussed above for the TRX lattices. The axial buckling is taken to be the same as for the TRX lattices; namely, 0.000526, giving a buckling height of $136.98 \mathrm{~cm}$. The core radii can be calculated from the cell radii and the critical number of rods: 38.122 $\mathrm{cm}$ for BAPL-1 (2173 rods), $36.342 \mathrm{~cm}$ for BAPL-2 (1755-rods), and $37.623 \mathrm{~cm}$ for BAPL-3 (1575 rods). 
Table D.2: Calculated keff eigenvalues against the measured keff $=1.000$ B1 and No Sn Transport Correction

\begin{tabular}{|c|c|c|c|}
\hline \multirow[b]{3}{*}{$\begin{array}{c}\text { IHECB/CSEWG } \\
\text { Benchmarks } \\
\end{array}$} & \multicolumn{3}{|c|}{$\boldsymbol{k}_{\text {eff }}$} \\
\hline & & \multicolumn{2}{|c|}{ COMBINE7.1 } \\
\hline & menp & $\begin{array}{l}\text { Bondarenko } \\
\text { Self shielding }\end{array}$ & $\begin{array}{c}\text { Bondarenko + } \\
\text { Nordheim } \\
\text { self shielding } \\
\end{array}$ \\
\hline GODIVA (hmf-001) & $1.00014(22)$ & 0.99797 & 0.99797 \\
\hline FLATTOP-25 (hmf-028) & $1.00275(24)$ & 0.99900 & 0.99900 \\
\hline FLATTOP-Pu (pmf-006) & $1.00067(25)$ & 0.99325 & 0.99326 \\
\hline JEZEBEL (pmf-001 bare) & $1.0001(20)$ & 0.99612 & 0.99612 \\
\hline JEZEBEL-23 (umf-001) & $1.00032(21)$ & 0.99647 & 0.99647 \\
\hline JEZEBEL-Pu (pmf-002) & $1.00025(20)$ & 0.99665 & 0.99665 \\
\hline umf002-1 & $0.99891(21)$ & $0.99458 / 0.99408$ & $0.99458 / 0.99408$ \\
\hline umf002-2 & $1.00002(21)$ & $0.99542 / 0.99477$ & $0.99542 / 0.99477$ \\
\hline umf005-1 & $0.99396(21)$ & $0.98844 / 0.98801$ & $0.98769 / 0.98801$ \\
\hline umf005-2 & $0.99201(23)$ & $0.98201 / 0.98519$ & $0.98201 / 0.98519$ \\
\hline pci-001 & $1.01150(28)$ & 1.00991 & 1.00497 \\
\hline hci-004 & $0.98781(24)$ & 0.99223 & 0.98942 \\
\hline ORNL-1 (hst-013 Case 1) & & 0.99806 & 0.99794 \\
\hline ORNL-2 (hst-013 Case 2) & & 0.99717 & 0.99704 \\
\hline ORNL-10 (hst-032) & & 0.99133 & 0.99903 \\
\hline L-7 (hst009-3) & & 0.99835 & 0.99745 \\
\hline L-8 & & 1.00495 & 1.00488 \\
\hline L-9 & & 1.00292 & 1.00288 \\
\hline L-10 & & 0.99573 & 0.99521 \\
\hline L-11 (hst012) & & 1.00021 & 1.00016 \\
\hline PNL-1 & & 1.00765 & 1.00723 \\
\hline PNL-2 & & 1.00446 & 1.00391 \\
\hline PNL-3 & & 0.99398 & 0.99371 \\
\hline PNL-4 & & 0.99976 & 0.99945 \\
\hline TRX1-r ${ }^{*}$ & $0.99908(29)$ & 1.00196 & 0.99839 \\
\hline TRX2-r ${ }^{* *}$ & $0.99908(28)$ & 1.00044 & 1.00257 \\
\hline BAPL1-r ${ }^{* *}$ & & 1.00436 & 1.00367 \\
\hline BAPL2-r ${ }^{* *}$ & & 1.00136 & 1.00134 \\
\hline BAPL3-r ${ }^{* *}$ & & 1.00258 & 1.00271 \\
\hline
\end{tabular}

Note: /1-D transport correction 
Table D.3: Calculated keff eigenvalues against the measured keff $=1.000 \mathrm{~B} 1$ and Sn Transport Correction

\begin{tabular}{|c|c|c|c|}
\hline \multirow{4}{*}{$\begin{array}{c}\text { IHECB/CSEWG } \\
\text { Benchmarks }\end{array}$} & \multicolumn{3}{|c|}{$k_{\text {eff }}$} \\
\hline & \multirow[b]{3}{*}{ menp } & \multicolumn{2}{|c|}{ COMBINE7.1 } \\
\hline & & Bondarenko & Bondarenko + Nordheim \\
\hline & & Self shielding & self shielding \\
\hline GODIVA (hmf-001) & $1.00014(22)$ & 1.000190 & 1.000190 \\
\hline FLATTOP-25 (hmf-028) & $1.00275(24)$ & 0.999517 & 0.999518 \\
\hline FLATTOP-Pu (pmf-006) & $1.00067(25)$ & 0.994095 & 0.994097 \\
\hline JEZEBEL (pmf-001 bare) & $1.0001(20)$ & 0.999222 & 0.999223 \\
\hline JEZEBEL-23 (umf-001) & $1.00032(21)$ & 0.999314 & 0.999314 \\
\hline JEZEBEL-Pu (pmf-002) & $1.00025(20)$ & 0.999826 & 0.999828 \\
\hline umf002-1 & $0.99891(21)$ & $0.99865 / 0.99815$ & $0.998647 / 0.99815$ \\
\hline umf002-2 & $1.00002(21)$ & $0.99999 / 0.99935$ & $0.999991 / 0.99935$ \\
\hline umf005-1 & $0.99396(21)$ & $0.99114 / 0.99113$ & $0.990633 / 0.99113$ \\
\hline umf005-2 & $0.99201(23)$ & $0.98479 / 0.98884$ & $0.984780 / 0.98879$ \\
\hline pci-001 & $1.01150(28)$ & 1.00991 & 1.00497 \\
\hline hci-004 & $0.98781(24)$ & 0.99223 & 0.98936 \\
\hline ORNL-1 (hst-013 Case 1) & & 0.99699 & 0.99685 \\
\hline ORNL-2 (hst-013 Case 2) & & 0.99610 & 0.99594 \\
\hline ORNL-10 (hst-032) & & 0.99848 & 0.99849 \\
\hline L-7 (hst009-3) & & 0.99712 & 0.99638 \\
\hline L-8 & & 1.00374 & 1.00364 \\
\hline L-9 & & 1.00190 & 1.00182 \\
\hline $\mathrm{L}-10$ & & 0.99446 & 0.99400 \\
\hline L-11 (hst012) & & 0.99914 & 0.99906 \\
\hline PNL-1 & & 1.00610 & 1.00570 \\
\hline PNL-2 & & 1.00218 & 1.00173 \\
\hline PNL-3 & & 0.99247 & 0.99218 \\
\hline PNL-4 & & 0.99795 & 0.99763 \\
\hline TRX1-r ${ }^{*}$ & $0.99908(29)$ & 1.00035 & 0.99698 \\
\hline TRX2-r ${ }^{* *}$ & $0.99908(28)$ & 1.00307 & 1.00132 \\
\hline BAPL1-r ${ }^{* *}$ & & 1.00262 & 1.00207 \\
\hline BAPL2-r ${ }^{* *}$ & & 0.99974 & 0.99988 \\
\hline BAPL3-r ${ }^{* *}$ & & 1.00115 & 1.00142 \\
\hline
\end{tabular}

Note: /1-D transport correction 
Table D.4: Calculated keff eigenvalues against the measured keff $=1.000$ B3 and No Sn Transport Correction

\begin{tabular}{|c|c|c|c|}
\hline \multirow{4}{*}{$\begin{array}{c}\text { IHECB/CSEWG } \\
\text { Benchmarks }\end{array}$} & \multicolumn{3}{|c|}{$k_{\text {eff }}$} \\
\hline & \multirow[b]{3}{*}{ menp } & \multicolumn{2}{|c|}{ COMBINE7.1 } \\
\hline & & Bondarenko & Bondarenko + Nordheim \\
\hline & & Self shielding & self shielding \\
\hline GODIVA (hmf-001) & $1.00014(22)$ & 1.00273 & 1.00273 \\
\hline FLATTOP-25 (hmf-028) & $1.00275(24)$ & 1.00654 & 1.00633 \\
\hline FLATTOP-Pu (pmf-006) & $1.00067(25)$ & 1.00492 & 1.00486 \\
\hline JEZEBEL (pmf-001 bare) & $1.0001(20)$ & 1.00274 & 1.00274 \\
\hline JEZEBEL-23 (umf-001) & $1.00032(21)$ & 1.00278 & 1.00278 \\
\hline JEZEBEL-Pu (pmf-002) & $1.00025(20)$ & 1.00336 & 1.00336 \\
\hline umf002-1 & $0.99891(21)$ & $1.00337 / 1.00294$ & $1.00337 / 1.00294$ \\
\hline umf002-2 & $1.00002(21)$ & $1.00527 / 1.00469$ & $1.00527 / 1.00419$ \\
\hline umf005-1 & $0.99396(21)$ & $1.00136 / 0.99749$ & $1.00089 / 0.99748$ \\
\hline umf005-2 & $0.99201(23)$ & $1.00089 / 0.99686$ & $1.00089 / 0.99686$ \\
\hline pci-001 & $1.01150(28)$ & 1.00991 & 1.00497 \\
\hline hci-004 & $0.98781(24)$ & 0.99223 & 0.98942 \\
\hline ORNL-1 (hst-013 Case 1) & & 0.99890 & 0.99881 \\
\hline ORNL-2 (hst-013 Case 2) & & 0.99802 & 0.99787 \\
\hline ORNL-10 (hst-032) & & 0.99925 & 0.99926 \\
\hline L-7 (hst009-3) & & 1.00577 & 1.00485 \\
\hline L-8 & & 1.00639 & 1.00632 \\
\hline L-9 & & 1.00380 & 1.00367 \\
\hline $\mathrm{L}-10$ & & 1.00298 & 1.00247 \\
\hline L-11 (hst012) & & 1.00183 & 1.00175 \\
\hline PNL-1 & & 1.01074 & 1.01031 \\
\hline PNL-2 & & 1.00741 & 1.00687 \\
\hline PNL-3 & & 0.99623 & 0.99596 \\
\hline PNL-4 & & 1.00202 & 1.00169 \\
\hline TRX1-r ${ }^{*}$ & $0.99908(29)$ & 1.00254 & 0.99890 \\
\hline TRX2-r ${ }^{* *}$ & $0.99908(28)$ & 1.00501 & 1.00316 \\
\hline BAPL1-r ${ }^{* *}$ & & 1.00465 & 1.00395 \\
\hline BAPL2-r ${ }^{* *}$ & & 1.00167 & 1.00169 \\
\hline BAPL3-r ${ }^{* *}$ & & 1.00286 & 1.00301 \\
\hline
\end{tabular}

Note: /1-D transport correction 
Table D.5: Calculated keff eigenvalues against the measured keff $=1.000 \mathrm{~B} 3$ and Sn Transport Correction

\begin{tabular}{|c|c|c|c|}
\hline \multirow[b]{3}{*}{$\begin{array}{c}\text { IHECB/CSEWG } \\
\text { Benchmarks }\end{array}$} & \multicolumn{3}{|c|}{$\boldsymbol{k}_{\text {eff }}$} \\
\hline & & \multicolumn{2}{|c|}{ COMBINE7.1 } \\
\hline & menp & $\begin{array}{c}\text { Bondarenko } \\
\text { Self shielding }\end{array}$ & $\begin{array}{c}\text { Bondarenko + } \\
\text { Nordheim } \\
\text { self shielding } \\
\end{array}$ \\
\hline GODIVA (hmf-001) & $1.00014(22)$ & 1.00268 & 1.00266 \\
\hline FLATTOP-25 (hmf-028) & $1.00275(24)$ & 1.00653 & 1.00632 \\
\hline FLATTOP-Pu (pmf-006) & $1.00067(25)$ & 1.00491 & 1.00484 \\
\hline JEZEBEL (pmf-001 bare) & $1.0001(20)$ & 1.00268 & 1.00267 \\
\hline JEZEBEL-23 (umf-001) & $1.00032(21)$ & 1.00261 & 1.00259 \\
\hline JEZEBEL-Pu (pmf-002) & $1.00025(20)$ & 1.00334 & 1.00333 \\
\hline ORNL-1 (hst-013 Case 1) & $0.99891(21)$ & 0.99768 & 0.99758 \\
\hline umf002-1 & $1.00002(21)$ & $1.00325 / 1.00282$ & $1.00323 / 1.00288$ \\
\hline umf002-2 & $0.99396(21)$ & $1.00517 / 1.00459$ & $1.00514 / 1.00456$ \\
\hline umf005-1 & $0.99201(23)$ & 0.99999/0.99574 & $0.99954 / 0.99572$ \\
\hline umf005-2 & $1.01150(28)$ & $0.99655 / 0.99469$ & $0.99654 / 0.99469$ \\
\hline pci-001 & $0.98781(24)$ & 1.00991 & 1.00497 \\
\hline hci-004 & & 0.99223 & 0.98936 \\
\hline ORNL-2 (hst-013 Case 2) & & 0.99679 & 0.99666 \\
\hline ORNL-10 (hst-032) & & 0.99867 & 0.99868 \\
\hline L-7 (hst009-3) & & 1.00446 & 1.00355 \\
\hline L-8 & & 1.00487 & 1.00480 \\
\hline L-9 & & 1.00260 & 1.00250 \\
\hline L-10 & & 1.00167 & 1.00116 \\
\hline L-11 (hst012) & & 1.00073 & 1.00064 \\
\hline PNL-1 & & 1.00848 & 1.00806 \\
\hline PNL-2 & & 1.00428 & 1.00373 \\
\hline PNL-3 & & 0.99429 & 0.99401 \\
\hline PNL-4 & & 0.99975 & 0.99943 \\
\hline TRX1-r & $0.99908(29)$ & 1.00162 & 0.99807 \\
\hline TRX2-r** & $0.99908(28)$ & 1.00432 & 1.00245 \\
\hline BAPL1-r ${ }^{* *}$ & & 1.00387 & 1.00318 \\
\hline BAPL2-r ${ }^{* *}$ & & 1.00095 & 1.00096 \\
\hline BAPL3-r ${ }^{* *}$ & & 1.00227 & 1.00243 \\
\hline
\end{tabular}

Note: /1-D transport correction 


\section{COMBINE7 EVALUATION OF THE BENCHMARK FOR THE DOPPLER REACTIVITY DEFECT}

A set of computational benchmarks for the Doppler reactivity defect has been specified for fuel pin cells containing normal or enriched $\mathrm{UO}_{2}$ fuel, reactor-recycle mixed-oxide (MOX) fuel, or weapons-grad MOX fuel. This Doppler benchmark has been approved by the Joint Benchmark Committee of the Mathematics and Computations, Reactor Physics, and Radiation Protection and Shielding Division of the American Nuclear Society. Reference (4) describes the benchmark specifications and provides a summary of the results submitted for it from many nuclear organizations. These benchmarks simulate the Doppler defect between hot zero power (HZP) at hot full power (HFP) for 16 different types of fuels in a PWR lattice. The temperature of the fuel is assumed to rise from $600 \mathrm{~K}$ to $900 \mathrm{~K}$. It is supposed that radius fuel pin depends on fuel temperature. The moderator is borated water and contains 1400 PPM of boron. The clad is supposed to be pure natural zirconium.

The reference pin cell is that described in Fig D.1 where, from the center to the exterior, the regions are: the fuel (with external radius of $0.39398 \mathrm{~cm}$ and $0.39433 \mathrm{~cm}$ respectively for HZP and HFP), the gap (with external radius of $0.40226 \mathrm{~cm}$ ), the clad (with external radius of $0.45972 \mathrm{~cm}$ ) and finally the coolant. The total width of the cell is $1.26678 \mathrm{~cm}$. The geometry for the benchmark is an infinite array of identical, infinitely long fuel pin cells with no axial variation. The isotopic concentrations for the benchmarks are provided in Tables D-6 through D-11. All these atomic numbers are from Reference 5.

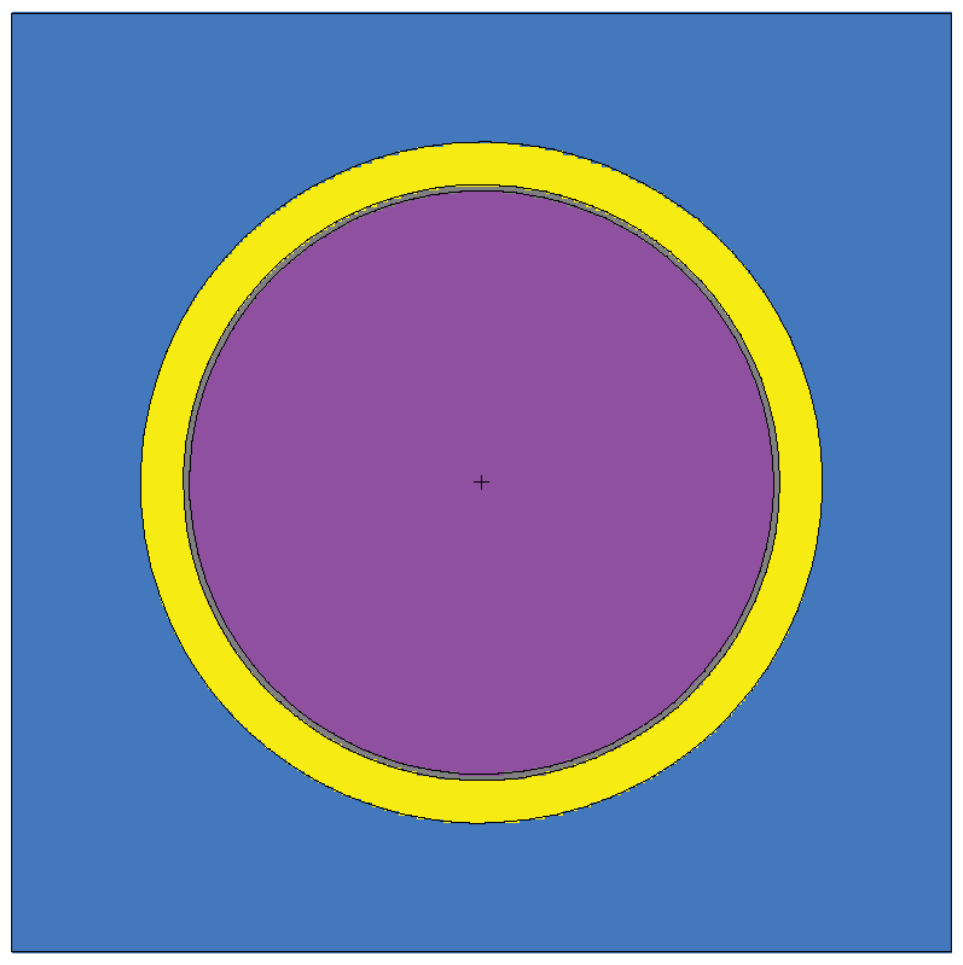

Figure D.1 Schematic of the Geometry for the Benchmarks

Table D-6, $\mathrm{UO}_{2}$ Fuel Composition at $600 \mathrm{~K}$ (atoms/b-cm)

\begin{tabular}{|c|c|c|c|c|}
\hline $\begin{array}{c}\text { Enrichment } \\
(\text { wt.\%) }\end{array}$ & ${ }^{16} \mathrm{O}$ & ${ }^{234} \mathrm{U}$ & ${ }^{235} \mathrm{U}$ & ${ }^{238} \mathrm{U}$ \\
\hline
\end{tabular}




\begin{tabular}{|c|c|c|c|c|}
\hline 0.711 & $4.61171 \times 10^{-2}$ & 0 & $1.66029 \times 10^{-4}$ & $2.28925 \times 10^{-2}$ \\
\hline 1.6 & $4.61218 \times 10^{-2}$ & $3.00175 \times 10^{-6}$ & $3.73618 \times 10^{-4}$ & $2.26843 \times 10^{-2}$ \\
\hline 2.4 & $4.61260 \times 10^{-2}$ & $4.50257 \times 10^{-6}$ & $5.60420 \times 10^{-4}$ & $2.24981 \times 10^{-2}$ \\
\hline 3.1 & $4.61297 \times 10^{-2}$ & $5.81576 \times 10^{-6}$ & $7.23867 \times 10^{-4}$ & $2.23352 \times 10^{-2}$ \\
\hline 3.9 & $4.61339 \times 10^{-2}$ & $7.31651 \times 10^{-6}$ & $9.10661 \times 10^{-4}$ & $2.21490 \times 10^{-2}$ \\
\hline 4.5 & $4.61371 \times 10^{-2}$ & $8.44205 \times 10^{-6}$ & $1.05075 \times 10^{-4}$ & $2.20093 \times 10^{-2}$ \\
\hline 5.0 & $4.61397 \times 10^{-2}$ & $9.37998 \times 10^{-6}$ & $1.16749 \times 10^{-4}$ & $2.18930 \times 10^{-2}$ \\
\hline
\end{tabular}

Table D-7, $\mathrm{UO}_{2}$ Fuel Composition at $900 \mathrm{~K}$ (atoms/b-cm)

\begin{tabular}{|c|c|c|c|c|}
\hline $\begin{array}{c}\text { Enrichment } \\
\text { (wt.\%) }\end{array}$ & ${ }^{16} \mathrm{O}$ & ${ }^{234} \mathrm{U}$ & ${ }^{235} \mathrm{U}$ & ${ }^{238} \mathrm{U}$ \\
\hline 0.711 & $4.59967 \times 10^{-2}$ & 0 & $1.65595 \times 10^{-4}$ & $2.28328 \times 10^{-2}$ \\
\hline 1.6 & $4.60014 \times 10^{-2}$ & $2.99391 \times 10^{-6}$ & $3.72462 \times 10^{-4}$ & $2.26251 \times 10^{-2}$ \\
\hline 2.4 & $4.60056 \times 10^{-2}$ & $4.40981 \times 10^{-6}$ & $5.58956 \times 10^{-4}$ & $2.24393 \times 10^{-2}$ \\
\hline 3.1 & $4.60093 \times 10^{-2}$ & $5.80057 \times 10^{-6}$ & $7.21977 \times 10^{-4}$ & $2.22768 \times 10^{-2}$ \\
\hline 3.9 & $4.60134 \times 10^{-2}$ & $7.29740 \times 10^{-6}$ & $9.08283 \times 10^{-4}$ & $2.20911 \times 10^{-2}$ \\
\hline 4.5 & $4.60166 \times 10^{-2}$ & $8.42000 \times 10^{-6}$ & $1.04801 \times 10^{-4}$ & $2.19519 \times 10^{-2}$ \\
\hline 5.0 & $4.60192 \times 10^{-2}$ & $9.35548 \times 10^{-6}$ & $1.16445 \times 10^{-4}$ & $2.18358 \times 10^{-2}$ \\
\hline
\end{tabular}

Table D-8, Reactor-Recycle MOX Fuel Composition at $600 \mathrm{~K}$ (atoms/b-cm)

\begin{tabular}{|c|c|c|c|}
\hline $\begin{array}{l}\mathrm{MOX}^{(} \mathrm{PuO}_{2} \text { wt. \%) } \\
\mathrm{Pu}^{2}\end{array}$ & ${ }^{16} \mathrm{O}$ & ${ }^{235} \mathrm{U}$ & ${ }^{238} \mathrm{U}$ \\
\hline 1.0 & $4.61140 \times 10^{-2}$ & $1.64368 \times 10^{-4}$ & $2.26636 \times 10^{-2}$ \\
\hline 2.0 & $4.61108 \times 10^{-2}$ & $1.62708 \times 10^{-4}$ & $2.24347 \times 10^{-2}$ \\
\hline 4.0 & $4.61042 \times 10^{-2}$ & $1.59387 \times 10^{-4}$ & $2.19768 \times 10^{-2}$ \\
\hline 6.0 & $4.60977 \times 10^{-2}$ & $1.56067 \times 10^{-4}$ & $2.15190 \times 10^{-2}$ \\
\hline 8.0 & $4.60912 \times 10^{-2}$ & $1.52746 \times 10^{-4}$ & $2.10611 \times 10^{-2}$ \\
\hline
\end{tabular}

\begin{tabular}{|c|c|c|c|c|}
\hline $\begin{array}{l}\text { MOX Composition } \\
\left(\mathrm{PuO}_{2} \text { wt. \%) }\right.\end{array}$ & ${ }^{239} \mathrm{Pu}$ & ${ }^{240} \mathrm{Pu}$ & ${ }^{241} \mathrm{Pu}$ & ${ }^{242} \mathrm{Pu}$ \\
\hline 1.0 & $1.03031 \times 10^{-4}$ & $6.86872 \times 10^{-5}$ & $3.43436 \times 10^{-5}$ & $2.28957 \times 10^{-5}$ \\
\hline 2.0 & $2.06062 \times 10^{-4}$ & $1.37374 \times 10^{-4}$ & $6.86872 \times 10^{-5}$ & $4.57915 \times 10^{-5}$ \\
\hline 4.0 & $4.12123 \times 10^{-4}$ & $2.72749 \times 10^{-4}$ & $1.37374 \times 10^{-4}$ & $9.15830 \times 10^{-5}$ \\
\hline 6.0 & $6.18185 \times 10^{-4}$ & $4.12123 \times 10^{-4}$ & $2.06062 \times 10^{-4}$ & $1.37374 \times 10^{-4}$ \\
\hline 8.0 & $8.24247 \times 10^{-4}$ & $5.49498 \times 10^{-4}$ & $2.74749 \times 10^{-4}$ & $1.83166 \times 10^{-4}$ \\
\hline
\end{tabular}

Table D-9, Reactor-Recycle MOX Fuel Composition at $900 \mathrm{~K}$ (atoms/b-cm)

\begin{tabular}{|c|c|c|c|}
\hline $\begin{array}{l}\text { MOX Composition } \\
\left(\mathrm{PuO}_{2} \text { wt. \% }\right)\end{array}$ & ${ }^{16} \mathrm{O}$ & ${ }^{235} \mathrm{U}$ & ${ }^{238} \mathrm{U}$ \\
\hline 1.0 & $4.59936 \times 10^{-2}$ & $1.63939 \times 10^{-4}$ & $2.26044 \times 10^{-2}$ \\
\hline 2.0 & $4.59904 \times 10^{-2}$ & $1.62283 \times 10^{-4}$ & $2.23761 \times 10^{-2}$ \\
\hline 4.0 & $4.59838 \times 10^{-2}$ & $1.58971 \times 10^{-4}$ & $2.19194 \times 10^{-2}$ \\
\hline 6.0 & $4.59773 \times 10^{-2}$ & $1.55659 \times 10^{-4}$ & $2.14628 \times 10^{-2}$ \\
\hline 8.0 & $4.59708 \times 10^{-2}$ & $1.52347 \times 10^{-4}$ & $2.10061 \times 10^{-2}$ \\
\hline
\end{tabular}

\begin{tabular}{|c|c|c|c|c|}
\hline $\begin{array}{l}\text { MOX Composition } \\
\left(\mathrm{PuO}_{2} \text { wt. \%) }\right.\end{array}$ & ${ }^{239} \mathrm{Pu}$ & ${ }^{240} \mathrm{Pu}$ & ${ }^{241} \mathrm{Pu}$ & ${ }^{242} \mathrm{Pu}$ \\
\hline 1.0 & $1.02762 \times 10^{-4}$ & $6.85079 \times 10^{-5}$ & $3.42539 \times 10^{-5}$ & $2.28360 \times 10^{-5}$ \\
\hline 2.0 & $2.05524 \times 10^{-4}$ & $1.37016 \times 10^{-4}$ & $6.85079 \times 10^{-5}$ & $4.56719 \times 10^{-5}$ \\
\hline 4.0 & $4.11047 \times 10^{-4}$ & $2.74031 \times 10^{-4}$ & $1.37016 \times 10^{-4}$ & $9.13438 \times 10^{-5}$ \\
\hline 6.0 & $6.16751 \times 10^{-4}$ & $4.11047 \times 10^{-4}$ & $2.05524 \times 10^{-4}$ & $1.37016 \times 10^{-4}$ \\
\hline
\end{tabular}


Table D-10, Weapons-Grade MOX Fuel Composition at $600 \mathrm{~K}$ (atoms/b-cm)

\begin{tabular}{|c|c|c|c|}
\hline $\begin{array}{l}\mathrm{MOX} \text { Composition } \\
\left(\mathrm{PuO}_{2} \text { wt. \% }\right)\end{array}$ & ${ }^{16} \mathrm{O}$ & ${ }^{235} \mathrm{U}$ & ${ }^{238} \mathrm{U}$ \\
\hline 1.0 & $4.61154 \times 10^{-2}$ & $1.64368 \times 10^{-4}$ & $2.26636 \times 10^{-2}$ \\
\hline 2.0 & $4.61136 \times 10^{-2}$ & $1.62708 \times 10^{-4}$ & $2.24347 \times 10^{-2}$ \\
\hline 4.0 & $4.61099 \times 10^{-2}$ & $1.59387 \times 10^{-4}$ & $2.19768 \times 10^{-2}$ \\
\hline 6.0 & $4.61061 \times 10^{-2}$ & $1.56067 \times 10^{-4}$ & $2.15190 \times 10^{-2}$ \\
\hline
\end{tabular}

\begin{tabular}{|c|c|c|c|c|}
\hline $\begin{array}{l}\text { MOX Composition } \\
\left(\mathrm{PuO}_{2} \text { wt. \%) }\right.\end{array}$ & ${ }^{239} \mathrm{Pu}$ & ${ }^{240} \mathrm{Pu}$ & ${ }^{241} \mathrm{Pu}$ & ${ }^{242} \mathrm{Pu}$ \\
\hline 1.0 & $2.14958 \times 10^{-4}$ & $1.35947 \times 10^{-5}$ & $9.18623 \times 10^{-5}$ & $2.29656 \times 10^{-7}$ \\
\hline 2.0 & $4.29916 \times 10^{-4}$ & $2.70994 \times 10^{-5}$ & $1.83725 \times 10^{-6}$ & $4.59312 \times 10^{-7}$ \\
\hline 4.0 & $8.59831 \times 10^{-4}$ & $5.41988 \times 10^{-5}$ & $3.67449 \times 10^{-6}$ & $9.18623 \times 10^{-7}$ \\
\hline 6.0 & $1.28975 \times 10^{-3}$ & $8.12982 \times 10^{-5}$ & $5.51174 \times 10^{-6}$ & $1.37794 \times 10^{-6}$ \\
\hline
\end{tabular}

Table D-11, Weapons-Grade MOX Fuel Composition at $900 \mathrm{~K}$ (atoms/b-cm)

\begin{tabular}{|c|c|c|c|}
\hline $\begin{array}{l}\left.\mathrm{MOX}^{(} \mathrm{PuO}_{2} \text { wt. } \%\right) \\
\text { \% })\end{array}$ & ${ }^{16} \mathrm{O}$ & ${ }^{235} \mathrm{U}$ & ${ }^{238} \mathrm{U}$ \\
\hline 1.0 & $4.59950 \times 10^{-2}$ & $1.63939 \times 10^{-4}$ & $2.26044 \times 10^{-2}$ \\
\hline 2.0 & $4.59932 \times 10^{-2}$ & $1.62283 \times 10^{-4}$ & $2.23761 \times 10^{-2}$ \\
\hline 4.0 & $4.59895 \times 10^{-2}$ & $1.58971 \times 10^{-4}$ & $2.19194 \times 10^{-2}$ \\
\hline 6.0 & $4.59857 \times 10^{-2}$ & $1.55659 \times 10^{-4}$ & $2.14628 \times 10^{-2}$ \\
\hline
\end{tabular}

\begin{tabular}{|c|c|c|c|c|}
\hline $\begin{array}{l}\text { MOX Composition } \\
\left(\mathrm{PuO}_{2} \text { wt. \%) }\right.\end{array}$ & ${ }^{239} \mathrm{Pu}$ & ${ }^{240} \mathrm{Pu}$ & ${ }^{241} \mathrm{Pu}$ & ${ }^{242} \mathrm{Pu}$ \\
\hline 1.0 & $2.14397 \times 10^{-4}$ & $1.35143 \times 10^{-5}$ & $9.16224 \times 10^{-7}$ & $2.29056 \times 10^{-7}$ \\
\hline 2.0 & $4.28793 \times 10^{-4}$ & $2.70286 \times 10^{-5}$ & $1.83245 \times 10^{-6}$ & $4.58112 \times 10^{-7}$ \\
\hline 4.0 & $8.57586 \times 10^{-4}$ & $5.40572 \times 10^{-5}$ & $3.66490 \times 10^{-6}$ & $9.16224 \times 10^{-7}$ \\
\hline 6.0 & $1.28638 \times 10^{-3}$ & $8.10859 \times 10^{-5}$ & $5.49735 \times 10^{-6}$ & $1.37434 \times 10^{-6}$ \\
\hline
\end{tabular}

To evaluate the COMBINE7.1 capabilities and limitations as a part of $\mathrm{V} \& \mathrm{~V}$, a series of calculations were performed, simulating these benchmarks. The results are compared to the in-house calculated $\mathrm{MCNP}^{3}$ results and the reported results in Reference 5.

The first series of calculations were for the HZP condition but having a uniform temperature at $300 \mathrm{~K}$. The ENDF/B-VII.0 derived cross sections ${ }^{4}$ were also used in MCNP runs. In the COMBINE/A runs, 18-group macroscopic cell cross sections were first generated and using these cross sections, a one-zone transport calculation was made. In the COMBINE/B runs, 18 macroscopic cross sections were first generated for the three zones (fuel, cladding, and moderator, respectively). Using these cross sections, a three-zone transport calculation was made. In the COMBINE/C runs, 167 macroscopic fine-group cross sections were first generated for the three zones. Using 
these cross sections, a three-zone transport calculation was made. These cross sections were spatially coalesced and then collapsed into 18 broad group cross sections. Using these cross sections, a one-zone transport calculation was made. In all transport calcuations, a white outer boundary condition with equivalent the cylindrical geometry (O.D. $1.4294 \mathrm{~cm}$ ) was used. The COMBINE results with the Bondareno self-shiding treatment are compared in Table D.12 through D-14 to those from the in-house MCNP calculations with the identical geometry, composition, and temperatures. The results with the additional Nordheim self-shielding treatment were almost identical to those from the Bodnrenko results. All the COMBINE eigenvaluse are favorably comparable with those from the MCNP's. Still, the results indicate some instability in the cross section coalescing.

Table D.12 Results for $\mathrm{UO}_{2}$ Pin cells

\begin{tabular}{|c|c|c|c|c|}
\hline \multirow{2}{*}{$\begin{array}{c}\text { Enrichment } \\
\text { (wt. \%) }\end{array}$} & \multicolumn{4}{|c|}{$k_{\text {eff }}$} \\
\cline { 2 - 5 } & MCNP & COMBINE/A & COMBINE/B & COMBINE/C \\
\hline 0.711 & 0.68227 & 0.68014 & 0.68232 & 0.68221 \\
\hline 1.6 & 0.97813 & 0.97705 & 0.97953 & 0.97918 \\
\hline 2.4 & 1.11498 & 1.11413 & 1.11713 & 1.11670 \\
\hline 3.1 & 1.19236 & 1.19097 & 1.19448 & 1.19397 \\
\hline 3.9 & 1.25440 & 1.25246 & 1.25649 & 1.25594 \\
\hline 4.5 & 1.28880 & 1.28706 & 1.29147 & 1.29096 \\
\hline 5.0 & 1.31347 & 1.31077 & 1.31551 & 1.31498 \\
\hline
\end{tabular}

Note: all the MCNP results are with \pm 0.00030

Table D.13 Results for Reactor-Recycle MOX Pin Cells

\begin{tabular}{|c|c|c|c|c|}
\hline \multirow{2}{*}{$\begin{array}{c}\text { MOX Composition } \\
\text { (PuO2 wt. \%) }\end{array}$} & \multicolumn{4}{|c|}{$k_{\text {eff }}$} \\
\cline { 2 - 5 } & MCNP & COMBINE/A & COMBINE/B & COMBINE/C \\
\hline 1.0 & 0.93459 & 0.93360 & 0.93464 & 0.93548 \\
\hline 2.0 & 1.02048 & 1.02052 & 1.02108 & 1.02300 \\
\hline 4.0 & 1.08587 & 1.08718 & 1.08720 & 1.09023 \\
\hline 6.0 & 1.11844 & 1.12031 & 1.11980 & 1.12336 \\
\hline 8.0 & 1.14315 & 1.14589 & 1.14503 & 1.14865 \\
\hline
\end{tabular}

Table D.14 Results for Weapons-Grade MOX Pin Cells (w/ free)

\begin{tabular}{|c|c|c|c|c|}
\hline \multirow{2}{*}{$\begin{array}{c}\text { MOX Composition } \\
\text { (PuO2 wt. \%) }\end{array}$} & \multicolumn{4}{|c|}{$k_{\text {eff }}$} \\
\cline { 2 - 5 } & MCNP & COMBINE/A & COMBINE/B & COMBINE/C \\
\hline 1.0 & 1.08538 & 1.08361 & 1.08587 & 1.08553 \\
\hline 2.0 & 1.19110 & 1.19078 & 1.19192 & 1.19142 \\
\hline 4.0 & 1.26885 & 1.27059 & 1.27002 & 1.26963 \\
\hline 6.0 & 1.30832 & 1.31005 & 1.30852 & 1.30840 \\
\hline
\end{tabular}

The rest of calculations were devoted to the HZP and HFP conditions, smulating the benchmarks with the COMBINE/A option and COMBINE/B option as previously described. Tables D.15 through D20 list the COMBINE results. The Doppler coefficient $(\mathrm{pcm} / \mathrm{K})$ of reativity as listed is determined as

$$
D C=\frac{\Delta \rho_{\text {Dop }}}{\Delta T_{\text {fuel }}}
$$

Where DC is the Doppler coefficient of reactivity, $\Delta \mathrm{T}_{\text {fuel }}$ is $300 \mathrm{~K}$, and the Doppler defect is

$$
\Delta \rho_{D O P}=\frac{k_{H F P}-k_{H Z P}}{k_{H F P} * k_{H Z P}} * 10,000
$$


Table D.15 Results for $\mathrm{UO}_{2}$ Pin cells (w/o free), COMBINE/A

\begin{tabular}{|c|c|c|c|}
\hline \multirow{2}{*}{$\begin{array}{l}\text { Enrichment } \\
(\text { wt. \%) }\end{array}$} & \multicolumn{2}{|c|}{$k_{\text {eff }}$} & \multirow{2}{*}{$\begin{array}{c}\text { Doppler Coefficient } \\
(\mathrm{pcm} / \mathrm{K})\end{array}$} \\
\cline { 2 - 3 } & HZP & HFP & -4.69 \\
\hline 0.711 & 0.66255 & 0.65644 & -3.24 \\
\hline 1.6 & 0.95841 & 0.94958 & -2.78 \\
\hline 2.4 & 1.09627 & 1.08633 & -2.57 \\
\hline 3.1 & 1.17375 & 1.16323 & -2.43 \\
\hline 3.9 & 1.23576 & 1.22473 & -2.34 \\
\hline 4.5 & 1.27065 & 1.25943 & -2.29 \\
\hline 5.0 & 1.29449 & 1.28310 & \\
\hline
\end{tabular}

Table D.16 Results for Reactor-Recycle MOX Pin Cells, COMBINE/A

\begin{tabular}{|c|c|c|c|}
\hline \multirow{2}{*}{$\begin{array}{c}\text { Enrichment } \\
(\text { wt. \%) }\end{array}$} & HZP & HFP & $\begin{array}{c}\text { Doppler Coefficient } \\
\text { (pcm/K) }\end{array}$ \\
\cline { 2 - 3 } & 0.94161 & 0.93092 & -4.06 \\
\hline 1.0 & 1.01817 & 1.00616 & -3.91 \\
\hline 2.0 & 1.07408 & 1.06141 & -3.70 \\
\hline 6.0 & 1.10321 & 1.09050 & -3.52 \\
\hline 8.0 & 1.12708 & 1.11430 & -3.39 \\
\hline
\end{tabular}

Table D.17 Results for Weapons-Grade MOX Pin Cells, COMBINE/A

\begin{tabular}{|c|c|c|c|}
\hline \multirow{2}{*}{$\begin{array}{l}\text { Enrichment } \\
\text { (wt. \%) }\end{array}$} & \multicolumn{2}{|c|}{$k_{\mathrm{eff}}$} & \multirow{2}{*}{$\begin{array}{c}\text { Doppler Coefficient } \\
(\mathrm{pcm} / \mathrm{K})\end{array}$} \\
\hline & $\mathrm{HZP}$ & $\mathrm{HFP}$ & \\
\hline 1.0 & 1.08620 & 1.07584 & -2.96 \\
\hline 2.0 & 1.17792 & 1.16560 & -2.99 \\
\hline 4.0 & 1.24799 & 1.23442 & -2.94 \\
\hline 6.0 & 1.28541 & 1.27229 & -2.67 \\
\hline
\end{tabular}


Table D.18 Results for UO2 Pin cells, COMBINE/B

\begin{tabular}{|c|c|c|c|}
\hline \multirow{2}{*}{$\begin{array}{c}\text { Enrichment } \\
(\text { wt. \%) }\end{array}$} & HZP & HFP & \multirow{2}{*}{$\begin{array}{c}\text { Doppler Coefficient } \\
(\mathrm{pcm} / \mathrm{K})\end{array}$} \\
\cline { 2 - 3 } & 0.66461 & 0.65785 & -5.15 \\
\hline 0.711 & 0.96072 & 0.95131 & -3.43 \\
\hline 1.6 & 1.09916 & 1.08870 & -2.91 \\
\hline 2.4 & 1.17711 & 1.16617 & -2.66 \\
\hline 3.1 & 1.23976 & 1.22837 & -2.49 \\
\hline 3.9 & 1.27512 & 1.26352 & -2.40 \\
\hline 4.5 & 1.29935 & 1.28763 & -2.34 \\
\hline 5.0 & & & \\
\hline
\end{tabular}

Table D.19 Results for Reactor-Recycle MOX Pin Cells, COMBINE/B

\begin{tabular}{|c|c|c|c|}
\hline \multirow{2}{*}{$\begin{array}{c}\text { Enrichment } \\
\text { (wt. \%) }\end{array}$} & HZP & HFP & \multirow{2}{*}{$\begin{array}{c}\text { Doppler Coefficient } \\
\text { (pcm/K) }\end{array}$} \\
\cline { 2 - 3 } & 0.94202 & 0.93070 & -4.31 \\
\hline 1.0 & 1.01806 & 1.00531 & -4.15 \\
\hline 2.0 & 1.07364 & 1.06037 & -3.89 \\
\hline 4.0 & 1.10253 & 1.08932 & -3.67 \\
\hline 6.0 & 1.12624 & 1.11318 & -3.47 \\
\hline
\end{tabular}

Table D.20 Results for Weapons-Grade MOX Pin Cells, COMBINE/B

\begin{tabular}{|c|c|c|c|}
\hline \multirow{2}{*}{$\begin{array}{c}\text { Enrichment } \\
(\text { wt. \%) }\end{array}$} & HZP & HFP & \multirow{2}{*}{$\begin{array}{c}\text { Doppler Coefficient } \\
(\mathrm{pcm} / \mathrm{K})\end{array}$} \\
\cline { 2 - 3 } & 1.08826 & 1.07750 & -3.06 \\
\hline 1.0 & 1.17883 & 1.16602 & -3.11 \\
\hline 2.0 & 1.24724 & 1.23322 & -3.04 \\
\hline 4.0 & 1.28384 & 1.26966 & -2.90 \\
\hline
\end{tabular}

All the eigenvalues are favorable to the reported results. All the Doppler coefficients are within $1 \sigma$ of the reported average values in Reference 5 but mostly in the lower side, i.e., more negative. It is also observed that the Doppler coefficnients obtained with a three-zone model are more negative than those obtained with the 0-D cell/one-zone transport calculations. 


\section{REFERENCE}

1. International Handbook of Evaluated Criticality Safety Benchmark Experiments, OECD Nuclear Energy Agency report NEA/NSC/DOC(95)03, September 2005 Edition.

2. Cross Section Evaluation Working Group Benchmark Specifications, ENDF-202, BNL-19302, November, 1974 and September 1991 Update.

3. MCNP2.6.0 Extensions, LA-UR-08-2216, April, 2008.

4. The ENDF/B-V.II.0-ACE Library, D0022MNYCP00, RSICC, Oak Ridge National, Labarotory, 2008.

5. Russell D Mosteller, The Doppler-defect benchmark: overview and summary of results, Joint International Topical Meeting on Mathematics \& Computation and Supercomputing in Nuclear Applications (M\&C + SNA 2007), Monterery, California, April,2007. 